\title{
THE LOCALISATION, INTRACELLULAR TRANSPORT, AND BIOSYNTHETIC REGULATION OF BETALAIN PLANT PIGMENTS
}

BY

KATE EMMA CALCOTT

A thesis

submitted to the Victoria University of Wellington in fulfilment of the requirements for the degree of

Doctor of Philosophy

Victoria University of Wellington

(2014) 


\section{Abstract}

This thesis investigates the localisation, transport and biosynthetic control of betalain plant pigments to compare with the extensively researched anthocyanins. Anthocyanins and betalains appear similar, yet no plant naturally contains both pigment types. Due to this mutual exclusivity, betalain pigments are thought to functionally replace anthocyanins in many Caryophyllales. However, minimal research has been conducted to support this replacement hypothesis, resulting in limited knowledge of betalain pigment distribution and biosynthesis. The following series of experiments have added to this body of knowledge.

Localisation of betalains was compared with that reported for anthocyanins. Histological analyses of 12 different betalain-producing species revealed similar pigment localisation to that of anthocyanic species. Similarities in pigment localisation suggest that these pigment types may have similar functional roles. The histological analyses also found that betacyanins and betaxanthins had differential localisation in several taxa. Organ- or tissue-specific distribution of betalain compounds suggests differing biological functions for betaxanthins and betacyanins.

Hypotheses on betalain transport were tested using transgenic Arabidopsis thaliana lines capable of producing anthocyanins (PAP1-5), betalains (DOD-6), or both (DOD-6 x PAP1-5). Betaxanthins appeared to use vesicular transport, as betaxanthins were detected in small circular bodies within the cytoplasm. Furthermore, this observation suggests that betaxanthin formation occurred outside of the vacuole. DOD-6 was also crossed with Arabidopsis mutants, $t t 12$ and $t t 19$, which are deficient in proteins required for flavonoid vacuolar transport. Betaxanthin accumulation was reduced in both lines. In addition, DOD-6 was treated with transport inhibitors that affect anthocyanin accumulation. These experiments demonstrated that betaxanthins can utilise known flavonoid transport mechanisms, at least in this artificial pigment system.

Regulation of betalain biosynthesis was analysed using Swiss chard (Beta vulgaris subsp. cicla $\mathrm{cv}$. 'Bright Lights'). Betalain production was induced through physical wounding of the lamina in red and white Swiss chard lines. Betalain pigments were produced around the wounding sites in the red line but not in the white line. Transcript level changes of betalain and flavonoid biosynthetic genes in these tissues were 
measured using real-time quantitative polymerase chain reaction analyses. Betalain biosynthetic genes were not up-regulated in the red line even though red pigments visibly accumulated. Rather, these genes were already expressed in the red line prior to wounding. Biosynthetic control of betalains may either be earlier in the pathway or at the post-transcriptional level. In contrast, all three flavonoid biosynthetic genes were up-regulated in response to wounding, indicating that expression of flavonoid and betalain biosynthetic genes are not co-regulated in Swiss chard.

The final set of experiments examined the function of the two Beta vulgaris DOD genes (DODA and DODA1). Both genes were transiently expressed in nivea Antirrhinum majus dorsal petals and vacuum infiltrated with the betalain precursor L-DOPA. Expression of $D O D A 1$ but not $D O D A$ appeared to produce betalains. DODA-like genes have been found in anthocyanin-producing species, suggesting that this gene may not be involved in betalain biosynthesis.

The overall findings from this research indicate that betalain pigment evolution of may have involved the utilization of anthocyanin transport machinery, but the regulatory control of the two pathways appears different. 


\section{Acknowledgements}

I wish to begin by thanking my supervisors, Dr Kathy Schwinn, Dr Kevin Davies and Professor Kevin Gould, for all their support and encouragement during my $\mathrm{PhD}$ research. I would particularly like to thank Kathy and Kevin D. for taking me under their wings when my research went a bit wayward. You helped guide me back on course whilst making me feel like family. For that, I will be forever grateful.

Thank you to Hanh Ngo, Lei Wang, Dr Don Hunter, Dr David Lewis, Dr Simon Deroles, Dr Kathy Schwinn and Dr Murray Boase for teaching me a wide range of molecular biology techniques. For the most amazing technical assistance, I wish to thank Steve Arathoon, Ian King, Julie Ryan and Andrew Mullan. I thank Dr Jianyu Chen for helping me with confocal microscopy and Tony Corbett for assisting in poster creation. Thank you to Nigel Joyce for running LC-MS analyses, Dr Lesley Larsen for supplying me with cyclo-DOPA, and Dr Andy Allan for the use of the Arabidopsis thaliana PAP1 over-expression lines.

During my research I was able to get to know some of the most incredible people. To the Plant Pigments team - thank you for being my Palmerston North family. Thank you to my rock Dr Jovyn $\mathrm{Ng}$, for making the final year of my $\mathrm{PhD}$ one of the most enjoyable of my life. Thank you to Patricia Stein for helping me to overcome numerous rough patches. And to my lab-in-laws, you guys are awesome (especially Becky).

To all of my family and friends, thank you for your unwavering support.

I thank the following for financial assistance during my $\mathrm{PhD}$ : Victoria University of Wellington, Universities NZ, the Royal Society of New Zealand, Wellington Botanical Society, New Zealand Society of Plant Biologists, and the Todd Foundation. A special thanks to Plant \& Food Research for hosting me during this time.

Lastly, I want to thank my husband. You allowed me to dream big, and aim even higher. 


\section{Table of contents}

Abstract.........................................................................................................................ii

Acknowledgements.......................................................................................................................... v

Table of Contents .......................................................................................................vi

List of Figures.................................................................................................................

List of Tables .............................................................................................................. $\mathrm{xv}$

Abbreviations ........................................................................................................................ $\mathrm{xv}$

Chapter 1: General introduction ............................................................................................1

$1.1 \quad$ Thesis background ...................................................................................

1.2 Plant pigments: betalains and anthocyanins ................................................

$1.3 \quad$ Evolution and mutual exclusivity .................................................................

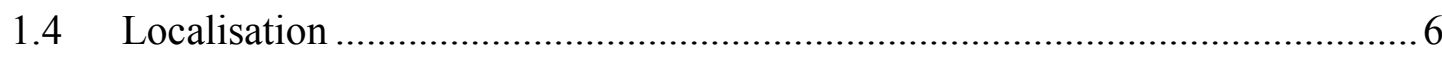

1.5 Betalain and anthocyanin production is induced by similar factors................... 8

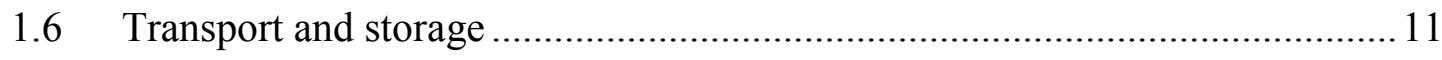

1.7 Biosynthetic pathways................................................................................... 15

1.7.1 Flavonoid biosynthesis............................................................................ 15

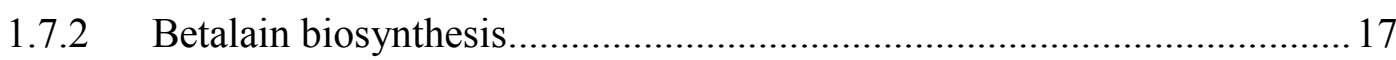

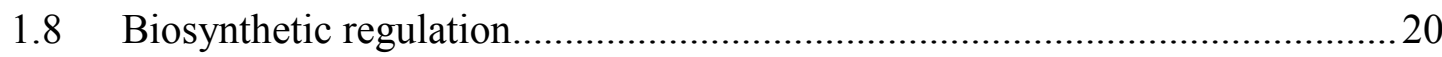

1.9 Pigmentation mutants in pigment biosynthesis research...................................2

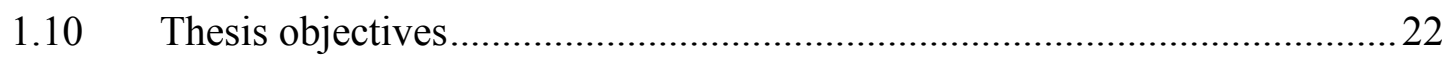

Chapter 2: General methods ......................................................................................................23

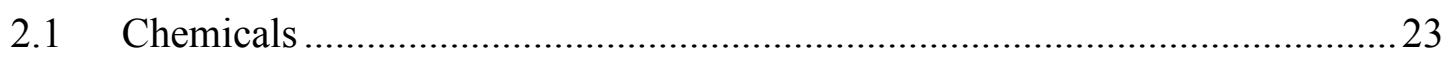

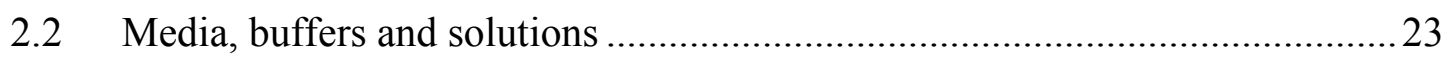

2.3 Plant growth conditions and treatments ........................................................... 23

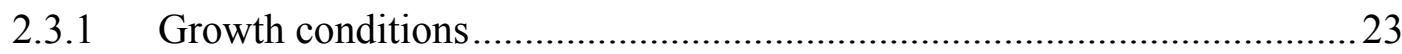

2.3.2 Vegetative tissue and seed surface sterilisation ........................................23

2.4 General microscopy details ...................................................................... 24 


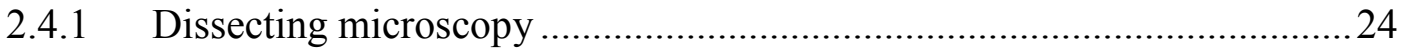

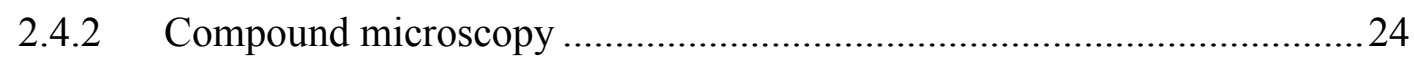

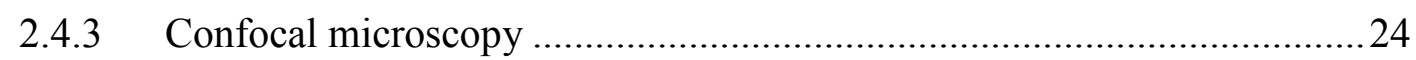

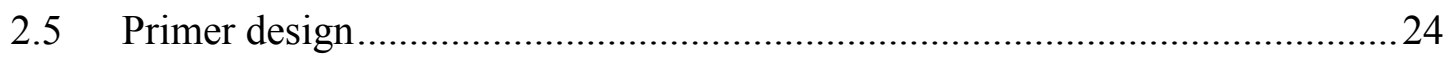

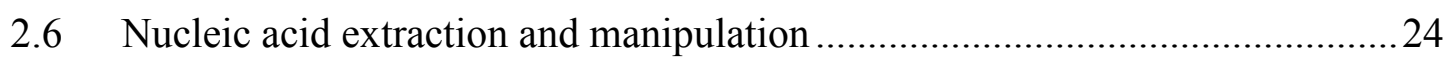

2.6.1 Genomic DNA extraction ................................................................. 24

2.6.2 RNA extraction and complementary DNA (cDNA) synthesis .................25

2.6.3 Assessment of DNA and RNA quality and quantity............................25

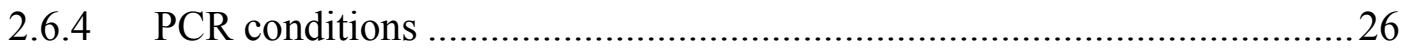

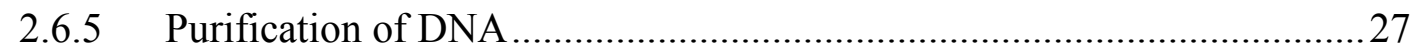

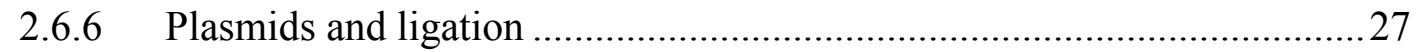

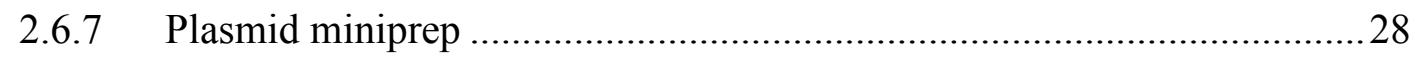

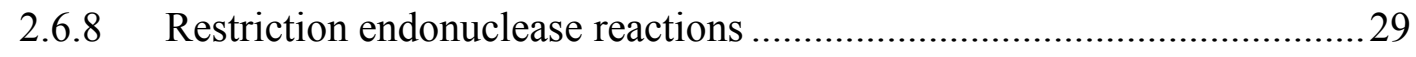

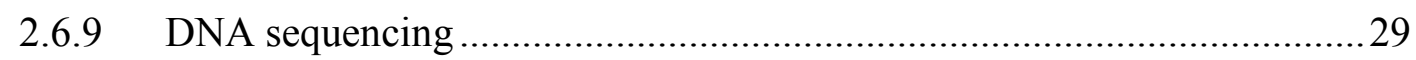

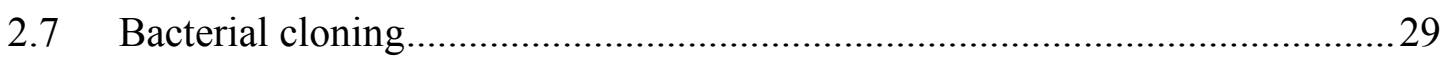

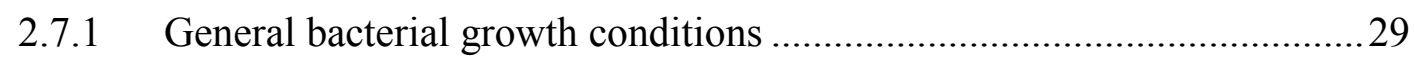

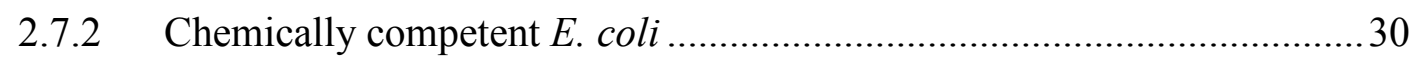

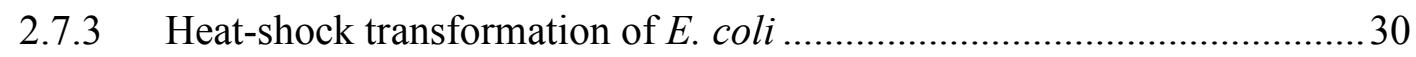

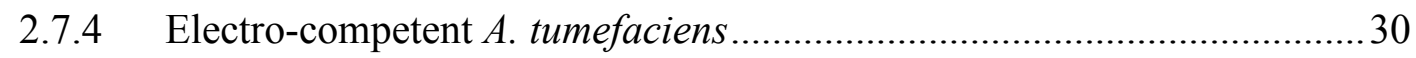

2.7.5 Electroporation transformation of $A$. tumefaciens .................................. 30

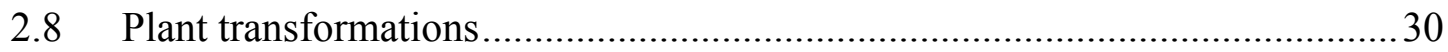

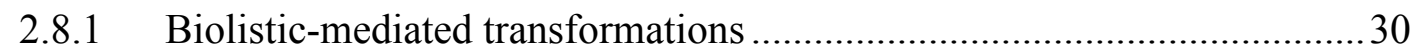

2.8.2 Agrobacterium-mediated transformations .......................................... 31

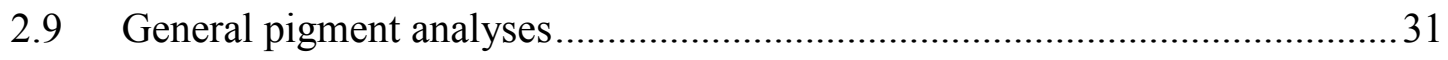

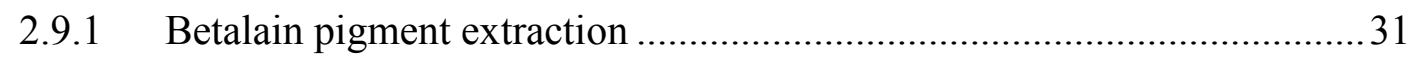

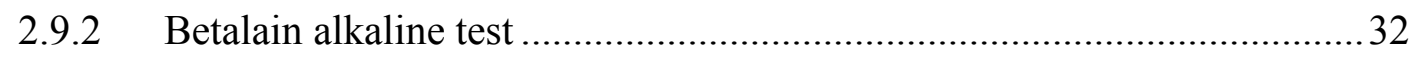

Chapter 3: Betalain localisation ..................................................................................................33 


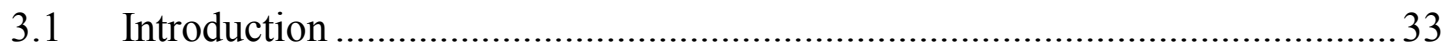

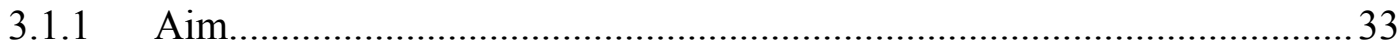

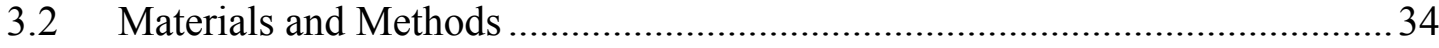

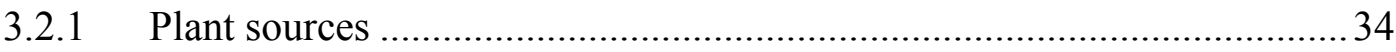

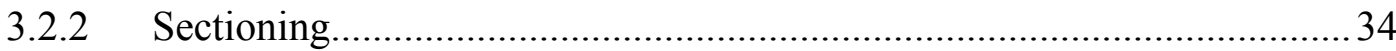

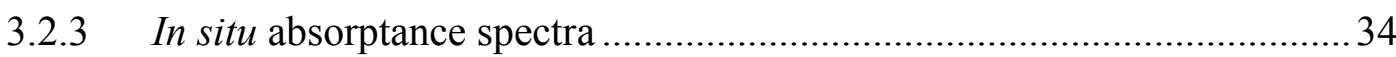

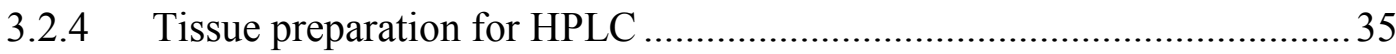

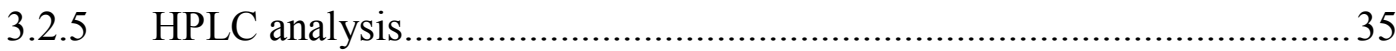

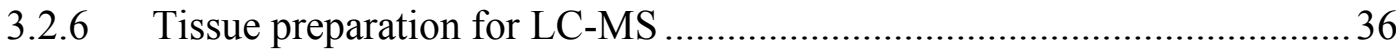

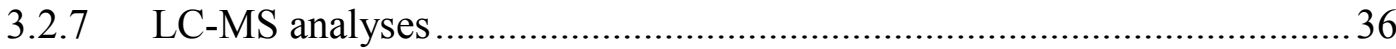

3.2.8 Photometric quantification and identification of betalains ......................37

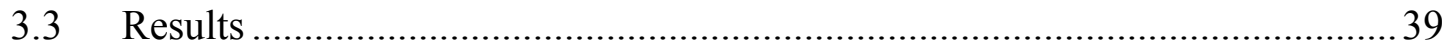

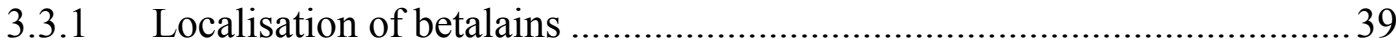

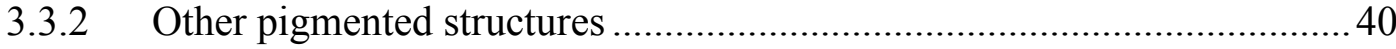

3.3.3 Differential localisation of betaxanthins and betacyanins ....................... 40

3.3.4 Pigment analyses of Swiss chard ..................................................... 41

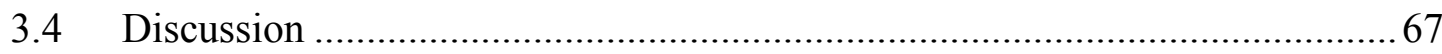

3.4.1 Developmental origin of similar pigmented tissues...............................67

3.4.2 Betalain localisation is similar to anthocyanins .......................................67 67

3.4.3 Betalainic compound distribution is uneven within and between organs 69

3.4.4 Physiological roles of betalains in vegetative tissues .............................71

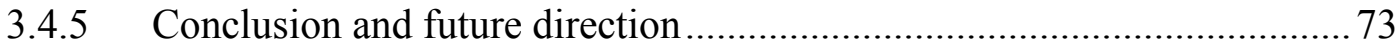

Chapter 4: Development of a betalain model system in Arabidopsis thaliana ...........75

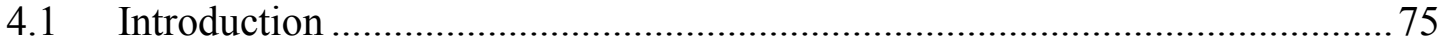

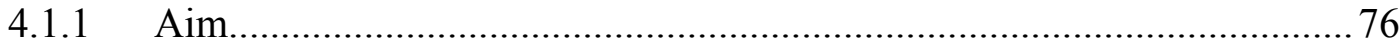

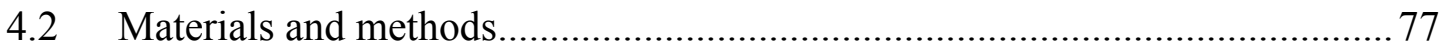




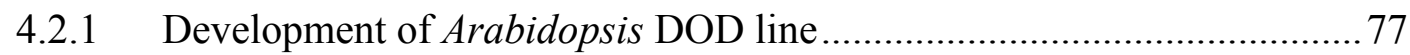

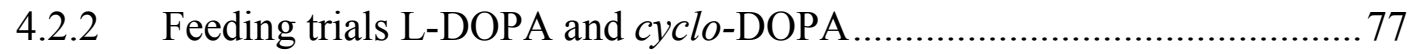

4.2.3 Creation of $D O D / C Y P 76 A D 1$ double transgenic .................................... 79

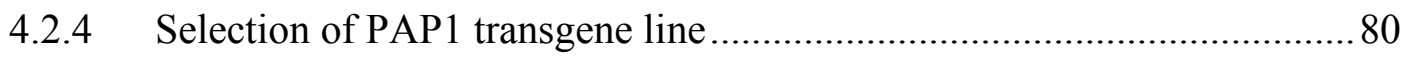

4.2.5 Arabidopsis crosses: DOD-6 with $t t 12$, $t$ t19 and PAP1-5....................... 80

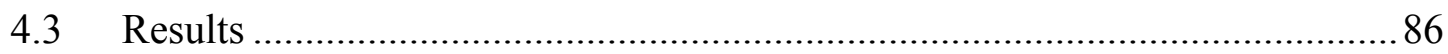

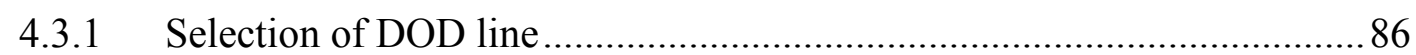

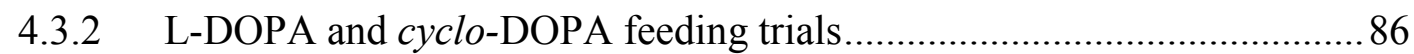

4.3.3 Creation of $D O D / C Y P 76 A D 1$ double transgenic plants ..........................8 88

4.3.4 Selection of PAP1-5 and DOD-6 x PAP1-5 plants.................................. 88

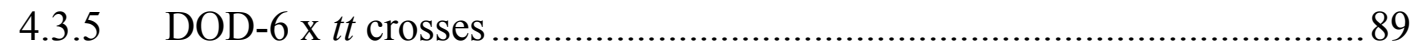

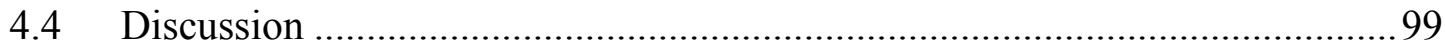

4.4.1 Physiological testing of betalains and anthocyanins ..............................99

4.4.2 Conclusions and future directions ................................................... 102

Chapter 5: Storage and transport...................................................................103

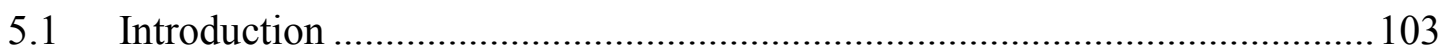

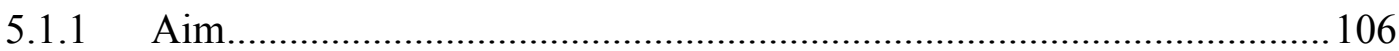

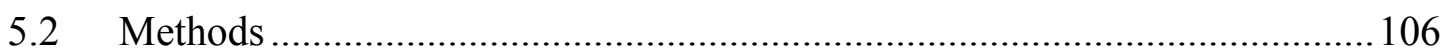

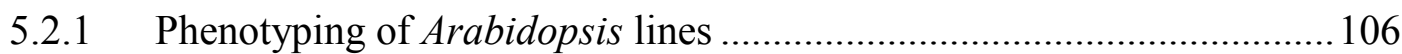

5.2.2 Anthocyanin and betalain induction in DOD-6 .................................. 106

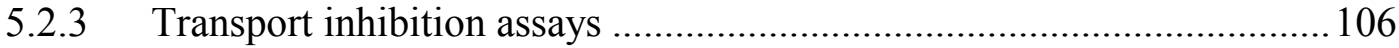

5.2.4 Anthocyanin and betalain extractions ................................................... 107

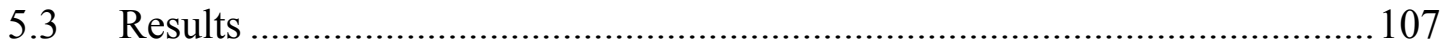

5.3.1 Betalains and anthocyanins can co-localise ....................................... 107

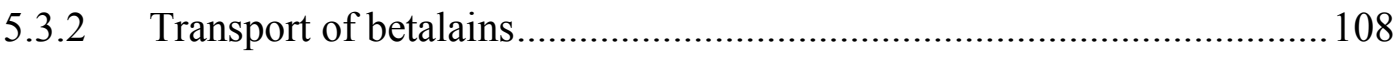

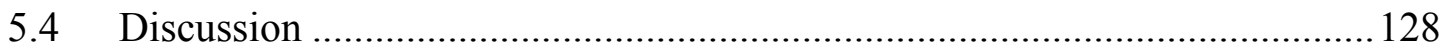

5.4.1 Co-localisation of betalains and anthocyanins ..................................... 128 


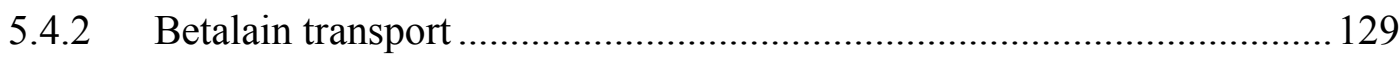

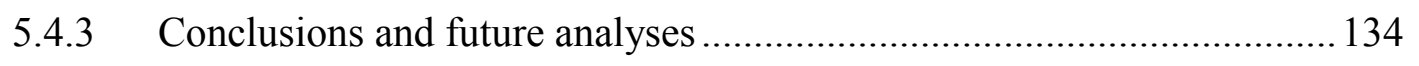

Chapter 6: Betalain biosynthetic control ...............................................................137

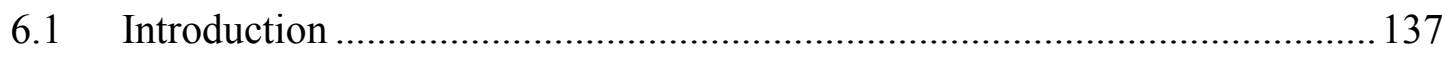

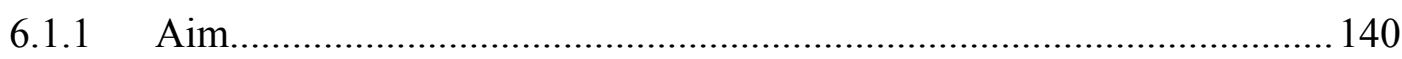

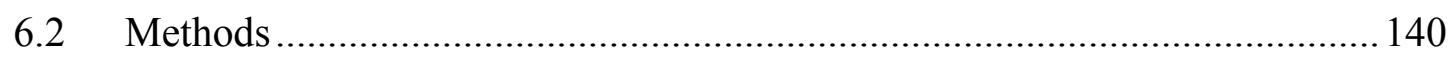

6.2.1 Proanthocyanidin staining in Swiss chard seeds ................................ 140

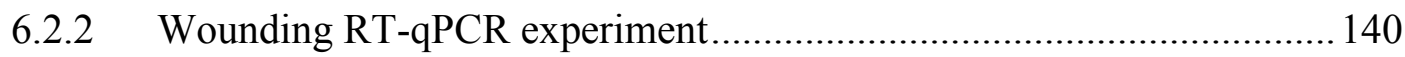

6.2.3 Methods for phylogenetic analyses................................................. 144

6.2.4 Transient gene expression analyses ................................................ 145

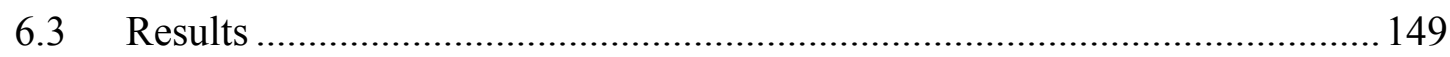

6.3.1 Swiss chard seeds contained proanthocyanidins................................. 149

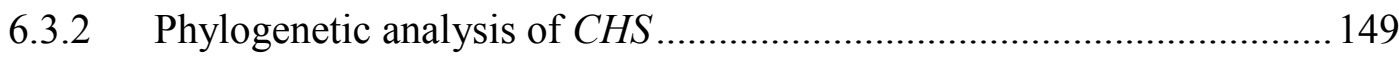

6.3.3 Flavonoid and betalain biosynthetic gene expression after wounding ... 149

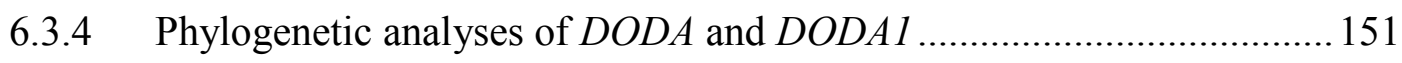

6.3.5 Transient betalain biosynthetic gene expression in Antirrhinum ............ 151

6.3.6 Transient betalain biosynthetic gene expression in Swiss chard ............ 153

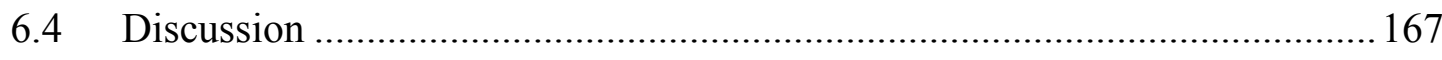

6.4.1 Regulation of betalain biosynthetic genes in response to wounding ...... 167

6.4.2 Comparison of DODA1 and DODA function..................................... 169

6.4.3 Biosynthetic control of betalains in yellow and white Swiss chard........ 170

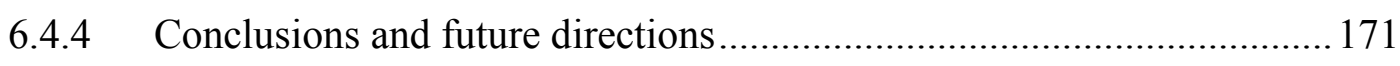

Chapter 7: General discussion ............................................................................173

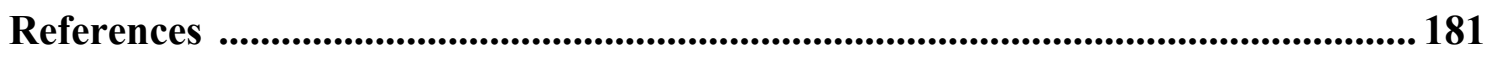

Appendices ....................................................................................................................... 199 


\section{List of figures}

Figure 1.1 Anthocyanin and betalain chemical structures

Figure 1.2 Comparison of betalain and anthocyanin pigmented flowers....................... 3

Figure 1.3 Observations of red pigments in fungal species ......................................

Figure 1.4 Evidence for possible light induction of betalain biosynthesis..................... 9

Figure 1.5 Diagram of putative transport routes for anthocyanin/proanthocyanidin vacuolar accumulation 12

Figure 1.6 Simplified flavonoid biosynthetic pathway .......................................... 16

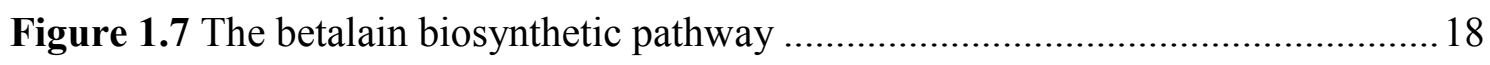

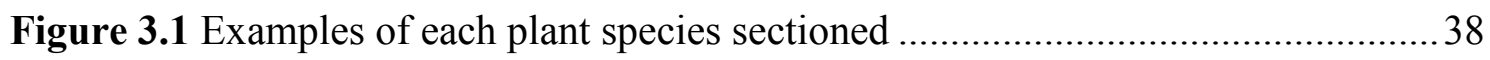

Figure 3.2 Betalain-containing lamina sections for each species............................. 44

Figure 3.3 Betalain-containing lamina sections for each species (continued) .............. 45

Figure 3.4 Betalain-containing petiole sections for each species................................46

Figure 3.5 Betalain-containing stem sections for each species .................................47

Figure 3.6 Betalain-containing stem sections for each species (continued) ..................48

Figure 3.7 Betalain-containing hypocotyl sections for each species ..........................49

Figure 3.8 Betalain-containing root sections for each species ..................................50

Figure 3.9 Observed pigmentation in specialised structures....................................51

Figure 3.10 Confocal analyses of the pigmented bodies in living stone lamina cross

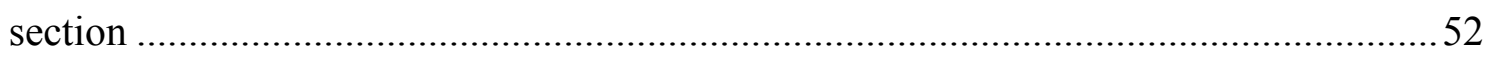

Figure 3.11 Differential localisation of betaxanthins and betacyanins across organ type and neighbouring cells

Figure 3.12 In situ absorptance measurements of individual cells in white, yellow, and purple Swiss chard

Figure 3.13 Representative chromatograms from HPLC analyses of white Swiss chard 
Figure 3.14 Representative chromatograms from HPLC analyses of yellow Swiss chard 56

Figure 3.15 Representative chromatograms from HPLC analyses of purple Swiss chard 57

Figure 3.16 Total betalain pigment concentration $\left(\mathrm{mg} \mathrm{g}^{-1} \mathrm{DW}\right)$ in five organs from purple and yellow Swiss chard 58

Figure 3.17 Proportion of betalamic acid in total HPLC peak area for five different organs of purple and yellow Swiss chard 59

Figure 3.18 Chromatograms from LC-MS analyses for whole plant extracts of purple, yellow, orange and red Swiss chard. 60

Figure 3.19 Betaxanthin compounds identified using LC-MS and HPLC analyses...... 62

Figure 3.20 Betacyanin compounds identified using LC-MS and HPLC analyses 63

Figure 3.21 Betacyanin compounds identified using LC-MS and HPLC analyses (continued) 64

Figure 4.1 Illustration of the four feeding methods trialled for L-DOPA and cyclo-DOPA to DOD-6 plants .83

Figure 4.2 Diagrams of Salk Institute PCR screening methods used for genotyping DOD-6 x $t$ t12 .84

Figure 4.3 Steps carried out in the genetic crossing of DOD-6 with the $t t$ mutants 85

Figure 4.4 Columbia and DOD-6 inflorescences fed with $10 \mathrm{mM}$ L-DOPA for DOD line selection 90

Figure 4.5 White light and blue light micrographs of filter paper feeding of $10 \mathrm{mM}$ L-DOPA to DOD-6 seedlings and mature plants 91

Figure 4.6 DOD-6 plants fed with L-DOPA + cyclo-DOPA using the filter paper method

Figure 4.7 Plants in hydroponic solution fed with additional water, $1 \mathrm{mM}$ cyclo-DOPA only, or $1 \mathrm{mM}$ L-DOPA $+1 \mathrm{mM}$ cyclo-DOPA 93

Figure 4.8 Filter tip feeding of $1 \mathrm{mM}$ L-DOPA to Columbia and DOD-6 seedlings ....94 Figure 4.9 Liquid culture feeding of DOD-6 seedlings with water, $1 \mathrm{mM}$ L-DOPA, and $1 \mathrm{mM}$ L-DOPA + cyclo-DOPA with $2 \mathrm{mM}$ ascorbic acid 95 
Figure 4.10 Visual selection of DOD-6 x PAP1-5 plants

Figure 4.11 Slow germination and growth of DOD-6 x PAP1-5 plants compared with DOD-6

Figure 4.12 Anthocyanin induction in DOD-6, tt19 and DOD-6 x tt19 seedlings germinated in water or $3 \%(\mathrm{w} / \mathrm{v})$ sucrose liquid culture .98

Figure 5.1 Illustration of betaxanthin transport hypotheses 105

Figure 5.2 Betaxanthin localisation in (A) Columbia and (B) DOD-6 plants following L-DOPA feeding 110

Figure 5.3 Anthocyanin localisation in DOD-6 x PAP1-5

Figure 5.4 Anthocyanin and betalain co-localisation in L-DOPA fed DOD-6 x PAP1-5

Figure 5.5 Co-localisation of anthocyanins and betalains in DOD-6 seedlings by anthocyanin induction and L-DOPA feeding.

Figure 5.6 Betaxanthin autofluorescence quenching in the presence of betacyanins and anthocyanins

Figure 5.7 Confocal analyses of betaxanthin accumulation in L-DOPA fed DOD-6 hypocotyl tissue.

Figure 5.8 Maximum projection of whole L-DOPA fed DOD-6 hypocotyl cells under confocal microscopy 116

Figure 5.9 Betaxanthin accumulation and autofluorescence in L-DOPA fed DOD-6 x tt12 inflorescences

Figure 5.10 Betaxanthin accumulation and autofluorescence in L-DOPA fed DOD-6 x tt19

Figure 5.11 Transverse sections of L-DOPA fed DOD-6 x $t t 12$ inflorescence stalks and lamina

Figure 5.12 Transverse sections of L-DOPA fed DOD- 6 x tt19 inflorescence stalks

Figure 5.13 Comparison of betaxanthin accumulation in L-DOPA fed DOD-6 with DOD-6 x $t$ t19 seedlings. 
Figure 5.14 Another example of betaxanthin cytoplasmic accumulation in L-DOPA fed DOD-6 x tt19 seedlings. 122

Figure 5.15 Betaxanthin accumulation in vanadate (VAN) treated DOD-6 seedlings under white and blue light. 123

Figure 5.16 Vanadate (VAN) affected betaxanthin accumulation in the vacuoles of L-DOPA fed DOD-6 seedlings 124

Figure 5.17 DOD-6 fed with L-DOPA and various transport inhibitors 125

Figure 5.18 DOD-6 x PAP1-5 seedlings treated with pyrimethamine (PYR) 126

Figure 5.19 Epidermal sections of DOD-6 x PAP1-5 treated with PYR and fed L-DOPA with PYR treatment. 127

Figure 5.20 Illustration of the results from the betalain transport experiments 130

Figure 6.1 Betalain biosynthetic pathway 138

Figure 6.2 Flowers of nivea Antirrhinum majus (chs mutant) 147

Figure 6.3 Swiss chard tissues used in transient gene expression assays 148

Figure 6.4 $0.3 \%(\mathrm{w} / \mathrm{v})$ DMACA-HCl staining (blue) indicating the presence of proanthocyanidins in wild type Columbia Arabidopsis thaliana and Swiss chard seeds

Figure 6.5 Phylogenetic analysis of $C H S$ with similar genes 155

Figure 6.6 Pigment accumulation patterns in wounded red- and white-petioled Swiss

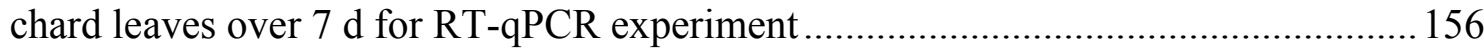

Figure 6.7 Flavonoid biosynthetic genes monitored in RT-qPCR ............................ 157

Figure 6.8 Betalain biosynthetic genes monitored in RT-qPCR .............................. 158

Figure 6.9 Relative fold change (RT-qPCR) of flavonoid biosynthetic genes from $0 \mathrm{~h}$ in wounded red- and white-petioled Swiss chard leaves 159

Figure 6.10 Normalised relative quantity (NRQ) of flavonoid biosynthetic genes after wounding treatment on red- and white-petioled Swiss chard leaves 160

Figure 6.11 Relative fold change (RT-qPCR) of betalain biosynthetic genes from $0 \mathrm{~h}$ in wounded red- and white-petioled Swiss chard leaves 161 
Figure 6.12 Normalised relative quantity (NRQ) of betalain biosynthetic genes after wounding treatment on red- and white-petioled Swiss chard leaves 162

Figure 6.13 Phylogenetic analysis (Maximum Likelihood) comparing beet DODA and $D O D A 1$ with $D O D$ genes from other betalain taxa 163

Figure 6.14 Transient betalain gene expression on Antirrhinum majus dorsal petals vacuum infiltrated with water and $10 \mathrm{mM}$ L-DOPA 164

Figure 6.15 Transient gene expression of CYP76AD1 + GFP and GFP only constructs on petioles of yellow Swiss chard. 165

Figure 6.16 Transient expression of betalain biosynthetic genes in white Swiss chard petioles 166

\section{List of tables}

Table 1.1 Localisation of anthocyanin pigments in different vegetative organs 7

Table 3.1 Location of betalains observed within tissues of plants sectioned. 43

Table 3.2 Betalain compounds identified using LC-MS in purple, yellow, orange and red Swiss chard plants 61

Table 3.3 Absolute quantity of compounds identified with HPLC from five organs of purple Swiss chard 65

Table 3.4 Absolute quantity of compounds identified with HPLC from five organs of yellow Swiss chard. .66

\section{Abbreviations}

$35 S$

3-MA

$A$

$\mathrm{ABC}$

Amp

ATP

$\mu \mathrm{AU}$
$35 S$ promoter from the cauliflower mosaic virus

3-methyladenine

absorbance

ATP-binding cassette

ampicillin

adenosine triphosphate

absorbance units used in HPLC and LC-MS 
AVI anthocyanic vacuolar inclusion

bc betacyanin

BDM 2, 3-butanedione monoxime, myosin inhibitor

BFA brefeldin A, golgi budding inhibitor

bHLH basic helix-loop-helix transcription factor

bp base pairs

bx betaxanthin

cDNA complementary DNA

CoA Coenzyme A

cyclo-DOPA cyclo-dihydroxyphenylalanine

DIC differential interference contrast, confocal image

DNA deoxyribonucleic acid

dNTP deoxyribonucleotide

DOPA dihydroxyphenylalanine

DTT dithiothreitol

DW dry weight

EDTA ethylenediaminetetraacetic acid

ER endoplasmic reticulum

EST expressed sequence tag

FW fresh weight

$\mathrm{F}_{\mathrm{x}} \quad$ filial generation, $\mathrm{x}$ denotes generation number

gDNA genomic DNA

GOI gene of interest

GSH glutathione

HM homozygous

HPLC high-performance liquid chromatography

$\mathrm{HZ}$ heterozygous

i.d. internal diameter

IPTG isopropyl- $\beta$-D-thiogalactopyranoside

JTT Jones-Taylor-Thornton, substitution model, phylogenetics

Kan kanamycin

$\mathrm{kb} \quad$ kilobases

LB Luria Bertani (agar or broth)

LC-MS liquid chromatography-mass spectrometry 


\begin{tabular}{|c|c|}
\hline $\mathrm{LT}$ & Ligandin Transport model \\
\hline$\lambda_{\max }$ & maximum absorbance \\
\hline Milli-Q & water which was purified using Milli-Q Ultrapure system \\
\hline ML & maximum likelihood, heuristic method, phylogenetics \\
\hline MOPS & 3-(N-morpholino) propanesulfonic acid \\
\hline MS & Murashige and Skoog, tissue culture media \\
\hline$n$ & sample size \\
\hline NNI & nearest neighbour interchange, model parameter, phylogenetics \\
\hline NRQ & normalised relative quantity \\
\hline$O C S$ & terminator from the Agrobacterium tumefaciens octopine synthase gene \\
\hline PA & proanthocyanindin \\
\hline PCR & polymerase chain reaction \\
\hline PVC & pre-vacuolar compartment \\
\hline PYR & pyrimethamine, MATE inhibitor \\
\hline R2R3MYB & MYB containing only the second and third repeats \\
\hline rpm & revolutions per minute \\
\hline RT-qPCR & real-time quantitative PCR \\
\hline S.E. & standard error \\
\hline sp./spp. & species \\
\hline Spec & spectinomysin \\
\hline $\mathrm{TE}$ & tris-EDTA buffer \\
\hline $\mathrm{T}_{\mathrm{m}}$ & melting temperature \\
\hline TGN & trans-Golgi network \\
\hline$t t$ & transparent testa mutation which affects Arabidopsis thaliana seed coat \\
\hline $\mathrm{T}_{\mathrm{x}}$ & filial generation from a transgenic line, $\mathrm{x}$ denotes generation number \\
\hline UV & ultra violet \\
\hline $\mathrm{v} / \mathrm{v}$ & volume/volume \\
\hline VAN & vanadate, ATPase inhibitor \\
\hline VT & Vesicular Transport model \\
\hline WD40 & WD-repeat protein \\
\hline $\mathrm{w} / \mathrm{v}$ & weight/volume \\
\hline WT & wild type \\
\hline X-gal & 5-bromo-4-chloro-3-indolyl galactopyranoside \\
\hline
\end{tabular}




\section{Abbreviations of proteins}

ABC ATP-binding cassette

$\mathrm{ABCC} 1 \quad$ Vitis vinifera $\mathrm{ABC}$ transporter

ACT actin

AM1 Vitis vinifera MATE transporter

AM3 Vitis vinifera MATE transporter

AN9 Petunia GST

ANR anthocyanidin reductase

ANS anthocyanidin synthase

AUS aureusidin synthase

BBS bibenzyl synthase

BGT betanin glucosyltransferase

BZ2 Zea mays GST

C4'GT chalcone 4'-O-glucosyltransferase

cDOPA GT cyclo-DOPA glucosyltransferase

CHI chalcone isomerase

CHS chalcone synthase

CYP76AD1 betalain cytochrome P450

DFR dihydroflavonol 4-reductase

DOD DOPA extradiol dioxygenase

EF2 elongation factor 2

F3GT UDP-glucose:flavonoid 3-O-glucosyltransferase

$\mathrm{F} 3 \mathrm{H} \quad$ flavanone-3-hydroxylase

FFT Flower Flavonoid Transporter

FLS flavonol synthase

FNS flavone synthase

GFP green fluorescence protein originally from Aequorea Victoria

GST glutathione $S$-transferease

GST3 Cyclamen GST

GS-X glutathione-activated membrane transport protein

GT glycosyltransferase

LAR leucoanthocyanidin reductase

MATE multidrug and toxic extrusion transporter 
MRP multidrug resistance-associated protein

MRP1 Arabidopsis thaliana multidrug resistance-associated protein

MRP3 Zea mays multidrug resistance-associated protein

MYB class of transcription factor

PAP1 Production of Anthocyanin Pigment 1

PPO polyphenoloxidase

StCS1 stilbenecarboxylate synthase 1

STS stilbene synthase

TT12 Arabidopsis thaliana Transparent Testa 12, MATE transporter

TT19 Arabidopsis thaliana Transparent Testa 19, GST

TYR tyronsine hydroxylating enzyme 


\section{Chapter 1: General introduction}

\subsection{Thesis background}

Plants typically have three main groups of pigments: chlorophylls, carotenoids and anthocyanins. While chlorophylls and carotenoids are universal, one group of plants do not produce anthocyanins and instead produce betalains. Betalain pigments are commonly referred to as the anthocyanin replacement. Widespread adoption of this replacement hypothesis may be the reason why research into betalain biosynthesis has been somewhat overlooked, compared to anthocyanin biosynthesis, which is one of the most well studied biosynthetic pathways in plants. Structural details of betalain compounds were obscure until the middle of last century (Steglich \& Strack, 1990). Elucidation of these structures led betalain compounds to be called 'nitrogenous anthocyanins', which incorrectly implies structural similarities between anthocyanins (Figure 1.1A) and betalains (Figure 1.1B and 1.1C) (Musso, 1979). Soon after, Mabry and Dreiding (1967) coined the term 'betalain' to associate these pigments with their derivative, betalamic acid (Figure 1.1D), originally identified from red beet (Beta vulgaris). During this project, several experiments were designed to test the betalain replacement hypothesis. It was found that, although betalains and anthocyanins have similar tissue localisation, and that betalains can be transported by anthocyanin transporters, key regulatory control may differ.<smiles></smiles>

Figure 1.1 Anthocyanin and betalain chemical structures. (A) Anthocyanin; (B) Betacyanin; (C) Betaxanthin; (D) Betalamic acid. R denotes a glycosyl unit. 


\subsection{Plant pigments: betalains and anthocyanins}

Anthocyanins and betalains give plants a range of vibrant red and purple colours. These pigment types can appear visually similar (Figure 1.2); they are localised to similar organ types (Lee \& Collins, 2001), are both stored in the vacuole, and both have high antioxidant and radical scavenging activities (Cai et al., 2003; Gandía-Herrero et al., 2010; Strack et al., 2003). Despite all these similarities, anthocyanins and betalains are mutually exclusive, meaning no plant has been found with both pigment types (Stafford, 1994). Other than their roles in colouring flowers and fruits, to attract pollinators and seed dispersers, these pigments are also commonly found in vegetative organs. In vegetative tissues, however, these pigments have no unified functional role. A number of physiological roles have been hypothesised for these pigments. The most supported physiological roles are: protecting sensitive photosynthetic tissue through a light screening role, and preventing free-radical damage induced by abiotic stressors through their antioxidant activity (see reviews by Hatier and Gould (2009) and Stafford (1994)).

Betalain are nitrogen-containing pigments with two classes, betacyanins (violet, $\lambda_{\max }=$ $536 \mathrm{~nm}$ ) and betaxanthins (bright yellow, $\lambda_{\max }=470 \mathrm{~nm}$ ). These plant pigments are found within the Order Caryophyllales, except in the Caryophyllaceae and the Molluginaceae families that produce anthocyanins instead (Strack et al., 2003). There are only around 55 known betalain compounds, compared with over 550 known anthocyanin molecules (Stintzing \& Carle, 2007).

There has been interest in the usage of betalain pigments in foodstuffs (Stintzing \& Carle, 2007). Their high antioxidant capacity has been suggested to increase the human health benefits of food (Kapadia et al., 1996; Tesoriere et al., 2004). Apart from the health benefits of betalains, these pigments are used as a natural food dye. Betalains are water soluble and more stable than anthocyanins over a broad pH range (Azeredo, 2009; Stintzing \& Carle, 2004; Strack et al., 2003), and their tinctorial (dyeing) strength is up to $3 \mathrm{x}$ higher than anthocyanins (Stintzing $\&$ Carle, 2007). Other research areas are their biosynthesis and the reason why plants produce betalains instead of anthocyanins (Brockington et al., 2011). These aspects are discussed in later sections of this chapter. 

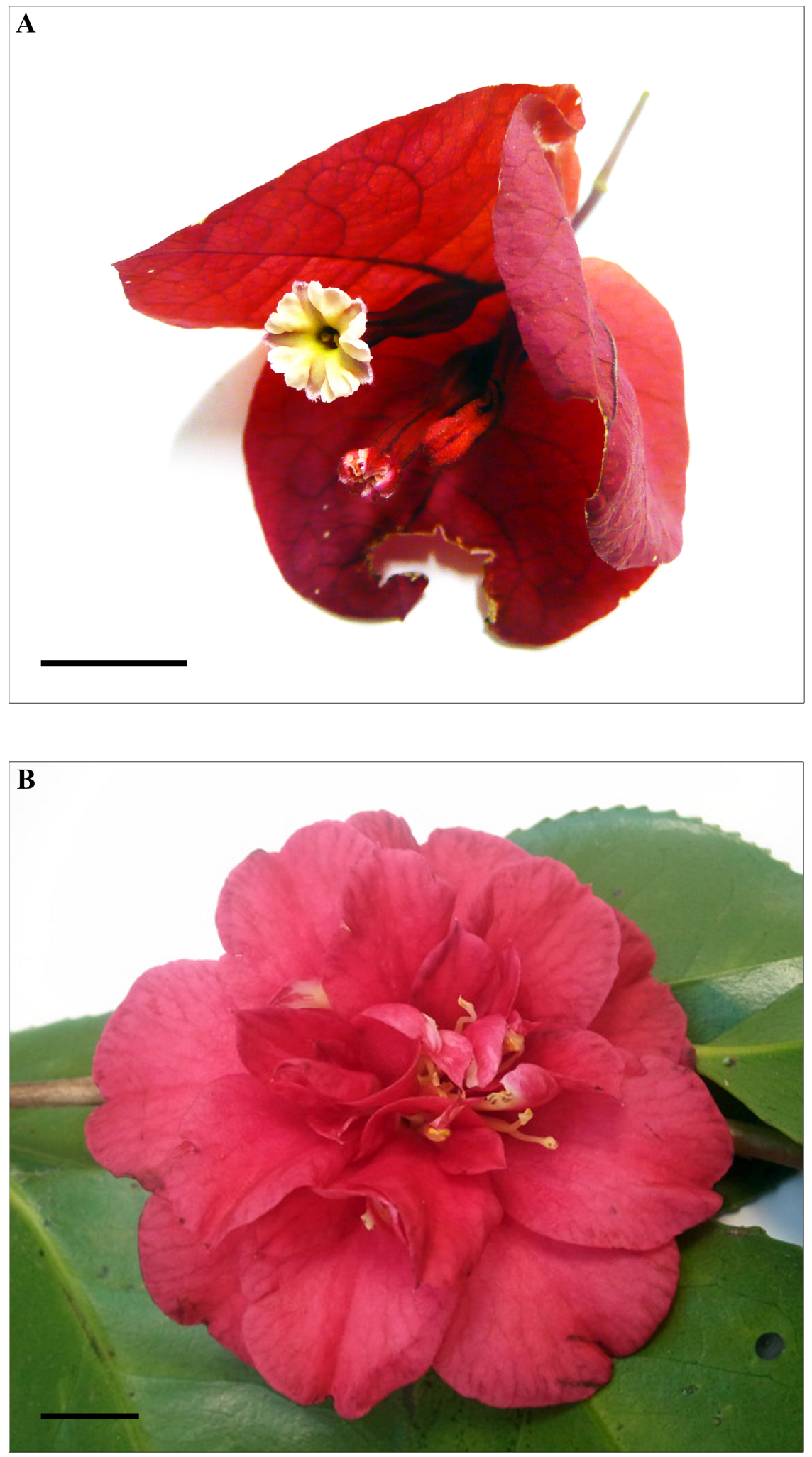

Figure 1.2 Comparison similarly coloured betalain and anthocyanin pigmented flowers. (A) Betalain pigmented Bougainvillea spectabilis, and (B) anthocyanin pigmented Camellia sp. Scale bars $=10 \mathrm{~mm}$. 


\subsection{Evolution and mutual exclusivity}

The evolutionary reason behind the mutual exclusivity of anthocyanin and betalain pigments in plants is a mystery (Strack et al., 2003). Betalains have evolved at least twice, once within the fungal lineage Basidiomycetes (Figure 1.3), and once within the flowering plant Order Caryophyllales. The presence of betalain pigments in fungi is likely from convergent evolution, given that a key enzyme in betalain biosynthesis, DOPA extradiol dioxygenase (DOD), in the fungal species Amanita muscaria is structurally different to the plant DOD (Christinet et al., 2004).

Within the Caryophyllales, several hypotheses were suggested to explain the phenomenon of anthocyanin and betalain mutual exclusivity, as discussed in Brockington et al. (2011). Ehrendorfer (1976) proposed that mutual exclusivity resulted from betalains evolving as an ecological adaptation for a changing environment, to arid and semi-arid conditions. Later, the colonisation of less marginal habitats then led to the reversal of this trait in the two anthocyanin-producing Caryophyllales families, Caryophyllaceae and Molluginaceae. An alternative hypothesis by Clement and Mabry (1996) suggests that the ancestor of the Caryophyllales contained both pigments, and subsequently there was the loss of one or the other pigment type. The hypothesis for co-pigmentation, however, does not have any supporting evidence. No taxa have been found with both pigment types, although, it is possible that this exists in unexplored taxa or extinct. No mechanisms were proposed by Clement and Mabry (1996) to account for the loss of either pigment, although recently, it has been suggested that one could be selectively lost due to lack of evolutionary pressure to retain redundant functions (Grotewold, 2006). Certainly, the evolutionary history of pigmentation in the Caryophyllales is complex. In the review by Brockington et al. (2011), the origin of betalains in the Caryophyllales was discussed. By reviewing recent molecular phylogenetic analyses and using character mapping of the Caryophyllids, two different evolutionary histories for the origin of betalains were proposed. The first proposal is that betalains originated twice within the Caryophyllales (with secondary betalain evolution in Amaranthaceae), and with two reversals to the anthocyanic state. The second proposal is that betalains originated once in the Caryophyllales, with three reversals to the anthocyanic condition. Both of these evolutionary histories support the 

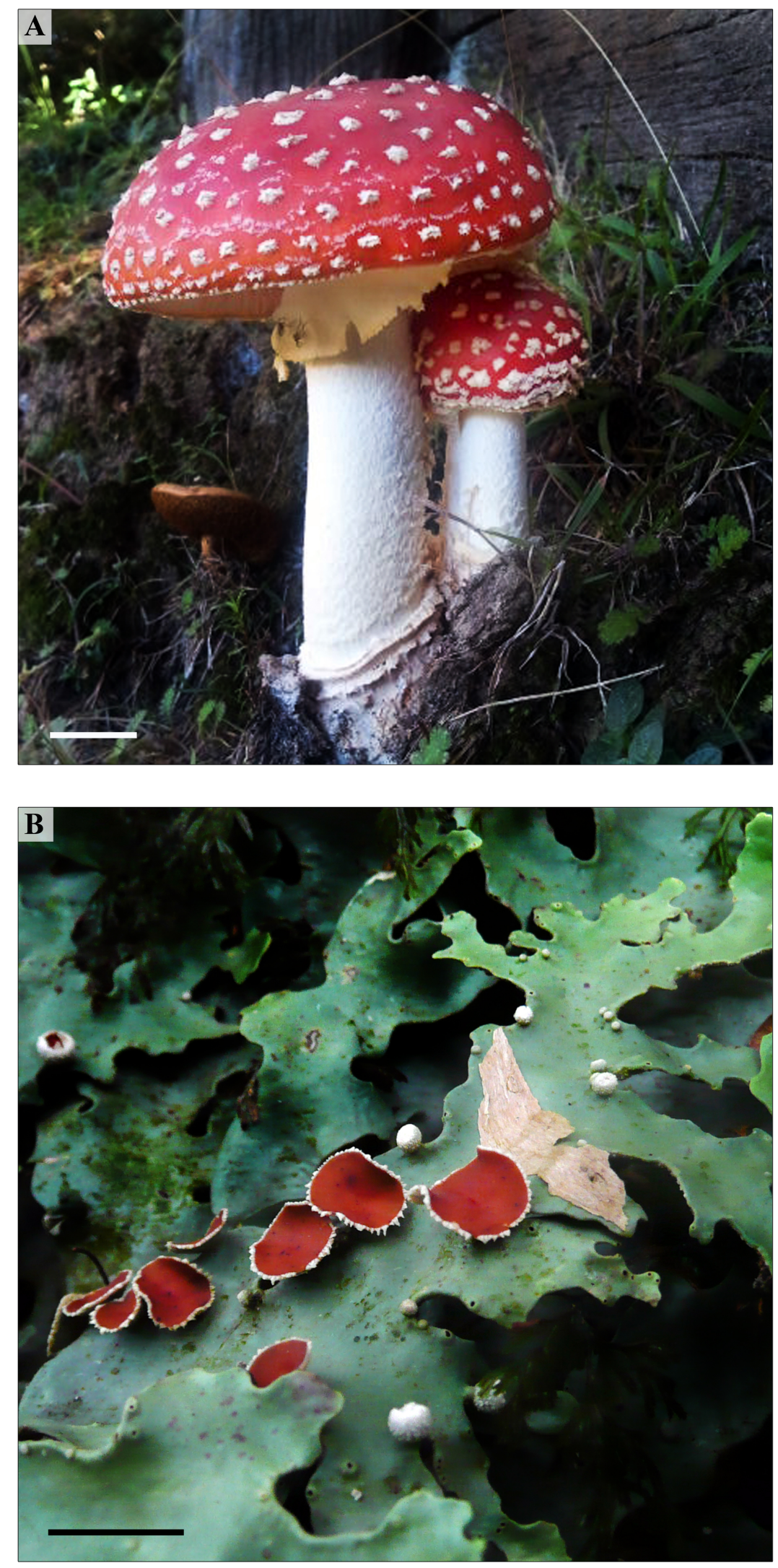

Figure 1.3 Observations of red pigments in fungal species. Betalain pigments present in (A) the caps of Amanita muscaria, and potentially in (B) the fruiting bodies of a fungal symbiont in an unidentified lichen species. Scale bars $=10 \mathrm{~mm}$. 
hypothesis that betalains are the anthocyanin replacement. However, further details on possible mechanisms for this evolution are needed.

\subsection{Localisation}

The external appearance of anthocyanin and betalain pigments in plants is one of the main reasons why betalains are said to replace anthocyanins. Both pigments are found in flowers, fruits and multiple vegetative organs. Given the external similarities between anthocyanins and betalains, as well as the prevalence of anthocyanin-producing species, the internal distribution and localisation of betalains has been largely overlooked. Numerous studies have analysed the localisation of anthocyanins in different vegetative organs (Table 1.1). Within these organs anthocyanin pigments have been found in epidermal, ground parenchyma and vascular tissues. For betalains, only a few studies have looked into where these pigments are localised in vegetative tissues (Lee \& Collins, 2001; Mosco, 2012; Petrus-Vancea et al., 2010). In these studies, betalain localisation in the leaves and petioles are similar to that found for anthocyanins, which supports the replacement hypothesis. However, betalain localisation studies are not comprehensive enough to directly compare with what is known about anthocyanin localisation. Analyses of more betalain-producing species from different families, and the sectioning of other organs such as hypocotyls, stems and roots in these plants are required for better comparisons.

In anthocyanin-producing plants, red pigmentation patterns in vegetative tissues are similar in distantly related species (Gould \& Quinn, 1999; Lee \& Collins, 2001). Phylogenetic analyses on pigment localisation in these species, determined that the patterns observed were only weakly explained by phylogenetic relatedness (Lee \& Collins, 2001). This suggests that similarities in appearance between anthocyanin- and betalain-producing plants may be due to similar biological functions. Biosynthesis of both pigment types seems to be controlled by developmental timing and both are induced by external factors such as abiotic and biotic stress, as discussed below. Therefore, the spectral similarities between pigments could be due to similar physiological roles in plants. 
Table 1.1 Localisation of anthocyanin pigments in different vegetative organs.

\begin{tabular}{|c|c|c|}
\hline Organ & Tissue type & Source \\
\hline Lamina & BS, EB, ED, HY, MP, MS, PH & $\begin{array}{l}\text { Blank (1947); Gould and Quinn } \\
\text { (1999); Kytridis and Manetas } \\
\text { (2006); Lee and Collins (2001); Li } \\
\text { et al. (2008); Neill and Gould } \\
\text { (2000); Nozzolillo and McNeill } \\
\text { (1985) }\end{array}$ \\
\hline Petiole & $\mathrm{CO}, \mathrm{EP}, \mathrm{GP}, \mathrm{PH}$ & $\begin{array}{l}\text { Li et al. (2008); Nozzolillo and } \\
\text { McNeill (1985) }\end{array}$ \\
\hline Stem & CX, EP, PH, PI & $\begin{array}{l}\text { Blank (1947); Gould et al. (2010); } \\
\text { Li et al. (2008); Nozzolillo (1973); } \\
\text { Nozzolillo and McNeill (1985) }\end{array}$ \\
\hline Hypocotyl & CX, EP & $\begin{array}{c}\text { Blank (1947); Nozzolillo (1973); } \\
\text { Nozzolillo and McNeill (1985); } \\
\text { Thakur and Nozzolillo (1978); } \\
\text { Troyer (1964) }\end{array}$ \\
\hline Root & $\mathrm{CX}, \mathrm{EP}, \mathrm{PH}, \mathrm{RC}$ & $\begin{array}{c}\text { Blank (1947); Solangaarachchi and } \\
\text { Gould (2001); Thakur and } \\
\text { Nozzolillo (1978) }\end{array}$ \\
\hline
\end{tabular}

Key: BS bundle sheath, CO collenchyma, CX cortex, EB abaxial epidermis, ED adaxial epidermis, EP epidermis, GP Ground parenchyma, HY hypodermis, MP palisade mesophyll, MS spongy mesophyll, PH phloem, PI pith, RC root cap. 


\subsection{Betalain and anthocyanin production is induced by similar factors}

Anthocyanin and betalain pigmentation is induced by a variety of factors, many of which are shared by both pigments types. These include developmental as well as a range of abiotic and biotic signals. Anthocyanins and betalains often appear transiently in growing, young tissues (Lee \& Collins, 2001). Developmental timing of their synthesis may relate to the production of certain hormones during growth and development. Several different hormones induce anthocyanin and betalain biosynthesis. These include ethylene (Cao et al., 2012; El-Kereamy et al., 2003), abscisic acid (Jeong et al., 2004) and methyl jasmonate (Bhuiyan \& Adachi, 2003; Cao et al., 2012; Suresh et al., 2004). Girod and Zrÿd (1991) also found that in Beta vulgaris cell cultures, the ratio of auxin $(2,4-\mathrm{D})$ to cytokinin (6-BAP) was important for regulating the biosynthesis of betacyanins.

As both anthocyanins and betalains are coloured pigments, their interaction with light has received the most attention. Light plays a key part in the regulation of the production of anthocyanins (Stafford, 1994; Steyn et al., 2002). Anthocyanin pigments not only often require light for synthesis, but their biosynthesis is also enhanced by light (Albert et al., 2009; Solangaarachchi \& Gould, 2001). Genes in the anthocyanin biosynthetic pathway may be switched on by ultra-violet (UV) light, blue light (Ahmad et al., 1995; Wang et al., 2012), or far-red light (Shin et al., 2013) photoreceptors. These photoreceptors in turn induce the expression of transcription factor genes such as R2R3MYBs that regulate anthocyanin biosynthesis. In contrast to anthocyanins, light is not essential for betalain biosynthesis, although high light exposure can enhance the quantity of pigment produced (de Nicola et al., 1975; Piattelli et al., 1969; Vogt et al., 1999). The role of light exposure in the betalain pathway is not well known. However, it may relate to similar photoreceptor mechanisms (betalain light induction Figure 1.4; Kochhar et al. (1981)). Like anthocyanins, betalain biosynthesis is particularly enhanced by UV or blue light (Kishima et al., 1995; Kochhar et al., 1981; Zrÿd \& Christinet, 2004). Although, in Suaeda salsa betacyanin accumulation was greater in plants that were placed in the dark, which degraded when the plants were exposed to blue light (Chang-Quan \& Tao, 2006). 

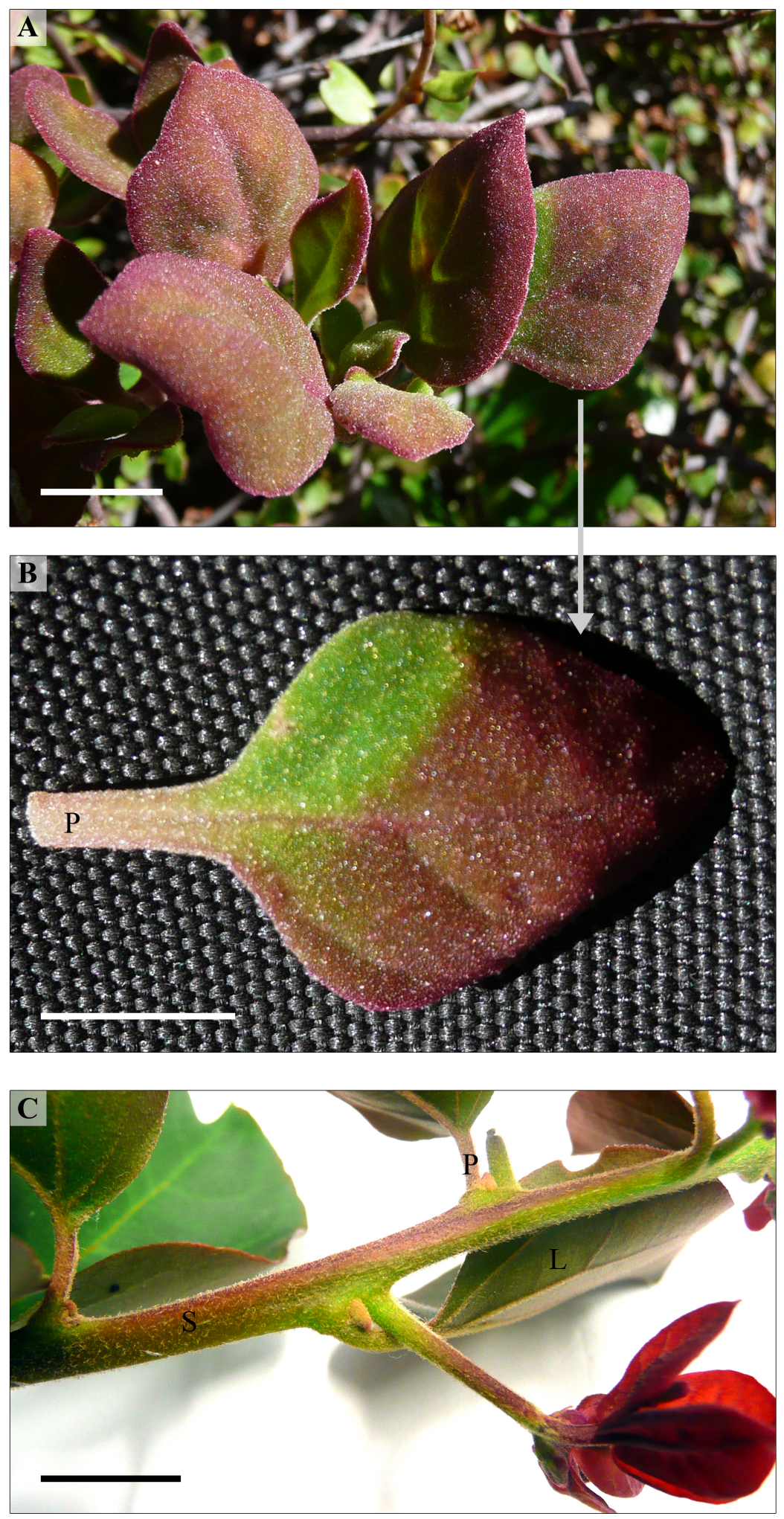

Figure 1.4 Evidence for possible light induction of betalain biosynthesis in (A and $\mathbf{B})$ Tetragonia tetragoniodes (New Zealand spinach) and (C) Bougainvillea spectabilis. The leaf (B) removed from plant (A) was red, except for the area that was shaded by another leaf. The upper halves of bougainvillea stems and petioles exposed to high light developed red pigmentation, whereas, the bottom halves were green. Key: L leaf; P petiole; S stem. Scale bars $=10 \mathrm{~mm}$. 
Other known factors that induce pigment production include: physical wounding (Gould et al., 2002; Reyes \& Cisneros-Zevallos, 2003; Sepulveda-Jimenez et al., 2005; Sepúlveda-Jiménez et al., 2004), as well as exposure to low temperatures (Christie et al., 1994; Hayakawa \& Agarie, 2010; Powell \& Weedin, 2004; Stiles et al., 2007) and salinity (Chalker-Scott, 2002; Stintzing \& Carle, 2004; Wang et al., 2008).

Exposure to high light and the other factors above can cause physiological stress within plants. Red pigmentation in vegetative tissues is hypothesised to reduce physiological stress through two main functional roles (Chalker-Scott, 1999; Hatier \& Gould, 2009): (1) via a light screening role in tissues exposed to long periods of photoinhibitory light, and (2) through the sequestration of free radicals produced in response to stress.

Both betacyanins and anthocyanins absorb in the green spectrum of visible light (Gandía-Herrero et al., 2010; Steyn et al., 2002). It is hypothesised that the presence of these red pigments in epidermal tissues screens out harmful levels of incident light, thus reducing photoinhibitory damage. Numerous studies have demonstrated enhanced photosynthetic ability in anthocyanic versus acyanic plants (Gould et al., 2010; Hughes et al., 2005; Manetas, 2006; Merzlyak \& Chivkunova, 2000; Page \& Towers, 2002), and in betalain-producing plants (Nakashima et al., 2011).

The antioxidant capacity of both anthocyanins and betalains has been well-documented (Steyn et al., 2002; Stintzing \& Carle, 2004; Wang et al., 1997). These pigments may function by reducing the damage of free radicals, produced by stresses such as physical wounding. Free radicals can damage cellular membranes and proteins, including the photosystem complexes in chloroplasts (Foyer et al., 1994; Solovchenko \& Merzlyak, 2008). Gould et al. (2002) demonstrated that, after wounding, anthocyanin-enriched regions of Pseudowintera colorata leaves had enhanced rates of $\mathrm{H}_{2} \mathrm{O}_{2}$ scavenging, compared to green regions. Studies comparing in vivo antioxidant activity of red versus green leaves in betalain-producing plants, suggest that betalains may reduce oxidative stress in a similar way (Chen et al., 2013; Hayakawa \& Agarie, 2010).

Given that similar factors induce anthocyanin and betalain pigment accumulation, it would be interesting to investigate whether anthocyanins and betalains have similar mechanisms that regulate their biosynthesis. 


\subsection{Transport and storage}

Betalain and anthocyanin pigments are both stored in the vacuole. The vacuole can be the largest organelle in plant cells, occupying up to $90 \%$ of the cell volume (Kenyon $\&$ Black, 1986; Marty, 1999; Matile, 1978; Moriyasu \& Hillmer, 2000). The main functions of the vacuole are osmotic regulation of cell volume, compartmentation of enzymes, and storage of ions and metabolites, including secondary metabolites. These functions are mediated via the tonoplast by directly controlling the transport of compounds in and out of the vacuole, or indirectly as a barrier of the vacuolar contents from the cytoplasm (Matile, 1978). Flavonoids, including anthocyanins, are synthesised in a complex of metabolic enzymes on the cytoplasmic side of endoplasmic reticulum (ER) (Winkel-Shirley, 1999). Recent research suggests that betalain biosynthesis occurs in a similar location (Gandía-Herrero \& García-Carmona, 2012). After biosynthesis, however, the mechanisms for anthocyanin transport to the vacuole are not fully understood (Tanaka et al., 2008), and mechanisms for betalain transport are completely unknown (Grotewold, 2006).

Flavonoid derivatives are transported from the ER to different locations. Anthocyanins, proanthocyanidins (PAs), flavonol and flavone glycosides and most other flavonoids accumulate in vacuolar compartments, however, some flavonoid compounds are either secreted from the cell or accumulate in cell wall (Grotewold, 2004). It is thought that anthocyanins and PAs are directly transported from the ER to vacuole, as they are not processed through the trans-Golgi network (Poustka et al., 2007). Transport mechanisms involved in flavonoid vacuolar transport and sequestration have been identified in a number of plant species, although there is no unified transport hypothesis. In the review by Grotewold and Davies (2008), these mechanisms were divided into two models for flavonoid trafficking: the Vesicular Transporter (VT) and the Ligandin Transporter (LT) models. Each of these models, as described by Grotewold and Davies (2008) and the possible transport mechanisms involved are discussed below.

The VT model describes mass-transport of anthocyanin and PA compounds by vesicles (Figure 1.5A). Vesicles are thought to sequester flavonoid compounds from the ER or the cytoplasm and directly transport them to the vacuole. Circular bodies containing anthocyanins were observed in the cytoplasm of Eustoma grandiflorum Grise. 


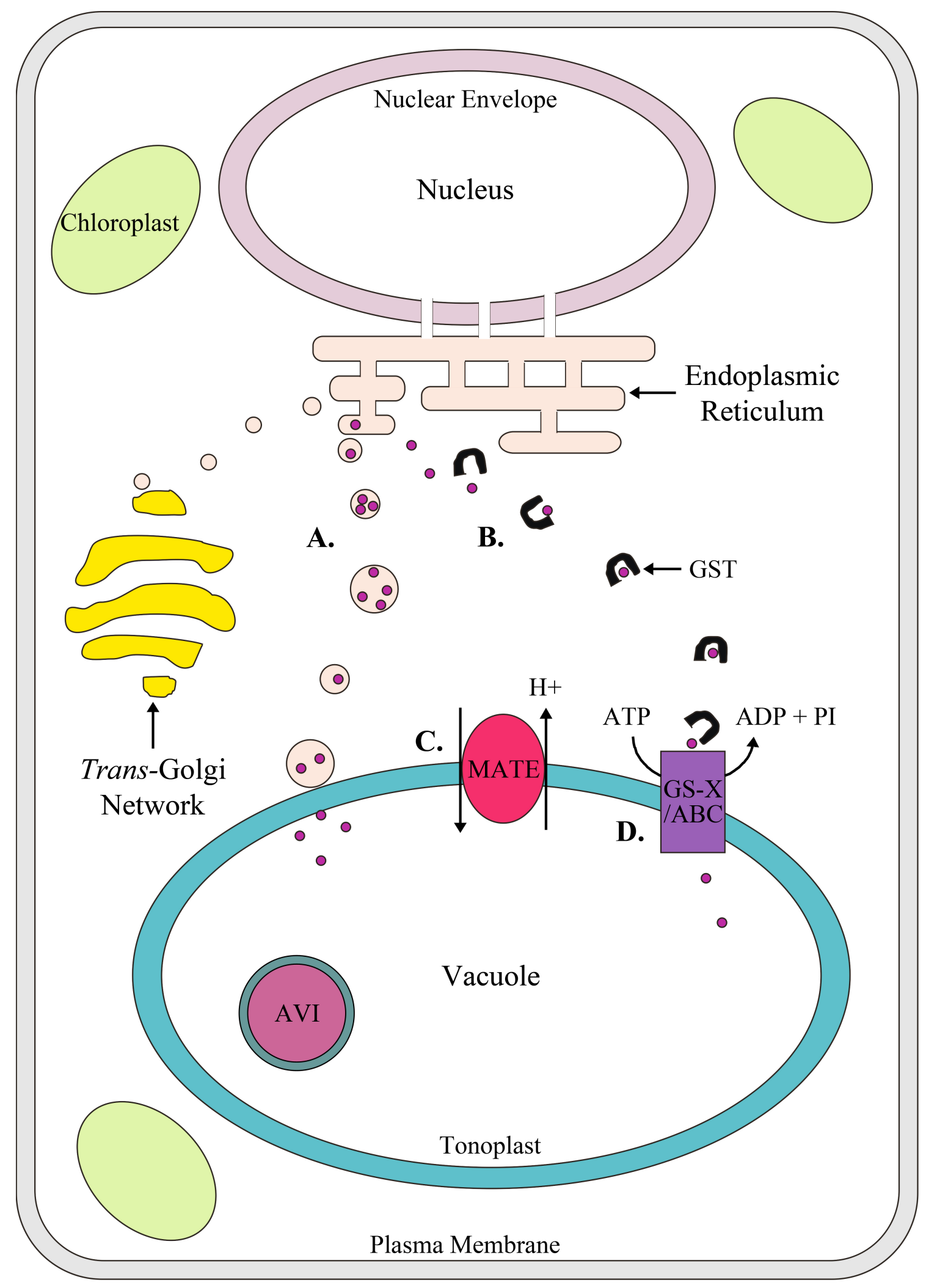

Figure 1.5 Diagram of putative cellular transport routes for anthocyanin/proanthocyanidin vacuolar accumulation. (A) Direct transport via vesicles; (B) Glutathione $S$-transferase (GST) carrier protein binding to anthocyanin/proanthocyanidin molecules; (C) Multidrug and toxic extrusion (MATE) transporter on tonoplast or vesicles; (D) Glutathione/ATP-binding cassette (GS-X/ABC) pump on tonoplast. AVI anthocyanic vacuolar inclusion. 
(lisianthus; Zhang et al. (2006)), Arabidopsis thaliana (Poustka et al., 2007), Zea mays L. (maize; Grotewold et al. (1998)), Brassica oleracea L. cv. Niggerhead (cabbage; Small and Pecket (1982)) and Vitis vinifera L. (grape; Gomez et al. (2011)). In the tapetum cells of developing pollen of several Brassica species, flavonoids were deposited in tapetosomes from ER-derived vesicles (Hsieh \& Huang, 2005, 2007). This suggests that VT of other flavonoids, such as anthocyanins, may also have ER-derived vesicles.

The LT model describes the transport of flavonoid compounds via binding to glutathione $S$-transferase (GST) transporters (Figure 1.5B). GSTs are a large family of transporters, and usually function by attaching a glutathione (GSH) molecule to the substrate, which is then recognised by a glutathione transport (GS-X) pump (Marrs, 1996; Theodoulou, 2000). The involvement of GST-like proteins in flavonoid transport have been demonstrated in maize (BZ2; Marrs et al. (1995)), Petunia spp. (petunia; AN9; Alfenito et al. (1998)), Arabidopsis (TT19; Kitamura et al. (2004)), grape (GST4; Gomez et al. (2011)) and Cyclamen spp. (cyclamen; GST3; Kitamura et al. (2012)). Disruption of GST-like proteins in these plants resulted in accumulation of anthocyanins in the cytoplasm and in vesicle-like structures (Gomez et al., 2011; Marrs et al., 1995; Sun et al., 2012), in addition to a reduction of PA accumulation in Arabidopsis seed vacuoles (Kitamura et al., 2004). Precise involvement of GSTs in anthocyanin transport is unclear because anthocyanin-GSH conjugates have not been detected (Marrs et al., 1995). Rather than attaching a GSH molecule, GSTs are suggested to be carrier proteins that bind directly to anthocyanin or PA molecules, and deliver them to a dedicated membrane transporter (Mueller et al., 2000).

The mechanisms of the VT and LT models appear independent. Although, as Grotewold and Davies (2008) pointed out, these models may be unified through their cross-membrane transport. Both models require that compounds cross at least one membrane: the vesicle membrane in the VT model, or the tonoplast in the LT model. Two classes of trans-membrane transport proteins have been implicated in flavonoid vacuolar transport: the multidrug and toxic extrusion (MATE) transporter (Figure 1.5C), and the ATP-binding cassette (ABC) transporter (Figure 1.5D).

MATE transporters are part of a large family that function with an $\mathrm{H}^{+}$-gradient-dependent mechanism via an $\mathrm{H}^{+}$antiport (Yazaki, 2005). Involvement of 
MATE transporters in vacuolar accumulation of PAs has been demonstrated in Arabidopsis (TT12; Debeaujon et al. (2001)) and grape (AM1 and AM3; Gomez et al. (2009)). Localisation of this transporter was detected in the vesicle (Gomez et al., 2011) and tonoplast membrane fractions (Debeaujon et al., 2001). Glycosylated epicatechins are preferentially transported (Zhao \& Dixon, 2010), but it has been demonstrated that MATE transporters may also contribute a small amount to anthocyanin transport (Debeaujon et al., 2001).

ABCs are a class of tonoplast pumps which use the energy of ATP to drive transport across the membrane (Theodoulou, 2000; Yazaki, 2005). ABC transporters, or multidrug resistance-associated proteins (MRPs, which are a specific class of ABCs), were identified to be involved in the transport of anthocyanins in Arabidopsis (MRP1; Lu et al. (1997)), maize (MRP3; Goodman et al. (2004)) and grape (ABCC1; Francisco et al. (2013)). In maize and grape, anthocyanin vacuolar accumulation was dramatically reduced through blocking the function of these transporters. In addition, the grape ABC transporter $\mathrm{ABCC} 1$ was dependent on the presence of GSH compounds, without the formation of an anthocyanin-GSH conjugate. This indicates that, at least in grape, the $\mathrm{ABC}$ transporter may be an ATP-activated GS-X pump. ABC transporters may also be involved in the VT model. Arabidopsis treated with a general ATPase inhibitor vanadate (Van) increased the number of anthocyanic vacuolar inclusions (AVIs) that formed (Poustka et al., 2007). Enhanced AVI formation may have occurred due to incomplete fusion of anthocyanin-containing vesicles with the vacuole.

As the species analysed to date, such as maize, Arabidopsis and grape, appear to involve both the VT and LT mechanisms in flavonoid transport, it is possible that these models are one in the same (as discussed in Grotewold and Davies (2008)). The role of ligandin transporters may be through aiding the transport of compounds across vesicle membranes and/or stabilising these compounds during transport in vesicles. This would explain the close association between GSTs and membranous structures observed in grape subcellular localisation assays (Gomez et al., 2011). The involvement of different membrane transport proteins is yet to be defined, but these may participate in the transport of different compound types, such as aglycones versus glycosylated anthocyanins, or anthocyanins versus PAs. Although different species indicated differential localisation of cross-membrane transporters, accurate subcellular 
localisation of transporters to particular membranes is difficult. Detection of these transporters on the tonoplast does not exclude occurrence elsewhere in the cell, such as on the transport vesicles or on the ER.

\subsection{Biosynthetic pathways}

The biosynthetic pathways of anthocyanin and betalain pigments are different. They have different starting amino acid precursors and do not share any biosynthetic enzymes.

\subsubsection{Flavonoid biosynthesis}

Flavonoids are produced in the phenylpropanoid pathway (Grotewold, 2006). The core flavonoid pathway is shown in Figure 1.6. Details of the key biosynthetic steps are taken from several reviews (Davies, 2009; Tanaka et al., 2008; Xie \& Dixon, 2005). The pathway begins with a condensation reaction of three molecules of malonyl-CoA (derived from the acetate pathway) and one molecule of coumaroyl-CoA (derived from L-phenylalanine) by chalcone synthase (CHS) [1], to produce a light yellow chalcone. A side branch from the chalcones, to produce bright yellow aurones, involves aureusidin synthase (AUS) [2] and chalcone 4'-O-glucosyltransferase (C4'GT). Alternatively, chalcone can be converted into the colourless flavanone naringenin by isomerisation, which is enhanced by chalcone isomerase (CHI) [3]. This compound can be used by flavone synthase (FNS) [4] to produce flavones, or be hydroxylated at the $\mathrm{C}_{3}$ position by flavanone-3-hydroxylase (F3H) [5] to give unpigmented dihydroflavonols.

After the production of dihydroflavonols, the pathway branches into the production of flavonols, anthocyanins and PAs. Flavonols are produced by the action of flavonol synthase (FLS) [6]. The anthocyanin/proanthocyanidin branch continues by the reduction of dihydroflavonol by dihydroflavonol 4-reductase (DFR) [7], to yield a colourless leucoanthocyanidin. Anthocyanidin synthase (ANS) [8] then oxidises the $\mathrm{C}_{3}$ position to produce coloured anthocyanidins. Further modifications of the anthocyanidins can proceed via glycosylation, which is most commonly carried out by UDP-glucose:flavonoid 3-O-glucosyltransferase (F3GT) [9]. The production of PAs involves leucoanthocyanidin reductase (LAR) [10] which produces catechins; and anthocyanindin reductase (ANR) [11] for the creation of epicatechins. PAs can also polymersise to form condensed tannins. 


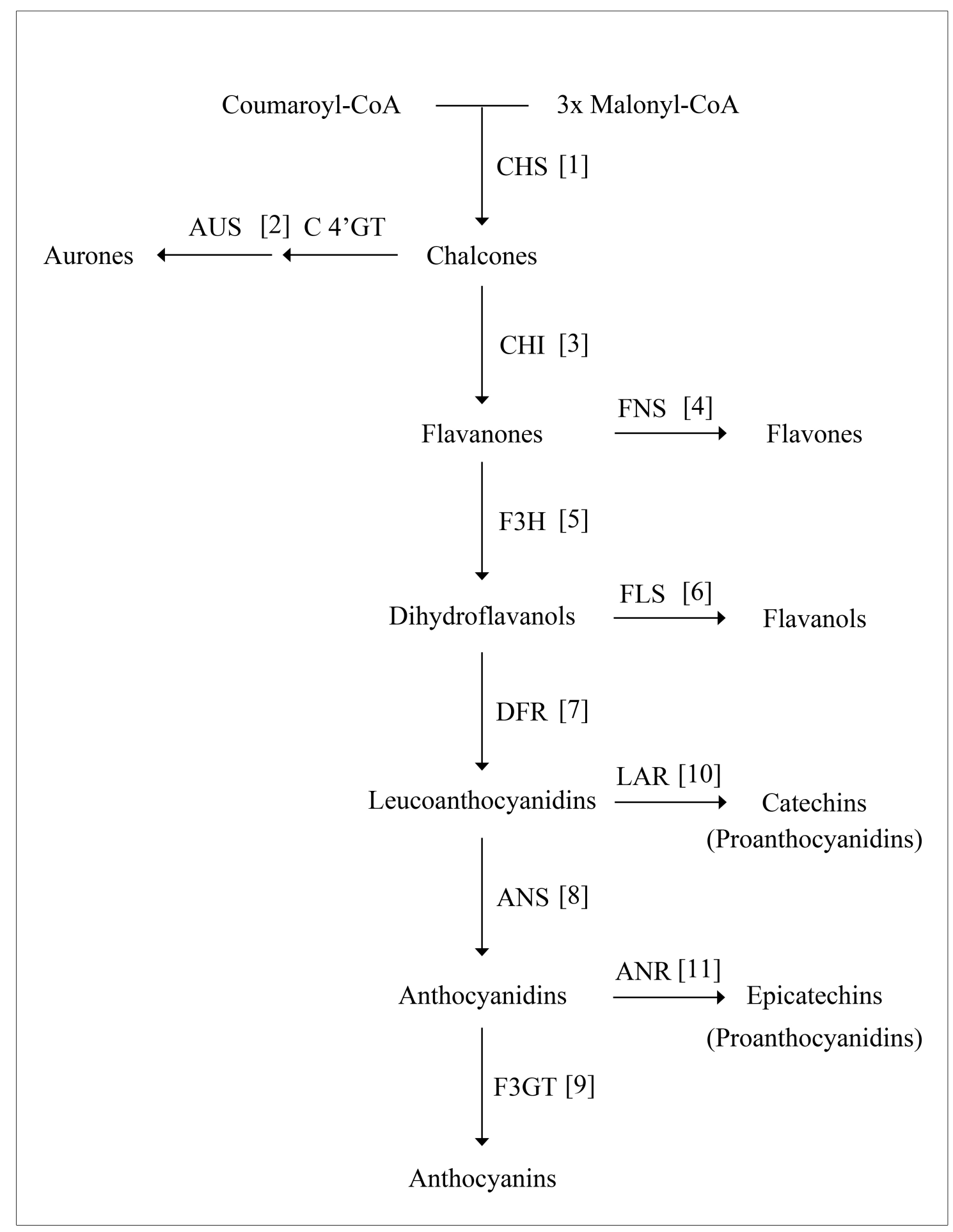

Figure 1.6 Simplified flavonoid biosynthetic pathway. Numbers refer to enzyme reactions described in the text. Enzyme abbreviations: ANR anthocyanidin reductase, ANS anthocyanidin synthase, AUS aureusidin synthase, C4'GT chalcone 4'-O-glucosyltransferase, CHI chalcone isomerase, CHS chalcone synthase, DFR dihydroflavonol 4-reductase, F3GT UDP-glucose:flavonoid 3-O-glucosyltransferase, F3H flavanone-3-hydroxylase, FLS flavonol synthase, FNS flavone synthase, LAR leucoanthocyanidin reductase. 
Many flavonoid compounds are found in betalain-accumulating plants, but not the anthocyanins (Grotewold, 2006). Given that the flavonoid biosynthetic genes would, therefore, need to be functional within these plants, it was assumed that the lack of anthocyanins is due to non-functional genes at the end of the anthocyanin pathway. However, many of these plants produce PAs in their seeds, which would require DFR to be functional and, depending on the type of PA, also ANS. This is what Shimada et al. $(2004,2005)$ found in spinach and common purselane. Furthermore, the promoter regions of $D F R$ and $A N S$ were unable to bind common anthocyanin transcription factors (Shimada et al., 2007). It was, therefore, suggested, that these genes might have tissue-specific expression, to enable the production of PAs in seeds, but inhibit the production of anthocyanins in vegetative and floral tissues.

\subsubsection{Betalain biosynthesis}

Much of what is known about betalain biosynthesis was discovered in the last two decades. Details of betalain biosynthesis were taken from several studies and reviews (Gandía-Herrero \& García-Carmona, 2013; Grotewold, 2006; Hatlestad et al., 2012; Stafford, 1994; Steiner et al., 1999). Betacyanin and betaxanthin compounds share the same structural backbone of betalamic acid, and differ in the molecule that is attached to it by conjugation (Gandía-Herrero et al., 2010). Betalains have a simple biosynthetic pathway, especially when compared with anthocyanins. The current betalain pathway model is illustrated in Figure 1.7.

Betalain biosynthesis begins with the amino acid tyrosine. Betacyanins require two tyrosine molecules and betaxanthins require one. Tyrosine is hydroxylated by an enzyme (TYR) [12] to form DOPA. There is some debate around what enzyme is actually involved in this reaction (as reviewed in Gandía-Herrero and García-Carmona (2013)). In the fungal species, Amanita muscaria, this step is catalysed by a tyrosinase (Mueller et al., 1996). In plants, betacyanin accumulation was correlated with increases in transcript and activity of a tyrosinase (or polyphenoloxidase) in Phytolacca americana (Joy et al., 1995), Suaeda salsa (Wang et al., 2007), Portulaca grandiflora and Beta vulgaris (Steiner et al., 1999). Despite the correlation between tyrosinase activity and betalain accumulation, no one has cloned a betalain-related tyrosinase gene, or convincingly demonstrated that one is involved in plant betalain synthesis. More recently, Hatlestad et al. (2012) postulated that a cytochrome P450 may carry out this 


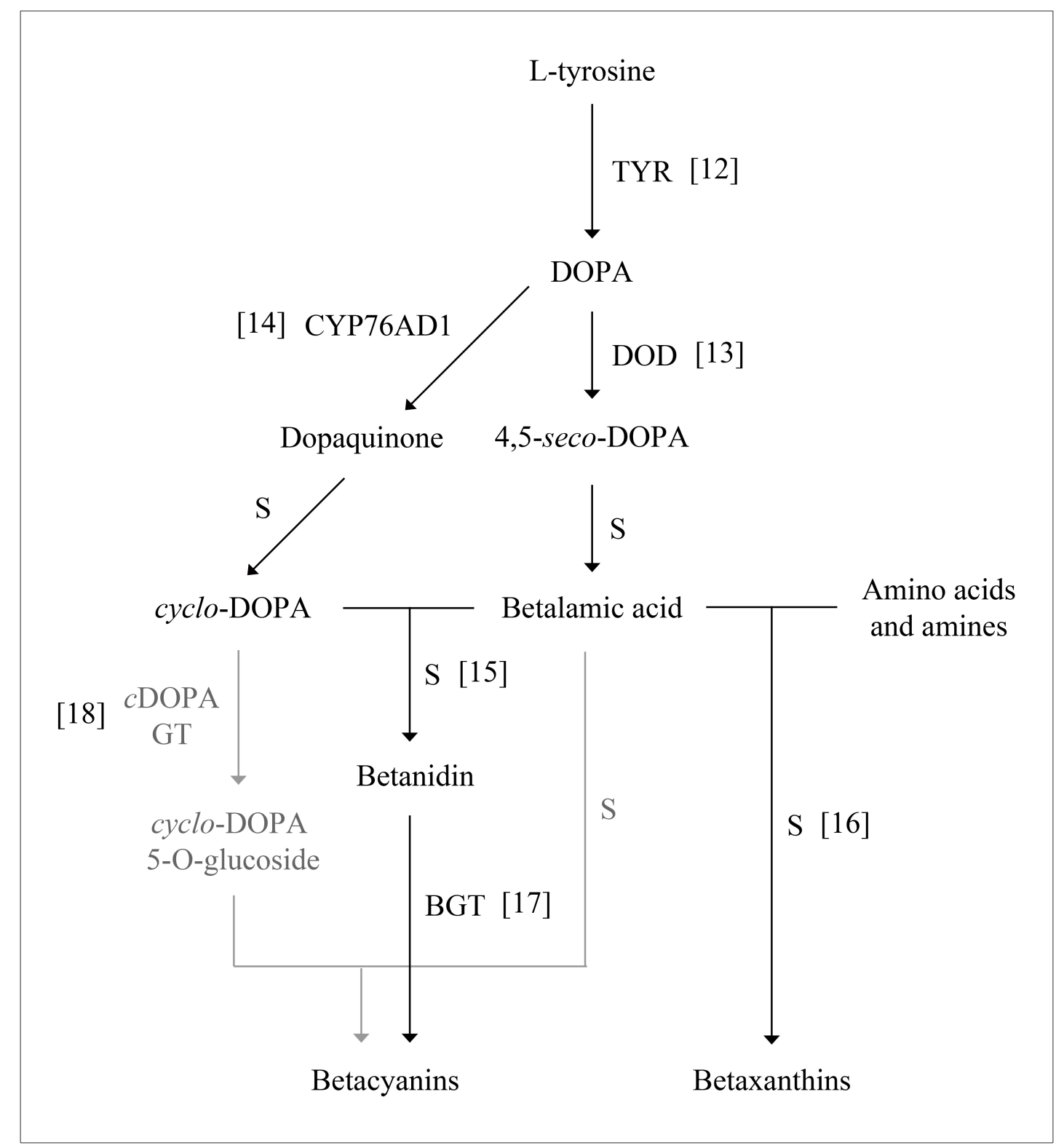

Figure 1.7 The betalain biosynthetic pathway. Numbers refer to enzyme reactions described in the text. Enzyme abbreviations: BGT betanidin glucosyltransferase, cDOPA GT cyclo-DOPA glucosyltransferase, CYP cytochrome P450, DOD 4,5-DOPA extradiol dioxygenase, TYR tyrosine hydroxylating enzyme. $\mathrm{S}$ is a spontaneous reaction. Grey pathway illustrates alternative glucosylation route. 
hydroxylation step in Beta vulgaris, in addition to catalysing the conversion of DOPA to cyclo-DOPA (as discussed below). In the production of melanin in animals, a single tyrosinase enzyme performs both a tyrosine hydroxylation and a DOPA oxidation reaction (Winder \& Harris, 1991). However, as discussed in Hatlestad et al. (2012), a number of betaxanthin only mutants exist in several different betalain-producing species. The independent production of betaxanthins from betacyanins suggests that there may be two different enzymes controlling these reactions. Therefore, a question mark remains over this biosynthetic step in the pathway.

Following the synthesis of DOPA, betalamic acid is formed from DOPA by a 4,5-DOPA extradiol dioxygenase (DOD) [13], which catalyses the oxidation of DOPA to form the intermediate 4,5-seco-DOPA. This compound then experiences spontaneous cyclisation to betalamic acid. DOD homologues have been found in plants whether or not they produce betalains (Christinet et al., 2004), such as in Arabidopsis (Genbank accession NM_117597; Mayer et al. (1999)), which naturally produces anthocyanins. $D O D$ genes in betalain-synthesising species may have evolved from these DOD homologues (Christinet et al., 2004).

Another key molecule in betalain biosynthesis, as it is needed for betacyanin synthesis, is cyclo-DOPA. It is synthesised from betalamic acid by a cytochrome P450 (CYP76AD1) [14]. CYP76AD1 synthesises dopaquinone from betalamic acid which undergoes spontaneous cyclisation to form cyclo-DOPA. Conjugation reactions between betalamic acid with cyclo-DOPA [15], or other amino acids/amines [16] are thought to occur spontaneously rather than through an enzyme-catalysed reaction (Grotewold, 2006; Schliemann et al., 1999; Strack et al., 2003). The final betacyanin molecule is usually glycosylated and may also be acylated (Azeredo, 2009; Tanaka et al., 2008). These structures are glycosylated on one or two free hydroxyl groups of cyclo-DOPA, at the C-5 or C-6 position (Steglich \& Strack, 1990). Glycosylation of betacyanin can occur on the betanidin molecule with betanidin glucosyltransferase (BGT) [17] (Heuer \& Strack, 1992; Heuer et al., 1996) or on the cyclo-DOPA molecule with cyclo-DOPA glucosyltransferase ( $c$ DOPA GT) before conjugation with betalamic acid [18] (Sasaki et al., 2005a; Sasaki et al., 2004; Sasaki et al., 2005b; Sepulveda-Jimenez et al., 2005). 


\subsection{Biosynthetic regulation}

Regulation of flavonoid pigment production is primarily through the transcriptional regulation of biosynthetic genes (Davies et al., 2012). A number of models for flavonoid biosynthetic regulation have been established in plants such as maize, Arabidopsis, petunia and Antirrhinum majus (Koes et al., 2005; Martin et al., 1991; Winkel-Shirley, 2001). In these model plants, conserved regulatory elements with sequence-specific DNA binding have been identified (Hichri et al., 2011). Anthocyanin biosynthesis is directly regulated by a complex of R2R3MYB and basic helix-loop-helix (bHLH) transcriptional factors, a WD40-type protein and their interaction (Tanaka et al., 2008). As discussed in Davies et al. (2012), in some species anthocyanin biosynthetic genes are also actively repressed by R2R3MYBs. These repressors target motifs in the C-terminal domains of genes and inhibit their transcription (Aharoni et al., 2001; Jin et al., 2000; Tamagnone et al., 1998).

Involvement of these regulatory elements in response to light, fruit ripening or in flower colour development are particularly well characterised (see reviews by Davies et al. (2012), Davies and Schwinn (2003), Hichri et al. (2011), Petroni and Tonelli (2011)). The biosynthetic regulation after other induction mechanisms, such as wounding, are less well characterised, but some components have been identified. Wounding induces up-regulation of flavonoid genes CHS (Djordjevic et al., 1997; Richard et al., 2000), F3H (Singh et al., 2008), and DFR (Peters \& Constabel, 2002). A number of MYB transcription factors may specifically regulate these genes in response to wounding (Cheong et al., 2002; Mellway et al., 2009).

Little is known about the biosynthetic regulation of betalains. Gandía-Herrero and García-Carmona (2013) suggested that betalain pigment production may be controlled by the up-regulation of biosynthetic genes. With only three main enzyme-controlled steps in the betalain pathway, the possible points for biosynthetic regulation are few. Correlations have been made between increased tyrosinase transcripts and the accumulation of betalains, in developing fruits of Phytolacca americana (Joy et al., 1995) and during flower development in Portulaca grandiflora (Kishima et al., 1991). Similarly, the transcript expression levels for DOD and CYP76AD1 directly corresponded to the total amount of betaxanthin and betacyanin compounds, respectively, in different Beta vulgaris lines (Hatlestad et al., 2012). Post-transcriptional 
regulation of the betalain biosynthetic enzyme tyrosinase was also suggested by GandíaHerrero and García-Carmona (2013). The beet tyrosinase may be regulated by tyrosinase activating agents and proteases that transform a latent enzyme into an active one (Gandía-Herrero et al., 2005c, 2005d). Methylation of betalain biosynthetic genes has may also play a key role in the repression of genes encoding enzymes in betacyanin synthesis (Zrÿd \& Christinet, 2004).

Genetic control of colour has been narrowed down to several loci in Portulaca grandiflora and Beta vulgaris (Zrÿd \& Christinet, 2004). Most of these loci are now determined to be biosynthetic genes in the betalain pathway, but other uncharacterised loci may also be involved in regulation of these genes. Alternatively, given that anthocyanins and betalains are induced by many of the same factors, their biosynthetic pathways may use the same regulatory mechanisms. MYBs often regulate more than one pathway (Hichri et al., 2011), therefore, it is possible that transcription factors similar to those in the flavonoid pathway may be involved in the regulation of betalain biosynthesis.

\subsection{Pigmentation mutants in pigment biosynthesis research}

Many discoveries of anthocyanin biosynthesis were made through the use of mutants, which have reduced or completely blocked anthocyanin biosynthesis. Anthocyanin mutants have been characterised for a number of species including A. majus, petunia, maize and Arabidopsis (Mol et al., 1998). Many of these mutations affect flavonoid enzymes, but some affect regulatory loci. The main benefit for using mutants in the determination of gene function is that they result in visible phenotypes. Arabidopsis transparent testa $(t t)$ mutants are good examples of this (Pourcel et al., 2012). The phenotype produced by a $t t$ mutation is a pale seed coat. Through the use of $t t$ mutants, a large number of genes involved in PA biosynthesis have been identified.

There are not as many known betalain pigment mutants to work with. Two examples of characterised betalain pigment mutations are: the $D O D$ mutant in white-petaled Portulaca grandiflora (Christinet et al., 2004) and the CYP76AD1 mutant in yellow beet (Beta vulgaris subsp. vulgaris cv. 'Golden Globe') (Hatlestad et al., 2012). As a large amount of betalain biosynthetic research has been carried out on beet to date, the beet subspecies Swiss chard (Beta vulgaris subsp. cicla cv. 'Bright Lights') was chosen 
as a model plant for analysing biosynthetic regulation in this study. This species came in a wide variety of colours, which aided the comparison of betacyanin and betaxanthin biosynthesis.

\subsection{Thesis objectives}

The primary objective for this research was to investigate betalain localisation and biosynthesis to compare with the knowledge for anthocyanins. Betalains are called the anthocyanin replacement based upon their mutual exclusivity, similarities in appearance and from sharing similar factors which induce pigment production. Based upon this replacement hypothesis, tissue localisation and control of betalain biosynthesis should be similar to that of anthocyanins. The following hypotheses were tested in this thesis:

1. Betalains and anthocyanins pigment the same tissue and cell types.

2. Betalains and anthocyanins share transport mechanisms from site of synthesis to storage in the vacuole.

3. Biosynthesis of betalains and flavonoids uses the same regulatory mechanisms.

Experiments were designed to test these hypotheses, which form the basis of this thesis. These experimental objectives were as follows:

- Determine the localisation of betalain pigment and compare with what is known about anthocyanin tissue localisation.

- Develop Arabidopsis as an artificial betalain plant model for testing betalain transport and storage questions.

- Test the limits of anthocyanin and betalain co-localisation in vivo.

- Analyse betalain transport in the artificial betalain plant model using known flavonoid transport mutants and chemicals which inhibit anthocyanin transport.

- Investigate betalain and flavonoid biosynthetic regulation in Swiss chard following wounding induction.

- Test betalain biosynthetic gene function in Swiss chard using transient gene expression assays. 


\section{Chapter 2: General methods}

\subsection{Chemicals}

Chemicals and reagents were sourced from Merck Millipore, Sigma-Aldrich or Roche, unless otherwise specified.

\subsection{Media, buffers and solutions}

All culture media, buffers and solutions were prepared using Milli-Q water. Where appropriate, these were autoclaved at $120{ }^{\circ} \mathrm{C}$ and $100 \mathrm{kPa}$ for $20 \mathrm{~min}$ before use. For solutions that were not autoclaved, previously autoclaved Milli-Q water was used. All recipes for media, buffers and solutions are listed in Appendix 1.

\subsection{Plant growth conditions and treatments}

\subsubsection{Growth conditions}

Plants purchased at a mature age were maintained in the greenhouse until required. Plants grown from seed were sown onto moist soil and germinated in high humidity using a misting system on a heating bed in the greenhouse. Seedlings were subsequently grown in the greenhouse or within a growth chamber which was maintained at $22{ }^{\circ} \mathrm{C}$ with a $16 \mathrm{~h}$ photoperiod and $65 \%$ humidity.

\subsubsection{Vegetative tissue and seed surface sterilisation}

Vegetative tissues were surface sterilised before biolistic-mediated transformation experiments. Tissue was soaked in a $10 \%(\mathrm{v} / \mathrm{v})$ bleach $(5 \%(\mathrm{w} / \mathrm{v})$ sodium hypochlorite) solution containing a drop of Tween 20 for $10 \mathrm{~min}$. Tissue was then rinsed three times with water before being dried. Seeds were surface sterilised similar to vegetative tissues, but were initially rinsed with $70 \%(\mathrm{v} / \mathrm{v}) \mathrm{EtOH}$ before the bleach treatment. The bleach solution used for seeds was $50 \%(\mathrm{v} / \mathrm{v})$ bleach (5\% (w/v) sodium hypochlorite) with a drop of Tween 20. After $6 \mathrm{~min}$ in the bleach solution, seeds were rinsed with water three times before they were resuspended in water and placed in the dark at $4{ }^{\circ} \mathrm{C}$. 


\subsection{General microscopy details}

\subsubsection{Dissecting microscopy}

Whole plant tissues were photographed using a Leica M205 FA stereomicroscope with DC500 camera and fluorescence attachments. For autofluorescence microscopy, a narrow blue fluorescence filter cube was used. This filter had a peak of $470 \mathrm{~nm}$ with a $40 \mathrm{~nm}$ range $(450-490 \mathrm{~nm})$ and a $515 \mathrm{~nm}$ LP barrier filter.

\subsubsection{Compound microscopy}

Plant tissues were hand sectioned and mounted in water on glass slides. Sections were photographed using an Olympus Provis AX70 compound microscope with an Olympus DP70 camera and U-MWB fluorescence attachments. This microscope was equipped with a blue fluorescence filter cube with the same specifications as Section 2.4.1.

\subsubsection{Confocal microscopy}

Confocal microscopy was carried out at the Massey Microscopy \& Imaging Centre in Palmerston North using a Leica SP5 DM6000B confocal microscope. The confocal microscope was equipped with an argon laser, which emitted light at $488 \mathrm{~nm}$. The measured emission spectra were $498-560 \mathrm{~nm}$ for betaxanthins and $625-732 \mathrm{~nm}$ for chloroplasts.

\subsection{Primer design}

Primers were designed using DNASTAR Lasergene ${ }^{\circledR}$, Genomic Suite (ION Torrent), Geneious Pro 5.5.6 (BioMatters Ltd.), QuantPrime (Arvidsson et al., 2008), and PrimerQuest ${ }^{\circledR}$ (Integrated DNA Technologies). All primers were synthesised as a commercial service by Sigma-Aldrich. A list of primers used is included in Appendix 2.

\subsection{Nucleic acid extraction and manipulation}

\subsubsection{Genomic DNA extraction}

Genomic DNA (gDNA) extractions were carried out on fresh and frozen leaf tissue approximately the size of a $1.5 \mathrm{~mL}$ microcentrifuge lid. Tissue was ground to powder while frozen using blue plastic grinding pestles and liquid nitrogen. To each sample, $400 \mu \mathrm{L}$ of gDNA extraction buffer was added (Appendix 1.5.1) and vortexed for $5 \mathrm{~s}$. 
Samples sat at room temperature for at least $15 \mathrm{~min}$ and were spun at $14000 \mathrm{rpm}$ in a bench top microcentrifuge for $1 \mathrm{~min}$ before $300 \mu \mathrm{L}$ of supernatant was transferred to a new tube. An equal volume of buffered phenol/chloroform/IAA (Sigma-Aldrich) was added to each extract and vortexed until mixed. Samples were spun at $14000 \mathrm{rpm}$ for $3 \mathrm{~min}$ and the aqueous layer was transferred to a new tube. To each sample, $300 \mu \mathrm{L}$ of $100 \%$ isopropanol was added and mixed by gentle inversion. Extracts sat at room temperature for $5 \mathrm{~min}$ before being centrifuged at full speed for $5 \mathrm{~min}$. Supernatant was discarded and the pellet was washed with $70 \%(\mathrm{v} / \mathrm{v})$ EtOH. Tubes were inverted on tissues to dry. Genomic DNA was resuspended in $50 \mu \mathrm{L}$ TE buffer (Appendix 1.5.2) then stored at either $4{ }^{\circ} \mathrm{C}$ or $-20^{\circ} \mathrm{C}$.

\subsubsection{RNA extraction and complementary DNA (cDNA) synthesis}

Fresh or frozen tissue was ground in liquid nitrogen to powder and weighed out while frozen to a maximum of $100 \mathrm{mg}$ for the RNA extraction. RNA extractions used the RNeasy ${ }^{\circledR}$ Mini Plant kit (Qiagen) following the manufacturer's protocol. RNA was eluted from the column using $31 \mu \mathrm{L}$ of RNase free water. Following DNase I treatment, using the Deoxyribonuclease I kit from Invitrogen, $2 \mu \mathrm{g}$ of RNA from each sample was used in cDNA synthesis. Creation of cDNA used the Transcriptor First Strand cDNA Synthesis kit (Roche) and followed the manufacturer's protocol. Half of the RNA from each sample was used for cDNA synthesis, the other half as a Reverse Transcriptase (RT) control, which excluded the Transcriptor enzyme as a control for gDNA contamination. The cDNA samples and RT controls were diluted twenty fold unless otherwise specified, aliquoted into smaller volumes, and stored at either $-20{ }^{\circ} \mathrm{C}$ or $-80{ }^{\circ} \mathrm{C}$.

\subsubsection{Assessment of DNA and RNA quality and quantity}

\subsubsection{Gel electrophoresis}

Concentration and quality of nucleic acids was visualised using gel electrophoresis. $10 \mathrm{x}$ loading dye (Appendix 1.4.3) was added to polymerase chain reaction (PCR) products, plasmid restriction digests and gDNA at a $1 \mathrm{x}$ concentration before being loaded onto a 1 - $2 \%(\mathrm{w} / \mathrm{v})$ TBE agarose gel (Appendix 1.4.1). DNA gels were run in 1x TBE buffer (Appendix 1.4.1) at $120 \mathrm{~V}$ for $40 \mathrm{~min}$. Estimates of DNA size were made by comparing the DNA samples against known DNA size standards (1 kb Plus ladder, Invitrogen). To 
analyse RNA quality, $10 \times$ RNA loading dye (Appendix 1.4.4) was added to RNA samples at a $1 \times$ concentration before being loaded onto a MOPs gel with formaldehyde (Appendix 1.4.2). RNA gels were run in a $1 \mathrm{x}$ MOPs buffer (Appendix 1.4.2) at $80 \mathrm{~V}$ for $1 \mathrm{~h}$. All gel-separated nucleic acids were visualised by ethidium bromide fluorescence using UV-illumination on a Gel Doc ${ }^{\mathrm{TM}} \mathrm{XR}+$ System (BioRad Laboratories Inc.).

\subsubsection{Spectrophotometric evaluation of nucleic acids}

The purity and quantity of DNA and RNA in samples was measured using a Nanodrop Spectrophotometer (ND-1000, Thermo Scientific). $1 \mu \mathrm{L}$ of samples were pipetted onto the spectrophotometer and measured at wavelengths $230 \mathrm{~nm}, 260 \mathrm{~nm}$ and $280 \mathrm{~nm}$. The Nanodrop software used measurements at these wavelengths to calculate purity and concentration of nucleic acids.

\subsubsection{PCR conditions}

PCR was routinely used for a number of applications including genotyping, cloning, screening bacterial colonies and gene isolation. PCR was performed using DNA polymerases, Taq polymerase (Roche), KAPA HiFi DNA Polymerase (Kapa Biosystems) and KAPA 2G DNA polymerase (Kapa Biosystems). PCR cycling parameters and reaction volumes were modified depending on primer pair, product size and target sequence. Primer pairs are listed in Appendix 2. A typical PCR reaction and cycling conditions are outlined below.

$12 \mu \mathrm{L}$ Sterile $\mathrm{H}_{2} \mathrm{O}$

$0.5 \mu \mathrm{L} \quad 10 \mathrm{mM}$ dNTP mix

$2 \mu \mathrm{L} \quad 10 \mu \mathrm{M}$ forward strand primer

$2 \mu \mathrm{L} \quad 10 \mu \mathrm{M}$ reverse strand primer

$2 \mu \mathrm{L} \quad 10 \times$ PCR buffer (containing $15 \mathrm{mM} \mathrm{MgCl}_{2}$ )

$0.5 \mu \mathrm{L} \quad$ Taq DNA polymerase

$1 \mu \mathrm{L} \quad$ Nucleic acid (approximately 100 ng cDNA, gDNA or plasmid DNA) $20 \mu \mathrm{L}$ 


\begin{tabular}{|c|c|c|c|}
\hline Initial denaturation & $95^{\circ} \mathrm{C}$ & $10 \mathrm{~min}$ & \\
\hline Denaturation & $95^{\circ} \mathrm{C}$ & $10 \mathrm{~s}$ & \\
\hline Annealing & $60^{\circ} \mathrm{C}$ & $15 \mathrm{~s}$ & x 35 cycles \\
\hline Extension & $72^{\circ} \mathrm{C}$ & $20 \mathrm{~s}$ & \\
\hline Final extension & $72^{\circ} \mathrm{C}$ & $5 \mathrm{~min}$ & \\
\hline Hold & $10^{\circ} \mathrm{C}$ & Hold & \\
\hline
\end{tabular}

\subsubsection{Purification of DNA}

\subsubsection{Gel purification}

Gel purification was used when purified PCR products and plasmid inserts were required for subsequent cloning or sequencing. Bands of interest, separated by gel electrophoresis, were excised from the gel. DNA from these gel pieces was column purified using the Zymoclean ${ }^{\mathrm{TM}}$ Gel DNA Recovery Kit (Zymo Research) following the manufacturer's protocol. DNA eluted was free of gel contaminants and salts that could interfere with future applications.

\subsubsection{DNA purification and concentration}

When high quality DNA was required, DNA Clean and Concentrator kits were used (Zymo Research). Following manufacturer's instructions, the DNA was column purified and concentrated. This purification removed enzymes, unused dNTPs and primers that could interfere with future applications.

\subsubsection{Plasmids and ligation}

The Rapid Ligation kit (Roche) was used when PCR and cDNA fragments were ligated into plasmids for applications such as cloning and biolistic-mediated plant transformations. The plasmid vectors used were the pGEM-T Easy vector system (Promega), pART7 and pART27. Cloning DNA fragments into the vector pGEM-T-Easy required the insert to have an A-tail overhang for easy insertion. Insertion of PCR products into pART7 and pART27 began with preparation of the vector by digesting it with either one or two different restriction endonucleases (Section 2.6.8). The PCR products for cloning into $\mathrm{pART} 7$ and $\mathrm{pART} 27$ were generated using primers containing restriction sites and were gel or column purified (Sections 2.6.5.1 and 2.6.5.2). The insert and vector were quantified spectrophotometrically (Section 
2.6.3.2). The ratio of insert to vector was determined with the formula below and was used in the ligation reaction as outlined below.

$$
\frac{50 \mathrm{ng} \text { vector } \mathrm{x} \boldsymbol{x} \mathrm{kb} \text { of insert }}{\boldsymbol{y} \mathrm{kb} \text { of vector }} \times 3=z \text { ng of insert }
$$

$10 \mu \mathrm{L} \quad 10 \times$ Rapid Ligation buffer

$4 \mu \mathrm{L} \quad 5 \times$ DNA Dilution buffer

$1 \mu \mathrm{L} \quad \mathrm{T} 4$ ligase (Roche)

_ $\mu \mathrm{L} \quad$ Sterile $\mathrm{H}_{2} \mathrm{O}$

_ $\mu \mathrm{L} \quad$ Vector (50 ng)

$\ldots \mathrm{L} \quad$ Insert $(\boldsymbol{z} \mathrm{ng})$

$20 \mu \mathrm{L}$

For PCR fragments with blunt ends (without an A-tail overhang) to be inserted into pGEM-T-Easy, an A-tailing reaction was carried out before ligation, as below.

$0.5 \mu \mathrm{L} \quad 10 \mathrm{mM}$ dATP

$0.5 \mu \mathrm{L} \quad$ Taq DNA polymerase (Roche)

$0.5 \mu \mathrm{L} \quad 10 \mathrm{x}$ buffer

$3.5 \mu \mathrm{L} \quad$ Insert

$5 \mu \mathrm{L}$

The A-tailing reaction was placed in a $70^{\circ} \mathrm{C}$ water bath for $1 \mathrm{~h}$.

\subsubsection{Plasmid miniprep}

Plasmid minipreps were carried out on $3 \mathrm{~mL}$ overnight Escherichia coli and Agrobacterium tumefaciens bacterial cultures using the Axyprep ${ }^{\text {TM }}$ Plasmid Miniprep kit (Axygen Biosciences) according to manufacturer's instructions. Plasmids were extracted from bacterial cells using an alkaline lysis system and column purification. The eluted plasmids are cleaned of cellular lysate and bacterial gDNA. Plasmids extracted from A. tumefaciens were back-transformed into $E$. coli for confirmation of the insert in the vector, due to poor plasmid yield and contamination of the $\mathrm{T}_{\mathrm{i}}$-plasmid when extracting from $A$. tumefaciens cells. 


\subsubsection{Restriction endonuclease reactions}

Restriction endonuclease digests were carried out on plasmid DNA before ligation reactions and for isolation of the plasmid insert. A typical restriction digest is outlined below; the enzyme buffer used was matched to the restriction enzyme used.

$0.5 \mu \mathrm{L} \quad$ Restriction endonuclease enzyme (Roche)

$1 \mu \mathrm{L} \quad 10 \times$ commercial enzyme buffer (Roche)

$1.5 \mu \mathrm{L}$ Plasmid DNA

$7 \mu \mathrm{L} \quad$ Sterile $\mathrm{H}_{2} \mathrm{O}$

$10 \mu \mathrm{L}$

If two enzymes were used in a single reaction, $0.5 \mu \mathrm{L}$ of water was substituted for the second enzyme. The digest reaction was incubated in a $37{ }^{\circ} \mathrm{C}$ water bath for $1.5 \mathrm{~h}$ before the digested DNA was separated by gel-electrophoresis (Section 2.6.3.1).

\subsubsection{DNA sequencing}

Purified DNA of interest was sent to the Waikato University DNA Sequencing facility along with the appropriate primers. DNA was sequenced using BigDye ${ }^{\circledR}$ Terminator v3.1 Cycle Sequencing technology (Applied Biosystems). Analysis of sequenced products was done using Geneious Pro 5.5.6 software (BioMatters Ltd.).

\subsection{Bacterial cloning}

Bacterial cloning was used for sequencing PCR products, as well as for synthesis of constructs used in biolistic- and Agrobacterium-mediated plant transformation experiments.

\subsubsection{General bacterial growth conditions}

The bacterial strains used in this study were Escherichia coli (strain XL1-Blue) and Agrobacterium tumefaciens (strain GV3101). Unless otherwise stated, both bacterial strains were grown on LB-agar or LB-broth (Appendix 1.1.1) with the appropriate antibiotic selection. E. coli and A. tumefaciens cultures were grown at $37^{\circ} \mathrm{C}$ and $28^{\circ} \mathrm{C}$, respectively. Liquid cultures were placed in shaking incubators at $250 \mathrm{rpm}$ and $37^{\circ} \mathrm{C}$ or $28^{\circ} \mathrm{C}$, depending on the bacterial strain. 


\subsubsection{Chemically competent $E$. coli}

E. coli cells were made chemically competent following methods by Inoue et al. (1990).

\subsubsection{Heat-shock transformation of $E$. coli}

Freshly thawed competent $E$. coli cells ( $50 \mu \mathrm{l})$ were incubated on ice with $10 \mu \mathrm{L}$ of the ligation reaction (Section 2.6.6) for $20 \mathrm{~min}$. Cells were then heat-shocked in a $42{ }^{\circ} \mathrm{C}$ water bath for $45 \mathrm{~s}$ before being placed on ice for $5 \mathrm{~min}$. Of this mixture, $20 \mu \mathrm{L}$ was spread on L-agar plate (Appendix 1.1.1) containing antibiotic selection (Appendix 1.3), and blue/white selection chemicals, 5-bromo-4-chloro-3-indolyl galactopyranoside (X-gal, Appendix 1.7.2) and isopropyl- $\beta$-D-thiogalactopyranoside (IPTG, Appendix 1.7.2). Plates were incubated at $37^{\circ} \mathrm{C}$ overnight.

\subsubsection{Electro-competent $A$. tumefaciens}

A. tumefaciens (GV3101) cells were made electro-competent following the methods by McCormac et al. (1998).

\subsubsection{Electroporation transformation of $A$. tumefaciens}

Freshly thawed electro-competent $A$. tumefaciens cells were transformed with pART27 plasmid by electroporation. Agrobacterium cells $(25 \mu \mathrm{L})$ and $1 \mu \mathrm{L}$ of plasmid DNA were pipetted into a pre-chilled electroporation cuvette (Biorad). This cuvette was placed between the electrodes of a Biorad Gene pulser and cells were electroporated at $300 \mathrm{~V}\left(12 \mathrm{kV} \mathrm{cm}^{-1}\right)$. To recover cells, $20 \mu \mathrm{L}$ of solution was pipetted into $1 \mathrm{~mL}$ of LB-broth (Appendix 1.1.1) and placed in a $28^{\circ} \mathrm{C}$ shaking incubator for $2 \mathrm{~h}$ at $250 \mathrm{rpm}$. After incubation, $50 \mu \mathrm{L}$ of LB-broth was spread onto LB-agar plates containing $200 \mathrm{mg} \mathrm{L}^{-1}$ spectinomycin and $200 \mathrm{mg} \mathrm{L}^{-1}$ streptomycin (Appendix 1.3) and placed in a $28^{\circ} \mathrm{C}$ incubator for two days.

\subsection{Plant transformations}

\subsubsection{Biolistic-mediated transformations}

Genes were transiently expressed in plant tissues by using biolistic-mediated transformations. Methods were adapted from those described by Shang et al. (2007). Plant tissues were surface sterilised (Section 2.3.2) and placed on culture tubs containing media \#2 (Appendix 1.1.3). Water was added to $5 \mu \mathrm{g}$ of pART7 constructs to 
make total volume of $10 \mu \mathrm{L}$, which was added to $50 \mu \mathrm{L}$ of water containing $5 \mathrm{mg}$ gold particles ( $1 \mu \mathrm{m}$ diameter, BioRad). To precipitate plasmids onto the gold, $50 \mu \mathrm{L}$ of $2.5 \mathrm{M} \mathrm{CaCl}_{2}$ and $20 \mu \mathrm{L}$ of $100 \mathrm{mM}$ spermidine, were added and the tube was immediately vortexed for $3 \mathrm{~min}$. Gold was pelleted with a brief centrifugation, and $90 \mu \mathrm{L}$ of liquid was pipetted off and discarded. Gold particle bombardment used a helium-driven particle inflow gun (Kiwiscientific Ltd, Levin, NZ). The inflow gun is a bespoke custom made device, based on the particle gun designed by Vain et al. (1993). Tissue on media $\# 2$ was placed at a gun range of $130 \mathrm{~mm}$. Tissue was bombarded with $5 \mu \mathrm{L}$ of gold suspension under $400 \mathrm{kPa}$ of helium with a vacuum of $-90 \mathrm{kPa}$. Tissue was incubated on the same medium at $22{ }^{\circ} \mathrm{C}$ overnight with a $16 \mathrm{~h}$ photoperiod.

\subsubsection{Agrobacterium-mediated transformations}

Agrobacterium-mediated plant transformations followed the methods by Koiwa et al. (2006). The Agrobacterium plate culture (Section 2.7.5) was used to inoculate $3 \mathrm{~mL}$ LB-broth (Appendix 1.1.1) with $25 \mathrm{mg} \mathrm{L}^{-1}$ spectinomycin and $25 \mathrm{mg} \mathrm{L}^{-1}$ streptomycin antibiotic selection (Appendix 1.3). Liquid culture was placed in a shaking incubator (250 rpm) overnight at $28^{\circ} \mathrm{C}$. Of this broth, $2.5 \mathrm{~mL}$ was transferred to $250 \mathrm{~mL}$ fresh LB-broth with selection and placed in the shaking incubator for $24 \mathrm{~h}$. The culture was spun down at $6 \mathrm{~K}$ for $5 \mathrm{~min}$ in a SLA-1500 rotor (Thermo Scientific). The supernatant was discarded and the pellet resuspended in the Agrobacterium transfection solution (Appendix 1.2). The transfection solution was then used to dip inflorescences on Arabidopsis thaliana plants grown in the greenhouse (Section 2.3.1). Inflorescences were held in solution for $30 \mathrm{~s}$ before plants were placed overnight inside a black plastic bag pre-moistened with water. The following day, plants were transferred back to the greenhouse.

\subsection{General pigment analyses}

\subsubsection{Betalain pigment extraction}

Betalain pigments from fresh or freeze dried tissues were extracted in $2 \mathrm{~mL}$ of betalain XT buffer (Appendix 1.6.1). Tissues in extraction buffer were placed at $4{ }^{\circ} \mathrm{C}$ for $2 \mathrm{~h}$, centrifuged and the extract pipetted into new tube. Extraction of betalain pigments in fresh XT buffer was repeated over $24 \mathrm{~h}$ or until the majority pigments had been removed. All extracts from individual samples were pooled. 


\subsubsection{Betalain alkaline test}

An alkaline test was used to determine whether a solution contained anthocyanins or betacyanins followed the methods by Nielson and Harley (1996). Equal amounts of water, $4 \mathrm{M} \mathrm{NaOH}$ and samples were added to $1.5 \mathrm{~mL}$ microcentrifuge tubes. Solutions were examined for a colour change of purple/red to blue/green to indicate anthocyanins, or yellow to indicate betacyanins. 


\section{Chapter 3: Betalain localisation}

\subsection{Introduction}

Betalain pigments are often described as the anthocyanin replacement for many plant species in the order Caryophyllales. This replacement hypothesis is largely based on the visual similarities between the two pigment types. Both anthocyanins and betalains are stored in the vacuole and are often similar in colour (Tanaka et al., 2008). In addition, up-regulation of these pigments occurs in response to similar environmental factors (Stafford, 1994) and at similar developmental stages (Lee \& Collins, 2001). Although betalains replacing anthocyanins in plants outwardly appears true, little research has been conducted to test this hypothesis. One area that needs to be compared is the localisation of the two pigments. If betalains functionally replace anthocyanins, the pigments should be found in similar tissues.

Not much is known about the localisation of betalains. The few published examples describe betalains in: the foliar epidermis, palisade and spongy mesophyll tissues (Lee \& Collins, 2001); the hypodermis and collenchyma in several cacti (Mosco, 2012); vascular tissues in roots and hypocotyls of red beet (Beta vulgaris subsp. vulgaris var. conditiva); and the epidermis and bundle sheath cells in petioles (Petrus-Vancea et al., 2010). Further information on betalain distribution in different organs is required to compare with the extensive published information on anthocyanin localisation. Published examples of tissue-specific anthocyanin localisation are summarised in Table 1.1. It is presently unknown if betalains occur in all comparable locations.

\subsubsection{Aim}

This chapter addresses the questions of whether betalains occur within the same or different tissues as anthocyanins, and if the distribution of betalains is similar in different betalain-producing plants. In addition, analyses were made of whether the two types of betalain compounds, betaxanthin and betacyanins, always co-occur. 


\subsection{Materials and Methods}

\subsubsection{Plant sources}

A range of betalain-producing plants were analysed in this study, including two native New Zealand species (Figure 3.1). Plants were selected to have representatives from as many different families the Caryophyllales as possible, but were limited to plant species readily available. Plants were: (1) grown from seed - vegetable amaranth (Amaranthus tricolor), red orach (Atriplex hortensis), leafy goosefoot (Chenopodium foliosum), Swiss chard (Beta vulgaris subsp. cicla cv. 'Bright Lights'), beetroot (Beta vulgaris subsp. vulgaris cv. 'Red Globe') and spinach (Spinacia oleracea); (2) purchased as mature plants - living stone (Lithops aucampiae euniceae), bougainvillea (Bougainvillea spectabilis) and ruby rainbow cactus (Echinocereus rigidissimus rubispinus); (3) sourced from the plants growing in the local environment - native ice plant (Disphyma australe) and New Zealand spinach (Tetragonia tetragoniodes) from Wellington south coast; or (4) donated by Ranjith Pathirana from Plant \& Food Research, Palmerston North - ulluco (Ullucus tuberosus). For plant growth conditions see Section 2.3.1.

\subsubsection{Sectioning}

To map where pigments occur within these species, fresh hand-sections were taken through multiple organs (primary root, hypocotyl, stem internode, petiole and fully expanded lamina) from three individuals of each species. Sections were photographed using the Olympus Provis AX70 compound microscope (Section 2.4.2).

The following plants were sectioned once their first true leaf had fully expanded: vegetable amaranth, red orach and leafy goosefoot. Sectioning of Swiss chard, spinach, ice plant, New Zealand spinach and beetroot was carried out on plants that had their first fully expanded leaves and on mature plants at an unknown age. The remaining plants, ulluco, bougainvillea, ruby rainbow cactus and living stone were all mature plants of an unknown age when sectioned.

\subsubsection{In situ absorptance spectra}

Individual cell vacuole absorptances were obtained from sections of purple, yellow and white six month old Swiss chard plants. Three of each colour plant were chosen with 
visually similar pigmentation and were sectioned for five organs; root, hypocotyl, stem, young leaf and old leaf. The young leaf and the old leaf were defined as the youngest unexpanded leaf and the youngest fully expanded leaf, respectively. All sections were mounded on glass slides in water. Profiles of visible light absorptance (400 - $700 \mathrm{~nm})$ were taken at $20 \mathrm{~nm}$ wavelength intervals for individual cell vacuoles using an Ocean Optics (MO, USA) USB4000 spectrometer, connected via a fibre optic cable to an eyepiece of the compound microscope. Incident white light used for the readings was provided by the microscope. The remaining tissue of each organ from the purple, yellow and white plants was immediately placed into the $-80{ }^{\circ} \mathrm{C}$ freezer for high-performance liquid chromatography (HPLC) analyses.

\subsubsection{Tissue preparation for HPLC}

Samples (described in Section 3.2.3) were ground to powder in liquid nitrogen before freeze drying in a Labconco freeze dry system (model \#79480, Kansas City, MO) for $48 \mathrm{~h}$. Ground freeze dried samples were weighed out to $50 \mathrm{mg}$ and transferred into $2 \mathrm{~mL}$ microcentrifuge tubes. To each tube, $1 \mathrm{~mL}$ betalain XT buffer (Appendix 1.6.1) was added. Multiple extractions using betalain XT buffer were carried out over $48 \mathrm{~h}$ at $4{ }^{\circ} \mathrm{C}$ to remove the majority of pigment. Extracts were combined into a single tube and reduced under vacuum to a final volume of approximately $0.4 \mathrm{~mL}$. Samples were cooled in liquid nitrogen for $30 \mathrm{~min}$ before freeze drying for a second time overnight. Freeze dried extracts were reconstituted with distilled $\mathrm{H}_{2} \mathrm{O}$ to $0.5 \mathrm{~mL}$, or $1 \mathrm{~mL}$ for the darker pigment colours. Reconstituted extracts were vortexed and filtered through a $0.4 \mu \mathrm{m}$ pore regenerated cellulose membrane filter into a new microcentrifuge tube. Sample volumes were doubled to a final volume of either $1 \mathrm{~mL}$ or $2 \mathrm{~mL}$, depending on the initial reconstitution.

\subsubsection{HPLC analysis}

Aliquots $(300 \mu \mathrm{L})$ of the extracts prepared in Section 3.2.4 were analysed using a Dionex UltiMate 3000 HPLC delivery system with a Dionex 3000 Diode Array Detector (DAD). Methods for HPLC were following those of Kugler et al. (2004, 2007) and Svenson et al. (2008) with minor modifications. Separation of compounds began with a mobile phase of $1 \%(\mathrm{v} / \mathrm{v})$ formic acid in water (solvent $\mathrm{A}$ ), followed by $80 \%$ (v/v) acetonitrile in water (solvent B), through a Phenomenex $150 \times 3 \mathrm{~mm}$ i.d. Synergi hydrocolumn fitted with a Phenomenex C18 4 x 3 mm guard column. The column was 
kept at a constant temperature of $25^{\circ} \mathrm{C}$ with a flow rate of $500 \mu \mathrm{L}$. The betaxanthin gradient (monitored at $470 \mathrm{~nm}$ ) began with $100 \%$ A, decreasing to $80 \%$ A at $62 \mathrm{~min}$, and then a linear gradient to $100 \% \mathrm{~B}$ at $67 \mathrm{~min}$, remaining at $100 \% \mathrm{~B}$ for a further $10 \mathrm{~min}$. The betacyanin gradient (monitored at $538 \mathrm{~nm}$ ) started with $98 \%$ solvent A which dropped to $67 \% \mathrm{~A}$ at $32.5 \mathrm{~min}$, followed by $98 \% \mathrm{~B}$ at $35 \mathrm{~min}$, and remaining at $98 \%$ B for a further 1.5 min. HPLC traces were processed with Dionex Chromeleon 6.8 (SR10).

\subsubsection{Tissue preparation for Liquid Chromatography-Mass Spectrometry}

\section{(LC-MS)}

LC-MS analyses were performed on mature (6 months old) purple, red, orange and yellow Swiss chard plants. Whole plants were freeze dried and ground to powder. Samples were then analysed by Nigel Joyce at Plant \& Food Research. For each sample $20 \mathrm{mg}$ of tissue was extracted in $1 \mathrm{~mL}$ of $50 \%$ water: $50 \%(85 \%(\mathrm{v} / \mathrm{v})$ methanol: $15 \%$ (v/v) acetic acid) by vortex mixing, standing overnight at $4{ }^{\circ} \mathrm{C}$ and additional vortex mixing. The solvent extract was then centrifuged and filtered via a $0.22 \mu \mathrm{m}$ nylon filter into amber glass vials.

\subsubsection{LC-MS analyses}

The LC-MS system consisted of a Thermo Electron Corporation (San Jose, CA, USA) Finnigan Surveyor MS pump, Thermo Accela Open Auto sampler (PAL HTC-xt with DLW), Finnigan Surveyor PDA plus detector and a ThermaSphere TS-130 column heater (Phenomenex, Torrance, CA, USA). A $2 \mu \mathrm{L}$ aliquot of each prepared extract was separated with a mobile phase consisting of $1 \%(\mathrm{v} / \mathrm{v})$ formic acid in water $(\mathrm{A})$ and acetonitrile:water, 80:20 (B) by reverse phase chromatography (Aqua guard cartridge $4 \times 2 \mathrm{~mm}, 10 \mu$ and Synergi-Hydro RP C18, $4 \mu, 80 \AA$, 250 x $2.1 \mathrm{~mm}$, Phenomenex, Torrance, CA, USA) maintained at $25^{\circ} \mathrm{C}$ with a flow rate of $200 \mu \mathrm{L} \mathrm{min}{ }^{-1}$. The first gradient that was applied monitored betaxanthins at $470 \mathrm{~nm}$ : $1-2 \mathrm{~min} 100 \%$ (A), 2 - 62 min linear gradient to $80 \%$ (A), 62 - 67 min linear gradient to $0 \%$ (A), regenerated back to $100 \%$ (A). The second gradient monitored betacyanins at $538 \mathrm{~nm}$ : 1 - 30 min linear gradient from $100 \%$ to $67 \%$ (A), regenerated back to $100 \%$ (A).

The eluent was scanned by PDA (200 - $700 \mathrm{~nm}$ ) and API-MS (LTQ, 2D linear ion-trap, Thermo-Finnigan, San Jose, CA, USA) with electrospray ionisation (ESI, positive). 
Data were acquired for parent masses from m/z 250 - 1500 Da with $\mathrm{MS}^{4}$ fragmentation. Data were processed with the aid of Xcalibar ${ }^{\circledR 2.1 ~(T h e r m o ~ E l e c t r o n ~}$ Corporation).

\subsubsection{Photometric quantification and identification of betalains}

Quantification of betaxanthins and betacyanins followed similar methods to Kugler et al. (2004). Extracts of samples described in Section 3.2.4 were diluted in the McIlvaine buffer system (Appendix 1.6.2) for absorbance maxima between $0.1 \leq A \leq 0.2$ for the wavelengths $470 \mathrm{~nm}, 538 \mathrm{~nm}, 650 \mathrm{~nm}$. All measurements were performed in duplicate using a Jasco V-530 UV/VIS spectrophotometer. Total betalain content (BC) was calculated with the equation adapted from Cai and Corke (1999):

$$
\mathrm{BC}\left(\mathrm{mg} \mathrm{L}^{-1}\right)=A \times \mathrm{DF} \times \mathrm{MW} \times \frac{1000}{\epsilon} \times L
$$

Where $A$ is the absorbance value at the absorbance maximum $\left(\lambda_{\max }\right)$, DF is the dilution factor, and $L$ is the path length of the cuvette $(1 \mathrm{~cm})$. Betacyanins were calculated based on betanin parameters: molar extinction coefficient $(\epsilon)=60000 \mathrm{~L} / \mathrm{mol} \cdot \mathrm{cm}$ in $\mathrm{H}_{2} \mathrm{O}$, MW $=550, \lambda_{\max }=538 \mathrm{~nm}$. Betaxanthins were calculated based on dopaxanthin parameters: $\epsilon=48000 \mathrm{~L} / \mathrm{mol} \cdot \mathrm{cm}$ in $\mathrm{H}_{2} \mathrm{O}, \mathrm{MW}=390, \lambda_{\max }=470 \mathrm{~nm}$.

Identification of betalainic compounds from the HPLC and LC-MS analyses were based upon published compound fragment information (Herbach et al., 2005; Kugler et al., 2004, 2007; Nemzer et al., 2011; Svenson et al., 2008), with the aid of the review by Strack et al. (2003). Compounds lower than $0.005 \mathrm{mg} \mathrm{g}^{-1} \mathrm{DW}$ were excluded from further analyses, compounds less than $0.025 \mathrm{mg} \mathrm{g}^{-1} \mathrm{DW}$ were recorded as trace. 

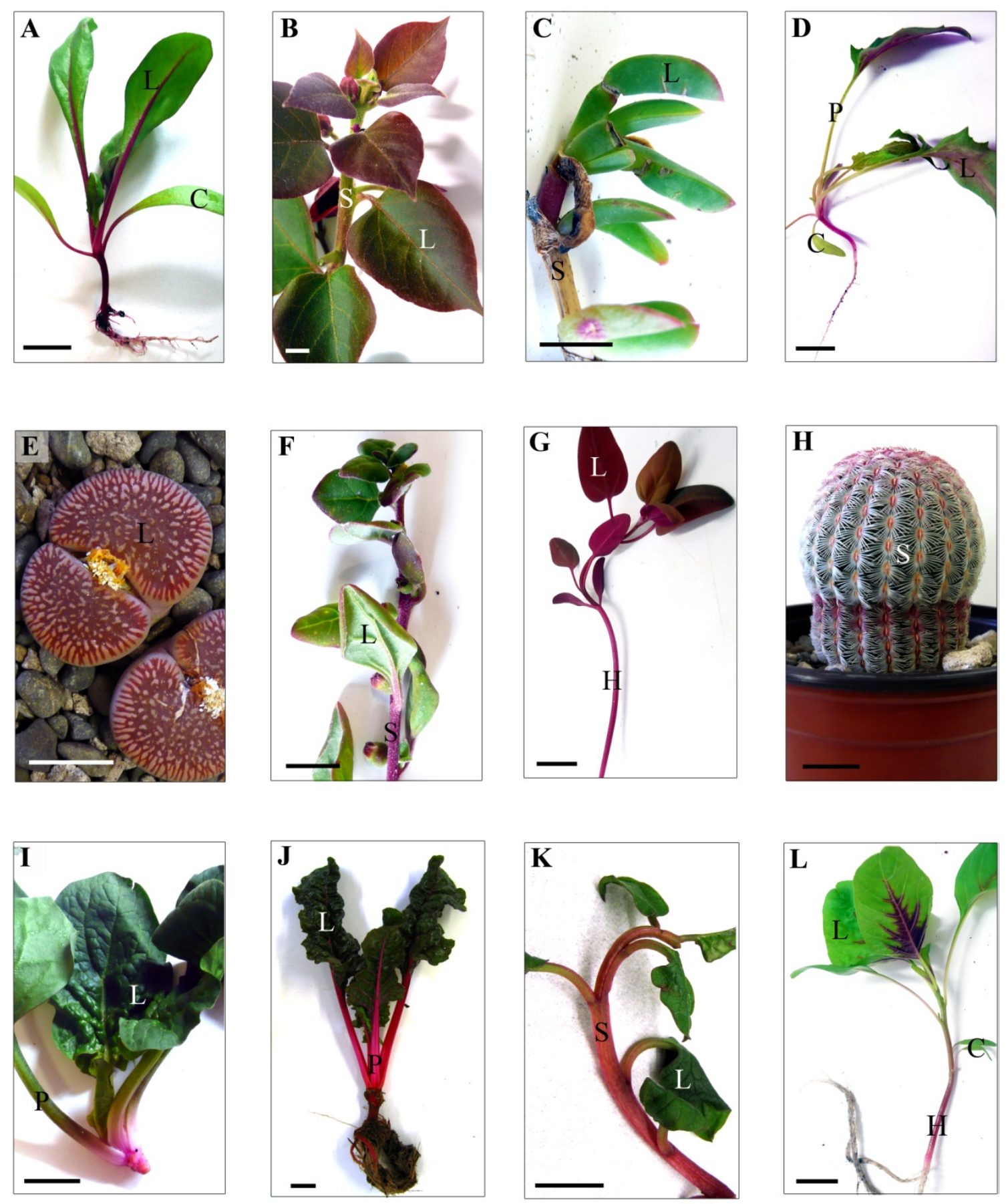

Figure 3.1 Examples of each betalain-producing plant species sectioned. (A) Beetroot; (B) Bougainvillea; (C) Ice plant; (D) Leafy goosefoot; (E) Living stone; (F) New Zealand spinach; (G) Red orach; (H) Ruby rainbow cactus; (I) Spinach; (J) Swiss chard; (K) Ulluco; (L) Vegetable amaranth. Key: C cotyledon, H hypocotyl, L leaf, P petiole, S stem. Scale bars: $\mathbf{F}=20 \mathrm{~mm}$, all others $=10 \mathrm{~mm}$. 


\subsection{Results}

\subsubsection{Localisation of betalains}

The histological locations of betalains in 12 species are summarised in Table 3.1. Ten out of 12 species produced betalains within multiple organs; the exceptions being the living stone and ruby rainbow cactus plants, for which betalain pigmentation was visible only in their leaves or spines, respectively. Ruby rainbow cactus was excluded from further betalain localisation comparisons as the morphology of the cactus made cross-species comparisons difficult. Betalains were most often present in the lamina (present in ten out of 11 species) and petiole (present in eight out of 11 species) of leaves, as well as in the stem (present in ten out of 11 species). Betalain pigmentation was also common in the hypocotyl (present in six out of 11 species) and root (present in five out of 11 species) organs. Although the number of pigmented cells differed notably between species, the pattern of pigmented tissue types within these organs appeared similar.

The body of a vascular plant, as described by Esau (1965), is composed of three tissues systems, the dermal, the vascular, and the ground (or fundamental) tissues. All three system types contained betalains within multiple organs as found in this study. The tissues with observed pigmentation were grouped as follows: dermal tissues - the epidermis, trichomes, and bladder cells; ground tissues - spongy and palisade mesophyll, collenchyma, cortex cells, pith, and endodermis; vascular tissues - phloem parenchyma, bundle sheath cells, storage parenchyma and pericycle. In the leaves, pigmentation was prominent dermal and ground tissues of the lamina for ten out of 11 species (Figures 3.2 and 3.3). Dermal pigmentation was also the most commonly pigmented tissue type in the petiole, present in eight out of 11 species (Figure 3.4). In the lamina and petiole, betalain pigments were also commonly observed within vascular tissues. The most commonly pigmented cell types within the stem (Figures 3.5 and 3.6), hypocotyl (Figure 3.7), and root (Figure 3.8), were the dermal and ground tissue types. Vascular cell types were also commonly pigmented in the stem, hypocotyl and root organs. 


\subsubsection{Other pigmented structures}

During histological analyses, several other pigmented structures were observed. Epidermal bladder cells containing betacyanins were observed in two species, New Zealand spinach stems and leaves (Figure 3.9A) and ulluco leaves (Figure 3.9B). Cacti spines were pigmented for ruby rainbow cactus (Figure 3.9C). Pigmented trichomes were observed on bougainvillea (Figure 3.9D). In the leaves of living stone, yellow-orange bodies were seen in sub-epidermal layers (Figure 3.9E). Under blue light these bodies autofluoresced green, a characteristic of betaxanthins (Figure 3.9F). Upon closer inspection under confocal microscopy, these autofluorescent bodies were determined to be plastids. Identification is based on their co-localisation with red autofluorescence, typical of chlorophyll (Figure 3.10). Also, carotenoids are known to autofluoresce green under blue light (Gillbro \& Cogdell, 1989).

\subsubsection{Differential localisation of betaxanthins and betacyanins}

For some species, betacyanins and betaxanthins appeared to accumulate in different tissues. In bougainvillea, betacyanins were found in the stem (Figure 3.11A) and leaves (Figure 3.2B), but only betaxanthins were observed in the roots (Figure 3.11B). Separation of betalain compounds was also noted in ulluco. Ulluco leaves appeared to be pigmented mostly with betacyanins (Figure 3.11C), whereas the stem apparently contained both pigment types (Figure 3.11D) and the roots contained mostly betaxanthins (Figure 3.11E). Furthermore, neighbouring tissue types in ulluco stems contained different compounds. Betaxanthins were observed in the endodermis while betacyanins were found in the cortical cells, and primary tissues of the developing lateral root (Figure 3.11D). In the stems and hypocotyls of purple, red and orange Swiss chard, neighbouring cells had varying concentrations of betalain compounds (Figure 3.11F). In situ absorptance analyses of these tissues detected maximum absorptance peaks for betaxanthins $(470 \mathrm{~nm}$ ) versus betacyanins $(538 \mathrm{~nm}$ ) (Figure $3.11 \mathrm{G})$. The ratio of betaxanthins and betacyanins correlated with the colour observed in these cells, and indicated that these compounds were located together in some cells and separately in others. 


\subsubsection{Pigment analyses of Swiss chard}

\subsubsection{In situ absorptance analyses}

In situ absorptance analyses were performed on sections of purple, yellow and white Swiss chard in an attempt to correlate colours observed with pigment type. In situ absorptance graphs and sections confirmed the visual the pigmentation differences seen between leaves and stems (Figure 3.12). Cell vacuoles of white (Figure 3.12A, 3.12B, and 3.12C) and yellow plants (Figure 3.12D, 3.12E and 3.12F) were found to contain only betacyanins (peak at $538 \mathrm{~nm}$ ) or betaxanthins (peak at $470 \mathrm{~nm}$ ) respectively, and only differed in concentration between the two organs. For purple plants (Figure 3.12G, $3.12 \mathrm{H}$ and 3.12I), cell vacuoles found in the stem frequently contained equal amounts of betaxanthins and betacyanins. In contrast, petioles only contained betacyanins.

\subsubsection{HPLC and LC-MS analyses}

HPLC analyses confirmed presence/absence of betacyanins and betaxanthins within five organs of white, yellow and purple Swiss chard (representative HPLC chromatograms are in Figures 3.13, 3.14, and 3.15). No betalains were detected in the white Swiss chard tissues. Betalains must have been below trace amounts (less than $0.005 \mathrm{mg} \mathrm{g}^{-1} \mathrm{DW}$ ), as pigment was seen in the in situ absorptance analyses (Figure 3.12). This is likely due to the HPLC extraction methods which used whole leaves, whereas, the purple pigmentation observed in these leaves was only at the base of the petioles. The ratios of betacyanins versus betaxanthins found in the purple and yellow samples are represented in Figure 3.16. For both the purple (Figure 3.16A) and yellow (Figure 3.16B) plants the pigment concentration was the highest in the leaves than the other organs. In the purple plants, betaxanthin pigments were not present in the leaves only in the stems, hypocotyls and the roots. In those organs, betaxanthins accounted for approximately a third of the total betalains in each organ. In the HPLC traces for yellow plants, a trace amount of betacyanin was found in all organs. The precursor betalamic acid was detected in purple and yellow Swiss chard organs that also contained betaxanthins (Figure 3.17), but not in organs containing betacyanins only (i.e. purple leaves). Absolute values of betalamic acid in these tissues could not be quantified, as the absorbance maximum for betalamic acid $\left(\lambda_{\max }=430 \mathrm{~nm}\right)$ was not measured for sample extracts on the spectrophotometer. 
LC-MS analyses supported the compound identification from the HPLC results. Interpretation of LC-MS data (Figure 3.18) is presented in Table 3.2. These analyses had similar trends and pigment identity to HPLC analyses for purple and yellow plants, but differed in concentration of the compounds slightly. Red and orange had similar LC-MS profiles to each other, containing similar levels of betacyanin and betaxanthin compounds. The observed difference in colour between red and orange plants was likely that red plants had higher levels of total betalain content than the orange. Compound structures of betalains identified through HPLC and LC-MS are in Figures 3.19, 3.20, and 3.21 .

Betalain identities from purple Swiss chard are listed in Table 3.3. The three major betalainic compounds within purple plants were betanin, betanidin and glutamine-bx. Levels of betalainic compounds differed slightly between different organs. Stem, hypocotyl, and root tissues contained similar compounds, but differed in absolute pigment concentration. The HPLC profiles of leaves differed to the other organs. The leaves contained betanin, isobetanin, lampranthin II and isolampranthin II, but not the aglycone, betanidin. Whereas, lampranthin II and isolampranthin II were only present in the leaves, not in the stems, hypocotyl or roots. Compound ratio changes between betanin and isobetanin was also detected between organs. In addition to greater amounts of isobetanin in the leaves than other organs, the older leaves had higher levels of isobetanin versus betanin when compared to the young leaves.

Compound identities in yellow Swiss chard are listed in Table 3.4. The major pigments in all of the organs were glutamine- and dopamine-bx with most other compounds in minor or trace amounts. Like the purple HPLC results, there were small differences in betalainic compounds between yellow organs. The most notable change was the presence of 17- and 15-decarboxy betanidin in leaves versus the presence of betanin in the root and hypocotyls, with the stem containing traces of both compounds. Glycine-bx, glutamate-bx and proline-bx were all more common in the leaves than the other organs. Trace amounts of 3-methoxytyramine-bx and $\gamma$-aminobutyric acid-bx were found in the old leaf and young leaf respectively. 
Table 3.1 Location of betalains observed in sections of different plant organs.

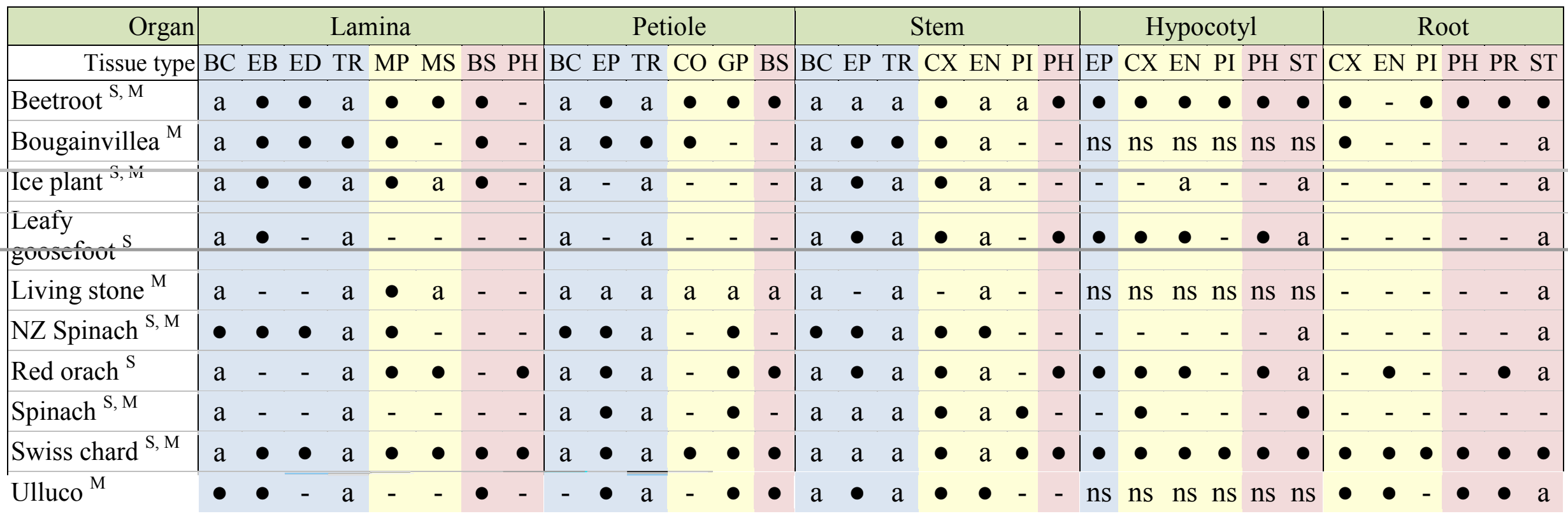




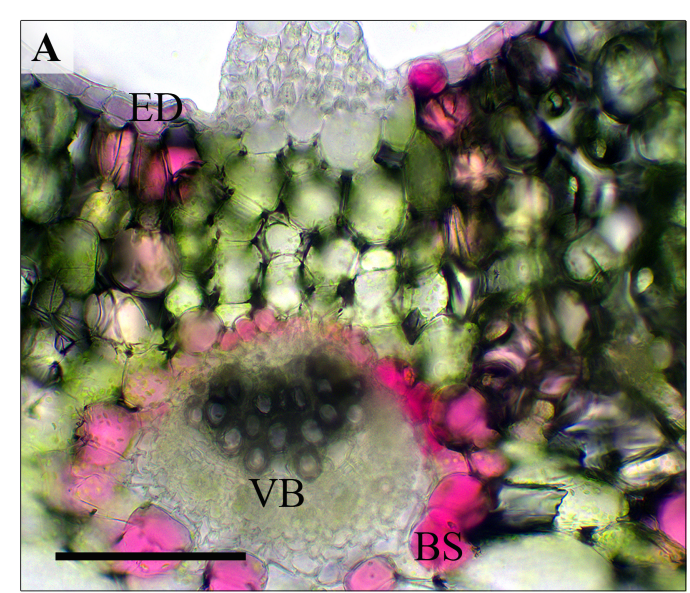

D

$\vec{\perp}$
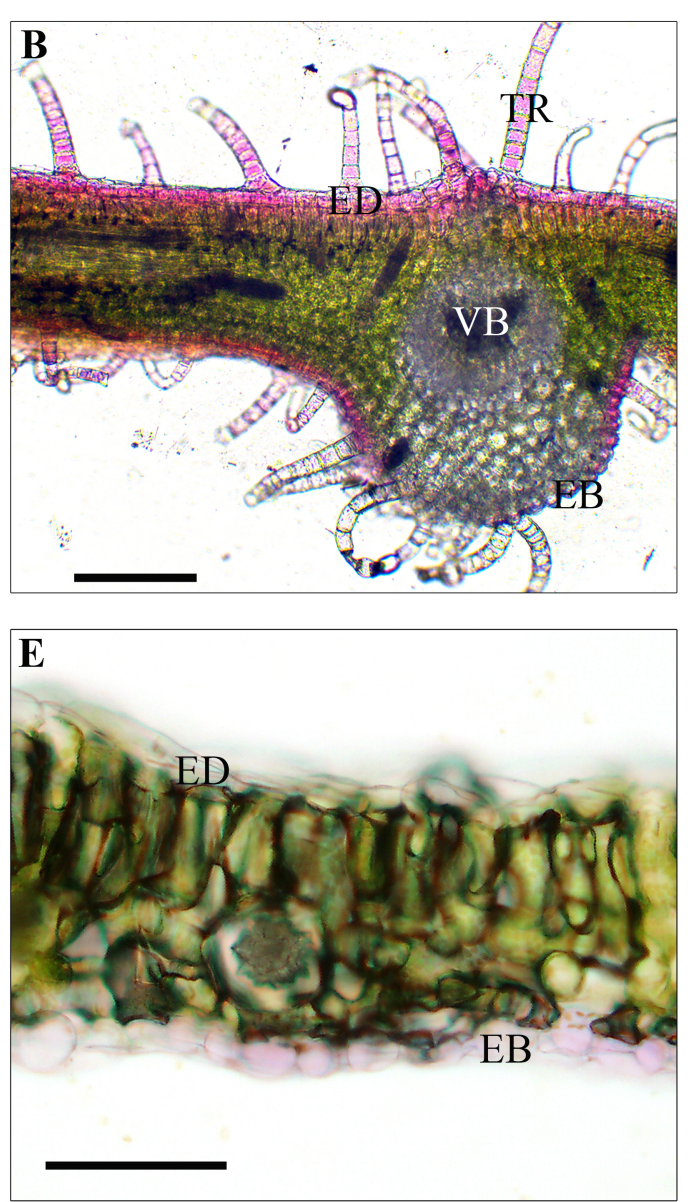
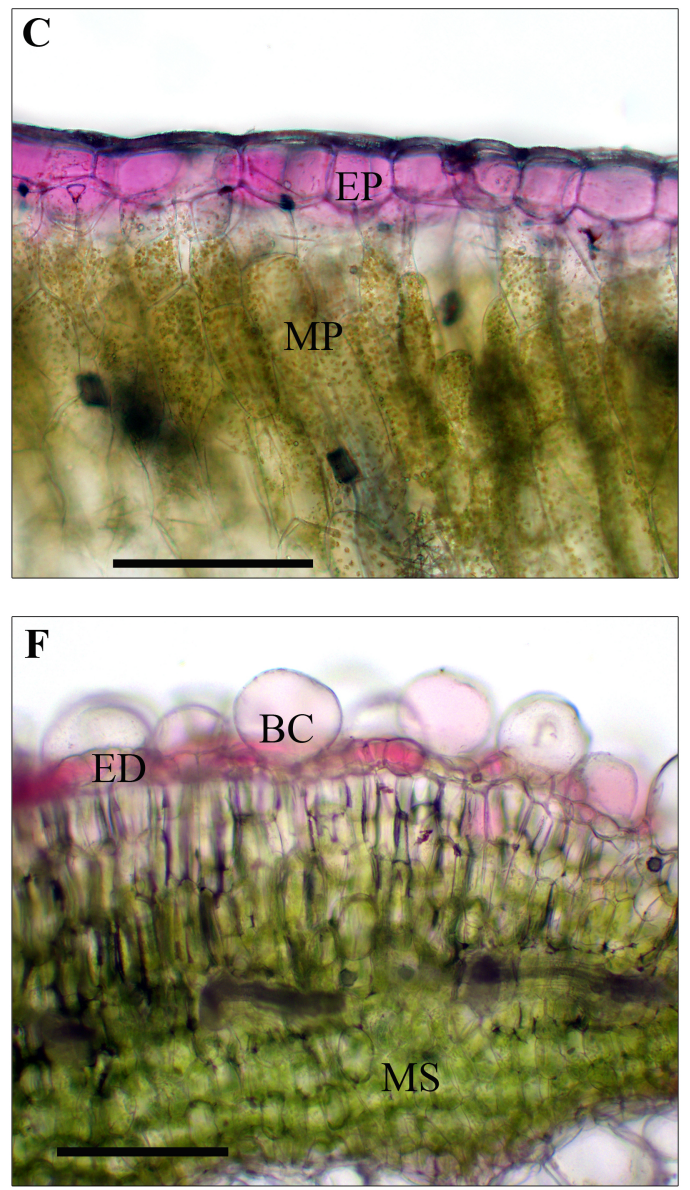

Figure 3.2 Examples of betalain-containing lamina sections for each species. All are transverse sections and mounted in water. (A) Beetroot; (B) Bougainvillea; (C) Ice plant; (D) Leafy goosefoot; (E) Living stone; (F) NZ spinach. Key: BC bladder cell, BS bundle sheath, EB abaxial epidermis, ED adaxial epidermis, EP epidermis, MP palisade mesophyll, TR trichome, VB vascular bundle. Scale bars: $\mathbf{A}=40 \mu \mathrm{m} ; \mathbf{B}=250 \mu \mathrm{m}$; $\mathbf{C}=150 \mu \mathrm{m} \mathbf{D}=100 \mu \mathrm{m} ; \mathbf{E}=50 \mu \mathrm{m} ; \mathbf{F}=300 \mu \mathrm{m}$. All sections are unstained. 

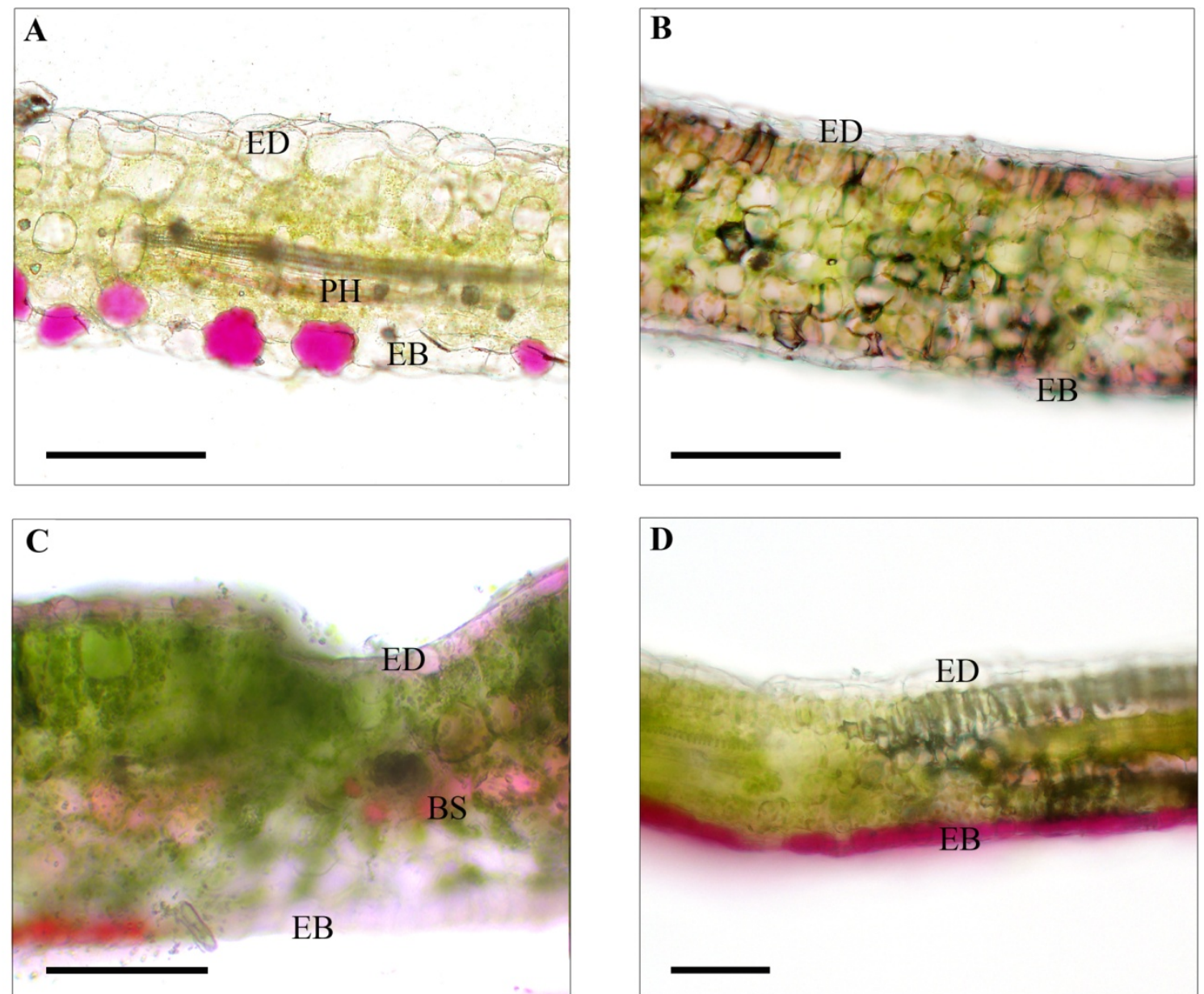

Figure 3.3 Examples of betalain-containing lamina sections for each species (continued). All are transverse sections and mounted in water. (A) Red orach; (B) Swiss chard; (C) Ulluco; (D) Vegetable amaranth. Scale bars: A $=250 \mu \mathrm{m} ; \mathbf{B}=150 \mu \mathrm{m} ; \mathbf{C}=250 \mu \mathrm{m} ; \mathbf{D}=100 \mu \mathrm{m}$. All sections are unstained. 

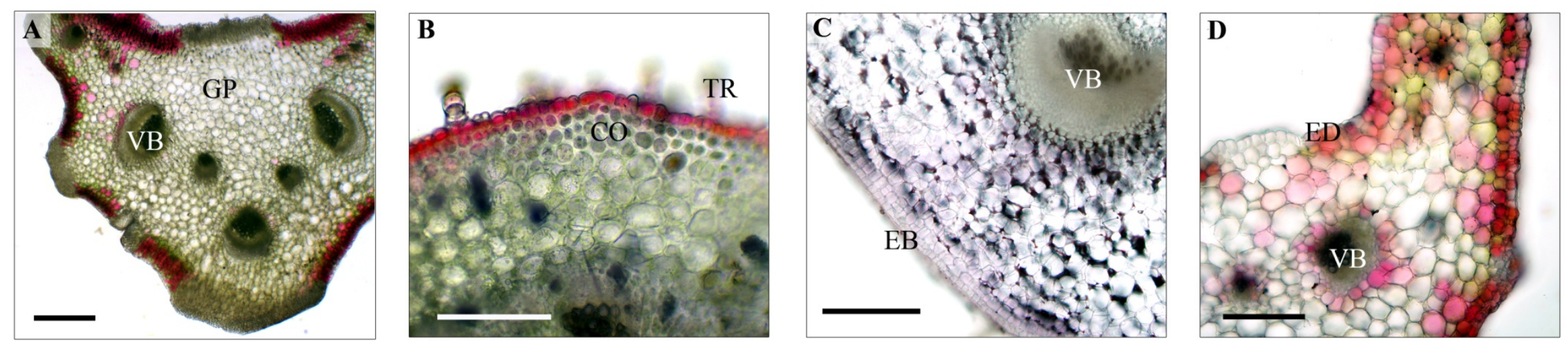

$\mathbf{E}$
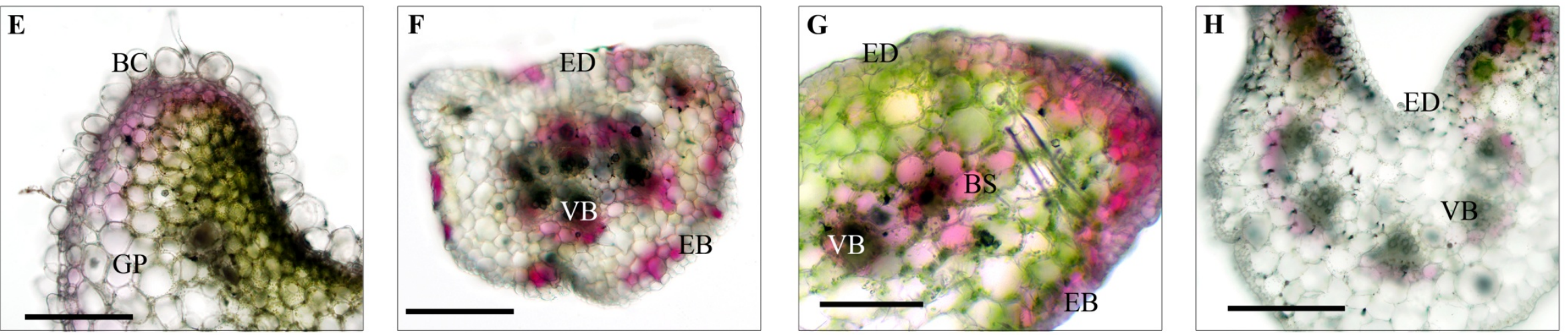

Figure 3.4 Examples of betalain-containing petiole sections for each species. All are transverse sections and are mounted in water. (A) Beetroot; (B) Bougainvillea; (C) NZ spinach; (D) Red orach; (E) Spinach; (F) Swiss chard; (G) Ulluco; (H) Vegetable amaranth. Key: BC bladder cell, BS bundle sheath, CO collenchyma, EB abaxial epidermis, ED adaxial epidermis, GP ground parenchyma, TR trichome, VB vascular bundle, Scale bars $=250 \mu \mathrm{m}$. All sections are unstained. 

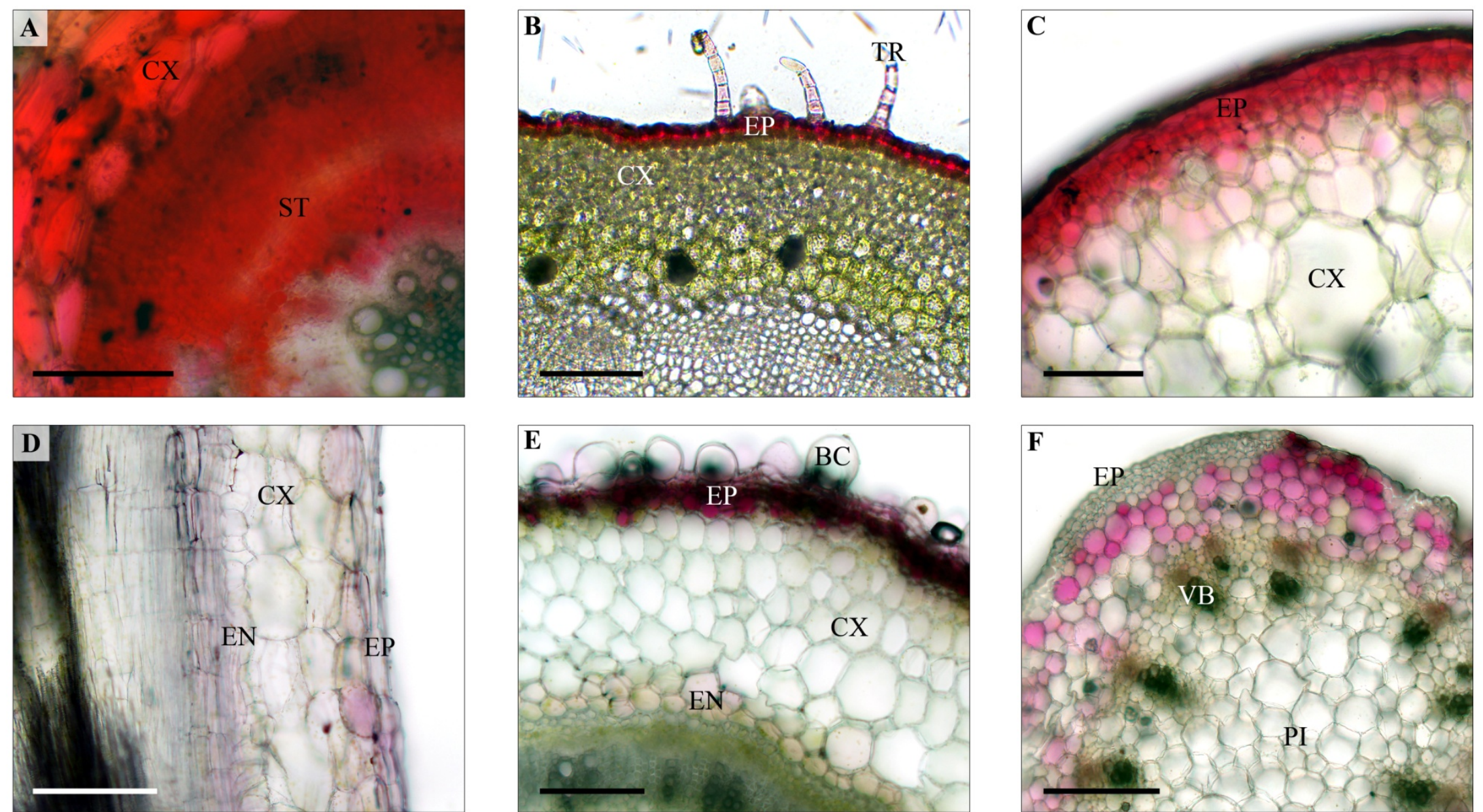

Figure 3.5 Examples of betalain-containing stem sections for each species. All are transverse sections except for D which is a longitudinal section and all are mounted in water. (A) Beetroot; (B) Bougainvillea; (C) Ice plant; (D) Leafy goosefoot; (E) NZ spinach; (F) Red orach. Key: BC Bladder cell, CX cortex, EN endodermis, EP epidermis, PI pith, ST storage parenchyma, TR trichome, VB vascular bundle. Scale bars $=200$ $\mu \mathrm{m}$. All sections are unstained. 

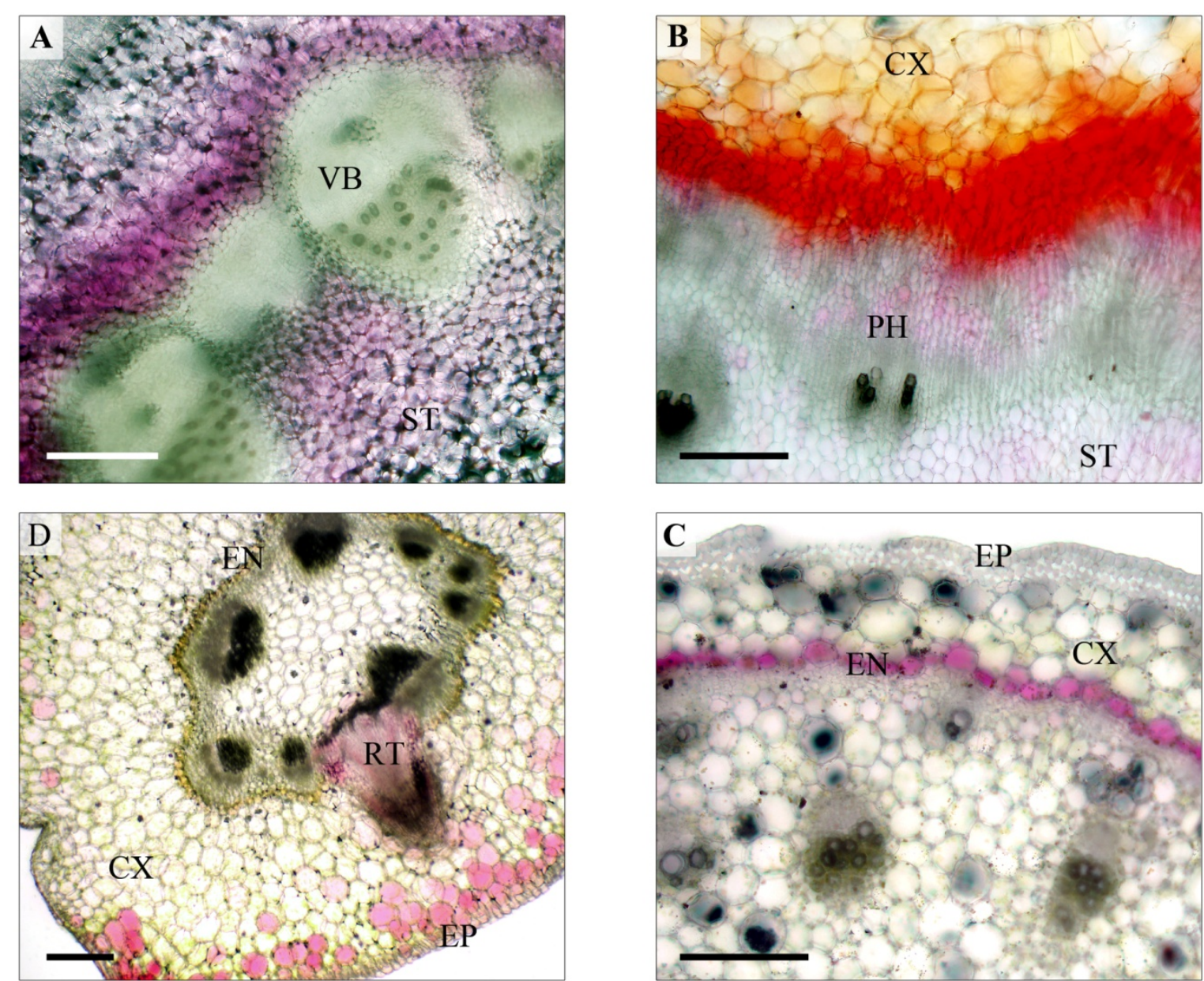

Figure 3.6 Examples of betalain-containing stem sections for each species (continued). All are transverse sections and are mounted in water. (A) Spinach; (B) Swiss chard; (C) Ulluco; (D) Vegetable amaranth. Key: CX cortex, EN endodermis, EP epidermis, PI pith, PH phloem parenchyma RT emerging lateral root, ST storage parenchyma TR trichome, VB vascular bundle. Scale bars $=200 \mu \mathrm{m}$. All sections are unstained. 

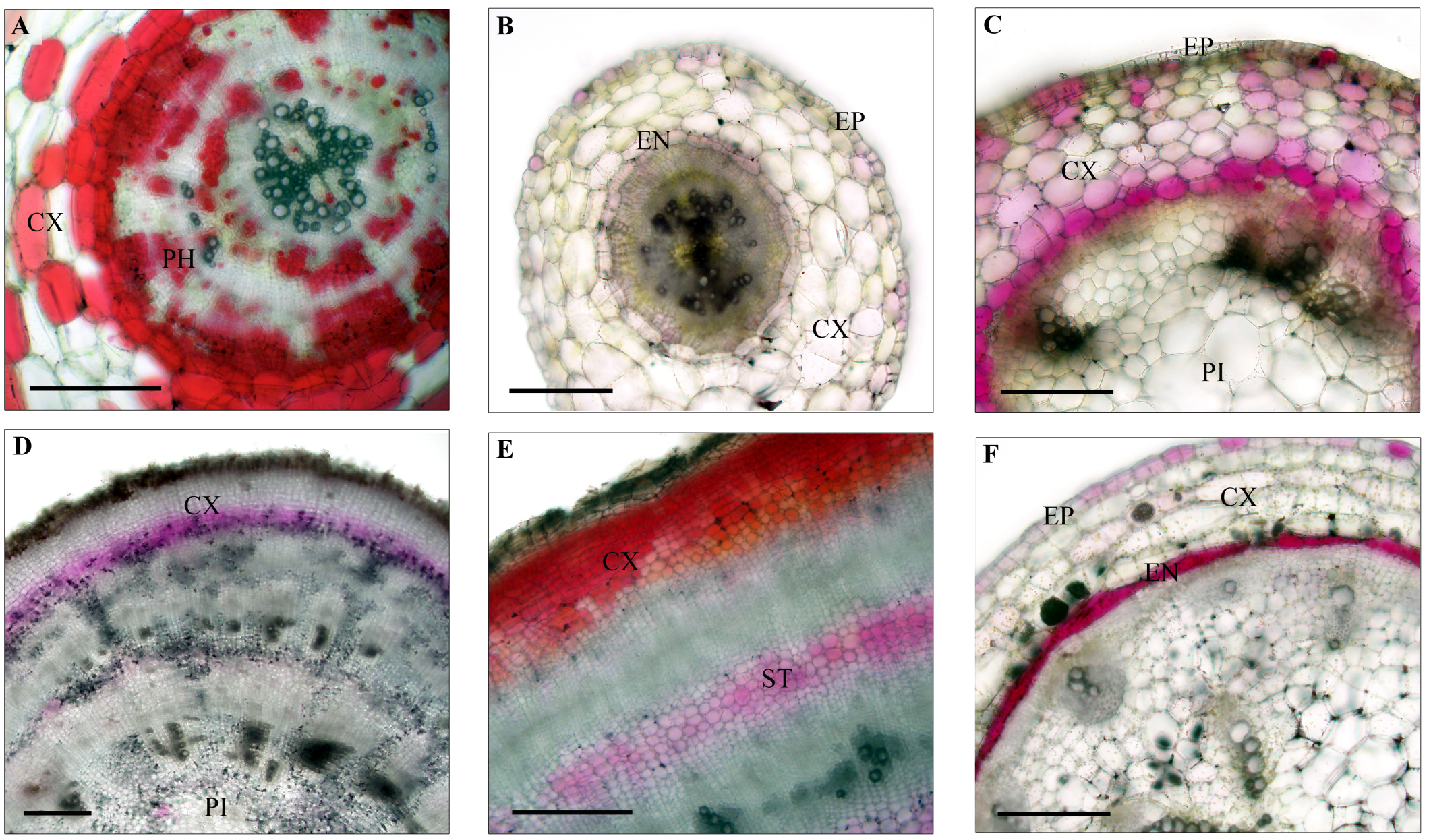

Figure 3.7 Examples of betalain-containing hypocotyl sections for each species. All are transverse sections and are mounted in water. (A) Beetroot; (B) Leafy goosefoot; (C) Red orach; (D) Spinach; (E) Swiss chard; (F) Vegetable amaranth. Key: CX cortex, EN endodermis, EP epidermis, PH phloem parenchyma, PI pith, ST storage parenchyma. Scale bars $=200 \mu \mathrm{m}$. All sections are unstained. 

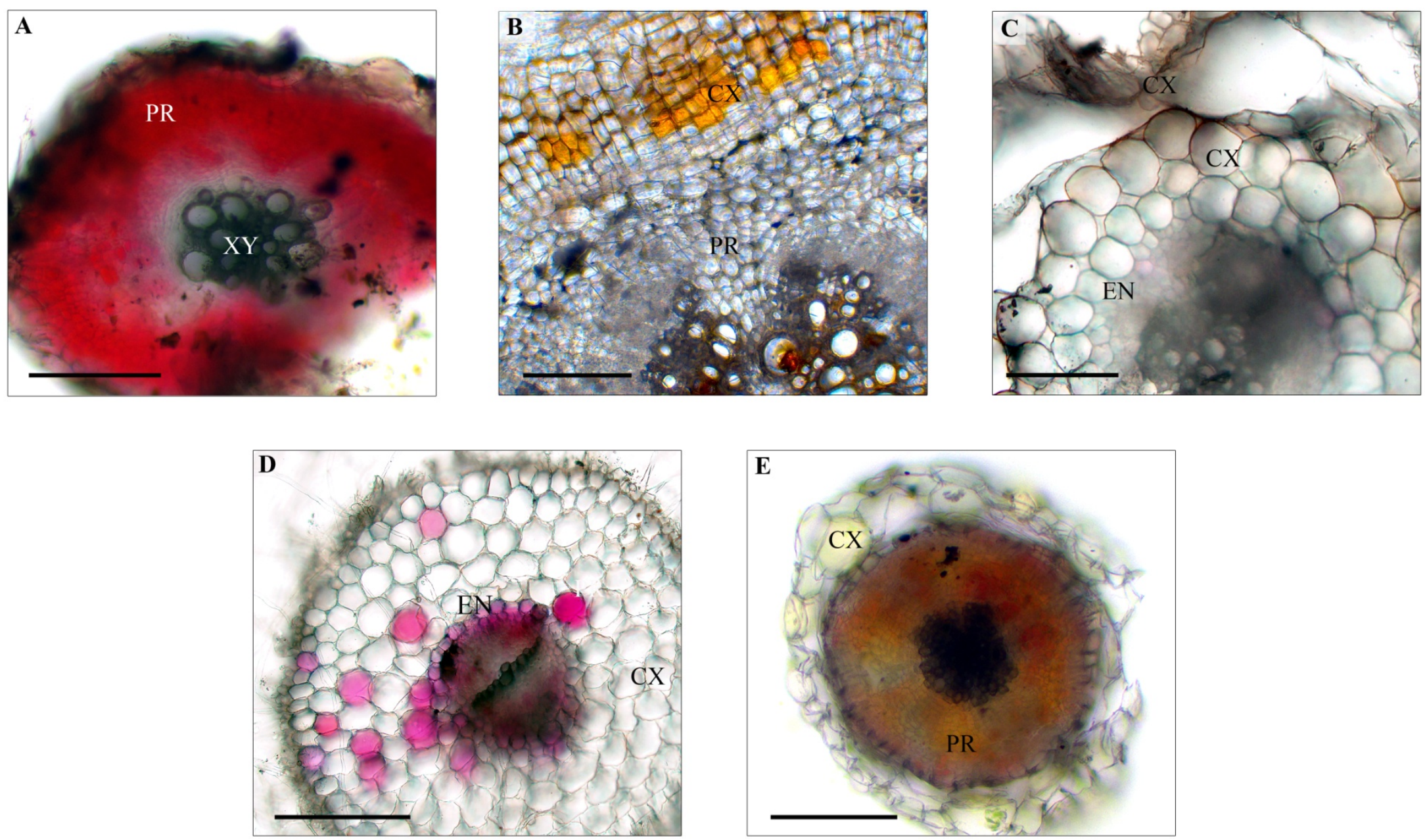

Figure 3.8 Examples of betalain-containing root sections for each species. All are transverse sections and are mounted in water. (A) Beetroot; (B) Bougainvillea; (C) Red orach; (D) Swiss chard; (E) Ulluco. Key: CX cortex, EN endodermis, PR pericycle, XY xylem. Scale bars: C = 50 $\mu \mathrm{m} ; \mathbf{A}, \mathbf{B}, \mathbf{D}$ and $\mathbf{E}=200 \mu \mathrm{m}$. All sections are unstained. 

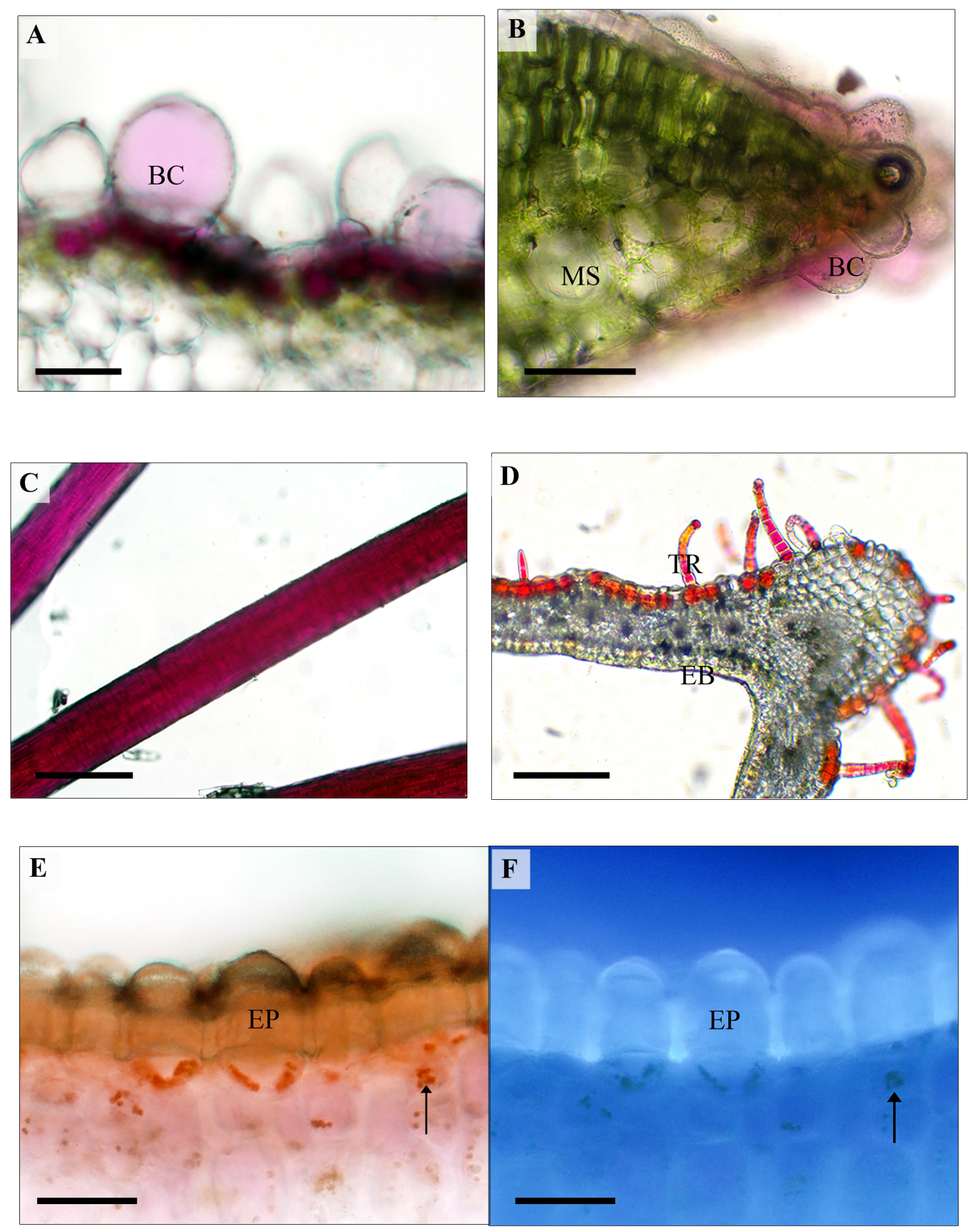

Figure 3.9 Observed pigmentation in specialised structures. All are transverse sections and are mounted in water. (A) Bladder cells on the stem of New Zealand spinach; (B) Bladder cells on the lamina margin of ulluco; (C) Pigmented spines from the stem of ruby rainbow cactus; (D) Pigmented trichomes on the fused corolla of bougainvillea; (E) White light micrograph and (F) blue light micrograph of living stone lamina, arrow indicates pigmented circular bodies; (Key: BC bladder cell, EB abaxial epidermis, ED adaxial epidermis, MP palisade mesophyll, MS spongy mesophyll, TR trichome. Scale bars: $\mathbf{A}$ and $\mathbf{B}=50 \mu \mathrm{m}, \mathbf{C}=100 \mu \mathrm{m}, \mathbf{D}$ and $\mathbf{E}=250 \mu \mathrm{m}, \mathbf{F}=500 \mu \mathrm{m}$. . All sections are unstained. 

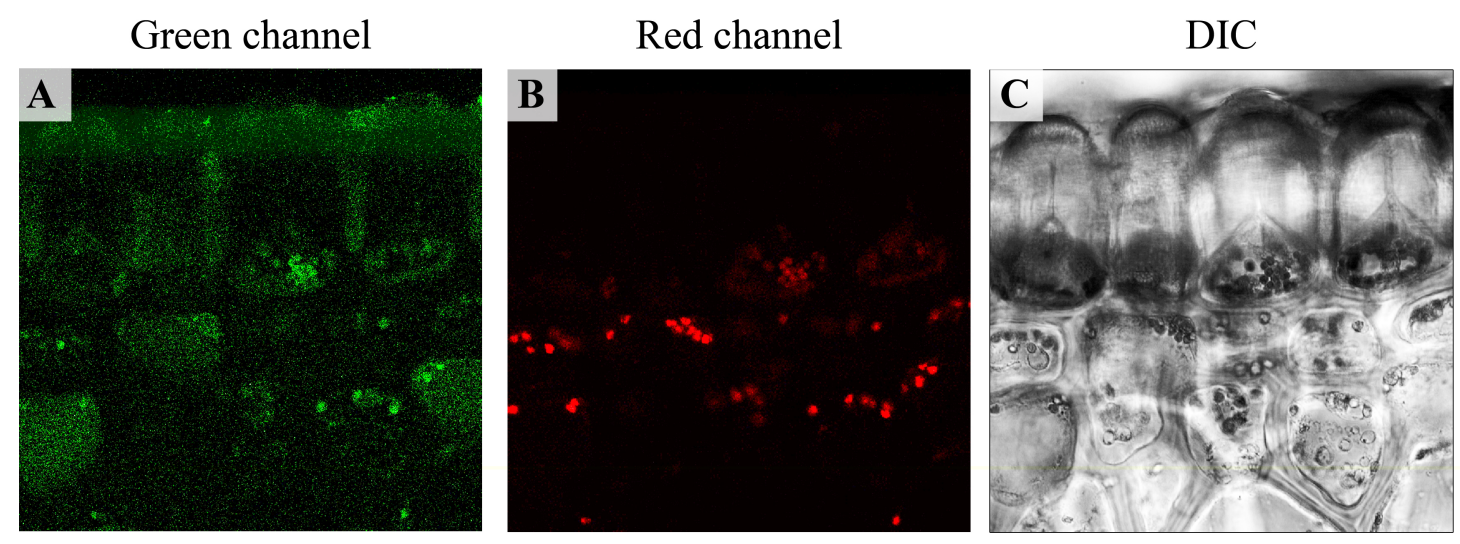

\section{Overlaid channels}

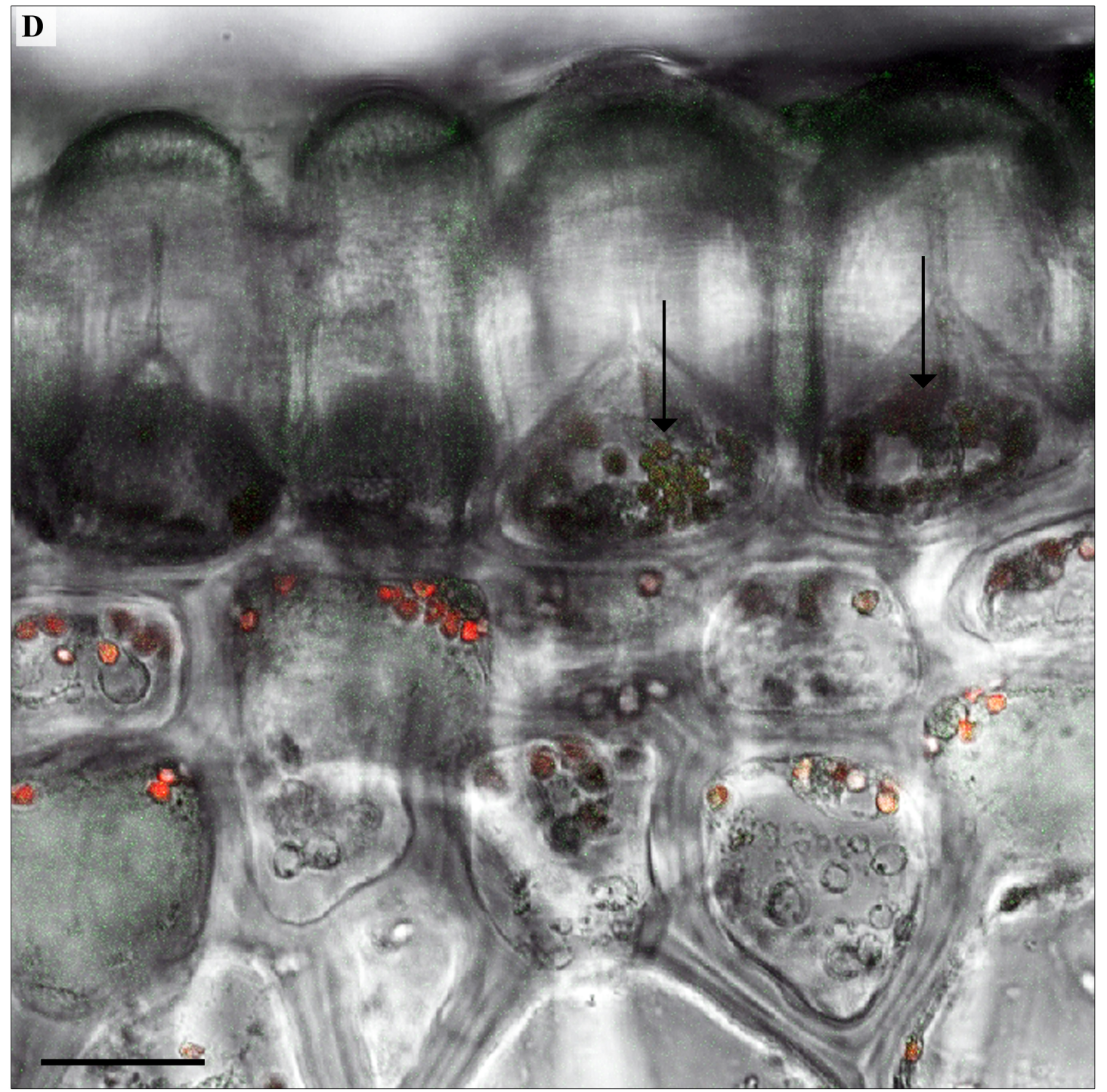

Figure 3.10 Confocal analyses of the pigmented bodies in living stone lamina cross section. Autofluorescence at $488 \mathrm{~nm}$ excitation with (A) 498 - $560 \mathrm{~nm}$ emission spectrum for betaxanthins/carotenoids (green channel) and (B) $625-732 \mathrm{~nm}$ emission spectrum for chloroplasts (red channel) overlaid with $(\mathbf{C})$ a Differential Interference Contrast (DIC) image. Arrows indicate pigmented bodies, which were likely to be plastids. Scale bar $=40 \mu \mathrm{m}$. 

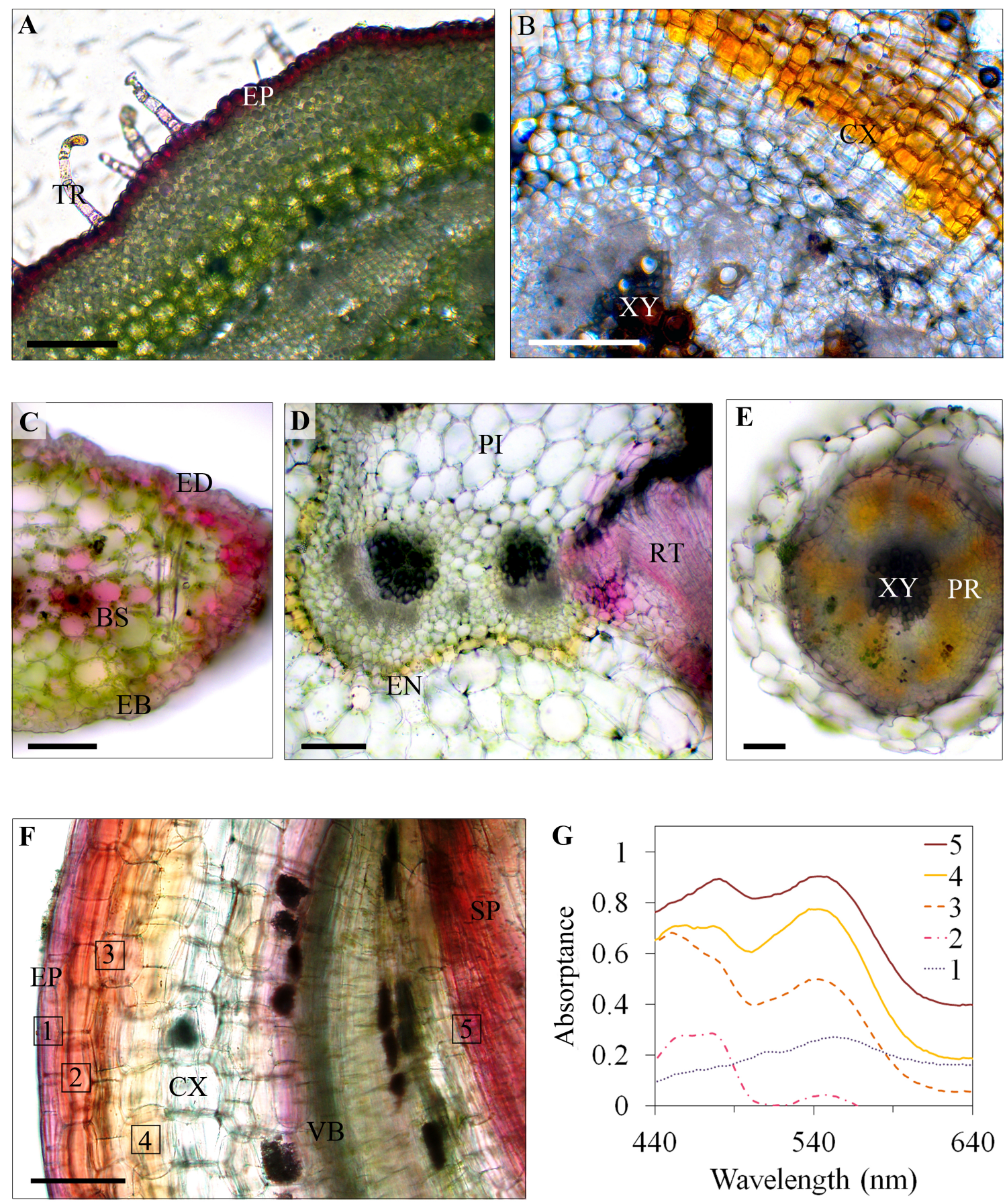

Figure 3.11 Differential localisation of betaxanthins and betacyanins across organ type and neighbouring cells. All images are of transverse sections except $\mathbf{F}$ which is from a longitudinal section. (A) Bougainvillea stem with betacyanins in epidermis and trichomes. (B) Bougainvillea root with betaxanthins in cortex. (C) Ulluco leaf containing mostly betacyanins. (D) Ulluco stem with separation of betalain compounds. (E) Ulluco root with mostly betaxanthins. (F) Red Swiss chard hypocotyl, numbered squares indicate individual vacuole absorptance readings in graph (G). Absorptance measured across the wavelength range, $440-640 \mathrm{~nm}$. Key: CX cortex, EN endodermis, EP epidermis, PI pith, RT root, VB vascular bundle. Scale bars $=150 \mu \mathrm{m}$. 
White

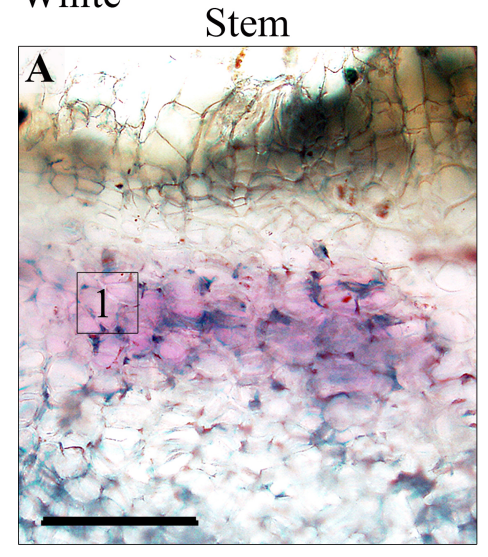

Yellow

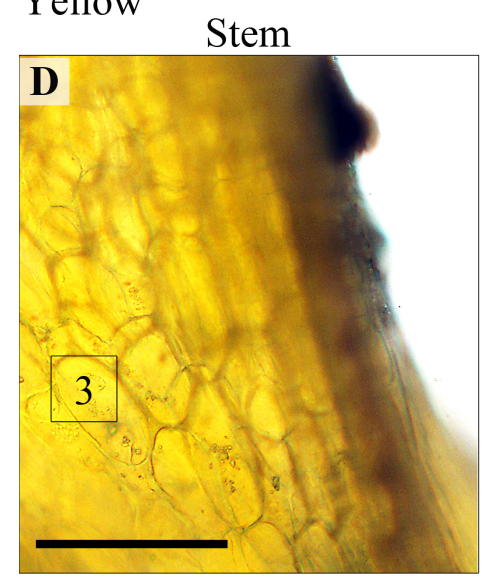

\section{Purple}

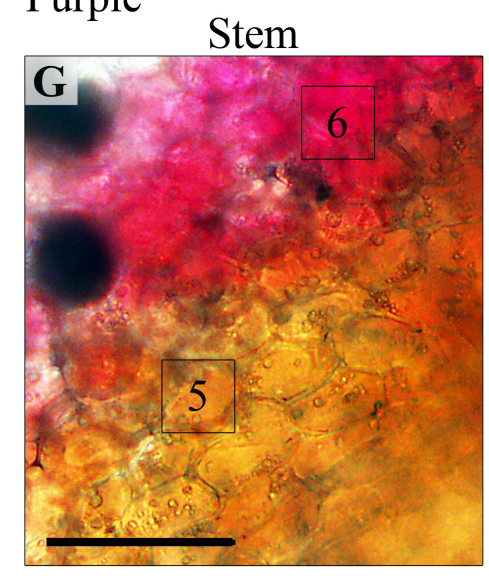

Petiole

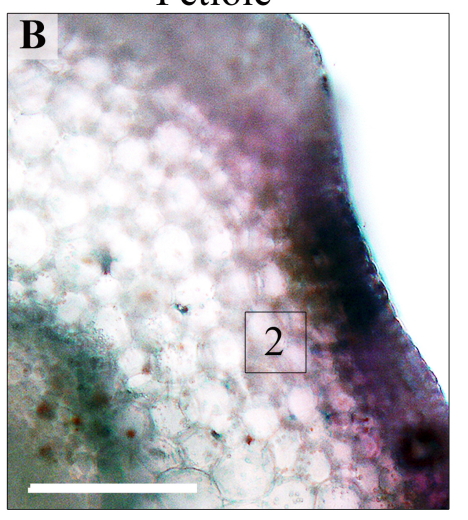

Petiole
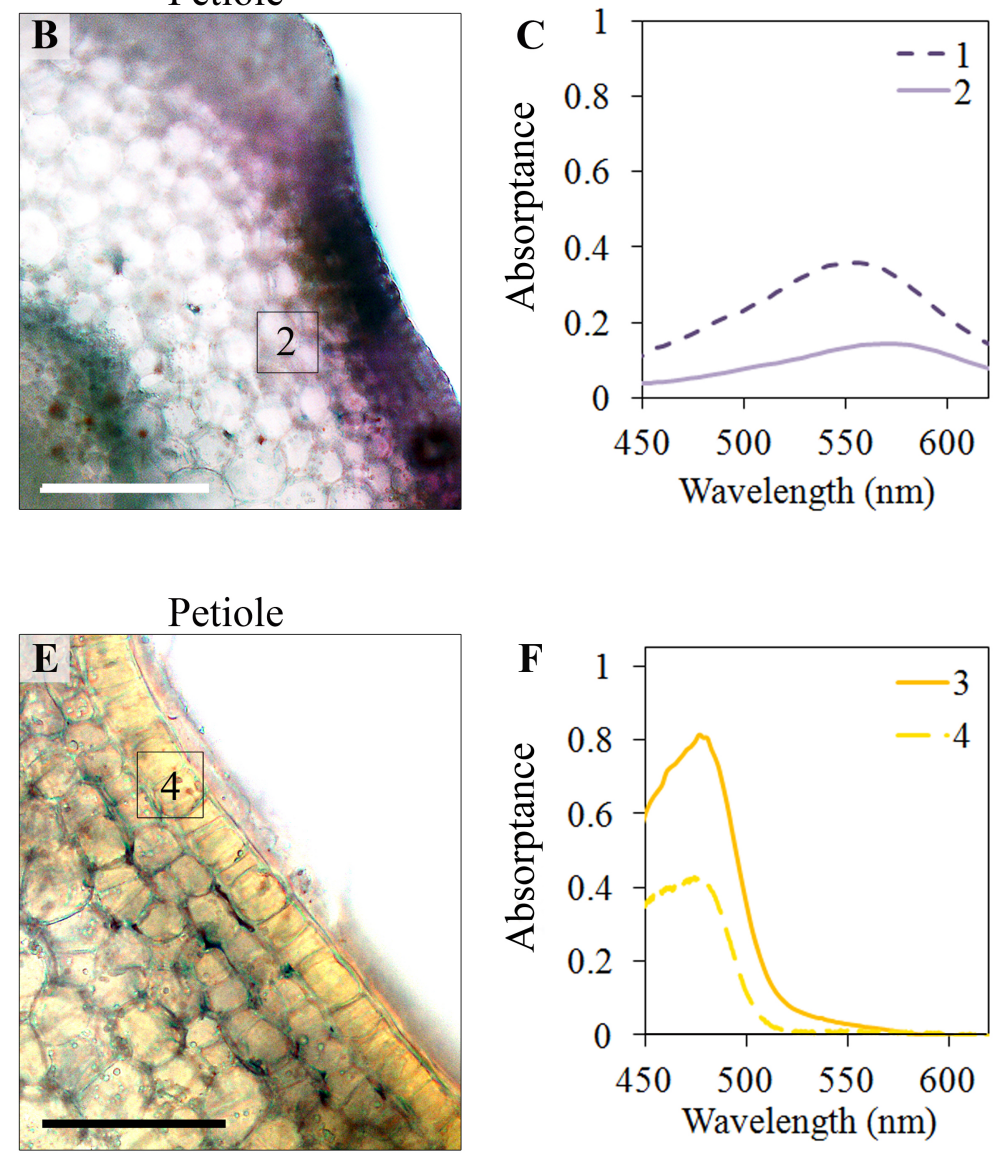

Petiole

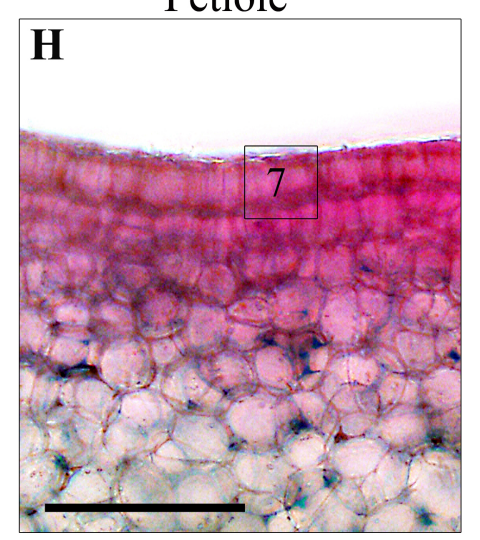

Figure 3.12 In situ absorptance measurements of individual cells in white (A, B and C), yellow (D, E and F), and purple (G, H and I) Swiss chard. Absorptance measurements were taken from transverse sections of stems (A, D and $\mathbf{G})$ and petioles of young leaves (B, E and $\mathbf{H})$ across the wavelength range, $440-640 \mathrm{~nm}(\mathbf{C}, \mathbf{F}$ and $\mathbf{I})$. Numbers in the sections refer to the relevant absorptance readings in the graphs. Scale bars: $\mathbf{A}=200$ $\mu \mathrm{m}, \mathbf{B}$ and $\mathbf{E}=50 \mu \mathrm{m}, \mathbf{D}$ and $\mathbf{G}=100 \mu \mathrm{m}, \mathbf{H}=70 \mu \mathrm{m}$. 

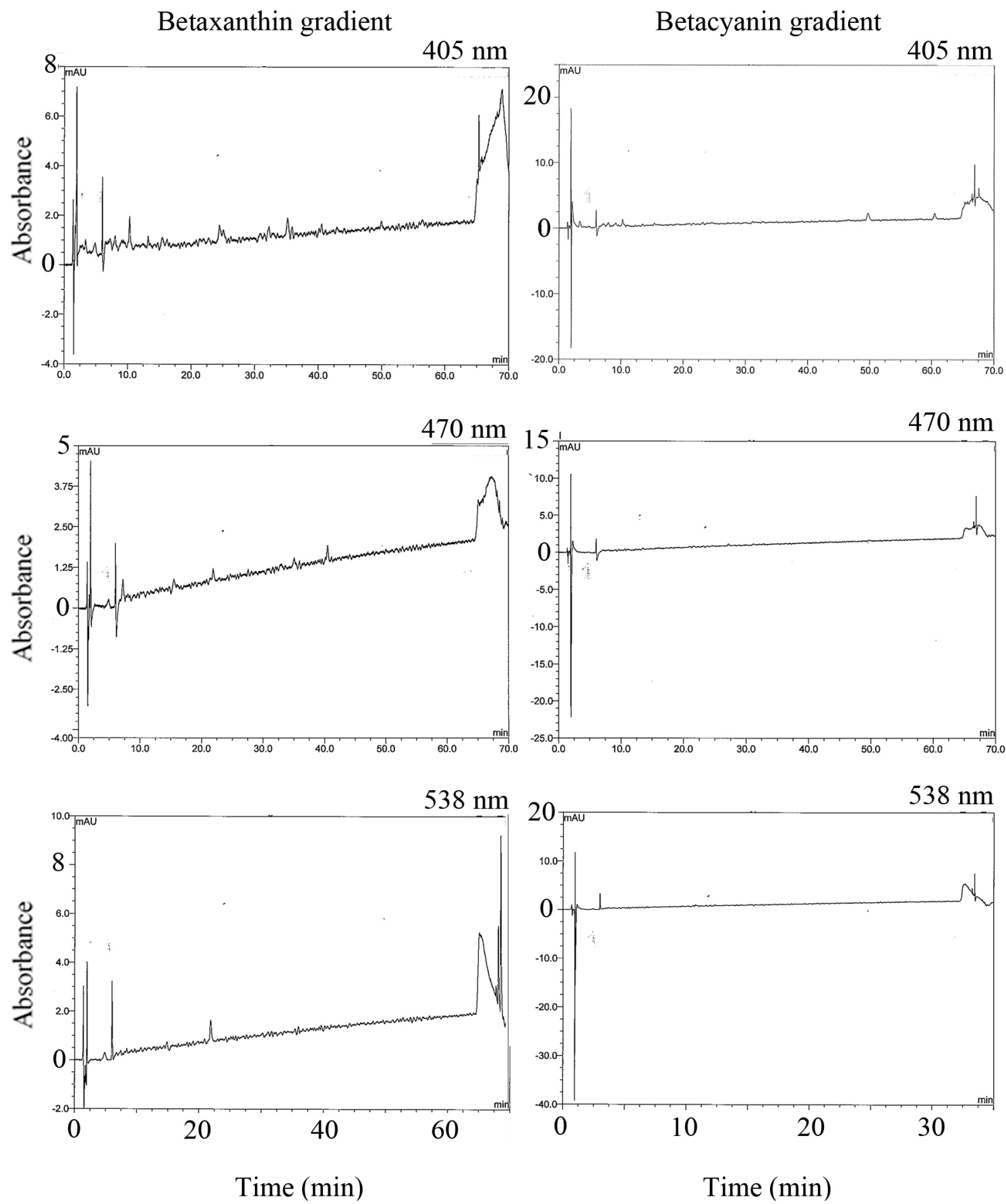

Figure 3.13 Representative chromatograms from HPLC analyses of white Swiss chard. All chromatograms are from the analysis of a young leaf extract. HPLC samples were run on a betaxanthin gradient profile and a betacyanin gradient profile. Absorbance $(\mu \mathrm{AU})$ measured with time at wavelengths $405 \mathrm{~nm}, 470 \mathrm{~nm}$ and $538 \mathrm{~nm}$. 


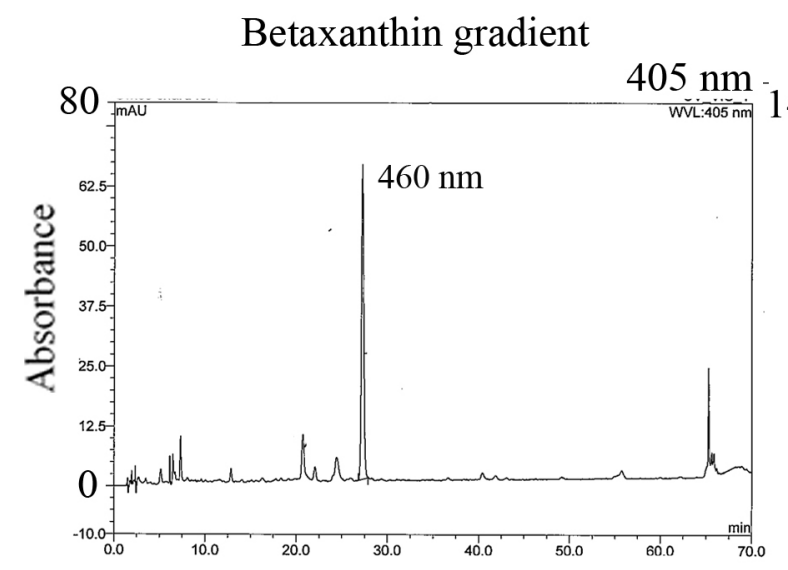

Betacyanin gradient
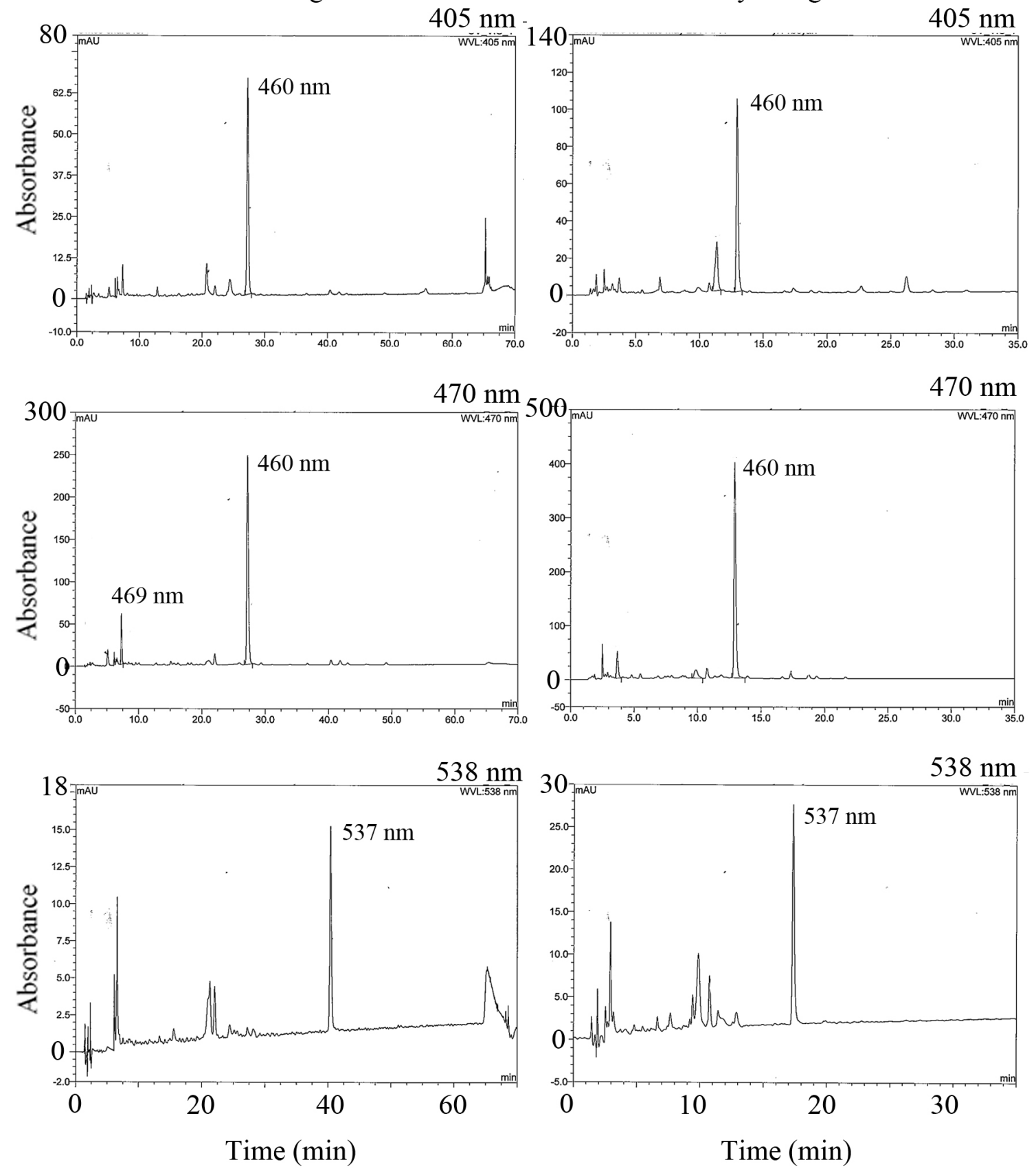

Figure 3.14 Representative chromatograms from HPLC analyses of yellow Swiss chard. All chromatograms are from the analysis of a young leaf extract. HPLC samples were run on a betaxanthin gradient profile and a betacyanin gradient profile. Absorbance $(\mu \mathrm{AU})$ measured with time at wavelengths $405 \mathrm{~nm}, 470 \mathrm{~nm}$ and $538 \mathrm{~nm}$. Individual peaks are labelled with their absorbance maximum. 

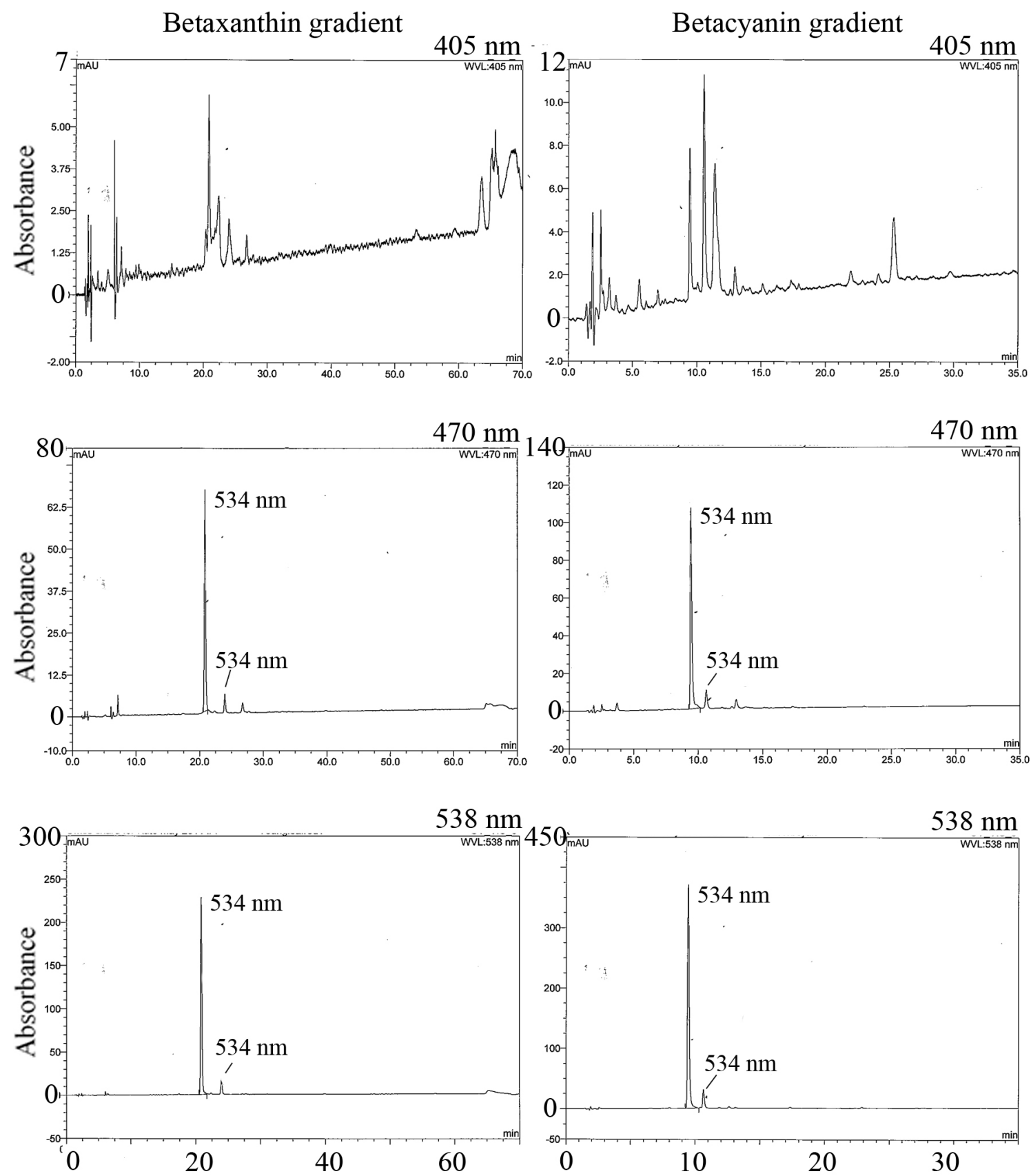

Time (min)

$538 \mathrm{~nm}$

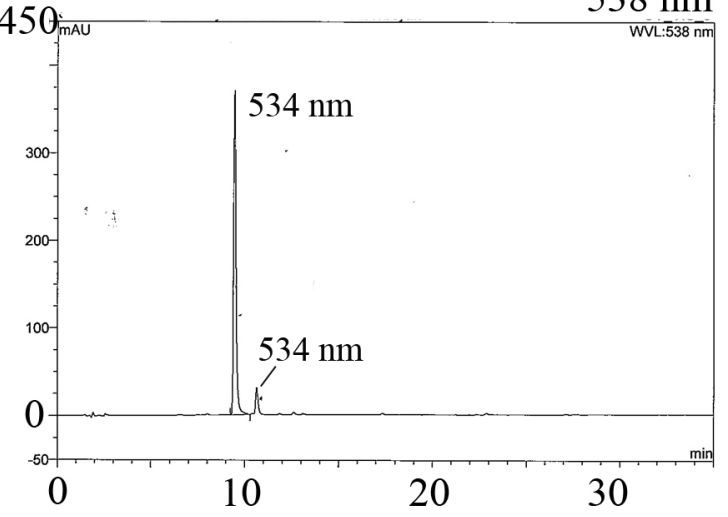

Time (min)

Figure 3.15 Representative chromatograms from HPLC analyses of purple Swiss chard. All chromatograms are from the analysis of a young leaf extract. HPLC samples were run on a betaxanthin gradient profile and a betacyanin gradient profile. Absorbance ( $\mu \mathrm{AU})$ measured with time at wavelengths $405 \mathrm{~nm}, 470 \mathrm{~nm}$ and $538 \mathrm{~nm}$. Individual peaks are labelled with their absorbance maximum. 

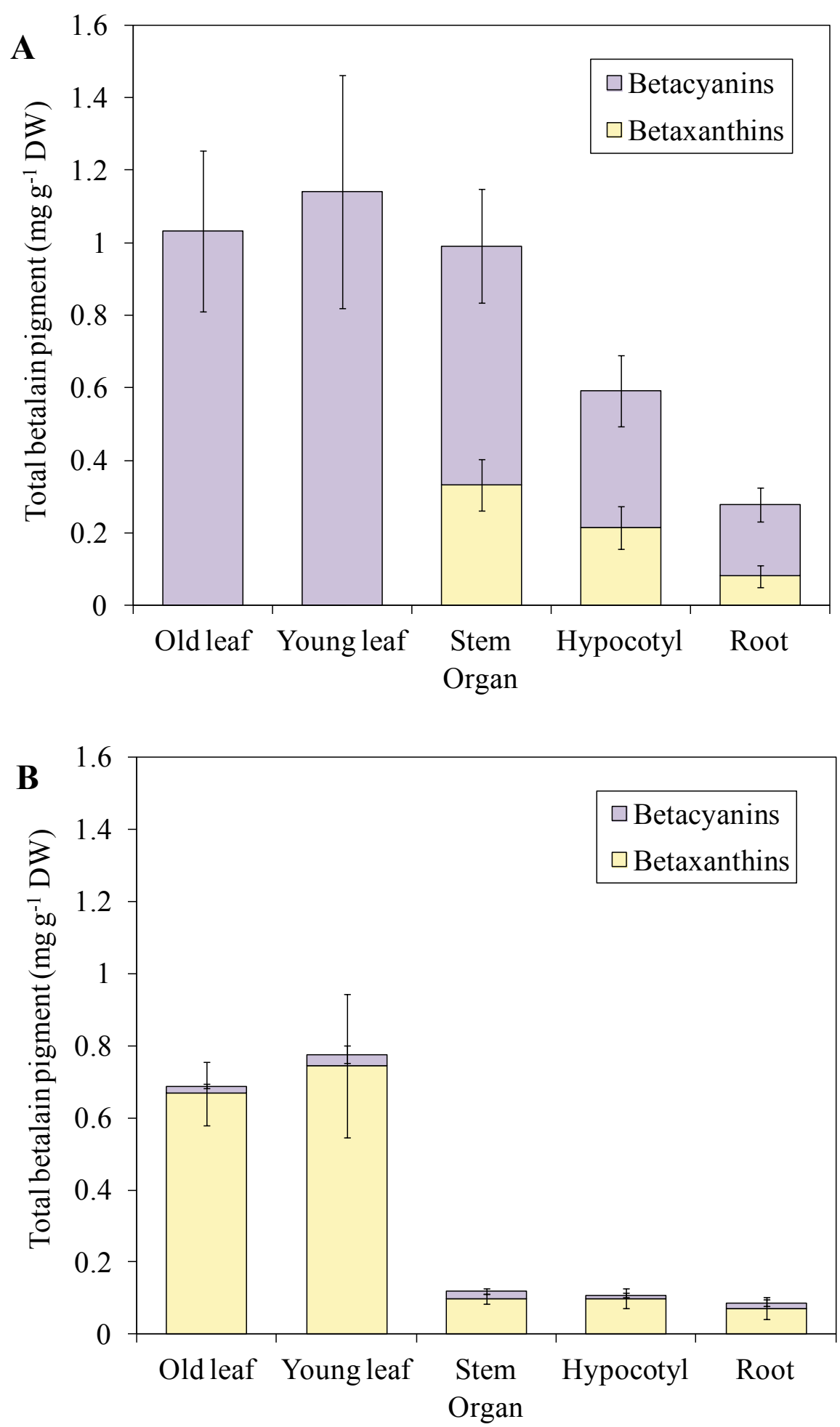

Figure 3.16 Total betalain pigment concentration $\left(\mathrm{mg} \mathrm{g}^{-1} \mathrm{DW}\right)$ in five organs from (A) purple and (B) yellow Swiss chard. Yellow and purple represent betaxanthins and betacyanins, respectively. Means \pm S.E., $n=3$. 


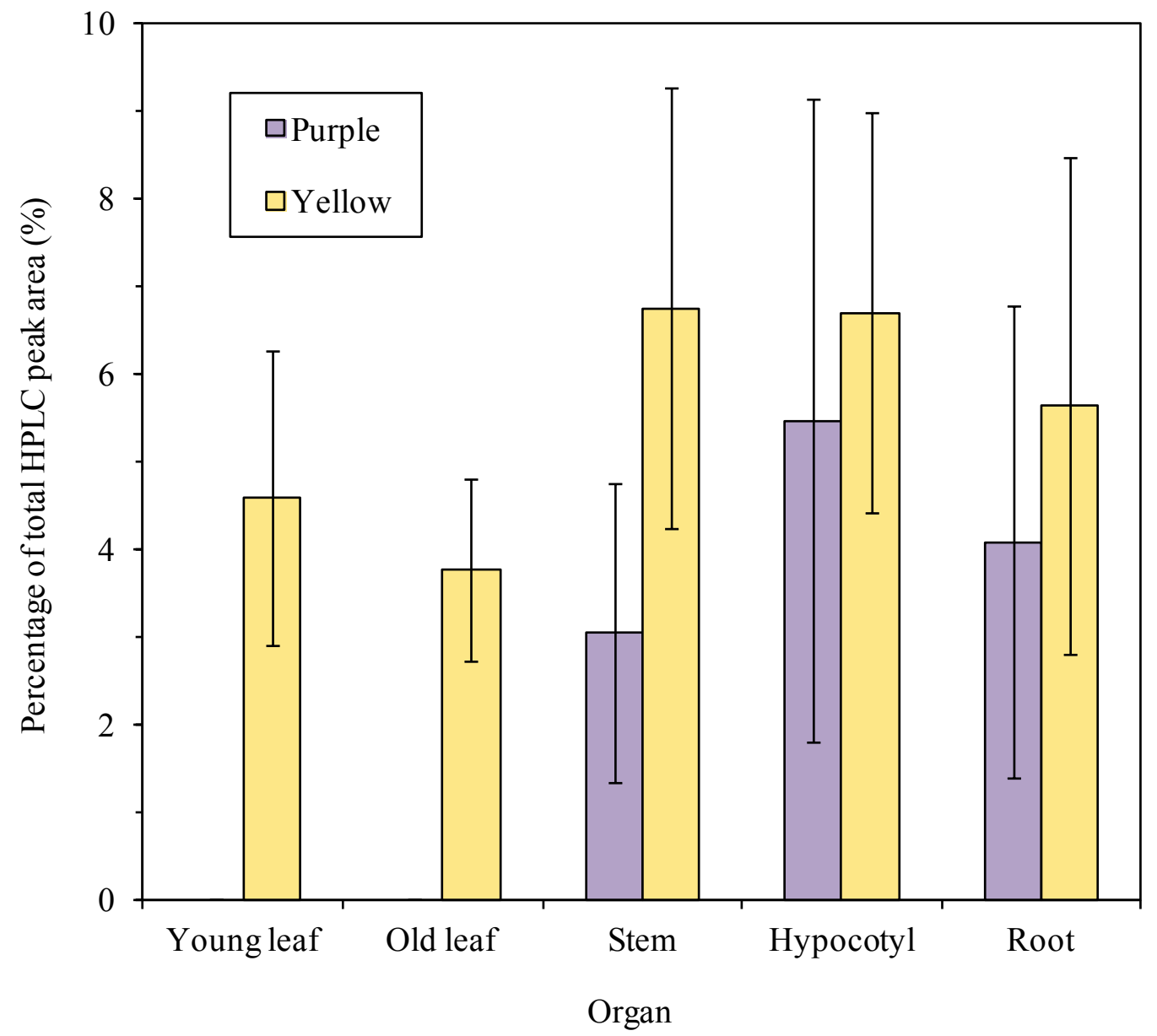

Figure 3.17 Proportion of betalamic acid in total HPLC peak area for five different organs of purple and yellow Swiss chard. Young and old leaves of the purple line had no detectable betalamic acid. Means \pm S.E., $n=3$. 


\section{Purple}

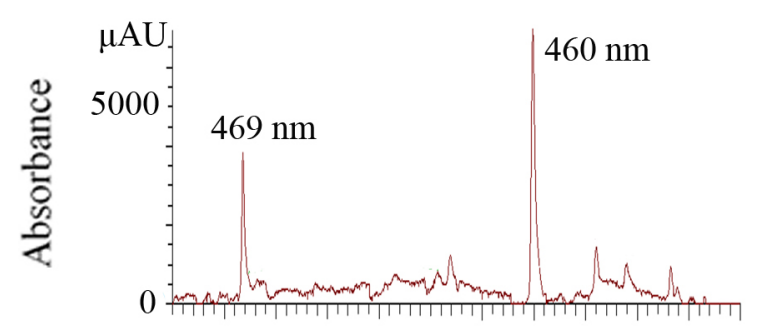

\section{Yellow}

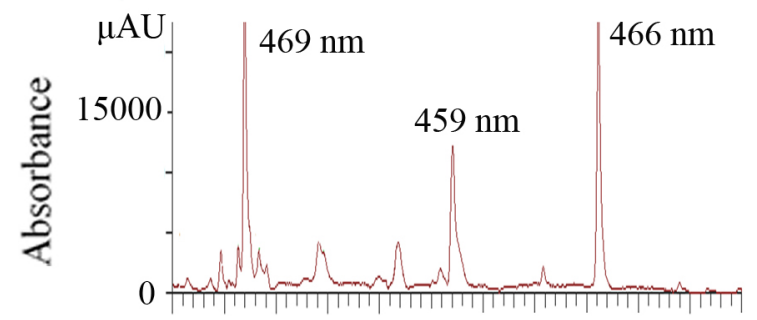

\section{Orange}

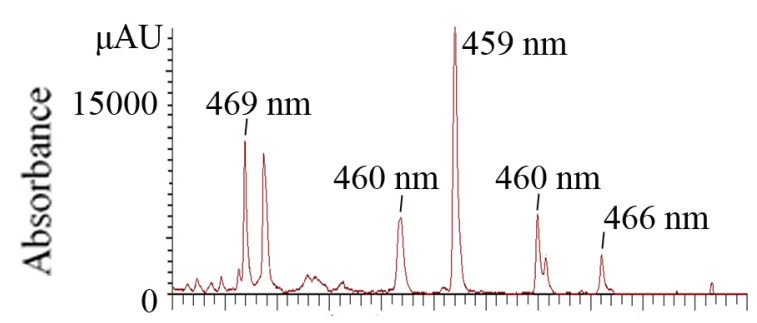

\section{Red}

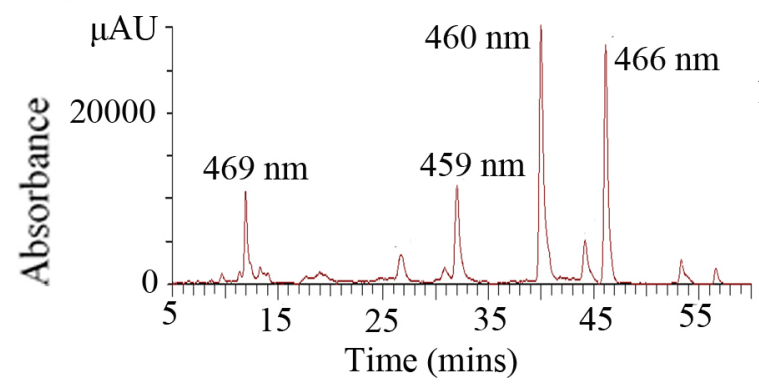

Betacyanin
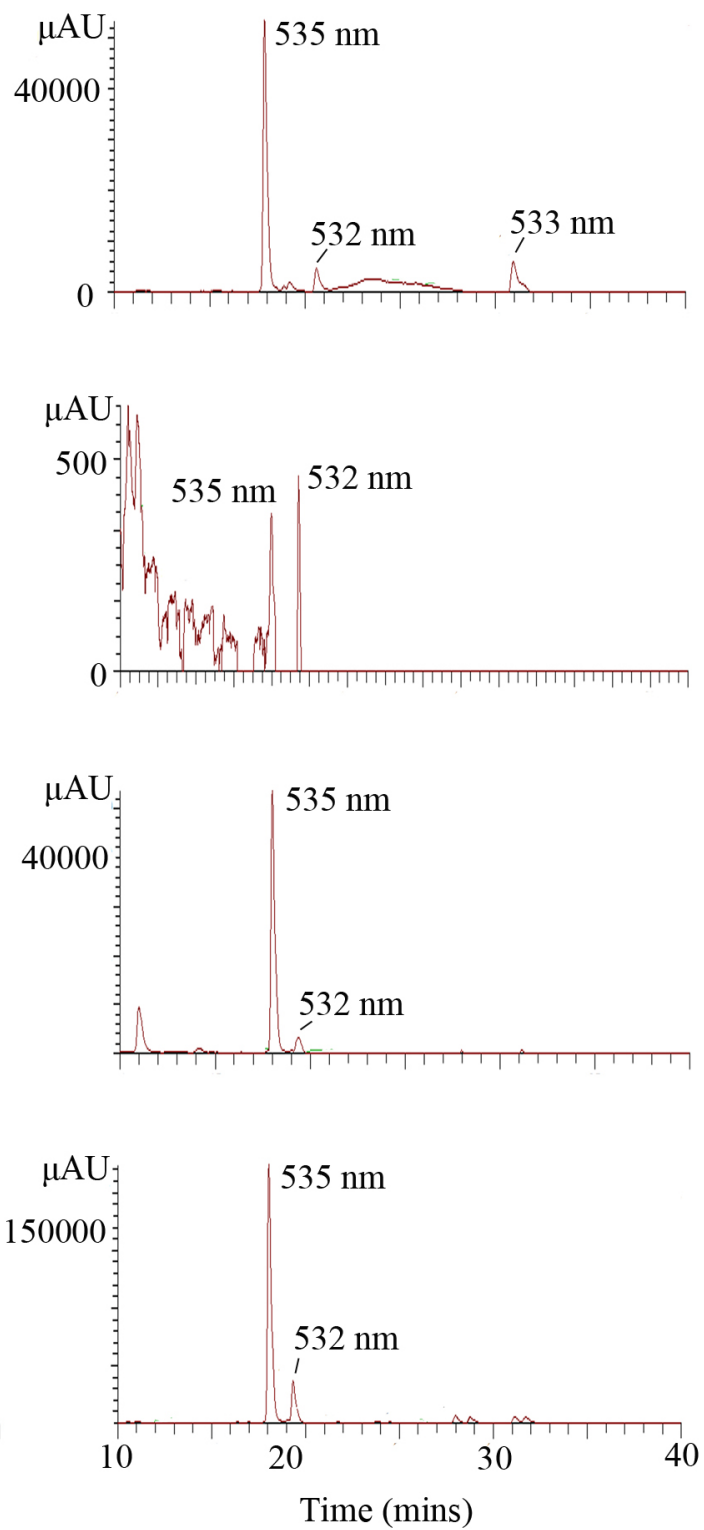

Figure 3.18 Chromatograms from LC-MS analyses for whole plant extracts of purple, yellow, orange and red Swiss chard. Absorbance ( $\mu \mathrm{AU})$ measured with time for two different gradients, betaxanthin $(470 \mathrm{~nm})$ and betacyanin $(538 \mathrm{~nm})$. Individual peaks are labelled with their absorbance maximum. 
Table 3.2 Betalain compounds identified using LC-MS in purple, yellow, orange and red Swiss chard plants

\begin{tabular}{|c|c|c|c|c|c|c|c|}
\hline ID (alternate name) & Purple & Yellow & Orange & Red & $\begin{array}{c}\mathrm{RT} \\
(\mathrm{min})\end{array}$ & $\begin{array}{l}\lambda_{\operatorname{Max}} \\
(\mathrm{nm})\end{array}$ & $\begin{array}{c}\mathrm{m} / \mathrm{z}, \\
{[\mathrm{M}+\mathrm{H}]^{+}}\end{array}$ \\
\hline \multicolumn{8}{|l|}{ Betaxanthin gradient } \\
\hline 1. Serine-bx & $\operatorname{tr}$ & + & $\operatorname{tr}$ & $\operatorname{tr}$ & 9.72 & 470 & 299 \\
\hline $\begin{array}{l}\text { 2. Glutamine-bx } \\
\text { (vulgaxanthin I) }\end{array}$ & + & +++ & ++ & ++ & 11.84 & 469 & 340 \\
\hline 3. Glycine-bx & - & $\operatorname{tr}$ & - & $\operatorname{tr}$ & 13.18 & 468 & 269 \\
\hline $\begin{array}{l}\text { 4. Glutamate-bx } \\
\text { (vulgaxanthin II) }\end{array}$ & - & + & - & $\operatorname{tr}$ & 19.26 & 470 & 341 \\
\hline 5. $\gamma$-Aminobutyric acid-bx & - & + & $\operatorname{tr}$ & + & 31.01 & 460 & 297 \\
\hline 6. Proline-bx & - & + & - & - & 33.18 & 467 & 309 \\
\hline 7. Dopamine-bx & $\operatorname{tr}$ & +++ & + & +++ & 46.2 & 459 & 347 \\
\hline 8. 3-Methoxytyramine-bx & $\operatorname{tr}$ & $\operatorname{tr}$ & + & + & 56.73 & 462 & 361 \\
\hline 9. 17-Decarboxy-betanidin & - & + & ++ & + & 26.94 & 466 & 345 \\
\hline 10. 15-Decarboxy-betanidin & - & ++ & +++ & ++ & 32.07 & 466 & 345 \\
\hline \multicolumn{8}{|l|}{ Betacyanin gradient } \\
\hline $\begin{array}{l}\text { 11. Betanidin-5- } O \text {-glucoside } \\
\text { (betanin) }\end{array}$ & +++ & $\operatorname{tr}$ & +++ & +++ & 17.97 & 535 & 551 \\
\hline 12. Isobetanin & $\operatorname{tr}$ & - & + & + & 19.43 & $\begin{array}{l}532 \\
331\end{array}$ & 551 \\
\hline 13. Betanidin & - & - & - & - & & 532 & 389 \\
\hline 14. Isobetanidin & - & - & - & - & & 540 & 389 \\
\hline 15. Phyllocactin & + & - & - & - & 20.60 & 532 & 637 \\
\hline $\begin{array}{l}\text { 16. Betanidin-monoferuloyl- } \\
\text { 5-O- } \beta \text {-diglucoside }\end{array}$ & - & - & $\operatorname{tr}$ & + & 28.07 & $\begin{array}{c}327 \\
532\end{array}$ & 889 \\
\hline $\begin{array}{l}\text { 17. Isobetanidin- } \\
\text { monoferuloyl-5-O- } \beta \text { - } \\
\text { diglucoside }\end{array}$ & - & - & - & + & 28.84 & $\begin{array}{c}327 \\
532\end{array}$ & 889 \\
\hline 18. Lampranthin II & ++ & - & + & + & 31.2 & $\begin{array}{l}533 \\
330\end{array}$ & 727 \\
\hline 19. Isolampranthin II & $\operatorname{tr}$ & - & + & + & 31.7 & $\begin{array}{l}532 \\
326\end{array}$ & 727 \\
\hline
\end{tabular}

Key: Numbering of compounds is associated with structures in Figures 3.19-3.21; $+++=$ Most abundant $(>15000 \mu \mathrm{AU}) ;++=$ abundant $(>5000 \mu \mathrm{AU}) ;+=$ present but minor; $\operatorname{tr}=$ just detectable; $-=$ not detected. 
<smiles>O=C(O)C1=C/C(=C/C=[NH+]/C(CO)C(=O)[O-])C[C@@H](C(=O)O)N1</smiles>

Serine-betaxanthin<smiles>NC(=O)CCC([NH+]=CC=C1C=C(C(=O)O)N[C@H](C(=O)O)C1)C(=O)[O-]</smiles>

Glutamine-betaxanthin<smiles>O=C([O-])C[NH+]=CC=C1C=C(C(=O)O)N[C@H](C(=O)O)C1</smiles>

Glycine-betaxanthin<smiles>O=C(O)CC[C@@H](/[NH+]=C\C=C1/C=C(C(=O)O)N[C@H](C(=O)O)C1)C(=O)[O-]</smiles>

Glutamate-betaxanthin<smiles>O=C([O-])CCC[NH+]=CC=C1C=C(C(=O)O)N[C@H](C(=O)O)C1</smiles>

$\gamma$-Aminobutyric acid-betaxanthin
6.<smiles>O=C(O)C1=C/C(=C/C=[N+]2CCCC2C(=O)[O-])C[C@H](C(=O)O)N1</smiles>

Proline-betaxanthin
7.<smiles>O=C(O)C1=C/C(=C/C=[NH+]\CCc2ccc(O)c(O)c2)C[C@H](C(=O)O)N1</smiles>

Dopamine-betaxanthin<smiles>COc1cc(CC/[NH+]=C/C=C2/C=C(C(=O)O)N[C@H](C(=O)O)C2)ccc1O</smiles>

3-Methoxytyramine-bx

Figure 3.19 Betaxanthin compounds identified using LC-MS and HPLC analyses. Blue indicates amine or amino group on the betalamic acid moiety. Adapted from Strack et al. (2003), Bohm and Mack (2004), and Stintzing and Carle (2007). 
9.<smiles>O=C([O-])[C@@H]1Cc2cc(O)c(O)cc2[N+]1=CC=C1C=CN[C@@H](C(=O)O)C1</smiles>

17-Descarboxy betanidin
10.<smiles>O=C(O)C1=CC(=CC=[N+]2c3cc(O)c(O)cc3C[C@H]2C(=O)[O-])CCN1</smiles>

15-Descarboxy betanidin<smiles>C=C1C=C(C(=O)O)NC(C(=O)OCCCCC(=O)O)C1</smiles>

13.

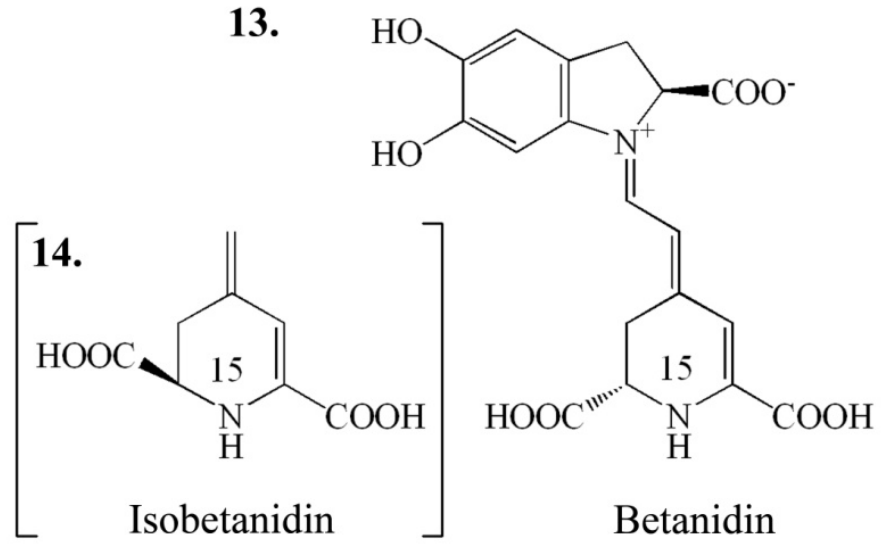

Figure 3.20 Betacyanin compounds identified using LC-MS and HPLC analyses. Blue indicates the addition of a sugar glucoside of the betanidin moiety. Adapted from Strack et al. (2003) and Herbach et al. (2006). 


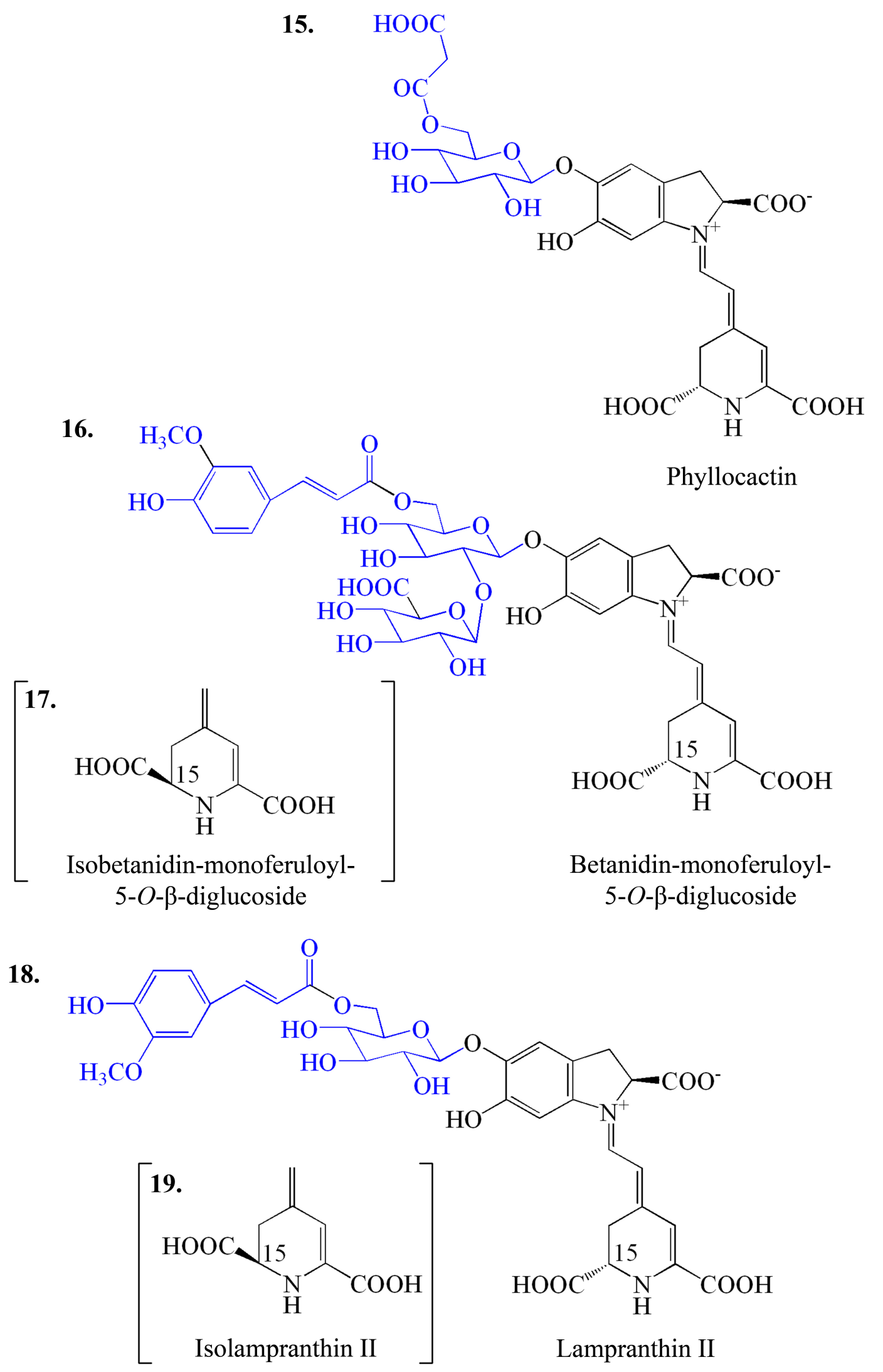

Figure 3.21 Betacyanin compounds identified using LC-MS and HPLC analyses (continued). Blue indicates the addition of sugar glucosides and hydroxycinnamic acids to the betanidin moiety. Adapted from Herbach et al. (2005), Schliemann and Strack (1998) and Strack et al. (1988). 
Table 3.3 Absolute quantity of compounds identified with HPLC from five organs of purple Swiss chard.

\begin{tabular}{|c|c|c|c|c|c|c|c|c|c|c|c|c|c|c|c|c|}
\hline \multirow[b]{3}{*}{ Organ } & \multicolumn{16}{|c|}{ Betalainic compound - mean absolute values in $\mathrm{mg} \mathrm{g}^{-1} \mathrm{DW} \pm$ S.E. ${ }^{a, b}$} \\
\hline & \multicolumn{7}{|c|}{ Betaxanthins } & \multicolumn{9}{|c|}{ Betacyanins } \\
\hline & 2 & 3 & 4 & 5 & 6 & 7 & 8 & 9 & 10 & 11 & 12 & 13 & 14 & 15 & 18 & 19 \\
\hline Old Leaf & - & - & - & - & - & - & - & - & - & $0.7 \pm 0.3$ & $0.2 \pm 0.1$ & - & - & - & $0.2 \pm 0.2$ & $0.1 \pm 0.1$ \\
\hline Young leaf & - & - & - & - & - & - & - & - & - & $1.1 \pm 0.3$ & $0.1 \pm 0.1$ & - & - & - & $0.1 \pm 0.1$ & - \\
\hline Stem & $0.2 \pm 0.1$ & - & - & - & - & $0.3 \pm 0.1$ & - & - & - & $0.2 \pm 0.1$ & $0.1 \pm 0.1$ & $0.2 \pm 0.2$ & $\operatorname{tr}$ & $\operatorname{tr}$ & - & - \\
\hline Hypocotyl & $0.2 \pm 0.1$ & - & - & - & - & $\operatorname{tr}$ & - & - & - & $0.1 \pm 0.1$ & $\operatorname{tr}$ & $0.1 \pm 0.1$ & - & - & - & - \\
\hline Root & $0.1 \pm 0.1$ & - & - & - & - & $\operatorname{tr}$ & - & - & - & $0.1 \pm 0.1$ & $\operatorname{tr}$ & $0.1 \pm 0.1$ & - & - & - & - \\
\hline
\end{tabular}

Key: Compounds numbers designated in Table 3.2. ${ }^{a}$ (tr) Trace values under $0.025 \mathrm{mg} \mathrm{g}^{-1}$ DW. ${ }^{b}(-)$ Absolute values under $0.005 \mathrm{mg} \mathrm{g}^{-1} \mathrm{DW}$ or not detected. Betalain compound key: 2. Glutamine-bx; 3. Glycine-bx; 4. Glutamate-bx; 5. $\gamma$-Aminobutyric acid-bx; 6. Proline-bx; 7. Dopamine-bx; 8. 3-Methoxytyramine-bx; 9. 17-Decarboxy-betanidin; 10. 15-Decarboxy-betanidin; 11. Betanin; 12. Isobetanin; 13. Betanidin; 14. Isobetanidin; 15. Phyllocactin; 18. Lampranthin II; 19. Isolampranthin II 
Table 3.4 Absolute quantity of compounds identified with HPLC from five organs of yellow Swiss chard.

Betalainic compound - mean absolute values in $\mathrm{mg} \mathrm{g}^{-1} \mathrm{DW} \pm$ S.E. ${ }^{a, b}$

\begin{tabular}{|c|c|c|c|c|c|c|c|c|c|c|c|c|c|c|c|c|}
\hline Organ & 2 & \multicolumn{6}{|c|}{ Betaxanthins } & \multicolumn{9}{|c|}{ Betacyanins } \\
\hline Old Leaf & $0.2 \pm 0.1$ & $0.1 \pm 0.1$ & $0.1 \pm 0.1$ & - & $0.1 \pm 0.1$ & $0.4 \pm 0.2$ & $\operatorname{tr}$ & $\operatorname{tr}$ & $\operatorname{tr}$ & - & - & - & - & - & - & - \\
\hline Young leaf & $0.3 \pm 0.2$ & $0.1 \pm 0.1$ & $\operatorname{tr}$ & $\operatorname{tr}$ & $0.1 \pm 0.1$ & $0.3 \pm 0.1$ & - & $\operatorname{tr}$ & $\operatorname{tr}$ & - & - & - & - & - & - & - \\
\hline Hypocotyl & $0.1 \pm 0.1$ & - & - & - & - & $0.1 \pm 0.1$ & - & - & - & $\operatorname{tr}$ & - & - & - & - & - & - \\
\hline Root & $0.1 \pm 0.1$ & - & - & - & - & $\operatorname{tr}$ & - & - & - & $\operatorname{tr}$ & - & - & - & - & - & - \\
\hline
\end{tabular}

Key: Compounds numbers designated in Table 3.2. ${ }^{a}$ (tr) Trace values under $0.025 \mathrm{mg} \mathrm{g}^{-1} \mathrm{DW} .{ }^{b}(-)$ Absolute values under $0.005 \mathrm{mg} \mathrm{g}{ }^{-1} \mathrm{DW}$ or not detected. Betalain compound key: 2. Glutamine-bx; 3. Glycine-bx; 4. Glutamate-bx; 5. $\gamma$-Aminobutyric acid-bx; 6. Proline-bx; 7. Dopamine-bx; 8. 3-Methoxytyramine-bx; 9. 17-Decarboxy-betanidin; 10. 15-Decarboxy-betanidin; 11. Betanin; 12. Isobetanin; 13. Betanidin; 14. Isobetanidin; 15. Phyllocactin; 18. Lampranthin II; 19. Isolampranthin II 


\subsection{Discussion}

This study presents a more comprehensive analysis of betalain pigment localisation within different plant organs than any previously published. The 12 species/subspecies analysed were representatives from five different families (Amaranthaceae, Aiozaceae, Basellaceae, Cactaceae and Nyctaginaceae) and covered a wide range of morphology. The pigmentation patterns appeared similar in many species, despite morphological differences and the inclusion of a couple of cultivars bred for enhanced pigmentation (Swiss chard and beetroot). Betalain pigment occurred within dermal, ground, and vascular tissues.

\subsubsection{Developmental origin of similar pigmented tissues}

Betalain pigments were found in similar tissue types within multiple organs on a single plant. The occurrence of similar pigmentation patterns raises the question of whether these tissues produce pigment during their development. From the results in this study it is difficult to correlate pigmentation patterns in different tissues with their developmental origin. Although four species were sectioned as seedlings and mature plants, the sectioning of most species was made from either seedlings or mature plants. The exact timing of when betalain pigment production occurs within these tissues is, therefore, largely unknown. Pigmentation could have been externally induced after tissue development was complete. To draw conclusions on developmental timing, observations of more developmental stages are required.

\subsubsection{Betalain localisation is similar to anthocyanins}

The pigmentation patterns in vegetative tissues of betalainic plants are similar to those of anthocyanins in plants. Many anthocyanic species are reported to have multiple pigmented organs on the same plant (Blank, 1947; Lee \& Collins, 2001; Thakur \& Nozzolillo, 1978). Within these pigmented organs, anthocyanins are localised to specific dermal, ground and vascular tissues.

Similar to betalains, anthocyanin pigmentation in dermal and ground tissues are the most common out of the three tissue types. Epidermal pigmentation is widely reported in the organs of many anthocyanin-producing species, particularly of leaves (Gould \& Quinn, 1999; Kytridis \& Manetas, 2006; Lee \& Collins, 2001; Li et al., 2008; 
Nozzolillo, 1973; Nozzolillo \& McNeill, 1985; Thakur \& Nozzolillo, 1978).

Anthocyanins have also been found in the epidermis of stems (Gould et al., 2010; Yamazaki et al., 2003), hypocotyls (Nozzolillo \& McNeill, 1985; Troyer, 1964), and petioles (Gould \& Quinn, 1999). Common pigmented ground tissues include foliar palisade and mesophyll tissues (Gould et al., 2002; Kytridis \& Manetas, 2006; Lee \& Collins, 2001), stem pith cells (Li et al., 2008), as well as cortex tissues in hypocotyls (Nozzolillo, 1973; Nozzolillo \& McNeill, 1985), stems (Gould et al., 2010) and roots (Solangaarachchi \& Gould, 2001). Vascular tissue pigmentation has also been observed in anthocyanic plants. However, this pigmentation usually neighbours vascular tissues such as in foliar bundle sheath cells (Kytridis \& Manetas, 2006; Lee \& Collins, 2001), in epidermal cells directly above veins on leaves (Goldsbrough et al., 1994), sepals, and petals (Albert et al., 2011; Bradley et al., 1998; Shang et al., 2011; Yamagishi et al., 2010), rather than within vascular bundles. The few recorded occurrences of anthocyanic pigmentation within vascular tissues include vascular parenchyma of leaves (Gould \& Quinn, 1999; Lee \& Collins, 2001), phloem parenchyma in the stem of Monotropa hypopitys var. sanguinea Hausskn (Blank, 1947), in the roots of Metrosideros excelsa (Solangaarachchi \& Gould, 2001), as well as in the branches and petioles in a couple species of Prunus (Li et al., 2008).

Similarities in pigment distribution of betalains and anthocyanins may indicate shared inducible biosynthetic elements, even though they have different biosynthetic pathways. In addition to similarities in pigment distribution, biosynthesis of betalains and anthocyanins has been observed at similar developmental stages as well as in response to environmental factors. Transient reddening of developing vegetative tissues occurs in both young anthocyanic (Dominy et al., 2002; Steyn et al., 2002) and betalainic (Adams et al., 1998) plants. Further evidence for similar inducible biosynthetic elements is that both betalain and anthocyanin production is induced by similar environmental factors (Stafford, 1994). Biosynthesis of betalain and anthocyanin pigments is induced by factors such as light quality (Ibdah et al., 2002; Kishima et al., 1995; Kubasek et al., 1992) and high light levels (Hughes et al. (2005); Vogt et al. (1999); observations of New Zealand spinach and bougainvillea, Figure 1.4). 


\subsubsection{Betalainic compound distribution is uneven within and between organs}

Analyses of pigment localisation in bougainvillea, Swiss chard and ulluco revealed uneven distribution of betaxanthins and betacyanins between and within organs on the same plant. Biosynthesis of these two pigment types are strongly linked, as they both contain the chromophore betalamic acid (Girod \& Zrÿd, 1991). In spite of this linkage, it appears that betacyanins may contribute the most to overall betalain content. Betalainic plants with higher levels of betacyanin often contain higher overall pigment levels (Opuntia spp., Stintzing et al. (2005); beet, Kugler et al. (2004) and Girod and Zrÿd (1991); Gomphrena spp., Kugler et al. (2007)).

It was hypothesised in the beet cell culture study by Girod and Zrÿd (1991), that the biosynthesis of betaxanthins and betacyanins is limited by precursor level. Differently coloured cell lines produced different levels of total betalain: where they were higher in red and violet phenotypes than in yellow and orange lines. As the conjugation reaction of both betaxanthins and betacyanins is considered spontaneous (Schliemann et al., 1999), the red and violet lines should have had to have greater initial levels of both cyclo-DOPA and betalamic acid than the yellow and orange lines. From these results, Girod and Zrÿd (1991) suggested that synthesis of cyclo-DOPA and betalamic acid are co-regulated for the enhanced production of betacyanins. In support of this, Kugler et al. (2004) found that purple, red, yellow and yellow-orange Swiss chard leaves all contained similar levels of betaxanthins, but differed in betacyanin concentration. Similar to those results, in my study purple Swiss chard organs that contained mostly betacyanins were higher in total betalain content than similar organs on the yellow plants. Based on the hypothesis of betalamic acid/cyclo-DOPA up-regulation, betacyanic tissues should have higher levels of betalamic acid than betaxanthic tissues. In yellow and purple Swiss chard organs, the opposite was found. Detectable levels of betalamic acid were only found in organs where betaxanthins were present. This trend is not confined to beet. In chemotaxonomic studies on betalain distribution of the Caryophyllales, the correlation between betalamic acid and the presence of betaxanthins was found throughout the order (Steglich \& Strack, 1990). Given these trends, it is possible that betacyanin biosynthesis may use up free betalamic acid more effectively than betaxanthin synthesis. To test the co-ordinated up-regulation hypothesis of 
betalamic acid and cyclo-DOPA, data on cyclo-DOPA levels in relation to betalamic acid would be important.

Betalain biosynthesis in beet is largely controlled by two tightly linked loci, $R$ and $Y$. Dominant alleles at these loci are necessary for betalain pigment production (Goldman \& Austin, 2000). Wolyn and Gabelman (1989) also found that alleles at these loci determine the ratio of betacyanins to betaxanthins as well as controlling the appearance of betalains in the root and shoot. The $R$ locus was recently found to encode the cytochrome P450 (CYP76AD1) gene, responsible for cyclo-DOPA synthesis (Hatlestad et al., 2012). The $Y$ locus, although not fully characterised, possibly encodes the 4,5-DOPA extradiol dioxygenase (DOD) gene (Hatlestad, 2012), which converts DOPA to betalamic acid, essential for both betaxanthins and betacyanins. Negative regulation of $R$ and $Y$ alleles in certain tissues could be responsible for between and within organ patterning seen in this study. These two loci, however, do not explain the uneven distribution of specific betacyanin and betaxanthin compounds.

In the purple plant, the betacyanin aglycone betanidin was absent in leaves, being localised to stem, root and hypocotyl organs. Similar to anthocyanins, aglycones are less common than glycosylated betacyanins (Steglich \& Strack, 1990). Glycosylation of betacyanins occurs either after synthesis of cyclo-DOPA (Wyler et al., 1984) or after conjugation of betalamic acid with cyclo-DOPA (Sciuto et al., 1972). In stem, hypocotyl and root, the presence of the betacyanin aglycones might be due to reduced functioning of the glycosyltransferase (GT) or cleavage of glycosyl groups after glycosylation. Another compound pattern noted in purple leaves is a difference in the ratio of betanin isomers between younger and older leaf tissue. The betanin to isobetanin ratio was 91:9 in young leaves, and 71:29 in old leaves. The occurrence of betalainic isomers is thought be controlled at the betalamic acid stage. Gandía-Herrero and García-Carmona (2012) found that in nature, betalamic acid occurs $95 \%$ in the (S) form, and $5 \%$ in the (R) form. Their experiment demonstrated that DOD has a greater specific activity to the $(\mathrm{S})$ form. The ratio of 95:5 was similar to that found in young purple Swiss chard leaves, but not in older leaves. In the review by Stintzing and Carle (2004), deviation from this ratio was suggested to be caused by in vivo vacuolar $\mathrm{pH}$ changes from exposure to different environmental conditions, as several Amaranthus 
species were also found with varying betanin:isobetanin ratios in different plant parts (Cai et al., 2001).

In yellow Swiss chard organs, trace amounts of betanin were found in all organs except in leaves, where only decarboxy betanidins were detected. Decarboxylation of betacyanins can occur during pigment extraction through the use of ethanolic media or by heating degradation (Wybraniec, 2005; Wybraniec et al., 2006). However, it is unlikely that the extraction methods were the cause of decarboxy betanidin production. All samples analysed underwent the same extraction and purification processes at the same time, and decarboxy betanidins were not detected in purple tissues. It is, therefore, most likely that the degradation of the betacyanins in the yellow tissues occurred in vivo, before extraction. Given that decarboxylated betacyanins occurred only in the organs above the soil, it is possible that external environmental factors induced their production.

Different organs in the yellow plants also had differing betaxanthin profiles. In the beet cell culture study by Schliemann et al. (1999), all amino acids were accepted in the formation of betaxanthins, with no specificity to amino acid or stereoisomer. This suggests that the different tissues of Swiss chard may contain differing levels of amino acids available for betaxanthin formation. To get a clearer picture on the causes of compound changes in different Swiss chard organs, a larger study on distribution and production of pigments at different growth stages and environments would be required.

\subsubsection{Physiological roles of betalains in vegetative tissues}

Similarities in betalain pigmentation patterns within dermal, vascular and ground tissues in multiple organs may result from similar biological functions. It is also possible that the presence of pigmentation in some of these tissues have no function. However, the similarities of vegetative tissue pigmentation in distantly related betalain and anthocyanin species, suggest that betalain pigments perform various functional roles.

Theories regarding physiological function of betalains in vegetative organs are based upon those proposed for anthocyanic plants. As discussed briefly in Section 1.5, a number of functional theories for anthocyanins have been developed based on developmental timing, location of pigmentation and induction of biosynthesis by 
external factors. Only a few of these theories have been investigated for betalain-producing plants.

The common occurrence of betalains in dermal tissues suggests that these pigments may play a role in protecting photosynthetic apparati through screening out harmful light. Harmful levels of light may be prevented from reaching the photosynthetic tissue by the screening of red and purple pigments, thereby reducing photoinhibition and photodamage (Solovchenko \& Merzlyak, 2008). Betacyanic leaves showed enhanced photoprotection over similar leaves without betalain pigmentation by reducing transmittance of harmful light levels (Nakashima et al., 2011). In addition to screening out excess white light, betalains may help to reduce UV radiation reaching photosynthetic tissue. Betacyanins do not absorb UV light, though betaxanthin pigments have weak UV absorption ability (Gandía-Herrero et al., 2010; Gandía-Herrero et al., 2005b).

Alternative functional roles have been hypothesised for betalain pigments in dermal, vascular and ground tissues. Betalain pigments may mitigate the effects of biotic and abiotic stressors such as wounding, salt, cold and high light through their antioxidant capacity (Cai et al., 2003; Escribano et al., 1998). Betalain pigments present in the vacuoles are proposed to limit the damage caused by free radicals through scavenging excess $\mathrm{H}_{2} \mathrm{O}_{2}$ (Close \& Beadle, 2003). Although a role in scavenging $\mathrm{H}_{2} \mathrm{O}_{2}$ radicals in leaves was demonstrated for anthocyanins in vivo by Neill et al. (2002) and Kytridis and Manetas (2006), an in vivo antioxidant role has not yet been demonstrated for betalains. Another functional role suggested for betalains is assistance with salt sequestration. A number of betalainic plants grow in salty environments (Adams et al., 1998; Wang et $a l ., 2008)$. An issue that plants face under high saline conditions is salt toxicity. Leaves are particularly sensitive to high sodium concentrations (see review by Munns (2002)). One mechanism employed by salt tolerant plants, is the use of a vacuolar $\mathrm{Na}^{+} / \mathrm{H}^{+}$ antiporter, which sequesters $\mathrm{Na}^{+}$from the cytoplasm to store in the vacuole (Yokoi et al., 2002). Arabiodopsis mutants with overexpression of the $\mathrm{Na}^{+} / \mathrm{H}^{+}$antiporters on the tonoplast have demonstrated enhanced salt sequestration ability (Shi et al., 2002). In the betalainic halophyte Suaeda salsa, betacyanins may assist with vacuolar salt sequestration, as betacyanin levels have been positively correlated with enhanced $\mathrm{Na}^{+} / \mathrm{H}^{+}$antiporter activity (Wang et al., 2008). Another betalain-producing halophyte 
Mesembryanthemum crystallinum may utilise betalains in a similar salt management strategy. This plant has bladder cells on the tips of the leaves, which become red when the plant is exposed to stressful environmental conditions (Adams et al., 1998), a feature also seen on New Zealand spinach and ulluco organs (Figure 3.9A and 3.9B). Betalain pigmentation in the vacuoles of bladder cells might assist with the $\mathrm{Na}^{+}$ sequestration from the leaves.

Despite the numerous theories regarding betacyanin function, very little has been hypothesised for betaxanthins. The clear segregation of these compounds within and between organs for bougainvillea, ulluco and Swiss chard suggests these pigments may have different physiological roles. Future betalain physiology work should look into the possible protective benefits for each of these pigment types as well as in combination.

\subsubsection{Conclusion and future direction}

The similarities between betalain and anthocyanin localisation in tissues gives further support to the betalain replacement hypothesis. To elucidate how these pigments evolved as replacements, further information is needed on betalain biosynthesis. In the following research chapters, betalain storage, intracellular transport and biosynthetic regulation are investigated. 


\section{Chapter 4: Development of a betalain model system in Arabidopsis thaliana}

\subsection{Introduction}

Arabidopsis as a model system has a number of benefits (Hays, 2002). The plants are easy to grow and manage; they have a short life span (four weeks from sowing to maturity); they can self-pollinate for easy genetic crossing; they have high seed yield. Also, two key features are the availability of many characterised mutants, and its amenability to genetic transformation. Therefore, the production of betalains in Arabidopsis could be used to test physiology and biosynthesis questions in a controlled way.

A method for conferring betalain production to Arabidopsis thaliana was recently demonstrated by Harris et al. (2012) (Appendix 3). The plants have a betalain DOPA extradiol dioxygenase (DOD) gene from the fungal species, Amanita muscaria, inserted into their genome. This gene is responsible for the conversion of DOPA to betalamic acid which then spontaneously conjugates with cyclo-DOPA or amines/amino acids to form betacyanins and betaxanthins, respectively. In A. muscaria, DOD can also perform a 2,3-cleavage of DOPA to form the chromophore muscaflavin (Mueller et al., 1997b). When Portulaca grandiflora tissues were transformed with the DOD gene from A. muscaria, muscaflavin was detected alongside betalamic acid. It is unknown whether the A. muscaria DOD in Arabidopsis also produces muscaflavin along with betalains. The major pigments in A. muscaria, however, are all betalamic acid based (Lodge et al., 2013). Therefore, it is most likely that the Arabidopsis DOD transgeneics produce predominantly betaxanthins (when fed L-DOPA). The presence of these compounds in plant tissues is easily monitored by their autofluorescence under blue light (Harris et al., 2012).

One benefit of working with Arabidopsis as a model for betalain research is that Arabidopsis naturally produces anthocyanins, and have genetic lines that are available with constitutive anthocyanin pigmentation. Therefore, it is possible to make a plant 
that produces both betalains and anthocyanins in the same tissues. In this chapter the following Arabidopsis lines were analysed and genetic crosses made.

- Harris et al. (2012) produced six selfed $\left(\mathrm{T}_{2}\right)$ lines of DOD Arabidopsis. These lines were conferred with Basta antibiotic resistance for initial screening for the presence of the T-DNA containing the $35 S_{\text {pro }}$ :DOD transgene. For development of the lines used in this thesis, one of these lines was selected, DOD-6.

- For betalain transport analyses, two vacuolar transport mutants were selected and crossed with DOD-6. A number of Arabidopsis mutants have been identified for their involvement in anthocyanin/proanthocyanidin biosynthesis and transport. A phenotypic trait for many of these mutants is a pale seed coat which lent them the name transparent testa (Koornneef, 1990). The $t t$ mutants selected were $t t 12$ and $t t 19$. Both $t t 12$ and $t t 19$ are proanthocyanidin/anthocyanin transporter mutants; TT12 encodes a multidrug and toxic compound extrusion (MATE) transporter (Debeaujon et al., 2001) and TT19 encodes a glutathione $S$-transferase-like (GST-like) transporter (Kitamura et al., 2004).

- To create an Arabidopsis line that produces both anthocyanins and betalains, DOD-6 was crossed with the PAPl (Production of Anthocyanin Pigment 1) over-expression line, $35 S_{\text {pro }}: P A P 1$, made with the construct described by Rowan et al. (2009). Seven lines of $T_{2}$ seed were sourced from Andy Allan from Plant $\&$ Food Research, Auckland. One PAP1 line was selected for crossing with the DOD line, PAP1-5.

- I also investigated methods to confer betacyanins synthesis to the DOD-6 line. These included feeding cyclo-DOPA along with L-DOPA, and transforming the line with a construct containing a cDNA from Swiss chard which encodes cyclo-DOPA synthase (CYP76AD1).

\subsubsection{Aim}

In this chapter, DOD Arabidopsis was further developed as a model betalain pigment system. In particular, the focus of the model system development was to establish Arabidopsis lines for use in studies on the physiology of betalains in vegetative tissues, and to investigate mechanisms for betalain accumulation. 


\subsection{Materials and methods}

\subsubsection{Development of Arabidopsis DOD line}

\subsubsection{Selection of $\mathrm{T}_{2}$ DOD line with L-DOPA feeding}

Two inflorescences were harvested from each of six individual $\mathrm{T}_{2}$ lines of 35S:AmDOD Arabidopsis plants (ecotype Columbia, developed by Harris et al. (2012)) as well as from wild type Columbia Arabidopsis. The base of one inflorescence from each line was placed in $1.5 \mathrm{~mL}$ microcentrifuge tubes containing either water or $10 \mathrm{mM} \mathrm{L-DOPA}$ solution (Appendix 1.7.1) for $24 \mathrm{~h}$. Changes in colour and autofluorescence for L-DOPA and water fed inflorescences were compared using the Leica M205 FA stereomicroscope (Section 2.4.1).

\subsubsection{Genetic selection of $\mathrm{T}_{2}$ DOD line}

Expression of the $D O D$ transgene was analysed in the six $\mathrm{T}_{2} \mathrm{DOD}$ lines. A rosette leaf was sampled from each line, and from non-transgenic Columbia. Leaves were rinsed with water, to remove petals and soil, before immediate RNA extraction and cDNA synthesis (Section 2.6.2). PCR was performed (Section 2.6.4) with cDNA using the primer pairs for the DOD transgene (Genbank accession Y12886, DOD F and DOD R, Appendix 2), and the Arabidopsis Actin2 gene (Genbank accession AT3G18780, Act2S, and Act2AS, Appendix 2). PCR products from each DOD line were gel purified (Section 2.6.5.1), ligated into the pGEM-T Easy vector system (Section 2.6.6) and sent for sequencing by a commercial service (Section 2.6.9).

\subsubsection{Selfing and selection of DOD-6}

Line DOD-6 was selected for further use in this study. $\mathrm{T}_{3}$ seed was collected from selfed $T_{2}$ DOD-6 plants, sowed and plants were selected by a visual L-DOPA feeding screen (Section 4.2.1.1). Selfed $\mathrm{T}_{4}$ seed was then collected from plants with strong green autofluorescence.

\subsubsection{Feeding trials L-DOPA and cyclo-DOPA}

\subsubsection{Filter paper feeding}

A filter paper feeding method was tested for both seedlings and mature plants. For seedlings, Columbia and DOD-6 seeds were germinated directly on the filter paper in a 
Petri dish moistened with water. After one week, seedlings were fed with water or 10 mM L-DOPA (Figure 4.1A). For mature plants, Columbia and DOD-6 plants were grown in soil for 3 - 4 weeks, uprooted, rinsed with water and placed on filter paper in a Petri dish (Figure 4.1B). Filter paper was then wet with water, $10 \mathrm{mM}$ L-DOPA, $10 \mathrm{mM}$ cyclo-DOPA or $10 \mathrm{mM}$ L-DOPA $+10 \mathrm{mM}$ cyclo-DOPA with $20 \mathrm{mM}$ ascorbic acid solution (Appendix 1.7.1). Petri dishes were left on the bench overnight. Following the feeding of mature plants, an alkaline test (Section 2.9.2) was carried out to confirm presence of betacyanins. In addition, plants fed with both L-DOPA and cyclo-DOPA were freeze dried and sent to Nigel Joyce at Plant \& Food Reasearch in Lincoln for LC-MS analyses. Methods for pigment extraction and LC-MS analyses were the same as described in Sections 3.2.6 and 3.2.7.

\subsubsection{Hydroponic feeding}

Columbia and DOD-6 plants were grown in soil for two weeks following germination before being uprooted. Plant roots were rinsed of soil, wrapped in synthetic sponge and placed in microcentrifuge tubes, with the bottoms cut off, in hydroponic trays, which exposed the roots to hydroponic solution (Appendix 1.1.2) below (Figure 4.1C). Hydroponic solution was supplemented with water (control), $10 \mathrm{mM}$ cyclo-DOPA with $20 \mathrm{mM}$ ascorbic acid, or $10 \mathrm{mM}$ L-DOPA $+10 \mathrm{mM}$ cyclo-DOPA with $20 \mathrm{mM}$ ascorbic acid (Appendix 1.7.1). Hydroponic trays were left on the lab bench for three days.

\subsubsection{Filter tip feeding}

Columbia and DOD-6 seeds were surface sterilised and dark-stratified in water (Section 2.3.2). After two days at $4{ }^{\circ} \mathrm{C}, 4-5$ seeds were pipetted onto the top of each filter tip in culture tubs containing hydroponic solution (Appendix 1.1.2). After germination, seedlings were weeded to one per filter tip (Figure 4.1D). Five days following germination, filter tips were treated with hydroponic solution containing $1 \mathrm{mM}$ L-DOPA (Appendix 1.7.1). Over five days, filter tips were rinsed with fresh hydroponic solution daily, and treated again with the hydroponic solution containing $1 \mathrm{mM}$ L-DOPA.

\subsubsection{Liquid culture}

The method for liquid culture (Figure 4.1E) of Arabidopsis plants was modified from methods by Poustka et al. (2007). Columbia and DOD-6 seeds were surface sterilised 
and dark stratified (Section 2.3.2). After two days at $4{ }^{\circ} \mathrm{C}$, the seeds were aliquoted into culture tubs containing $10 \mathrm{~mL}$ water. Culture tubs were placed on rotary shaker in a room maintained at $22{ }^{\circ} \mathrm{C}$ with a $16 \mathrm{~h}$ photoperiod. After three days light exposure, seedlings were treated with $1 \mathrm{mM}$ L-DOPA solution or $1 \mathrm{mM}$ L-DOPA $+1 \mathrm{mM}$ cyclo-DOPA with $2 \mathrm{mM}$ ascorbic acid. The culture tub containing cyclo-DOPA was covered in tin foil to reduce the oxidation rate of cyclo-DOPA. Tubs were place back in the rotary shaker overnight.

\subsubsection{Creation of $D O D / C Y P 76 A D 1$ double transgenic}

\subsubsection{Isolation of CYP76AD1 from Swiss chard}

Tissue was harvested from fully expanded red Swiss chard leaves, frozen in liquid nitrogen and ground to a powder. RNA was extracted from $100 \mathrm{mg}$ of tissue (Section 2.6.2). Primers, BvCYP and BvCYP2, (Appendix 2) were designed to amplify the coding sequence for the betalain cytochrome P450 gene, CYP76AD1 (Genbank accession: HQ656023). Restriction sites BamHI and ClaI were added to the forward and reverse primers, respectively. cDNA synthesis followed methods in Section 2.6.2 with the following change. Specific priming was used for the cDNA synthesis by using the primers BvCYP and BvCYP2 instead of oligo-DT. This allowed for amplification of CYP76AD1 from low transcript levels. CYP76AD1 was isolated by PCR (Section 2.6.4), separated by gel electrophoresis (Section 2.6.3.1) and purified (Section 2.6.5.1).

\subsubsection{CYP76AD1 over-expression construct creation}

Purified CYP76AD1 was ligated (Section 2.6.6) into BamHI and ClaI sites of pART7 (Gleave, 1992) which placed the CYP76AD1 gene under the control of the CaMV35S promoter and added the octopine synthase $(O C S)$ terminator. The pKEC1 (35 $S_{\text {pro }}:$ CYP76AD1, Appendix 4A) construct was digested with NotI and subcloned into the NotI site of pART27 binary vector (Gleave, 1992). This generated the plasmid pKEC2 (35 $S_{\text {pro }}:$ CYP76AD1, Appendix 4B), which was transformed into Agrobacterium tumefaciens strain GV3101 by electroportation (Section 2.7.5). A. tumefaciens cultures harbouring the pKEC2 binary vector were used for Argrobacterium-mediated transformations of DOD-6 Arabidopsis. 


\subsubsection{Arabidopsis transformation}

Forty DOD-6 plants were grown to maturity. After plants produced a primary inflorescence stalk, the stalk was removed to enhance development of secondary inflorescences. This allowed for the greatest number of plant inflorescences to be at a stage just prior to anthesis for transformations, as recommended by Clough and Bent (1998) and Chung et al. (2000). Arabidopsis plants were transformed with the A. tumefaciens line harbouring the binary vector pKEC2 (Appendix 4B) following the methods in Section 2.8.2. After a one week recovery period, the dipping of inflorescences was repeated. The inflorescence dipping was repeated for a third time one week later. Mature siliques were harvested and dried for 3 weeks before seeds were collected and stored in the dark at $4{ }^{\circ} \mathrm{C}$.

\subsubsection{Selection of PAP1 transgene line}

Selection for the presence of the $P A P 1$ transgene was carried out visually on seven $\mathrm{T}_{2}$ 35S pro:PAP1 Arabidopsis lines in ecotype Columbia. Line PAP1-5 was selected for further analyses in this study. As visually assessed, this line had the most consistent anthocyanin pigmentation in the vegetative tissues. $T_{3}$ PAP1-5 seed was sown and green seedlings were weeded out, keeping only the intensely purple-coloured plants. These plants were then selfed and $\mathrm{T}_{4}$ seed collected.

\subsubsection{Arabidopsis crosses: DOD-6 with $t t 12, t t 19$ and PAP1-5}

\subsubsection{Origin of $t t$ seed}

Seed of transgenic T-DNA Arabidopsis mutants was sourced from the Arabidopsis Biological Resource Center (ABRC). T-DNA insertional mutations were made by the Salk Institute in Columbia using the $\mathrm{T}_{\mathrm{i}}$ plasmid of Agrobacterium (O'Malley et al., 2007). The insertion sites of T-DNA were in gene-coding regions, disrupting gene function. The mutant lines sourced from ABRC were, tt12 (ABRC: CS339467; Kleinboelting et al. (2012)) and tt19 (ABRC: SALK_068448.42.40.x; Alonso et al. (2003)). Both $t t 12$ and $t t 19$ are proanthocyanidin/anthocyanin transporter mutants; $t t 12$ - a MATE transporter mutant (Debeaujon et al., 2001) and tt19 - a GST transporter mutant (Kitamura et al., 2004). Both mutant lines were homozygous for T-DNA inserts in exon regions of the genes. 


\subsubsection{Crossing of $t t 12, t t 19$ and PAP1-5 with DOD-6}

Arabidopsis plants were grown until the plants bolted (around 4 - 5 weeks). As these plants are highly self-pollinating, crosses were carried out on immature flowers. Using fine tweezers, the sepals, petals and immature stamen were removed, leaving an intact pistil. To complete the cross, the exposed pistil was brushed gently with a mature flower of another Arabidopsis line. Reciprocal crossings were completed for each of the following crosses, DOD-6 x tt12, DOD-6 x tt19 and DOD-6 x PAP1-5. Flowers surrounding the crossed flowers were removed and the inflorescence was marked with string. The meristematic tip of the inflorescence was also crushed to inhibit growth of the stem. Siliques were harvested when they had matured and seeds were dried. The seed of reciprocal crossings were combined.

\subsubsection{Genotyping DOD-6 x $t t 12$}

PCR screening (Section 2.6.4) was used to genotype the cross DOD-6 x $t$ t12. gDNA extracted (Section 2.6.1) from $\mathrm{F}_{2}$ plant tissues was used in multiple PCR reactions. The initial PCR screen was for the DOD gene using primers DOD F and DOD R (Appendix 2). Plants positive for $D O D$ were then screened for the T-DNA insert using primers SALK insert F and SALK insert R (Appendix 2), which amplified the left border region of the T-DNA insert. The genotyping protocol recommended by the Salk Institute (Leicht \& Cheng, 2009) was used to distinguish between homozygotes and heterozygotes for plants with positive T-DNA insertions (Figure 4.2). Genotyping primers, tt12LP and tt12RP (Appendix 2), were designed on the SIGnAL T-DNA Primer Design page on the Salk website (http://signal.salk.edu/tdnaprimers.2.html; Salk Institute for Biological Studies). These primers were the left and right genomic primers, respectively, which amplified part of the wild type TT12 gene (1 237 bp product). The third primer used in the PCR reaction was LBb1 (Appendix 2), a primer designed by the Salk institute which binds to the left border region of the T-DNA insert. LBb1 in combination with tt12RP amplified part of the wild type gene along with part of the T-DNA insert (539 bp product). Genotyping PCR used all three primers (tt12LP, tt12RP and LBb1) in a single reaction. Homozygous lines for wild type or T-DNA insert had a single band at either 1237 bp or $539 \mathrm{bp}$, respectively. Heterozygous lines had two bands, one at $1237 \mathrm{bp}$ and one at $539 \mathrm{bp}$. All plants identified to contain DOD and were 
homozygous for the $t t 12$ T-DNA insert were selfed, and seed pooled. See Figure 4.3 for a schematic diagram of the DOD- 6 x $t t 12$ crossing.

\subsubsection{Genotyping DOD x $t$ t 19 with phenotyping screen}

Genotyping DOD-6 x tt19 lines used the two initial PCR screens as used for genotyping DOD-6 $x$ tt12 crosses (Section 4.2.5.3). gDNA extracted from $F_{2}$ plants were screened for $D O D$ and whether they contained the T-DNA insert. Plants positive for both the $D O D$ and T-DNA inserts were then screened with phenotyping. An anthocyanin induction method adapted from Poustka et al. (2007) was used as a phenotyping screen to identify homozygotes for the T-DNA insert allele. Seeds from each DOD x tt19 line as well as DOD-6 seeds were surface sterilised (Section 2.3.2) and grown in liquid culture (Section 4.2.2.4) using water or $3 \%(\mathrm{w} / \mathrm{v})$ sucrose solution. After $3 \mathrm{~d}$ in the light, $100 \mu \mathrm{M}$ naringenin (100 mM stock in EtOH) was added to seedlings in the sucrose solution. The next day seedlings were observed under the Leica dissecting microscope (Section 2.4.1) for the extent of anthocyanin accumulation. All plants identified to contain $D O D$ and were homozygous for the $t t 19$ T-DNA insert were selfed, and seed pooled. See Figure 4.3 for a schematic diagram of the DOD-6 x tt19 crossing.

\subsubsection{Selection of DOD-6 x PAP1-5}

$\mathrm{F}_{1}$ seedlings containing $P A P 1$ were screened visually by weeding out green-leafed plants in favour of purple seedlings, as in Section 4.2.4. The screening for $D O D$ involved feeding either leaves or inflorescences $10 \mathrm{mM} \mathrm{L-DOPA}$ solution overnight and selecting plants with strong green autofluorescence under blue light using the Leica M205 FA stereomicroscope (Section 2.4.1). Selected plants were selfed, and $F_{2}$ seed was collected. $F_{2}$ seed was sown and selected as previously, selfed and $F_{3}$ seed was collected. All seed from selected plants at each selfing was pooled. 


\section{Filter paper}
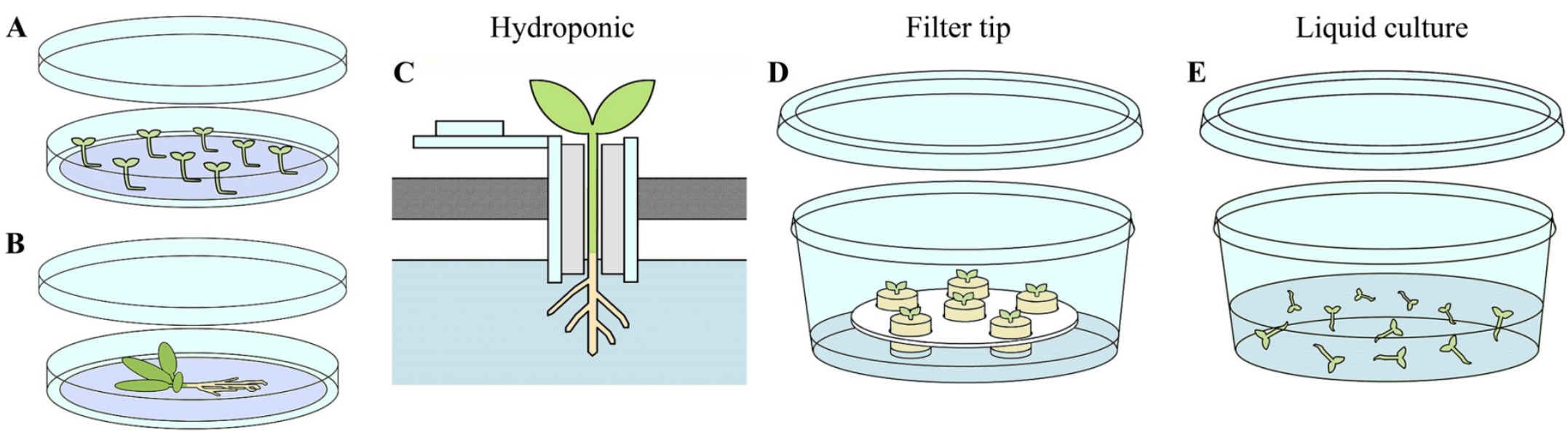

Figure 4.1 Illustration of the four feeding methods trialled for L-DOPA and cyclo-DOPA to DOD-6 plants: filter paper feeding of (A) seedlings and (B) mature plants, (C) hydroponic feeding to 3 - 4 week old plants, (D) filter tip feeding to seedlings, (E) liquid culture feeding to seedlings. 

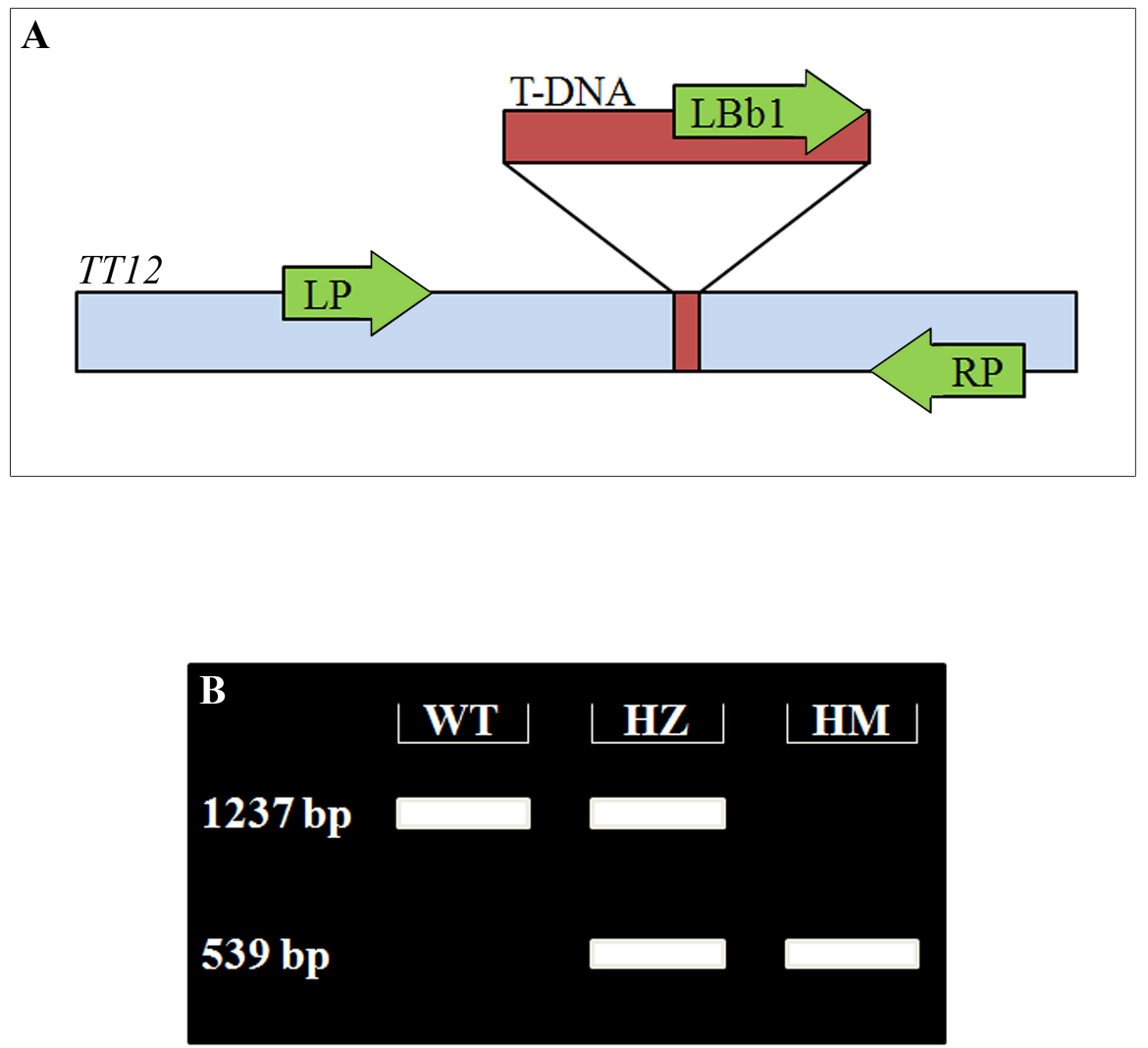

Figure 4.2 Diagrams of Salk Institute PCR screening methods used for genotyping DOD-6 $x$ tt12. (A) Schematic view, not to scale, of the T-DNA insert in the TT12 gene with positions of primers LP (left genomic primer), LBb1 (left border primer) and RP (right genomic primer). (B) Schematic of expected PCR products on gel electrophoresis of reactions using the three primers LP, LBb1 and RP. Wild type (WT) would have a single band at $1237 \mathrm{bp}$ (the size of band without the insert), a heterozygote (HZ) would have bands at $1237 \mathrm{bp}$ and $539 \mathrm{bp}$ (for the two alleles) and a homozygote (HM) would have a single band at $539 \mathrm{bp}$ (the distance between the LBb1 and RP primers). Diagrams were adapted from those on the Salk primer design page (http://signal.salk.edu/tdnaprimers.2.html). 
DOD-6

tt mutant

$\begin{array}{llllll}\text { [1] TT/TT DOD/DOD } & \mathbf{x} \quad t t / t t-/-\quad \text { Parent lines } \\ & \downarrow\end{array}$

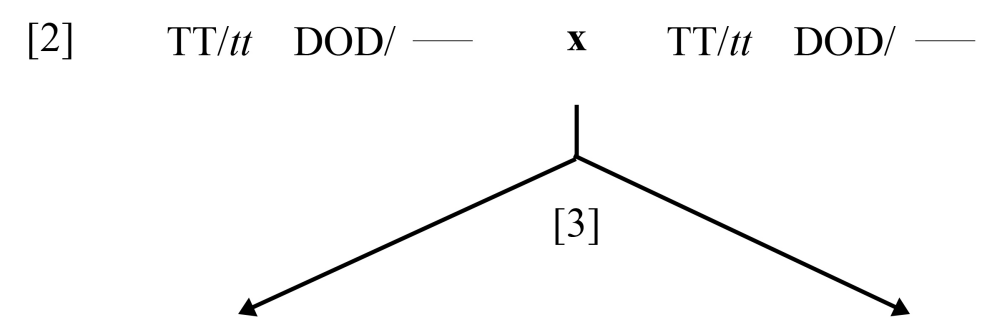

F1 generation

$\stackrel{\infty}{\sim}$

[4] $t t / t t \quad \mathrm{DOD} /-\quad \mathbf{x} \quad t t / t t \quad \mathrm{DOD} /-$

$t t / t t \quad$ DOD/DOD $\quad \mathbf{x} \quad t t / t t \quad$ DOD/DOD

F2 generation

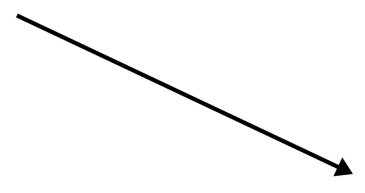

[5]

Pooled seed

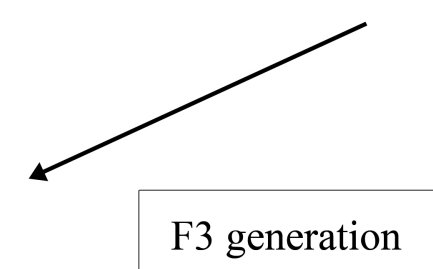

Figure 4.3 Steps carried out in the genetic crossing of DOD-6 with the $t$ mutants: $t t 12$ and $t t 19$. [1] Initial crossing of DOD-6 (TT/TT DOD/DOD) with $t t 12$ or $t t 19(t t / t t-/-)$. [2] Selfing of the offspring, TT/tt DOD/-. [3] Selection of homozygotes for the $t t$ T-DNA insert and the presence of $D O D(t t / t t \mathrm{DOD} /-$ or $t t / t t$ DOD/DOD) using genotyping/phenotyping methods. [4] Selfing of selected offspring. [5] Seed pooled from selected offspring. 


\subsection{Results}

\subsubsection{Selection of DOD line}

$D O D$ expression in six independent transgenic lines was assessed (Appendix 5A). DOD lines 6 and 7 had the clearest bands from the PCR reaction, indicating that these lines definitely had the $D O D$ gene. These two lines were then assessed for their ability to accumulate betaxanthins by L-DOPA feeding, similar to Figure 4.4. From these analyses, line DOD-6 was chosen for future experiments as it had the strongest visible pigment accumulation. Subsequent generations of DOD-6 were screened effectively using L-DOPA feeding.

\subsubsection{L-DOPA and cyclo-DOPA feeding trials}

Feeding L-DOPA and cyclo-DOPA to DOD-6 was trialled using four methods: filter paper, a hydroponic system, filter tips, and liquid cultures (Figure 4.1). These different feeding methods gave varied betaxanthin and betacyanin accumulating ability, and they differed in their effect on plant health. Wild type Columbia Arabidopsis plants were used as negative betalain-accumulating controls in all of these feeding methods and were found not to visually produce any betalains, based on visual assessments of colour and autofluorescence.

\subsubsection{Filter paper feeding}

Filter paper feeding with a L-DOPA solution (Figure 4.1A and 4.1B), used either germinated seedlings, or 3 - 4 week old plants. Seedlings responded well to this feeding method, with fast pigment accumulation (Figure 4.5A). However, these seedlings were susceptible to fungal infection and tissue damage from the constant exposure to a wet surface. Whole plants were slower accumulators of betaxanthin pigments than seedlings (Figure 4.5B), and were also affected by tissue damage where the plant was in constant contact with wet filter paper. Addition of cyclo-DOPA to the L-DOPA solution to induce betacyanin production resulted in fast oxidation of cyclo-DOPA to a red/black compound (Figure 4.6A). If the feeding solution also contained ascorbic acid, oxidation of cyclo-DOPA to this red/black compound was reduced (Figure 4.6A and 4.6B). Whole plants fed with both L-DOPA and cyclo-DOPA produced both betaxanthins and betacyanins (Figure 4.6A). Betaxanthins were confirmed in these plants by their green autofluorescence under blue light. Presence of betacyanins was confirmed in two ways. 
Firstly, the solution surrounding L-DOPA and cyclo-DOPA fed plants was purple (Figure 4.6B), and when placed in an alkaline solution the solution turned yellow, indicating betacyanins (Figure 4.6C). Secondly, LC-MS analyses on the pigment extracts from these plants indicated that the plants contained betaxanthins and betacyanins, in addition to anthocyanins (Figure 4.6D). These LC-MS results indicate that DOD in Arabidopsis likely performed the 4,5-cleavage of DOPA, as the compounds identified are betalamic acid based. However, future HPLC pigment analyses should incorporate an absorbance measurement at $405 \mathrm{~nm}$ to check for the muscaflavin peak. Muscaflavin and betalamic acid have different HPLC retention times, betalamic acid comes out a few minutes before muscaflavin (Terradas \& Wyler, 1991). If L-DOPA fed DOD-6 samples were run alongside a beetroot standard, HPLC traces could be compared. The beetroot standard would have single peak for betalamic acid, and if DOD in DOD-6 performs the 2,3-cleavage of DOPA, second peak for muscaflavin would be present in addition to betalamic acid.

\subsubsection{Hydroponic feeding}

Three to four week old plants were uprooted from soil and washed before being placed in a hydroponic system (Figure 4.1C). After three days in the hydroponic system, the plants in hydroponic solution with water added appeared slightly yellowed, but healthy (Figure 4.7A and 4.7B). The addition of cyclo-DOPA with ascorbic acid (Figure 4.7C and 4.7D) and L-DOPA + cyclo-DOPA with ascorbic acid (Figure 4.7E and 4.7F) to the hydroponic solution resulted in stressed and withered plants, for both Columbia and DOD-6 plants. Despite appearing stressed, DOD-6 plants showed visual accumulation of betalain pigments with the L-DOPA + cyclo-DOPA treatment, under white and blue light. Attempts to reduce the acidity of solution by buffering the hydroponic solution resulted in faster oxidation of cyclo-DOPA to dopachrome (data not shown).

\subsubsection{Filter tip feeding}

Seeds were germinated on top of the filter tips and grown for several days before addition of L-DOPA (Figure 4.1D). L-DOPA added directly to the filter tip oxidised overnight. Addition of ascorbic acid was previously trialled to reduce L-DOPA oxidation, but resulted in poor seedling germination and growth (results not shown), so ascorbic acid was not used. Feeding of DOPA + cyclo-DOPA feeding was also attempted, but this compound oxidised rapidly. The rinsing of the filter tips between 
L-DOPA additions reduced oxidised L-DOPA build up. Sufficient L-DOPA reached the growing tissues to produce betaxanthins after $24 \mathrm{~h}$ in DOD-6 seedlings (Figure 4.8). Betaxanthin concentration peaked on the fourth day and reduced on the fifth day as estimated visually by autofluorescence levels.

\subsubsection{Liquid culture feeding}

Seedlings were germinated in solution before the addition of L-DOPA (Figure 4.1E). This method resulted in the fastest pigment accumulation of the methods trialled and gave good germination rates of DOD-6 (Figure 4.9A). After $24 \mathrm{~h}$, seedlings fed with L-DOPA had strong pigment accumulation and autofluorescence (Figure 4.9B). The addition of both L-DOPA and cyclo-DOPA to the solution reduced betaxanthin accumulation and caused strong oxidation of both compounds around the roots (Figure 4.9C).

\subsubsection{Creation of $D O D / C Y P 76 A D 1$ double transgenic plants}

Attempts to produce purple DOD-6 plants by feeding cyclo-DOPA along with L-DOPA resulted in inefficient betacyanin accumulation (Section 4.3.2). To work around this problem, DOD-6 plants were transformed with the cDNA for the cytochrome P450 betalain biosynthetic enzyme (CYP76AD1) isolated from Swiss chard (Appendix 5B). As the DOD-6 line already harboured a pART27 vector with Basta selection, a pART27 vector with Kanamycin antibiotic resistance was inserted for future selection of CYP76AD1. During vector creation with CYP76AD1, the functionality of the CYP76AD1 gene was tested using transient gene expression in yellow Swiss chard. The shooting of construct pKEC1, containing $35 S_{\text {pro }}$ : CYP76AD1, into yellow Swiss chard petioles turned the transformed cells red (Figure 6.15), indicating that the transgene was functional. The transformation experiment was then conducted and seed harvested. Selection for transformed plants has not yet been done.

\subsubsection{Selection of PAP1-5 and DOD-6 x PAP1-5 plants}

Visual methods were used to select $P A P 1$ and $D O D$ transgenes in PAP1-5 and DOD-6 $\mathrm{x}$ PAP1-5 lines. Plants containing the PAP1 transgene were identified by selecting the plants with the strongest, consistent purple pigmentation throughout the plant (Figure 4.10A). DOD-6 x PAP1-5 plants containing DOD were selected using a L-DOPA feeding screen (Figure 4.10B). The autofluorescence of betaxanthins was obvious under 
blue light. Plants containing $P A P 1$ were found to grow slower and reach maturity later than DOD-6 plants (Figure 4.11).

\subsubsection{DOD-6 $\times t t$ crosses}

\subsubsection{DOD-6 x tt12}

The identification of plants containing DOD-6 in the $t t 12$ background, from the DOD-6 $\mathrm{x} t t 12$ cross, used three PCR reactions. Initial PCR reactions for $D O D$ and the $t t \mathrm{~T}$-DNA insert (Appendix 5C) allowed the offspring number to be narrowed down before screening for the homozygotes of the $t t$ T-DNA allele. Three $t t$ T-DNA homozygotes were successfully screened from the heterozygotes using the Salk screening methods (Appendix 5D).

\subsubsection{DOD-6 x tt19}

The cross DOD-6 x $t t 19$ was initially screened for $D O D$ and the $t t$ T-DNA inserts in the same way as Section 4.3.5.1. DOD-6 x tt19 plants positive for both $D O D$ and the T-DNA insert were initially screened using Salk designed primers. These primers amplified a large number of unspecific products so were not used in determination of the cross. Instead, homozygotes were screened from heterozygotes using a phenotype screen. Anthocyanin production was induced in seedlings with the feeding of naringenin (Figure 4.12). When compared with DOD-6 seedlings (Figure 4.12B), both $t$ t19 (Figure 4.12C) and DOD-6 x tt19 (Figure 4.12D) plants that were homozygotic for the $t t$ T-DNA insertion allele had reduced anthocyanin accumulation in the cotyledons. 


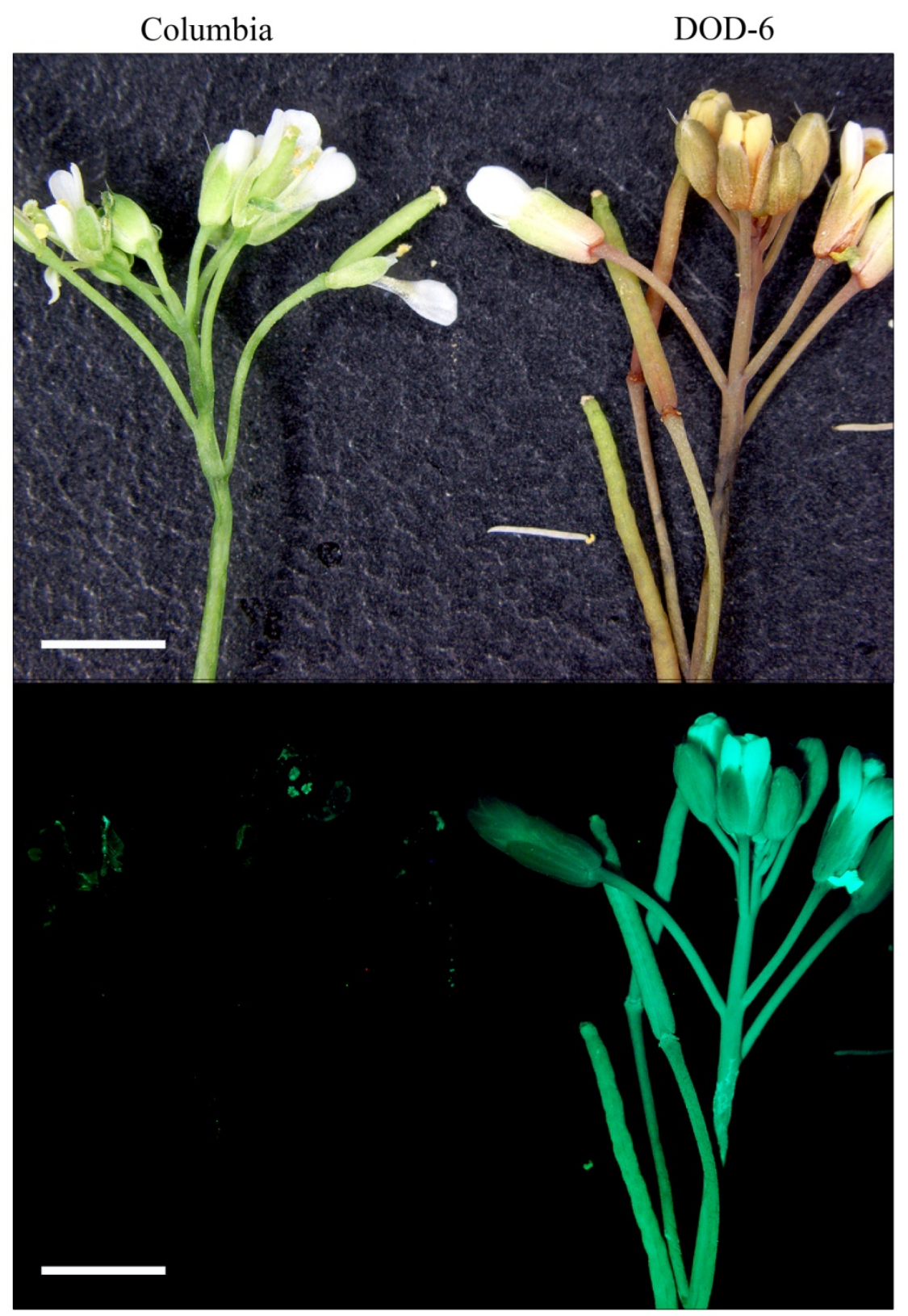

Figure 4.4 Columbia and DOD-6 inflorescences fed with $10 \mathrm{mM}$ L-DOPA for DOD line selection. Inflorescences were examined under white (top) and blue (bottom) light for changes in pigmentation and autofluorescence, respectively. Scale bars $=6 \mathrm{~mm}$. Figure adapted from Harris et al. (2012). 

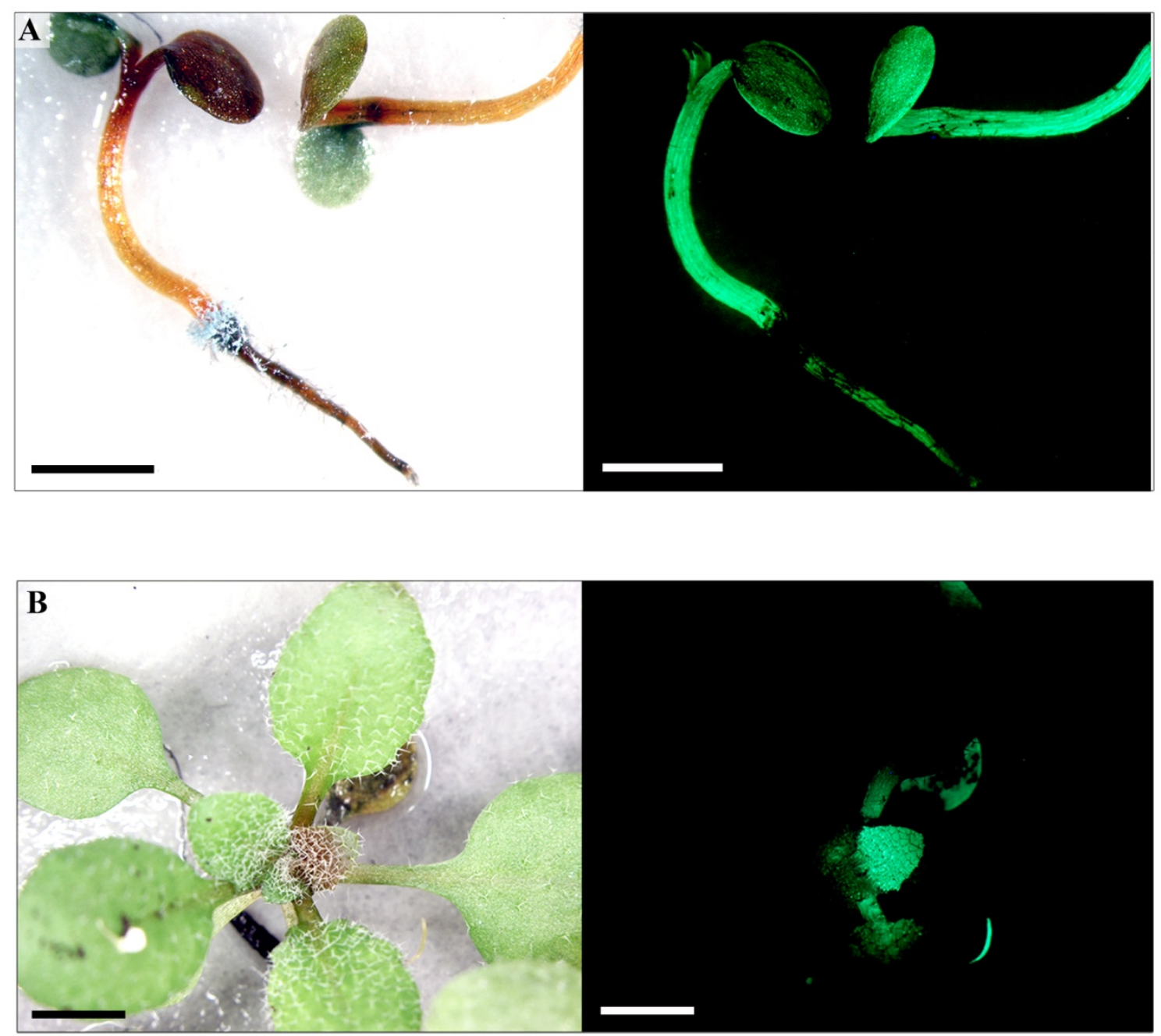

Figure 4.5 White light and blue light micrographs of filter paper feeding of $10 \mathrm{mM}$ L-DOPA to (A) DOD-6 seedlings and (B) mature plants. Scale bars: $\mathbf{A}=2 \mathrm{~mm}, \mathbf{B}=$ $5 \mathrm{~mm}$. 

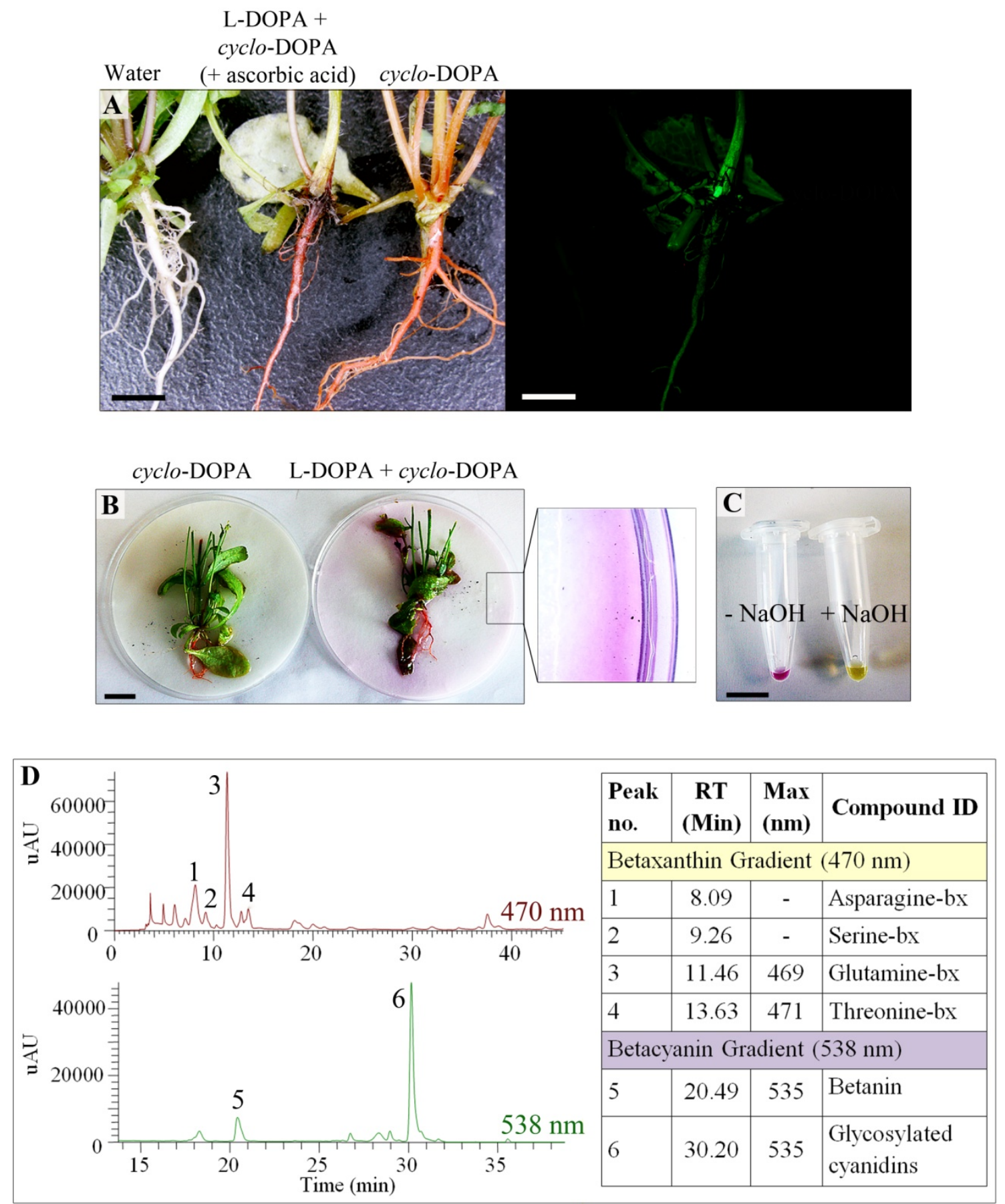

Figure 4.6 DOD-6 plants fed with L-DOPA + cyclo-DOPA using the filter paper method. (A) Plants fed with water (left), $10 \mathrm{mM}$ L-DOPA + cyclo-DOPA with $20 \mathrm{mM}$ ascorbic acid (middle), or $10 \mathrm{mM}$ cyclo-DOPA only (right) and their autofluorescence under blue light. (B) Solution colour of cyclo-DOPA only (left), or L-DOPA + cyclo-DOPA (right). (C) Alkaline test on solution from the L-DOPA + cyclo-DOPA plate in B. (D) LC-MS analyses on L-DOPA + cyclo-DOPA fed DOD-6 plants measured at $470 \mathrm{~nm}$ and $538 \mathrm{~nm}$, compound identities in table on right. Scale bars: $\mathbf{A}=$ $5 \mathrm{~mm}, \mathbf{B}$ and $\mathbf{C}=10 \mathrm{~mm}$. 

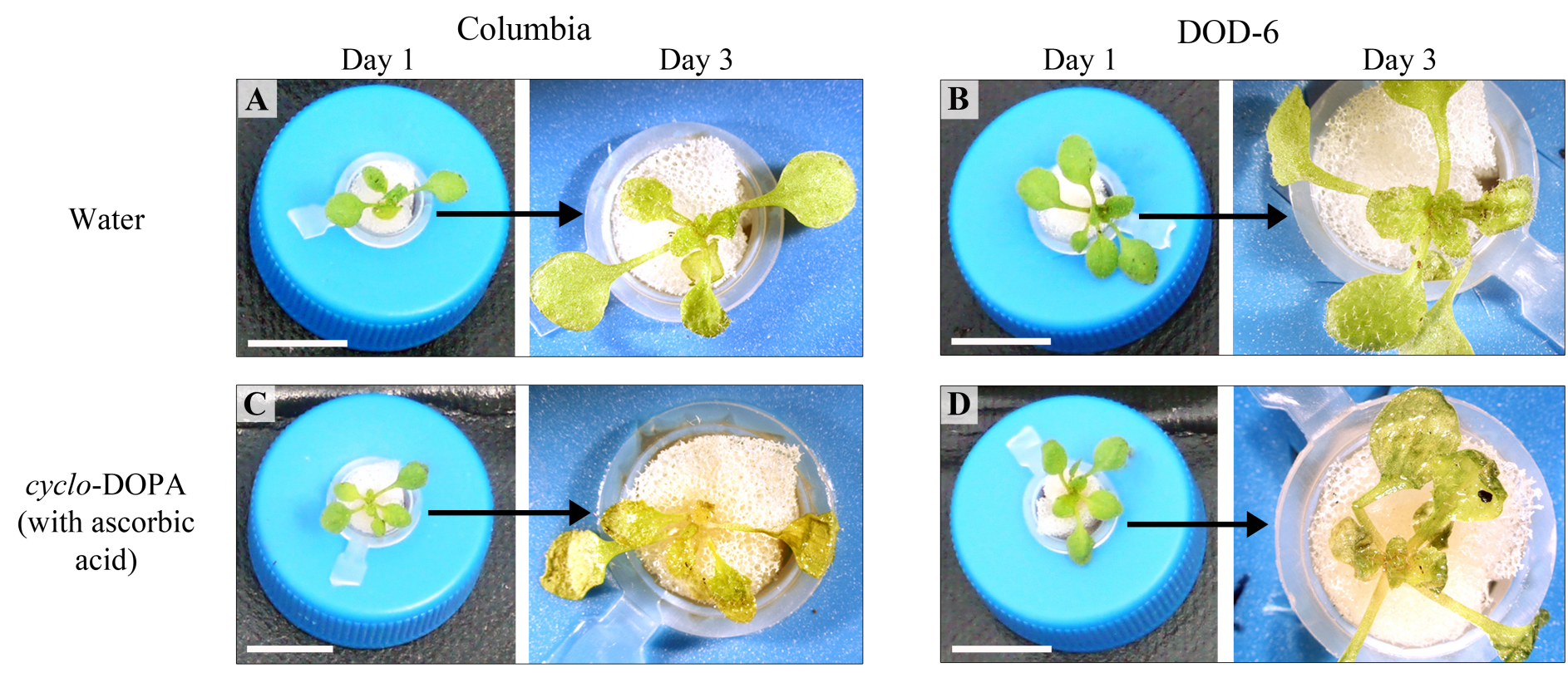

Blue light
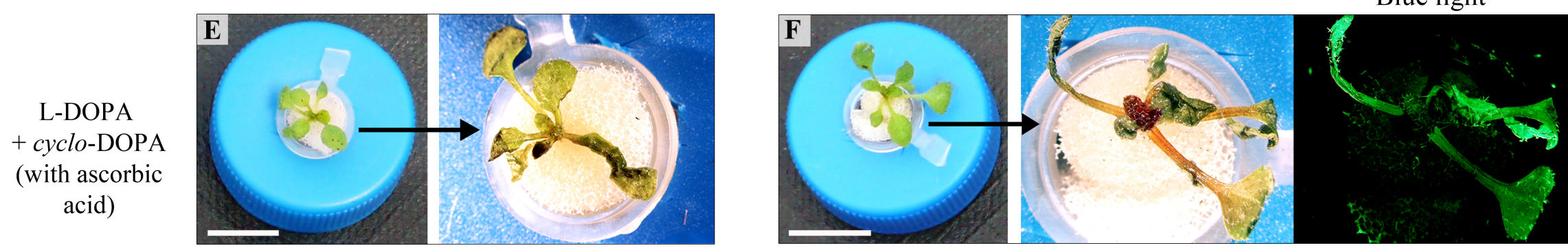

Figure 4.7 Plants in hydroponic solution fed with additional water, $1 \mathrm{mM}$ cyclo-DOPA only, or $1 \mathrm{mM}$ L-DOPA $+1 \mathrm{mM}$ cyclo-DOPA (with $2 \mathrm{mM}$ ascorbic acid), showing Columbia and DOD-6 plants on Day 1 and Day 3. Autofluorescence shown in panel $\mathbf{F}$ is from monitoring under blue light. Scale bars $=10 \mathrm{~mm}$. 


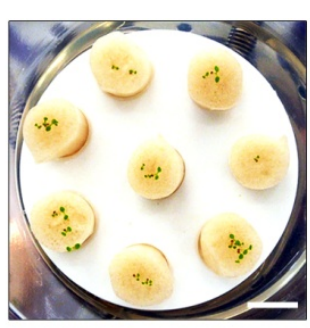

Day 1

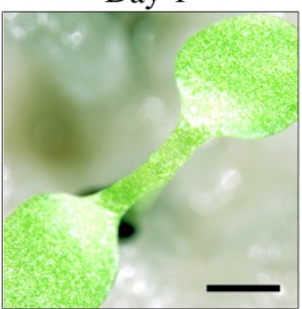

Columbia

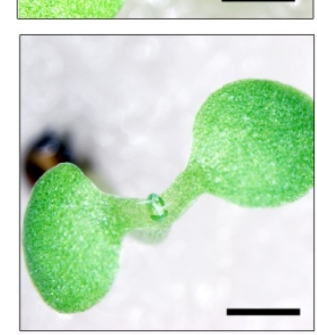

-
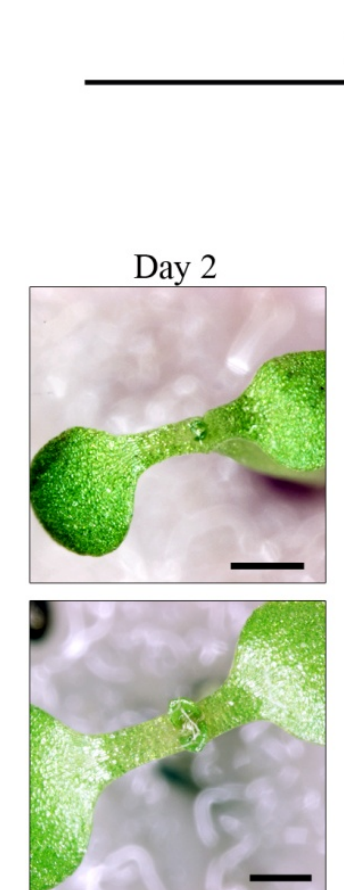

4.
L-DOPA feeding

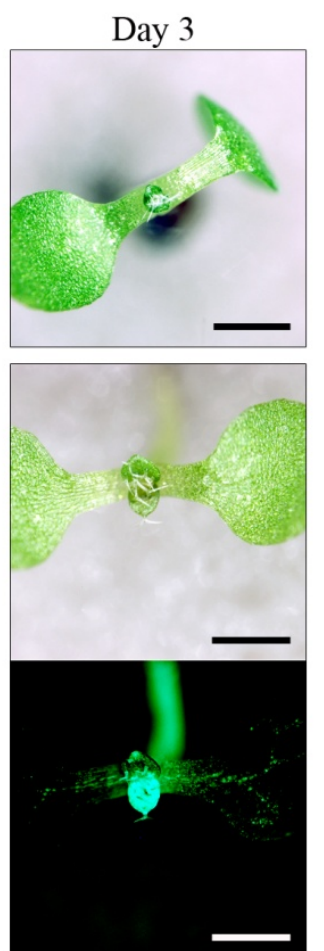

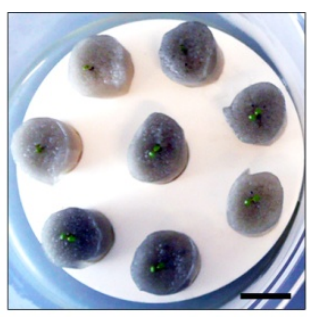

Day 5
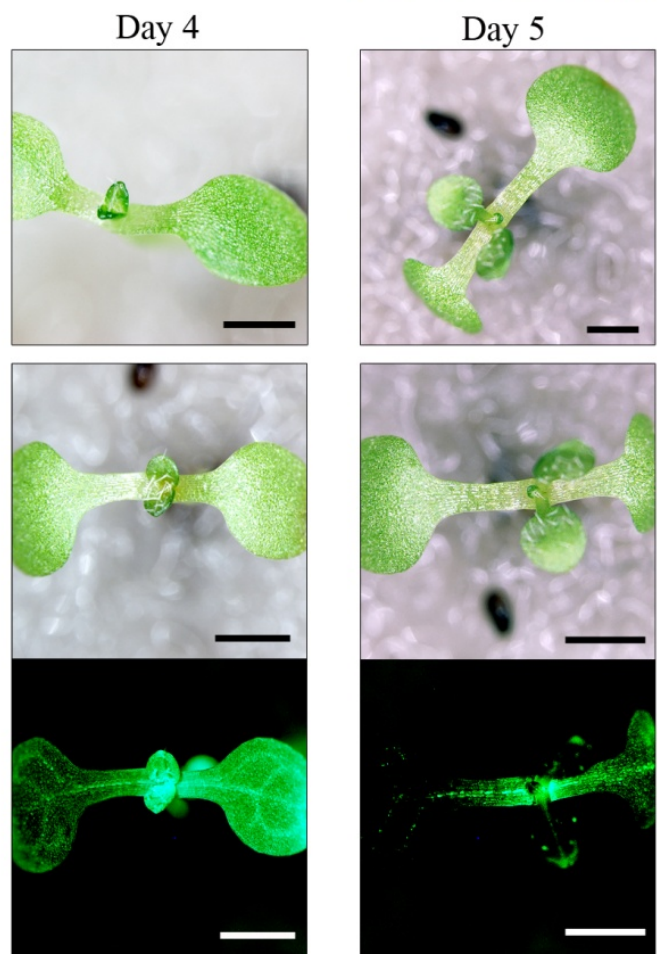

Figure 4.8 Filter tip feeding of $1 \mathrm{mM}$ L-DOPA to Columbia and DOD-6 seedlings over five days, photographed under white and blue light. Scale bars: culture tubs $=10 \mathrm{~mm}$, individual seedling images $=0.5 \mathrm{~mm}$. 

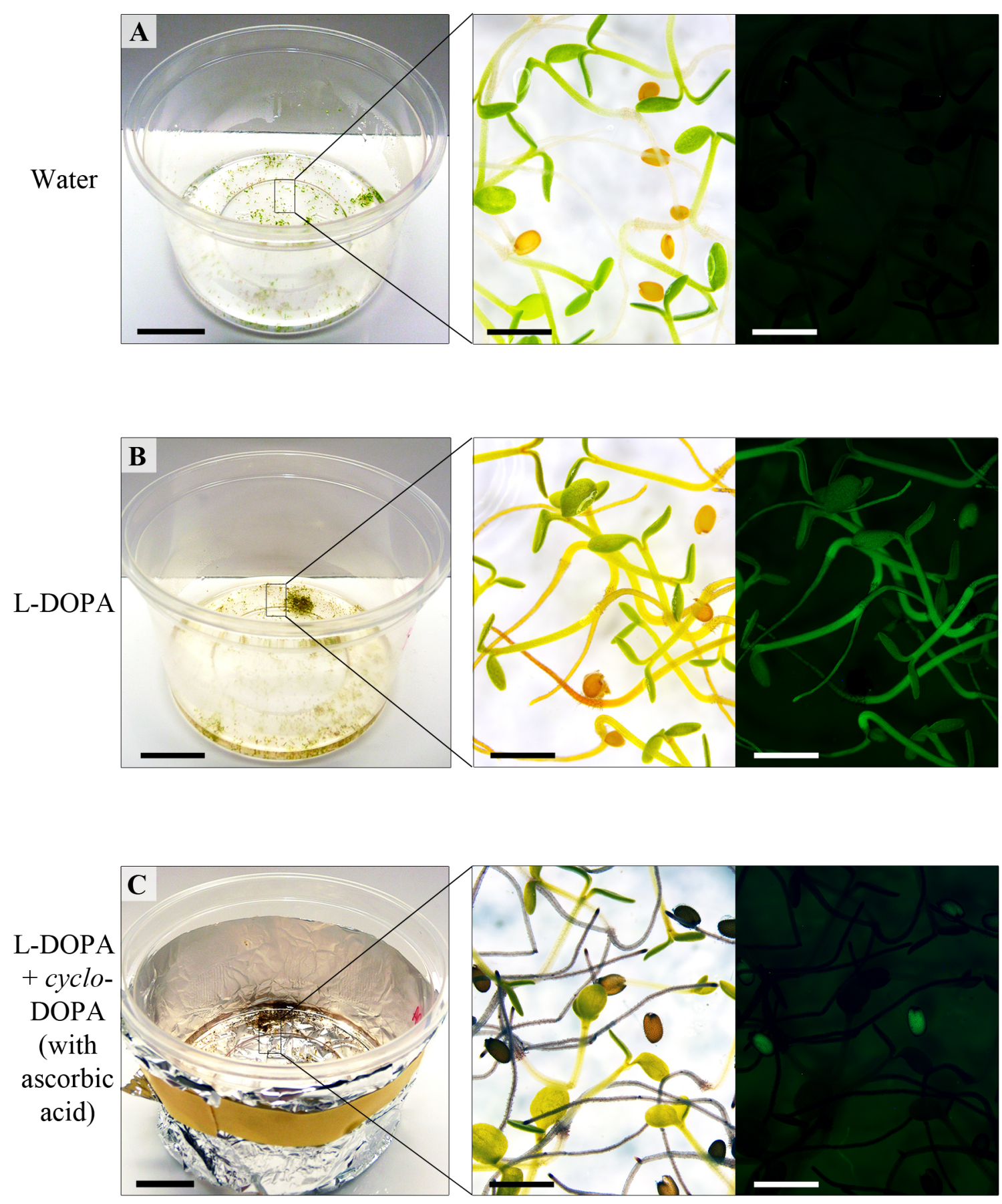

Figure 4.9 Liquid culture feeding of DOD-6 seedlings with (A) water, (B) $1 \mathrm{mM}$ L-DOPA, and (C) $1 \mathrm{mM}$ L-DOPA + cyclo-DOPA with $2 \mathrm{mM}$ ascorbic acid. Scale bars: A, B and $\mathbf{C}=20 \mathrm{~mm}$, inserts $=1 \mathrm{~mm}$. 

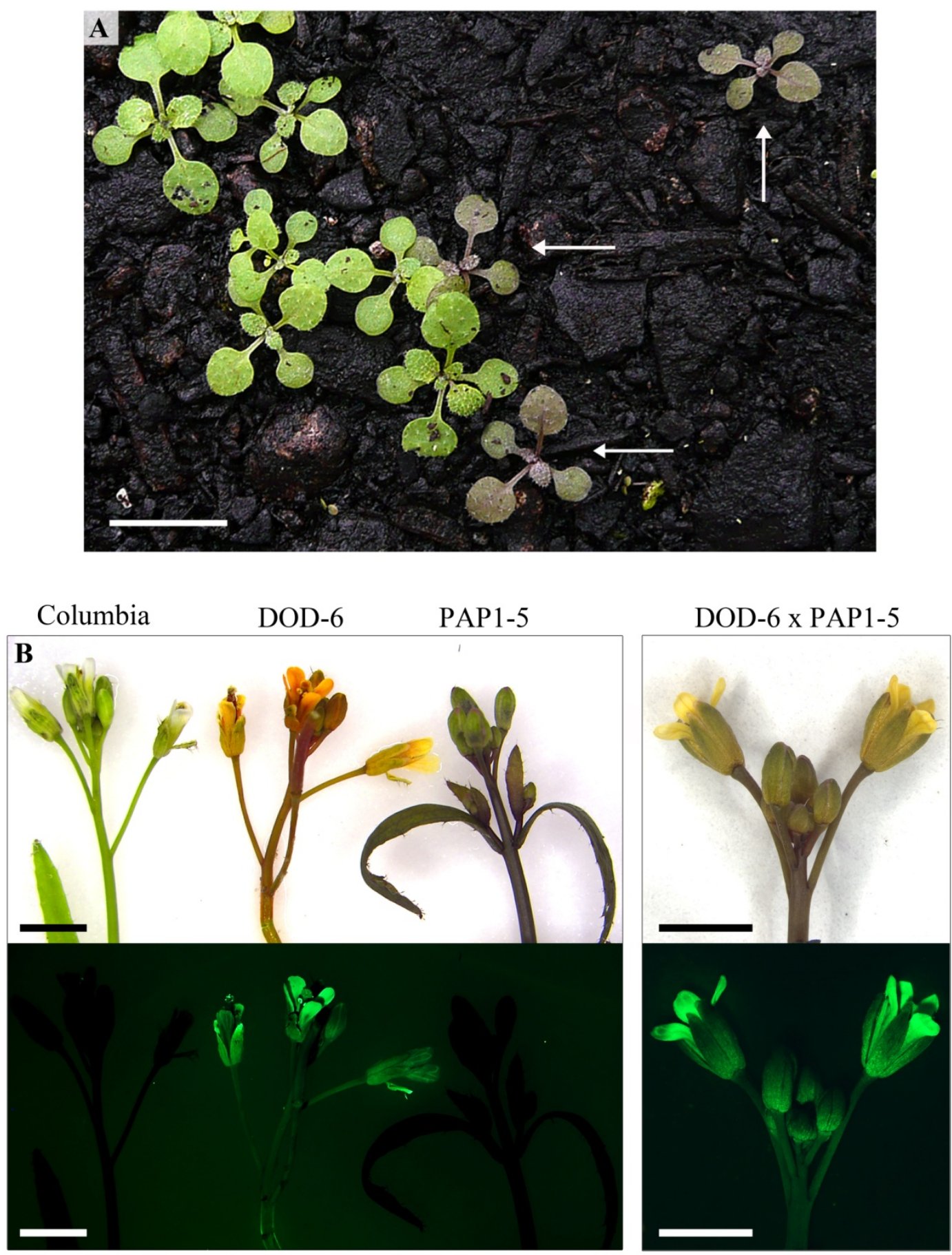

Figure 4.10 Visual selection of DOD-6 x PAP1-5 plants. (A) PAP1 selected by purple vegetative tissues, plants selected as indicated by arrows. (B) DOD selected by inflorescences fed with $10 \mathrm{mM}$ L-DOPA and examination under white and blue light. From left to right: Columbia, DOD-6, PAP1-5 and DOD-6 x PAP1-5. Scale bars: $\mathbf{A}=$ $8 \mathrm{~mm}, \mathbf{B}=5 \mathrm{~mm}$. 

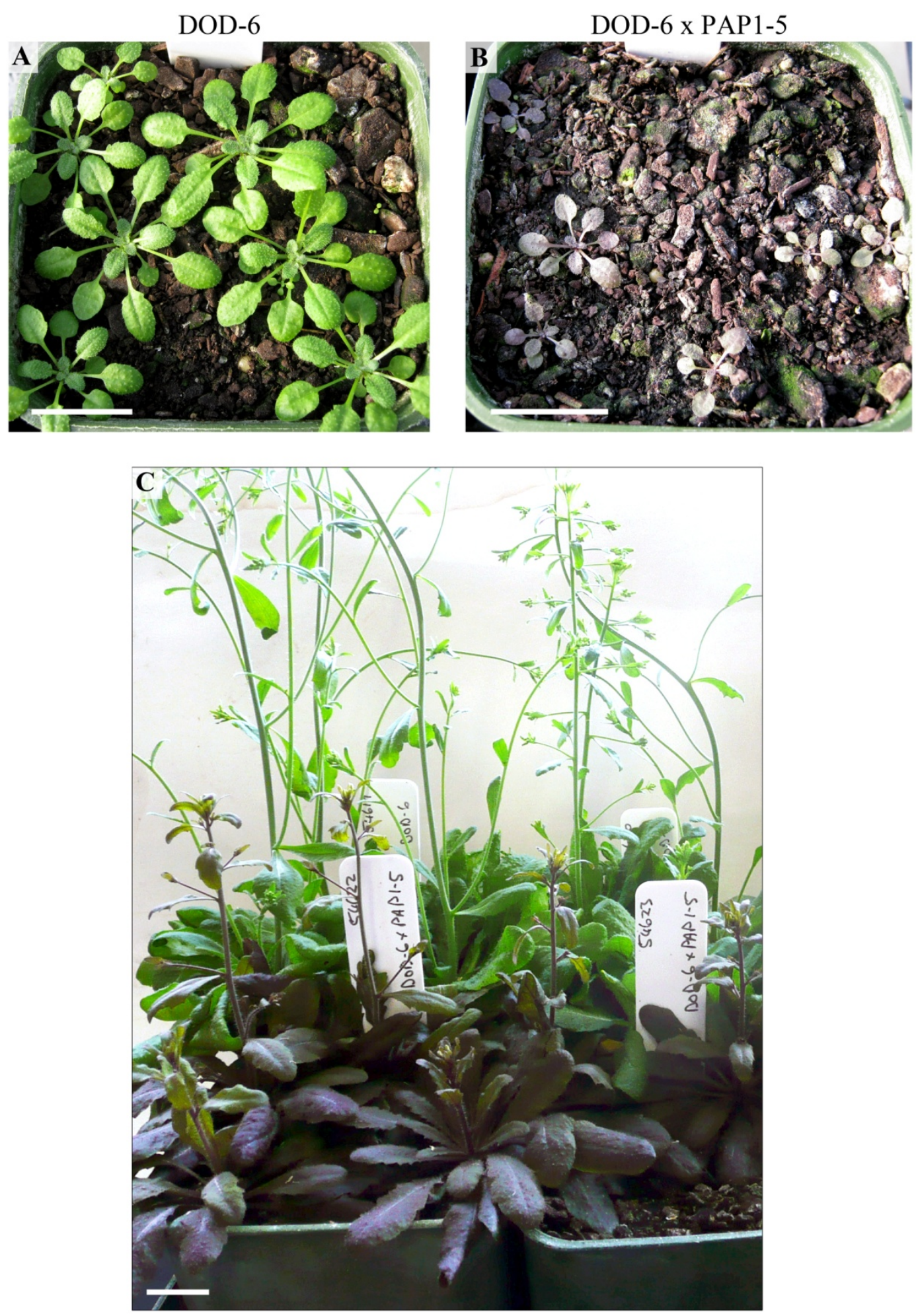

Figure 4.11 Slow germination and growth of DOD-6 x PAP1-5 plants compared with DOD-6. Comparisons made at (A and B) three weeks and (C) six weeks. In image $\mathbf{C}$, DOD-6 x PAP1-5 plants are in the front and DOD-6 at the back. Scale bars $=20 \mathrm{~mm}$. 

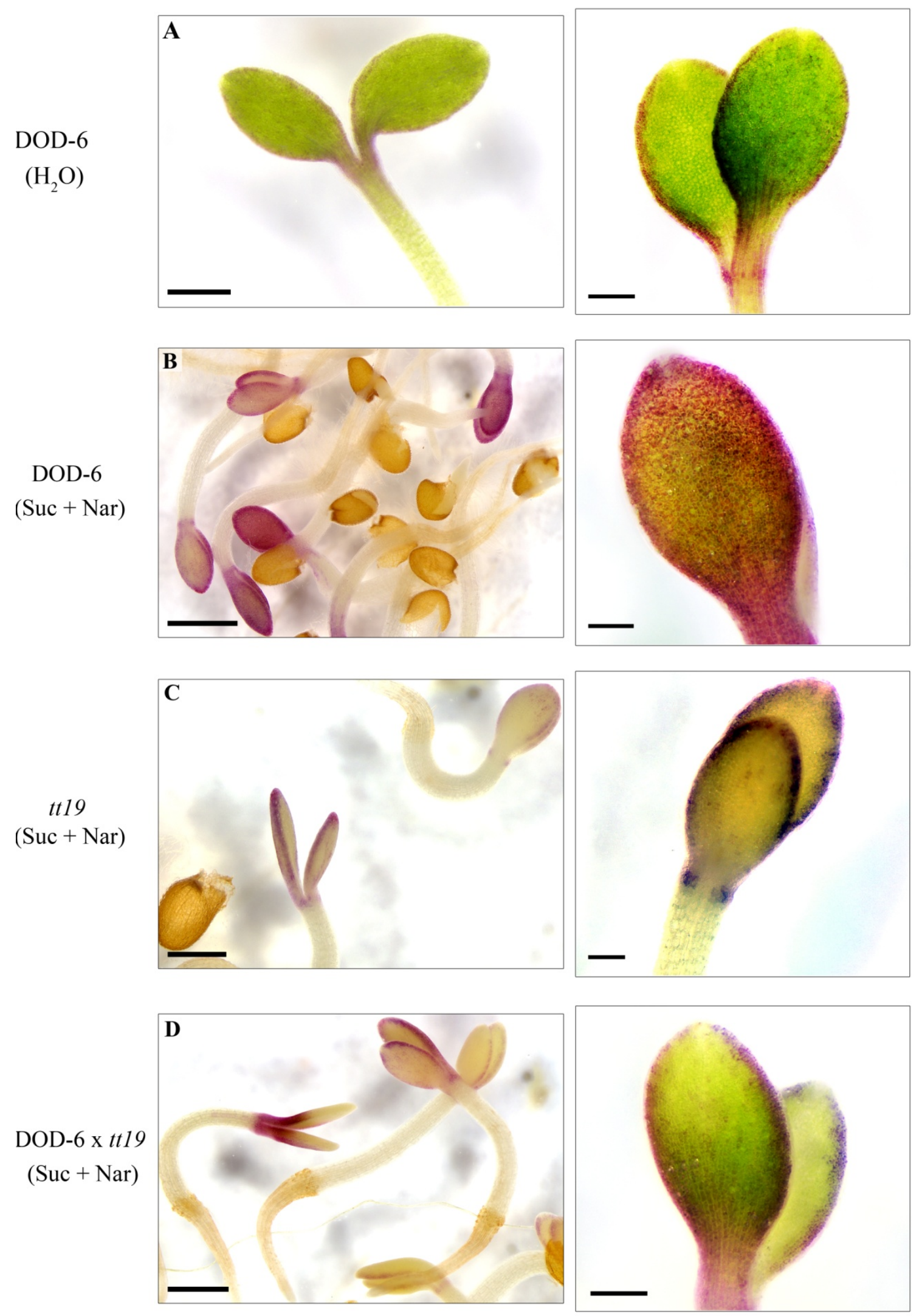

Figure 4.12 Anthocyanin induction in DOD-6, $t$ t19 and DOD-6 x $t$ t19 seedlings germinated in water or $3 \%(\mathrm{w} / \mathrm{v})$ sucrose liquid culture. Seedlings in sucrose were treated with $100 \mu \mathrm{M}$ naringenin. Scale bars: $\mathbf{A}, \mathbf{C}$ and $\mathbf{D}=250 \mu \mathrm{m}, \mathbf{B}=500 \mu \mathrm{m}$, close ups $=100 \mu \mathrm{m}$. 


\subsection{Discussion}

The focus of this chapter was to develop Arabidopsis as a model system to explore fundamental questions relating to anthocyanin and betalain pigment production. This system has the potential to be used for examining physiological differences between anthocyanic and betalainic plants. In addition, it could be used to study aspects of betalain biosynthesis, including transport and storage, and how they compare with anthocyanin biosynthesis. Both of these possibilities and their use of the model system are discussed.

\subsubsection{Physiological testing of betalains and anthocyanins}

Whether there are any physiological reasons behind the mutual exclusivity of betalain and anthocyanin pigments is unknown (Stafford, 1994). Betalains have been assumed to fulfil the same role as anthocyanins in plant tissue, yet this hypothesis has not been suitably tested (Stintzing \& Carle, 2004). The subcellular localisation of betalains described in Chapter 3 is comparable to published localisations of anthocyanins, which supports this replacement hypothesis. Using Arabidopsis, the assumption that betalains replaced anthocyanins could be tested by directly comparisons of the function of betalains and anthocyanins in the same tissues. Arabidopsis plants could be made that produce anthocyanins only (PAP1-5 plants), betalains only (DOD-6 plants), or both pigments (DOD-6 x PAP1-5 plants). These plants could then be exposed to a range of abiotic stressors such as cold, high light and salinity to measure differences in plant growth and physiology.

Development of a system to produce betalainic Arabidopsis plants for physiological testing, based on $35 S_{\text {pro }}$ :DOD transgenes, was problematic. DOD-6 plants required liquid feeding of L-DOPA to induce betalain production. However, L-DOPA quickly oxidised when in solution, making efficient uptake of this compound into plant tissues difficult. The addition of ascorbic acid to L-DOPA solutions reduced the oxidation of these compounds, but negatively affected plant growth (Section 4.3.2.3). If a less acidic antioxidant was found, hydroponic feeding of L-DOPA has the potential to produce healthy plants for physiological testing. Accumulation of betaxanthins was most efficient in growing tissues and slower in older tissue (Figure 4.5). Given these results, physiological testing of anthocyanins and betalains may need to be carried out at the 
seedling stage. The persistence of betalain pigments in these tissues after synthesis should also be further tested. However, as preliminary results with filter tip feeding suggest that the pigment levels were reduced with time, in spite of regular feeding of L-DOPA (Figure 4.8).

Two L-DOPA feeding methods were trialled for use in further analyses; filter paper and liquid feeding. Filter paper feeding of L-DOPA for transport analyses proved ineffective. L-DOPA oxidised quickly on filter paper resulting in slow pigment accumulation and the plants were susceptible to fungal infection (Section 4.3.2.1). Liquid culture feeding of L-DOPA was more successful. This method resulted in strong and consistent pigment accumulation (Figure 4.9), the speed of which could be modified depending on L-DOPA concentration. If analyses required only short term production of betaxanthin, such as to examine pigment transport, the health of the plants and longevity of pigments in the tissues would not be a problem. Another benefit for this feeding method is that seedlings are surrounded by solution, which is ideal for feeding additional chemicals to the plants such as naringenin (used in anthocyanin accumulation Section 4.3.5.2) or various transport inhibitors.

In addition to L-DOPA feeding issues, a drawback of physiological testing using DOD-6 Arabidopsis is that betaxanthins may not be directly comparable to anthocyanins as they have different spectral qualities. Production of betacyanins in these plants would be important for this purpose as they are more similar to anthocyanins. The ability of DOD-6 plants to produce both betacyanins and betaxanthins was tested by feeding cyclo-DOPA along with L-DOPA. From the filter paper feeding trials, it was found that the production of betacyanins is possible in DOD-6 tissues, but even more problematic than producing betaxanthins alone. Addition of cyclo-DOPA to the L-DOPA solution to induce betacyanin production resulted in fast oxidation of cyclo-DOPA to a red/black compound (Figure 4.6A), which may be dopachrome. The compound cyclo-DOPA is known to be unstable in solution, where it can rapidly oxidise to form the pigment dopachrome (Young et al., 1974). The addition of ascorbic acid was only slightly effective at reducing oxidation (Section 4.3.2.1). Also, plants fed with L-DOPA + cyclo-DOPA and ascorbic acid through hydroponic methods, appeared unhealthy (Figure 4.7), which was likely due to acidification of the solution. Attempts 
to buffer the $\mathrm{pH}$ of this solution to prevent this was unsuccessful, as the feeding method promoted oxidation of cyclo-DOPA to dopachrome (Section 4.3.2.2).

To avoid the need to feed cyclo-DOPA, a cDNA for the betalain cytochrome P450 gene, CYP76AD1, was isolated from Swiss chard and transformed into DOD-6. If successful, DOD-6/CYP76AD1 plants would not need to be fed cyclo-DOPA, requiring only L-DOPA to produce both classes of betalains. Nakatsuka et al. (2013) recently made a self-contained betalain biosynthetic pathway in Arabidopsis cell cultures. These cultures were transformed with a mushroom tyrosinase, the enzyme responsible for conversion of tyrosine to L-DOPA in the fungal melanin biosynthetic pathway. DOD from the four o'clock plant (Mirabilis jalapa) was also transformed into these cultures. As a result Arabidopsis cell cultures made betaxanthins without feeding L-DOPA. In the future, a similar tyrosinase could be inserted into DOD-6/CYP76AD1 plants for a self-contained betalain pigment system which requires only three enzymatic steps without the need to feed L-DOPA. One caveat to such a system in Arabidopsis, is that there could be issues with the constitutive expression of a tyrosinase. In the study by Nakatsuka et al. (2013), the cell cultures containing tyrosinase turned brown with time. However, if the Arabidopsis plants with a tyrosinase, $D O D$ and $C Y P 76 A D 1$ were successful at producing betalains, then this model system would have the potential for physiological testing without physiological stress caused by plants being fed solutions.

Another benefit of the Arabidopsis model for physiological testing of betalains is the availability of known stress sensitive Arabidopsis mutants. In addition to a direct physiological comparison between plants containing PAP1-5 and DOD-6, these Arabidopsis plants could also be crossed with available stress sensitive Arabidopsis mutants. Mutants such as Salt Overly Sensitive 1 (SOS1; Shi et al. (2000)) and the frost sensitive mutant Inducer of CBF Expression 1 (ICE1; Chinnusamy et al. (2003)) are readily available through ABRC. These mutants would allow testing for improved physiological performance under abiotic stress in plants that produce betalains, anthocyanins, or both. One issue that could be of future consequence for these physiological experiments, however, is the difference in growth rate for plants that contain the PAP1 transgene. It was observed that both PAP1-5 and DOD-6 x PAP1-5 plants germinated later, produced fewer leaves and were slower to flower than DOD-6 plants (Figure 4.11). Due to differences in growth morphology, this would have to be 
taken into account for any physiological comparison against DOD-6 or Columbia plants. It may be useful to develop a transgenic line that confers anthocyanin production at lower levels or, for example, only in the leaves.

\subsubsection{Conclusions and future directions}

Aspects of betalain biosynthesis could be studied using the Arabidopsis pigment model system. DOD-6 and the crosses, DOD-6 x PAP1-5, DOD-6 x $t$ t12, and DOD-6 x $t$ t 19 could be used for analyses on mechanisms of betalain transport from site of synthesis on the endoplasmic reticulum to storage in the vacuole. The current genotype information known about each line and the analyses still needing to be done are outlined below.

- DOD-6 x PAP1-5: both $D O D$ and PAP1 inserts require selection before use. It is unknown if these plants are homozygous or heterozygous for the insertions. Although not required for further analyses in this thesis, full characterising of the genotype for DOD-6 x PAP1-5 is possible. One method that may be used is sending plants to be sequenced. $\mathrm{F}_{4}$ seed is available for this cross.

- DOD-6 x $t t 12$ : this cross is genotyped as homozygous for the $t t$ T-DNA insert, but whether $D O D$ is homozygous or heterozygous is unknown. The presence of $D O D$ would need to be screened for individual plants before use. As for the cross DOD-6 x PAP1-5, full characterisation of the genotype could be achieved through sequencing. $F_{3}$ seed is available for this cross.

- DOD-6 x tt19: this line was phenotyped as homozygous for the $t t$ T-DNA insert. Whether $D O D$ is homozygous or heterozygous is unknown. The presence of $D O D$ would need to be screened for individual plants before use. Further characterisation is not necessary for this thesis. However, for future experiments it would be beneficial to fully characterise this cross. As for the other two $D O D$ crosses, these plants could be sent for sequencing. $F_{3}$ seed is available for this cross. 


\section{Chapter 5: Storage and transport}

\subsection{Introduction}

It is unknown how betalain pigments are transported from site of synthesis to storage in the vacuole. Betalain biosynthetic enzymes are thought to be located on the cytoplasmic side of the endoplasmic reticulum (ER) (Gandía-Herrero \& García-Carmona, 2012), similar to anthocyanins (Hrazdina et al., 1978). As demonstrated in Chapter 3, betalains and anthocyanins have similar pigmentation patterns in vegetative tissues. Therefore, similar biosynthetic control mechanisms, including transport systems, seem likely. The cellular transport of anthocyanin and proanthocyanidin molecules, as discussed in Section 1.6, is a complicated topic with conflicting evidence from different model systems. Unlike flavonoid biosynthesis which is well characterised, flavonoid vacuolar transport has no unified model. A number of transport mechanisms have been described (Zhao \& Dixon, 2010), but it is unknown how they all fit together. Information acquired to date was facilitated by the availability of mutants, and the use of transport inhibitors that result in visible phenotypes when transport is affected (Grotewold, 2004). The main difficulty in investigating flavonoid transport, however, seems to be from the large number of different components involved.

It is now known that both anthocyanins and proanthocyanidins use a direct transport route from synthesis on the ER, to storage in the vacuole (Poustka et al., 2007). Rather than being processed through the trans-Golgi network (TGN), these compounds use either vesicle-like structures for mass transport (Gomez et al., 2011; Zhang et al., 2006), or a cytoplasmic route. Transport through the cytoplasm is thought to be aided by glutathione $S$-transferase-like (GST-like) carrier proteins (Zhao \& Dixon, 2010) and a couple of different membranous transport proteins. One of these transport proteins, a multidrug and toxic extrusion (MATE) transporter, appears proanthocyanidin-specific (Marinova et al., 2007). Exact localisation of this MATE transporter is unknown, and may be on vesicles, tonoplast membranes, or both (Debeaujon et al., 2001). The other type of transporter identified in flavonoid transport is an ATP-binding cassette (ABC) (Francisco et al., 2013; Goodman et al., 2004; Lu et al., 1997). ABCs are structurally distinct from MATE transporters (Debeaujon et al., 2001) and were demonstrated to be 
involved with GST-like proteins (Francisco et al., 2013), suggesting that ABC transporters involved may be glutathione (GS-X) pumps.

To investigate the possible involvement of these transporters in betalain accumulation, an artificial Arabidopsis thaliana betalain model system, based on DOD-6, was developed in Chapter 4. Transport analyses using this model system are ideal. With the genome of Arabidopsis freely accessible and a wide variety of pigmentation mutants, it is quite a malleable system. Also, the autofluorescence of betaxanthins allows accumulation these compounds to be monitored without the use of fluorescent markers. Different known flavonoid transporters were tested using DOD-6, Arabidopsis mutants, and transport inhibitors as described below and illustrated in Figure 5.1.

- Betaxanthin transport via the Golgi network (Figure 5.1A) was tested using brefeldin A (BFA). BFA is an inhibitor which acts to break down the structure of the Golgi network. This inhibitor had no effect on anthocyanin transport in Arabidopsis (Poustka et al., 2007), which provided evidence for a direct ER-to-vacuole transport route.

- Vesicular transport of betaxanthins was investigated (Figure 5.1B). Subcellular accumulation of betaxanthins was examined in DOD-6. In addition, the involvement of autophagy in the creation of vesicles was tested using 3-methyladenine (3-MA), an autophagy inhibitor. This inhibitor had no effect on anthocyanin accumulation in Arabidopsis (Poustka et al., 2007).

- Transport via a MATE transporter (Figure 5.1C) was tested using the Arabidopsis MATE transporter mutant $t t 12$, in the DOD- 6 x $t t 12$ cross. In addition, DOD-6 was treated with the chemical MATE inhibitor, pyrimethamine (PYR).

- Betaxanthin transport by GST-like proteins (Figure 5.1D) was tested using the GST Arabidopsis mutant $t t 19$ in the cross DOD-6 x $t t 19$.

- Involvement of $\mathrm{ABC}$ transporters (Figure 5.1E) was tested by the inhibition of ABCs with vanadate (VAN), a general ATPase inhibitor which includes ABCs. 


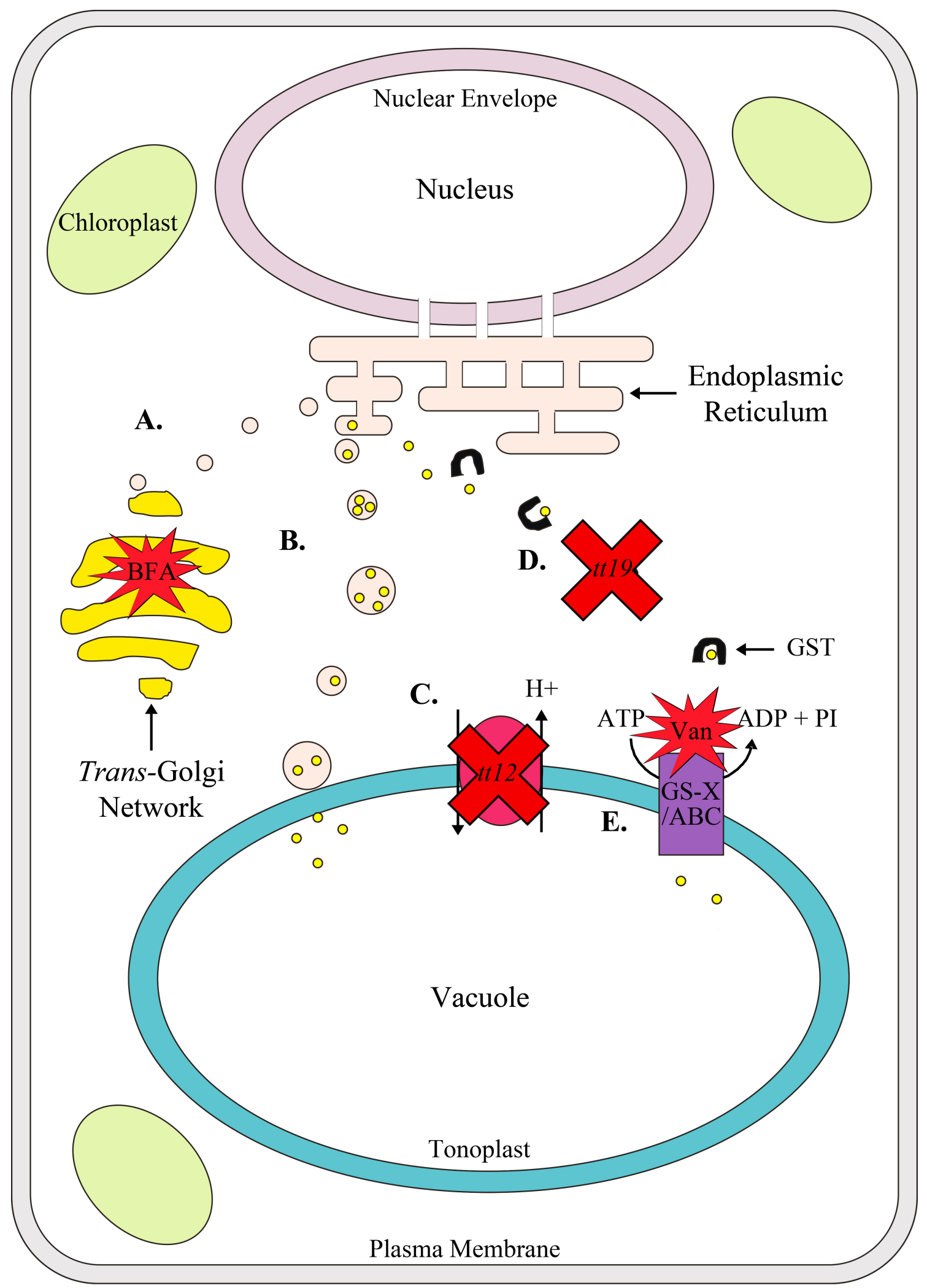

Figure 5.1 Illustration of betaxanthin transport hypotheses tested in this chapter. (A) Trans-Golgi network-mediated transport route, treated with Brefeldin A (BFA); (B) Vesicular transport; (C) Multidrug and toxic extrusion (MATE) transporter knockout mutant, $t t 12$; (D) Glutathione $S$-transferase (GST) carrier protein knockout mutant, $t t 19$; (E) Glutathione/ ATP-binding cassette (GS-X/ABC) transporter, inhibited by vanadate (Van). 


\subsubsection{Aim}

The aim of this chapter was to compare the vacuolar storage and transport mechanisms of betalains and flavonoids. As well as examine the potential for anthocyanin and betalain pigments to co-localise using DOD-6 x PAP1-5.

\subsection{Methods}

\subsubsection{Phenotyping of Arabidopsis lines}

Phenotypic observations of betalain and anthocyanin pigment distribution were made in the following Arabidopsis lines: wild type Columbia, DOD-6, PAP1-5, DOD-6 x PAP1-5, DOD-6 x $t$ t12 and DOD-6 x $t$ t19. Given that all crosses were pooled seed rather than individual lines, multiple plants were assessed for each cross. Feeding of $10 \mathrm{mM}$ L-DOPA to whole plants was carried out on filter paper (Section 4.2.2.1) and with liquid culture (Section 4.2.2.4). Changes in pigmentation were observed with microscopy (Section 2.4).

\subsubsection{Anthocyanin and betalain induction in DOD-6}

Anthocyanin pigmentation was induced in Columbia and DOD-6 Arabidopsis, similar to Section 4.2.5.4, with the following changes. After three days in the rotary shaker, the germinated seedlings in both the sucrose and water solutions the following treatments were added: water (negative control), $100 \mu \mathrm{M}$ naringinen (100 mM stock in EtOH), 10 mM L-DOPA (Appendix 1.7.1), 10 mM L-DOPA + 1 mM naringenin.

\subsubsection{Transport inhibition assays}

Transport inhibition assays were adapted from methods by Poustka et al. (2007).

DOD-6 and DOD-6 x PAP1-5 seeds were surface sterilised and germinated in liquid culture as in Section 4.2.2.4. After three days in the rotary shaker, the seedlings were fed with either water or $1 \mathrm{mM}$ L-DOPA. The following transport inhibitors were used: $2.5 \mathrm{mM}$ pyrimethamine (PYR), $10 \mathrm{mM}$ brefeldin A (BFA), $1 \mathrm{mM}$ vanadate (VAN) and $30 \mathrm{mM}$ 3-methyladenine (3-MA). Inhibitors were added to the liquid culture $3 \mathrm{~h}$ before feeding L-DOPA, except for 3-MA, which was added $12 \mathrm{~h}$ before feeding L-DOPA. 


\subsubsection{Anthocyanin and betalain extractions}

Betalains were extracted from L-DOPA fed DOD-6 and red Swiss chard leaves, and anthocyanins were extracted from DOD-6 x PAP1-5 leaves. The extracts were carried out on freshly ground whole seedlings using acidified $\mathrm{MeOH}(99: 1 \mathrm{MeOH}: \mathrm{HCl})$ for $3 \mathrm{~h}$ at $4{ }^{\circ} \mathrm{C}$. Extractions were then centrifuged and supernatant transferred into new tubes.

\subsection{Results}

\subsubsection{Betalains and anthocyanins can co-localise}

\subsubsection{Comparison of DOD-6, PAP1-5 and DOD-6 x PAP1-5}

DOD-6 plants fed with L-DOPA produced betaxanthins in most, if not all, cell types (Figure 5.2). Green autofluorescence, used to detect the presence of betaxanthins, was strongly visible in these sections under blue light. In DOD-6 x PAP1-5, anthocyanins were mainly localised to cells surrounding vascular tissue and in epidermal tissue (Figure 5.3). When DOD-6 x PAP1-5 was fed with L-DOPA, betaxanthins appeared to accumulate in cells that did not contain anthocyanins (Figure 5.4A and 5.4B). The hypocotyl epidermal cells lacked green autofluorescence, suggesting that there was no betaxanthins in these cells. However, L-DOPA fed DOD-6 x PAP1-5 lamina sections examined using the confocal microscope, revealed that most purple cells had low levels of green autofluorescence. This indicated that betaxanthins and anthocyanins were co-localising within the same vacuole. That these cells had weaker autofluorescence than a neighbouring cell without anthocyanins (Figure 5.4C), suggested reductions in autofluorescence were either due to pigment storage limitations in the vacuole, or fluorescence quenching of betalains by anthocyanins. Possibilities were examined by experiments that induced high levels of anthocyanin and betalain accumulation at the same time in DOD-6 (Figure 5.5). Sucrose + naringenin enhanced anthocyanin accumulation in DOD-6 more than sucrose alone (Figure 5.5B and C). Based on pigment colour intensity, L-DOPA fed DOD-6 (Figure 5.5D) produced the same amount or even slightly less betaxanthins than the sucrose + L-DOPA (Figure 5.5E) or sucrose + naringenin + L-DOPA treatments (Figure 5.5F). Under blue light, DOD-6 seedlings with both betalains and anthocyanins did indeed have less autofluorescence in the areas where anthocyanin accumulation was the greatest. Whether the reduced autofluorescence was largely due to betaxanthin autofluorescence quenching, was 
examined by extracting betalain and anthocyanin pigments, then combining to view autofluorescence. Mixing of betaxanthin extractions with betacyanin or anthocyanin extracts reduced betaxanthin autofluorescence, although, betaxanthin autofluorescence still remained visible (Figure 5.6). Betaxanthin fluorescence quenching explains the confocal observations, where anthocyanic cells in the lamina had reduced autofluorescence (Figure 5.4C). However, the cause for hypocotyl epidermal cells without betaxanthin autofluorescence in Figure 5.4B, remains unexplained.

\subsubsection{Transport of betalains}

\subsubsection{Betaxanthins appear to have vesicular transport}

Betaxanthins accumulating in DOD-6 hypocotyl cells fed with L-DOPA was clearly seen using confocal microscopy (Figure 5.7). All green autofluorescence was from betaxanthins, apart from green autofluorescence that co-localised with red autofluorescence, which was probably carotenoid autofluorescence within chloroplasts (as found in Chapter 3). The solid green autofluorescence observed in a number of cells was likely due to betaxanthins filling the vacuoles. In neighbouring cells, betalains were undergoing vacuolar accumulation (Figure 5.8). There were strings of autofluorescence across some cells which appeared similar to cytoplasmic movement as described by Verchot-Lubicz and Goldstein (2010). In cells with less autofluorescence in the vacuole, circular structures of various sizes contained betaxanthin compounds. Most of these structures were below $1 \mu \mathrm{m}$ or $1-4 \mu \mathrm{m}$ in diameter, with the largest diameter of a membrane-bound compartment $15 \mu \mathrm{m}$. These structures appeared similar to vesicles and pre-vacuolar compartments (PVCs) observed in anthocyanin-producing species (Gomez et al., 2011; Poustka et al., 2007; Zhang et al., 2006).

\subsubsection{2 $t t$ mutants affect betaxanthin transport}

Betaxanthin autofluorescence, as visibly assessed, was reduced in L-DOPA fed DOD-6 $\mathrm{x} t t 12$ (Figure 5.9) and DOD-6 x $t t 19$ flower stalks (Figure 5.10) when compared with DOD-6 (Figure 5.2). Petal pigmentation was also reduced in comparison with DOD-6 under white light. Also, sepals on DOD-6 x tt19 flowers had dotted autofluorescence, rather than the consistent glow seen on DOD-6 sepals. Cross sections of DOD-6 x tt12 (Figure 5.11) and DOD-6 x $t$ t19 (Figure 5.12) flower stalks appeared similar to unpigmented Columbia under white light (Figure 5.2). Under blue light, the only green 
autofluorescence seen in these crosses was in the guard cells (Figures 5.11 and 5.12). Autofluorescent guard cells could be why the stalks were weakly fluorescent in the whole flower micrographs (Figures 5.9 and 5.10). Confocal analyses of L-DOPA fed DOD-6 x tt19 seedlings, saw differences in pigment accumulation compared with DOD-6 (Figure 5.13). Under confocal, autofluorescence in these cells was blotchy in the cytoplasm, unlike DOD-6 tissue which had more consistent autofluorescence in similar cells. In some cells, it was clear that vacuoles contained no autofluorescence (Figure 5.14). This indicated that vacuolar accumulation of betaxanthin may have been abolished in DOD-6 x tt19.

\subsubsection{Chemical inhibition of betaxanthin transport}

VAN strongly affected betaxanthin accumulation in DOD-6 seedlings (Figure 5.15). Seedlings did not visibly produce much yellow pigment under white light, but still had weak green autofluorescence. On close inspection of lamina tissue, cells did not appear to be consistently autofluorescent. The hypocotyl tissue of these seedlings was then sectioned and analysed with confocal (Figure 5.16). L-DOPA fed DOD-6 cells had pigment accumulated in the vacuoles (Figure 5.16A), whereas, those treated with VAN appeared to have most of the betaxanthins accumulate in the cytoplasm (Figure 5.16B). Other transport inhibitors tested, PYR, BFA and 3-MA, had no visible affect on betaxanthin accumulation and autofluorescence (Figure 5.17). However, when DOD-6 x PAP1-5 (Figure 5.18A) was treated with PYR, the production of structures resembling anthocyanic vacuolar inclusions (AVIs, see Section 1.6) (Zhang et al., 2006) was induced (Figure 5.18B). Feeding with L-DOPA also resulted in the formation of AVIs (Figure 5.18C), but were not as visually abundant as when fed with PYR. Formation of AVI-like structures was not seen in DOD-6 plants fed with L-DOPA + naringenin (Figure 5.5), only in DOD-6 x PAP1-5 seedlings. The treatment of PYR + L-DOPA resulted in larger and less regular looking AVIs (Figure 5.18D). Epidermal sections of DOD-6 x PAP1-5 lamina treated with PYR and PYR + L-DOPA revealed the difference in AVI appearance. AVIs produced by PYR treated seedlings were around $3-7 \mu \mathrm{m}$ in diameter and dense in colour (Figure 5.19A). In contrast, most of the AVI structures in L-DOPA + PYR fed DOD-6 x PAP1-5 appeared to contain betaxanthins as well as anthocyanins. These structures appeared to autofluoresce green under blue light, and had a larger diameter of 9 - $13 \mu \mathrm{m}$ (Figure 5.19B). 

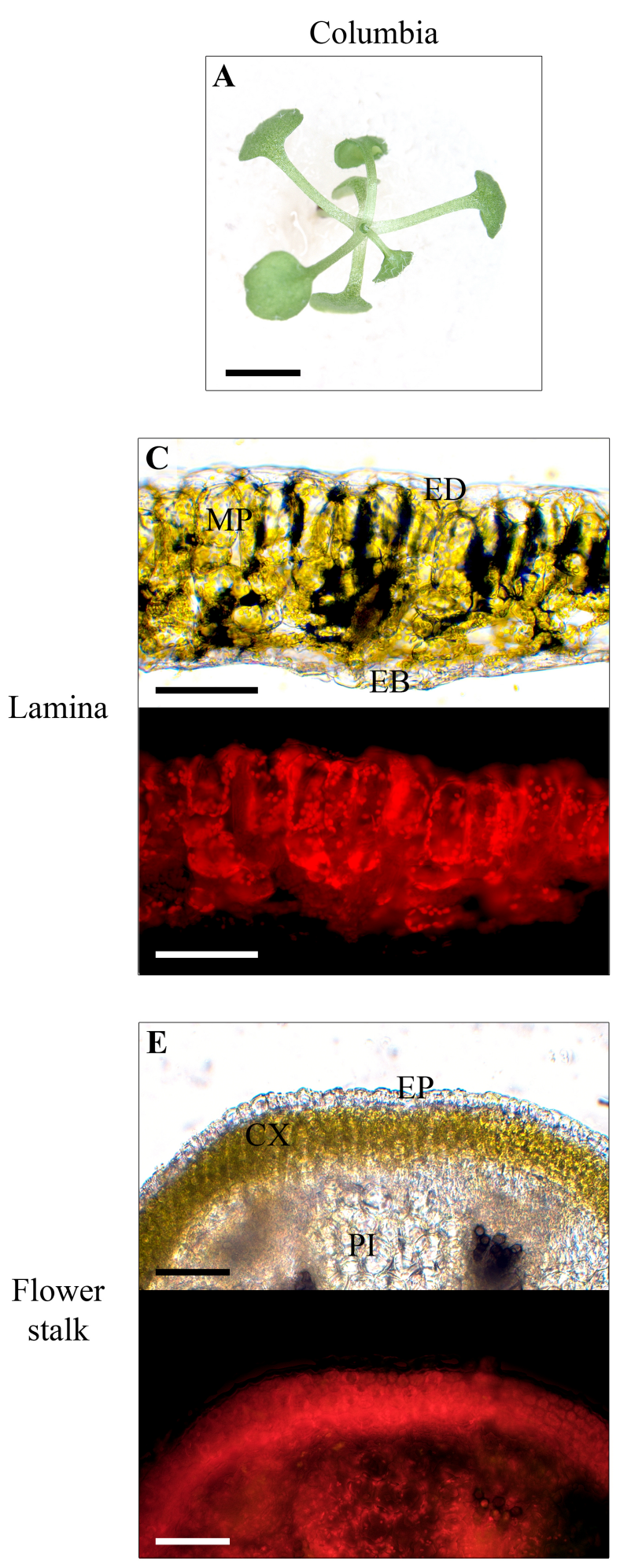
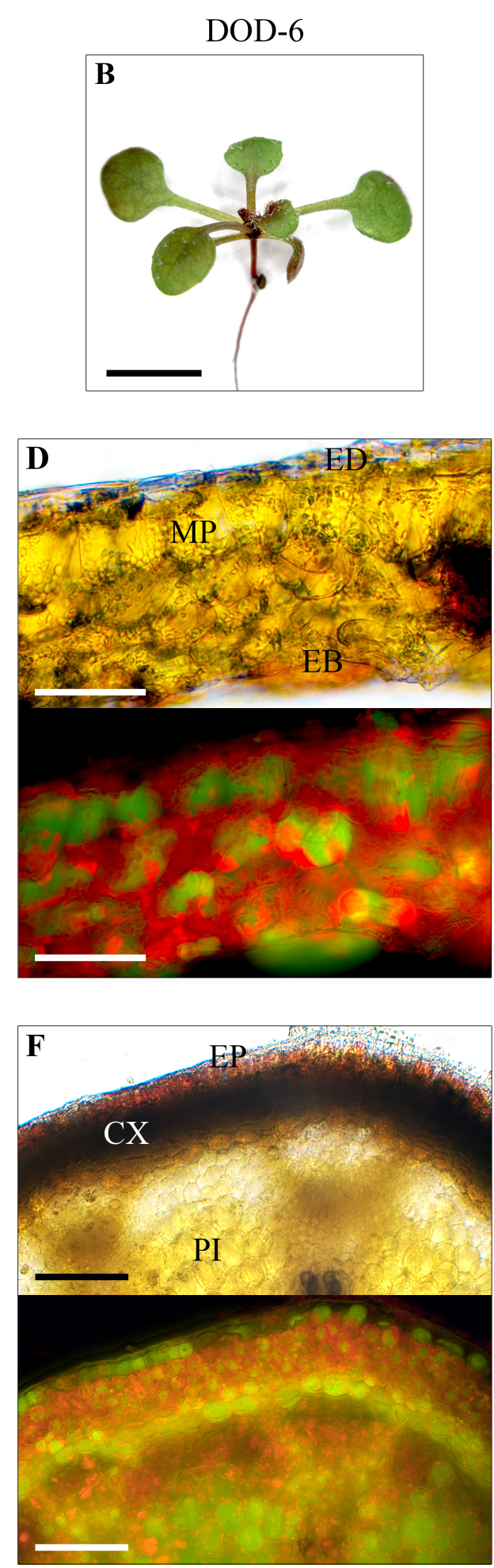

Figure 5.2 Betaxanthin localisation in (A) Columbia and (B) DOD-6 plants following L-DOPA feeding. Transverse sections of (C) Columbia and (D) L-DOPA fed DOD-6 lamina, (E) Columbia flower stalk and (F) L-DOPA fed DOD-6 flower stalk, under white and blue light. Key: CX cortex, EB abaxial epidermis, ED adaxial epidermis, EP epidermis, MP palisade mesophyll, PI pith. Scale bars: $\mathbf{A}$ and $\mathbf{B}=5 \mathrm{~mm}, \mathbf{C}$ and $\mathbf{D}=$ $200 \mu \mathrm{m}, \mathbf{E}$ and $\mathbf{F}=100 \mu \mathrm{m}$. All sections are unstained. 
Columbia

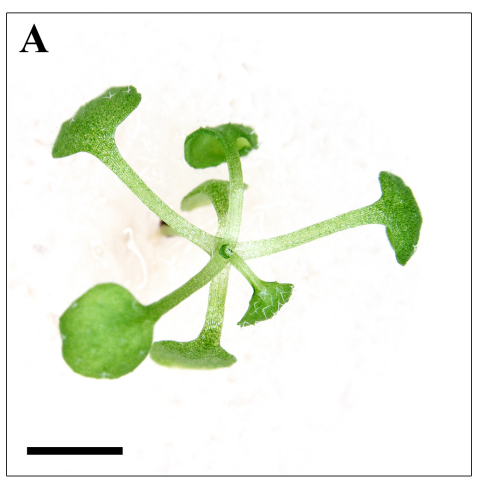

DOD-6 x PAP1-5
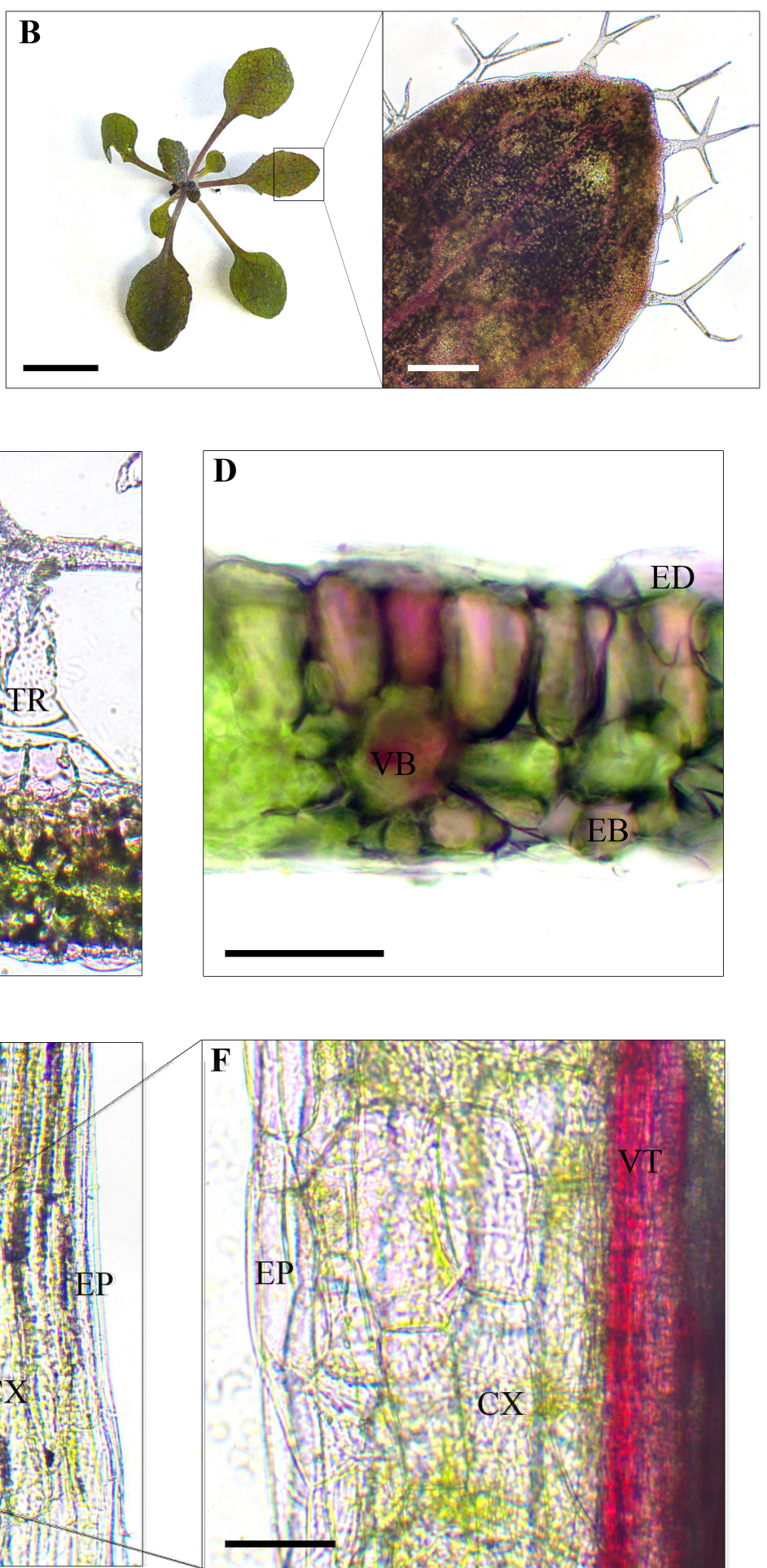

Figure 5.3 Anthocyanin localisation in DOD-6 x PAP1-5 sections. (A) Columbia and (B) DOD-6 x PAP1-5 seedlings with insert of leaf close-up. DOD-6 x PAP1-5 (C and D) lamina transverse sections and ( $\mathbf{E}$ and $\mathbf{F}$ ) hypocotyl longitudinal sections. Key: CX cortex, EB abaxial epidermis, ED adaxial epidermis, EP epidermis, TR trichome, VB vascular bundle, VT vascular tissue. Scale bars: $\mathbf{A}$ and $\mathbf{B}=5 \mathrm{~mm}(\mathbf{B}$ insert $=450 \mu \mathrm{m})$, $\mathbf{C}$ and $\mathbf{D}=200 \mu \mathrm{m}, \mathbf{E}=100 \mu \mathrm{m}, \mathbf{F}=50 \mu \mathrm{m}$. All sections are unstained. 

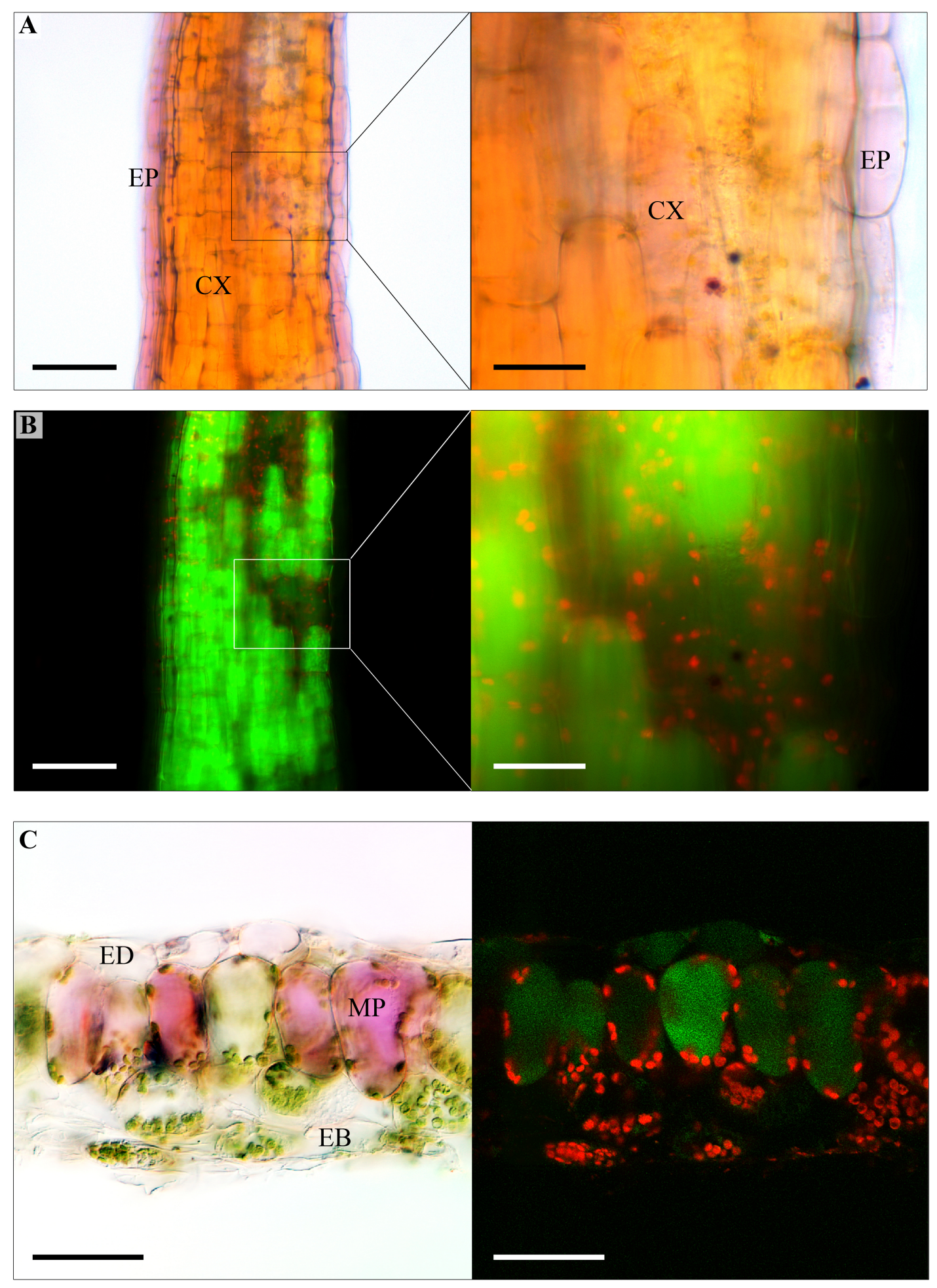

Figure 5.4 Anthocyanin and betalain co-localisation in L-DOPA fed DOD-6 x PAP1-5 sections. A hypocotyl longitudinal section under (A) white light and (B) blue light using compound microscopy. Green autofluorescence betaxanthins, red autofluorescence chloroplasts. (C) Confocal images of a lamina transverse section with (left) overlaying colour channels blue, yellow and red, and (right) autofluorescence at $488 \mathrm{~nm}$ excitation and $498-560 \mathrm{~nm}$ emission for betaxanthins (green) and $625-732 \mathrm{~nm}$ emission for chloroplasts (red). Key: CX cortex, EB abaxial epidermis, ED adaxial epidermis, EP epidermis, MP palisade mesophyll. Scale bars: $\mathbf{A}, \mathbf{B}$ and $\mathbf{C}=150 \mu \mathrm{m}$, inserts $=50 \mu \mathrm{m}$. All sections are unstained. 


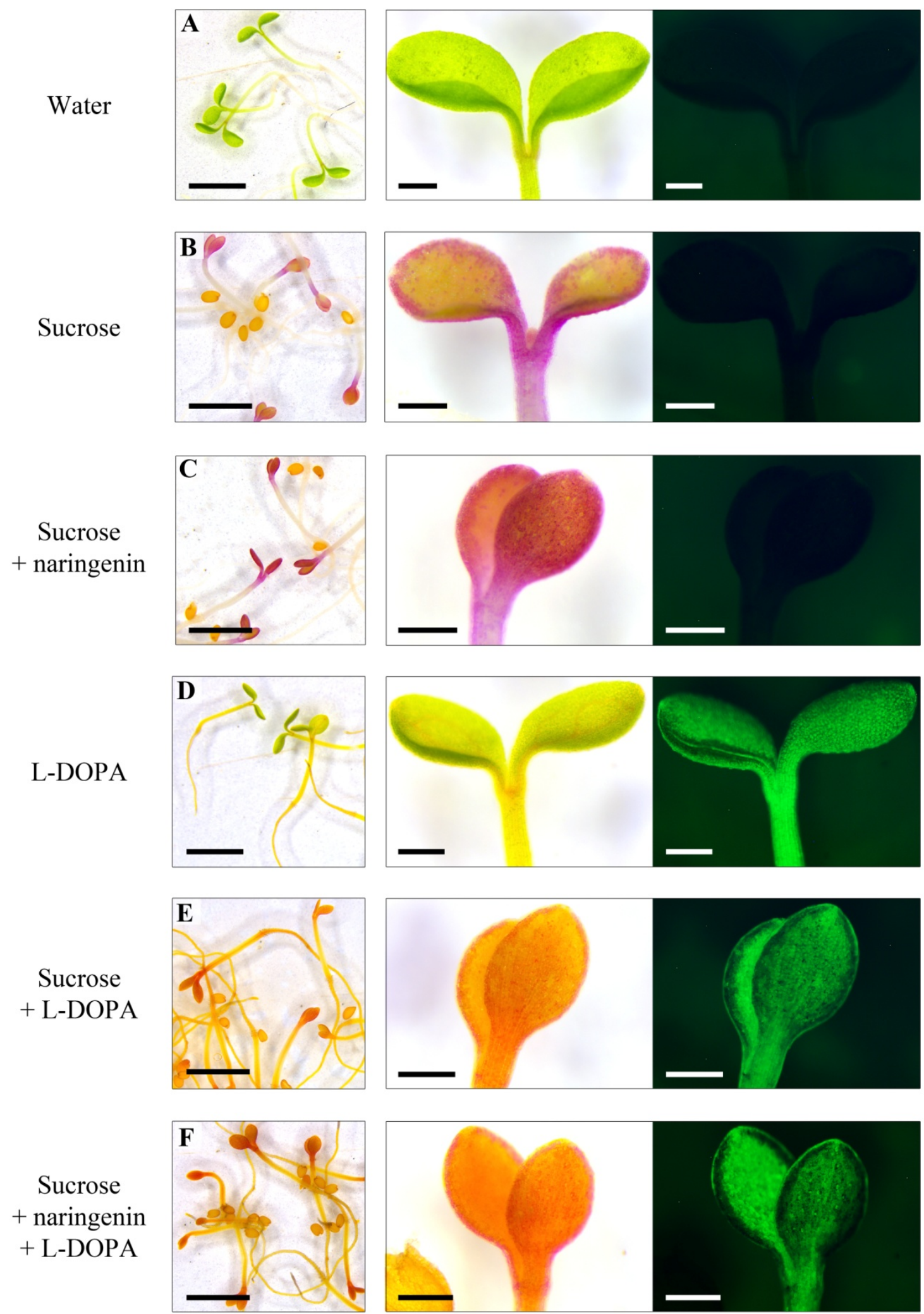

Figure 5.5 Co-localisation of anthocyanins and betalains in DOD-6 seedlings by anthocyanin induction and L-DOPA feeding. White light and blue light images of (A) water control, (B) sucrose, (C) sucrose + naringenin, (D) L-DOPA, (E) sucrose + L-DOPA, $(\mathbf{F})$ sucrose + naringenin + L-DOPA. Scale bars: $\mathbf{A}-\mathbf{F}=2 \mathrm{~mm}$, close up images $=250 \mu \mathrm{m}$. 

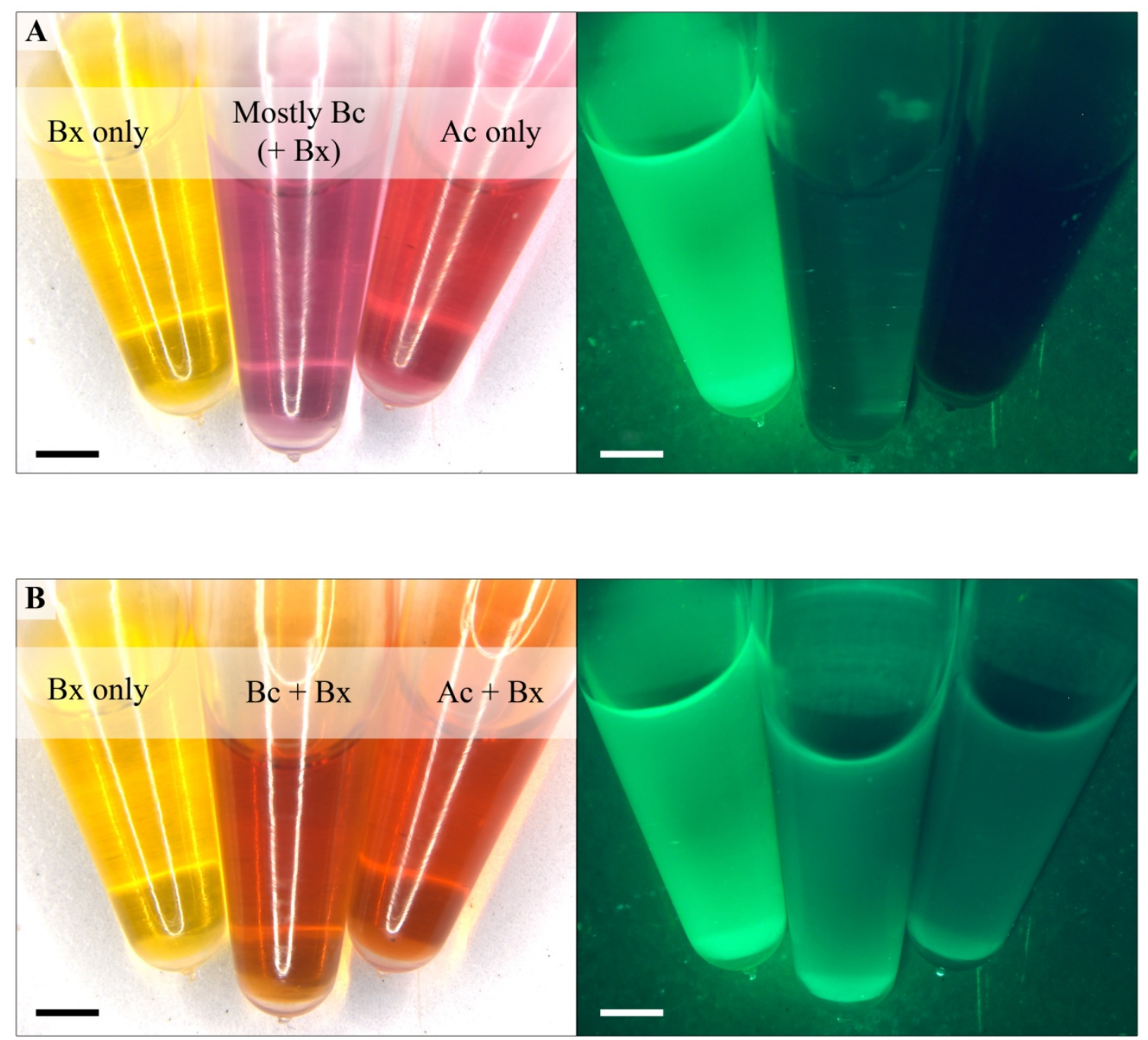

Figure 5.6 Extractions of anthocyanins and betalains to demonstrate betaxanthin autofluorescence quenching in the presence of betacyanins and anthocyanins. Dissecting microscopy white and blue light images of (A) acidified methanol extractions of LDOPA fed DOD-6 (Bx only, left), purple Swiss chard (Mostly Bc (+ Bx), middle) and DOD-6 x PAP1-5 (Ac only, right) tissues, and (B) 50:50 mixture of extractions in image $\mathbf{A}$ with either acidified methanol (left) or Bx only extraction (middle and right). Key: Ac anthocyanins, Bc betacyanins, Bx betaxanthins. Scale bars $=2 \mathrm{~mm}$. 

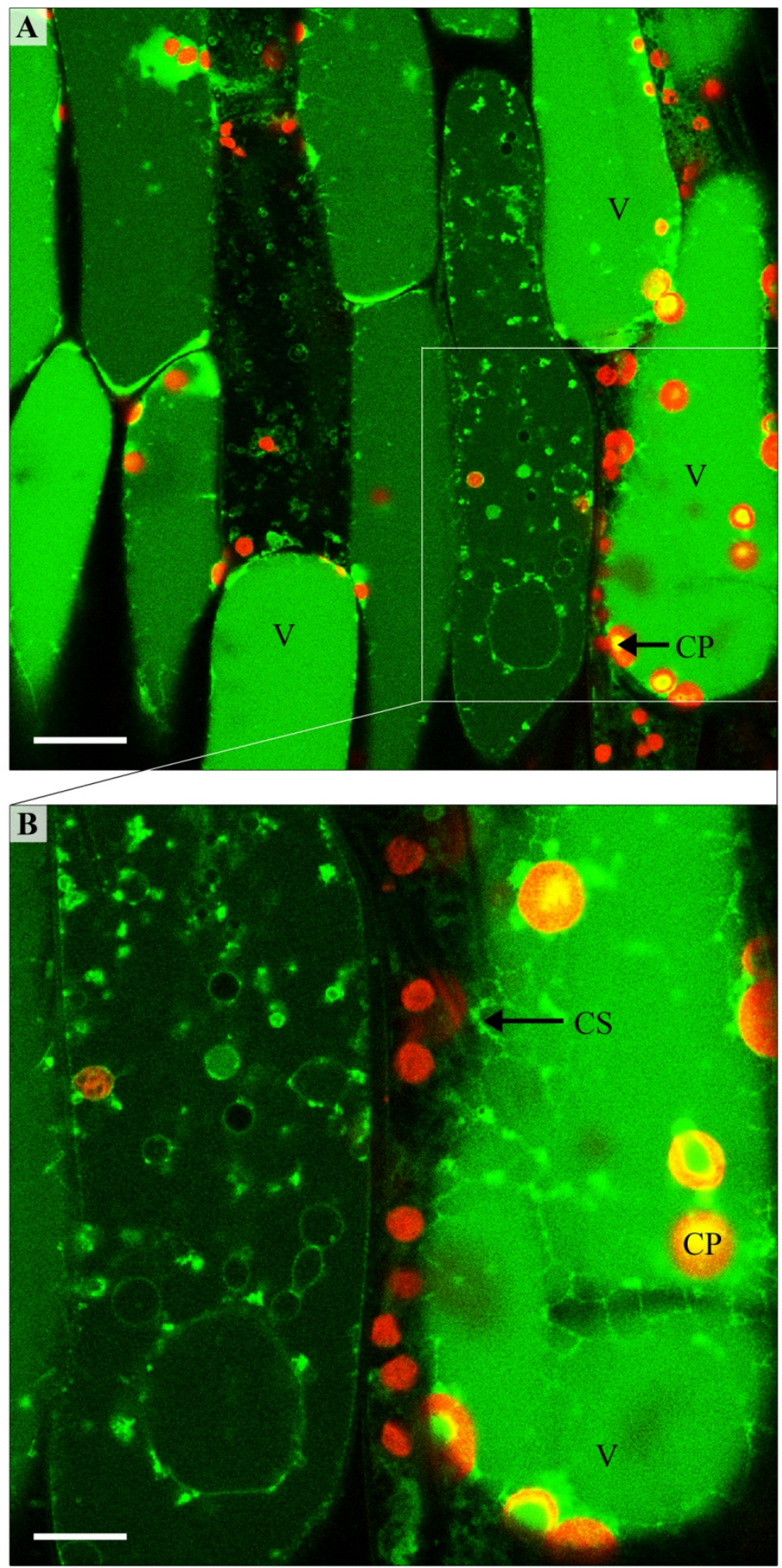

Figure 5.7 Confocal analyses of betaxanthin accumulation in L-DOPA fed DOD-6 hypocotyl tissue. Autofluorescence at $488 \mathrm{~nm}$ excitation with 498 - $560 \mathrm{~nm}$ emission spectrum for betaxanthins (green) and $625-732 \mathrm{~nm}$ emission spectrum for chloroplasts (red). Key: CP chloroplast, CS cytoplasmic movement, V vacuole. Scale bars: $\mathbf{A}=$ $20 \mu \mathrm{m}, \mathbf{B}=10 \mu \mathrm{m}$. 


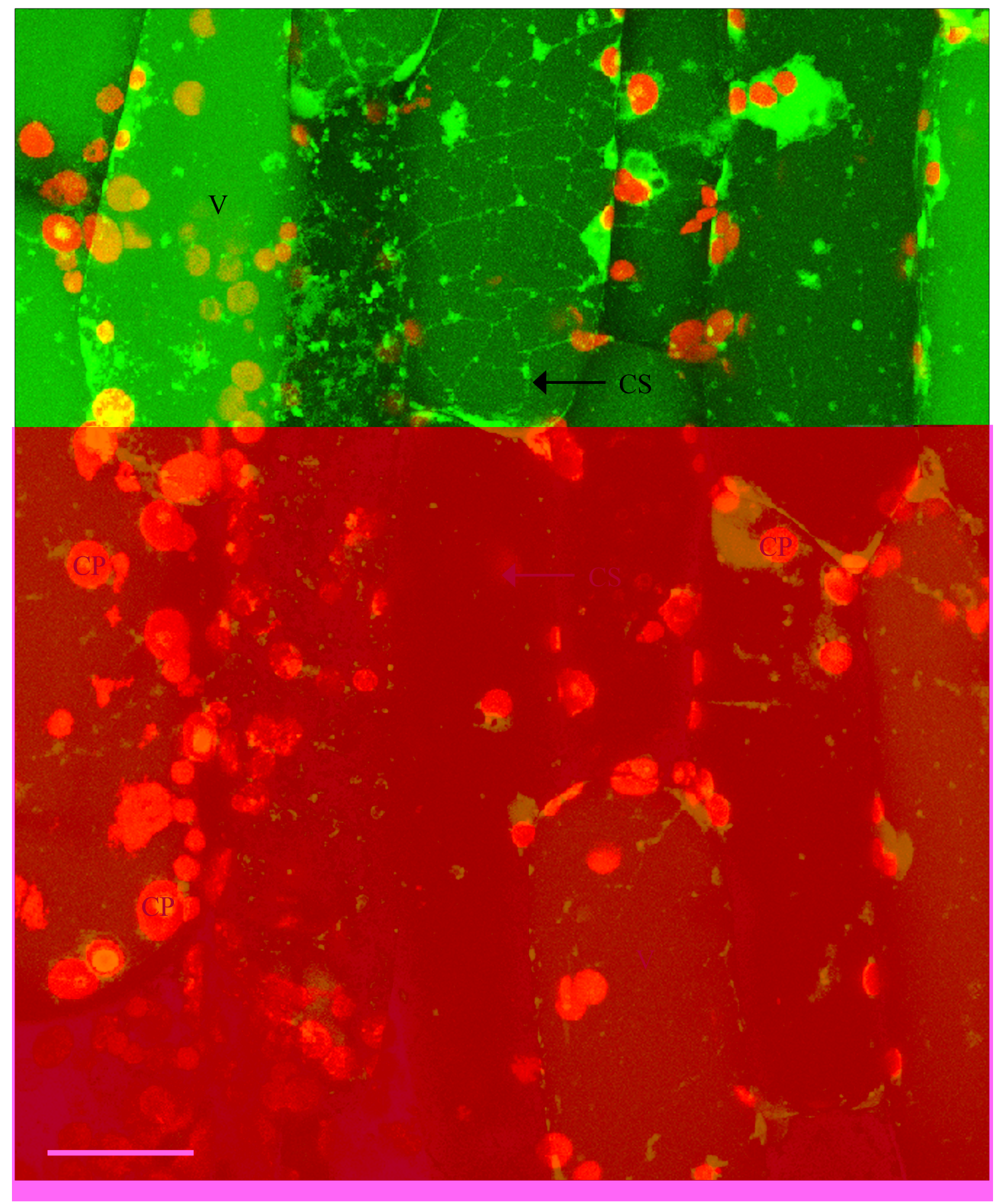

Figure 5.8 Maximum projection of whole L-DOPA fed DOD-6 hypocotyl cells under confocal microscopy. Autofluorescence at $488 \mathrm{~nm}$ excitation with $498-560 \mathrm{~nm}$ emission spectrum for betaxanthins (green) and 625 - $732 \mathrm{~nm}$ emission spectrum for chloroplasts (red). Key: CP chloroplast, CS cytoplasmic streaming, V vacuole. Scale bar $=20 \mu \mathrm{m}$. 

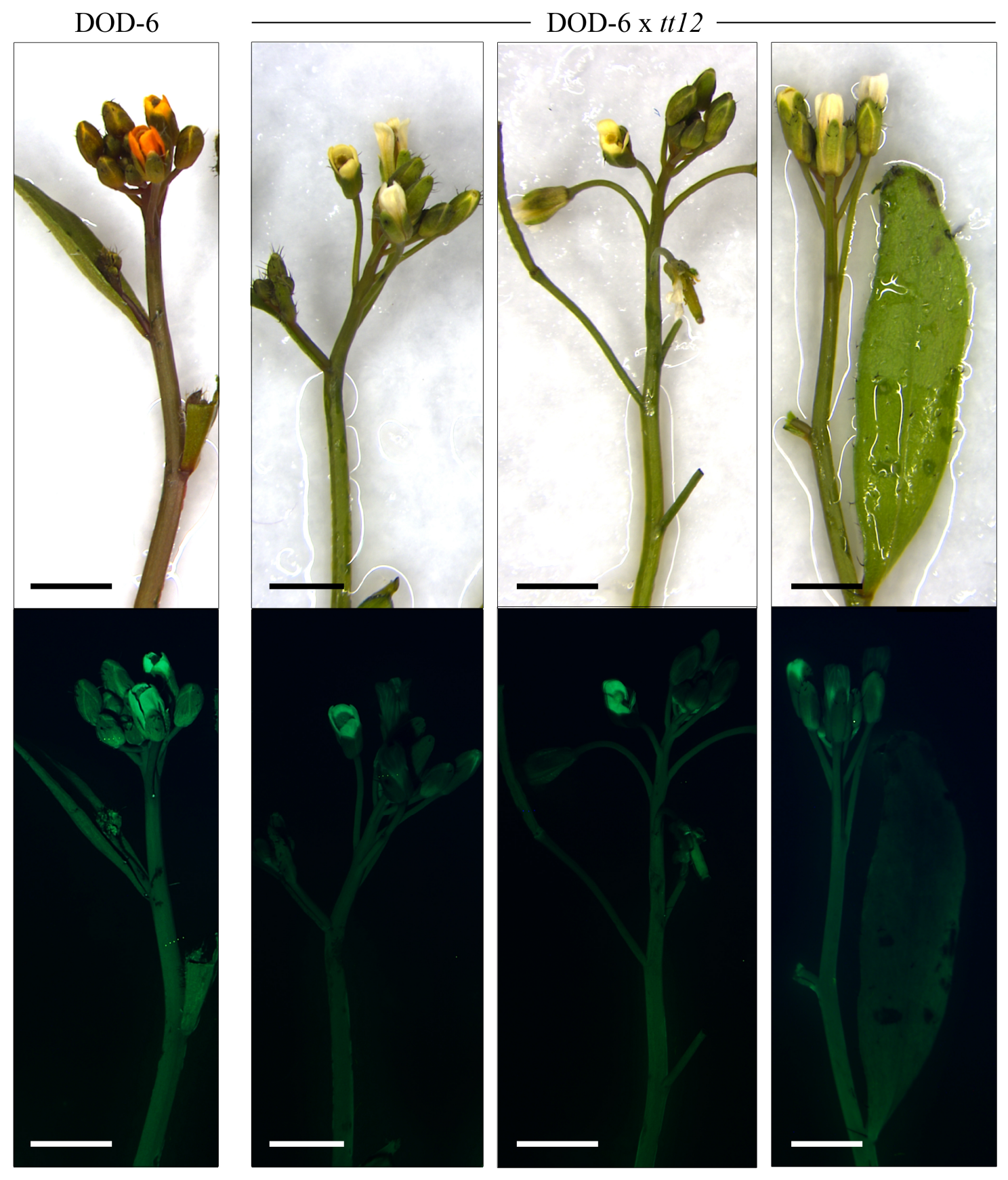

Figure 5.9 Reduced betaxanthin accumulation in L-DOPA fed DOD-6 x tt12 inflorescences. White and blue light images of L-DOPA fed DOD-6 and DOD-6 x tt12. Key: $P$ petal, S sepal. Scale bars $=5 \mathrm{~mm}$. 

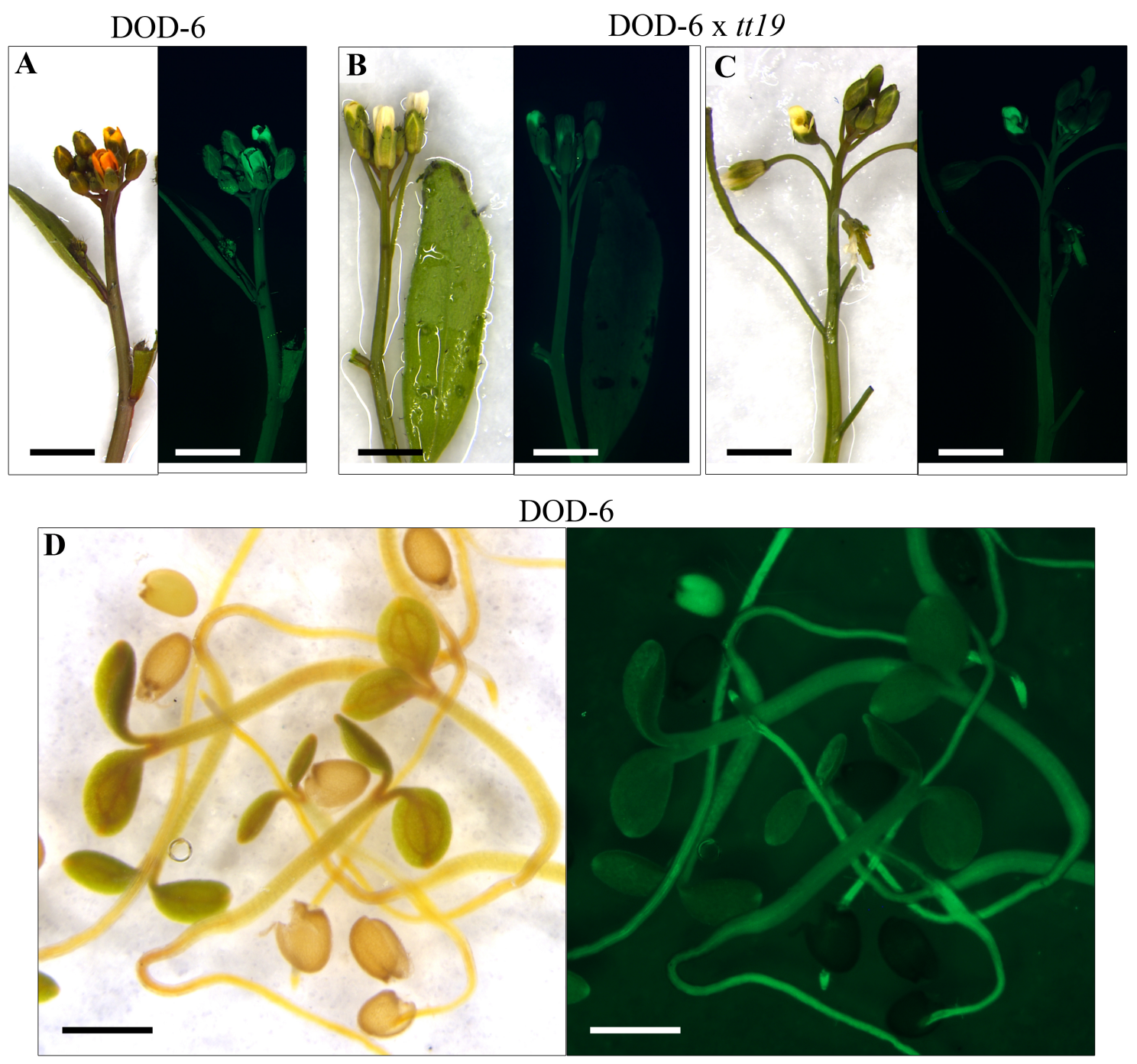

DOD-6 x $t t 19$

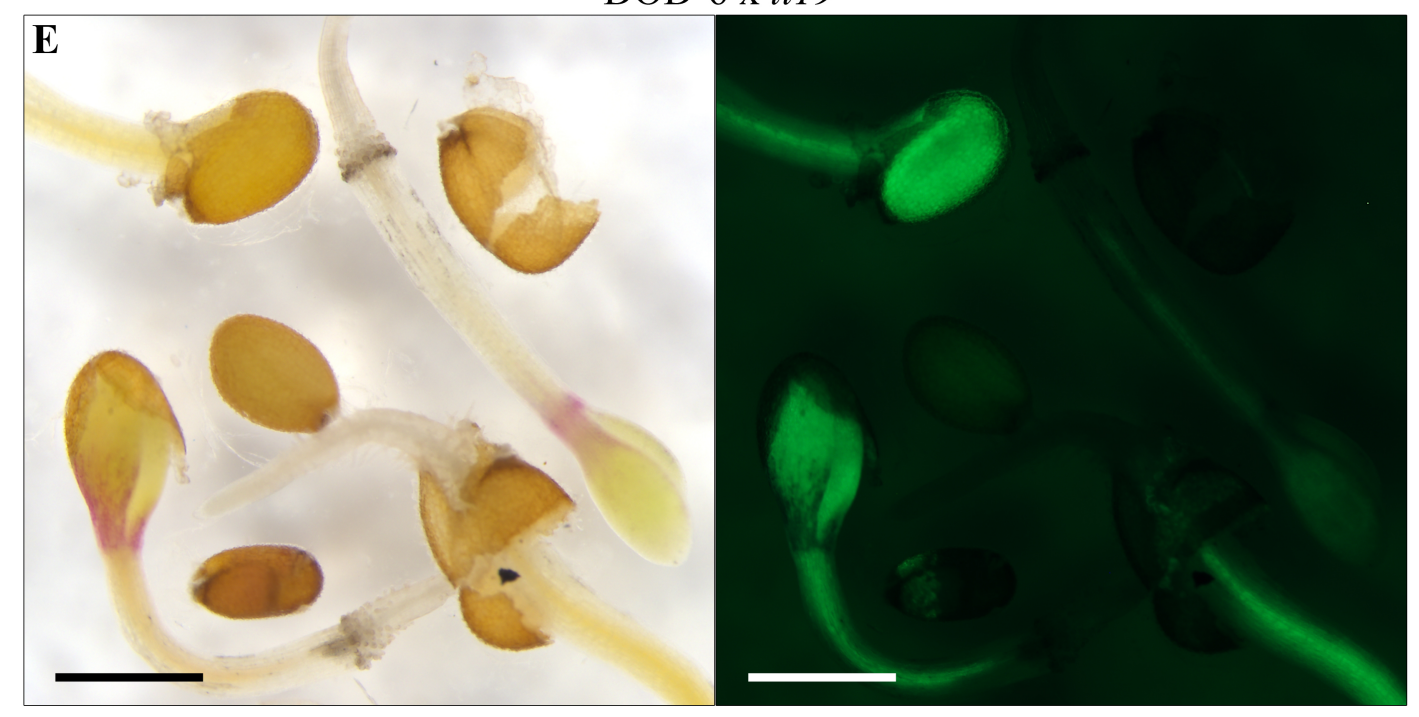

Figure 5.10 Reduced betaxanthin accumulation in L-DOPA fed DOD-6 x tt 19 . White and blue light images of L-DOPA fed (A) DOD-6 and (B and C) DOD-6 x tt19 inflorescences, (D) DOD-6 and (E) DOD-6 x tt19 seedlings. Key: P petal, S sepal. Scale bars: A, $\mathbf{B}$ and $\mathbf{C}=5 \mathrm{~mm}, \mathbf{D}$ and $\mathbf{E}=300 \mu \mathrm{m}$. 

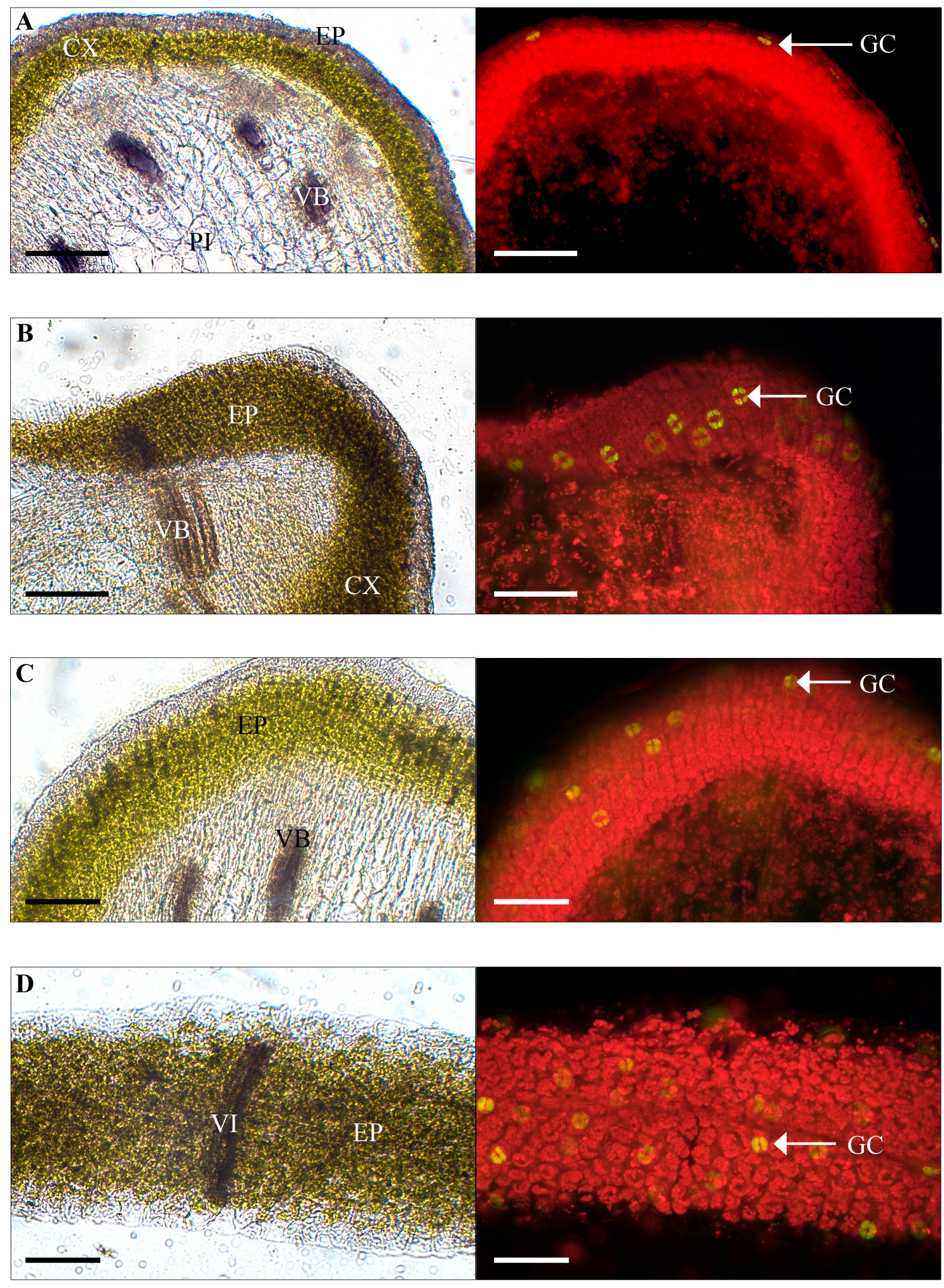

Figure 5.11 Reduced betaxanthin accumulation in L-DOPA fed DOD-6 x tt12 tissues. Transverse sections of (A - C) inflorescence stalks and (D) lamina, under white and blue light. Key: CX cortex, EP epidermis, GC guard cell, PI pith, VB vascular bundle, VI vein. Scale bars $=200 \mu \mathrm{m}$. All sections are unstained. 

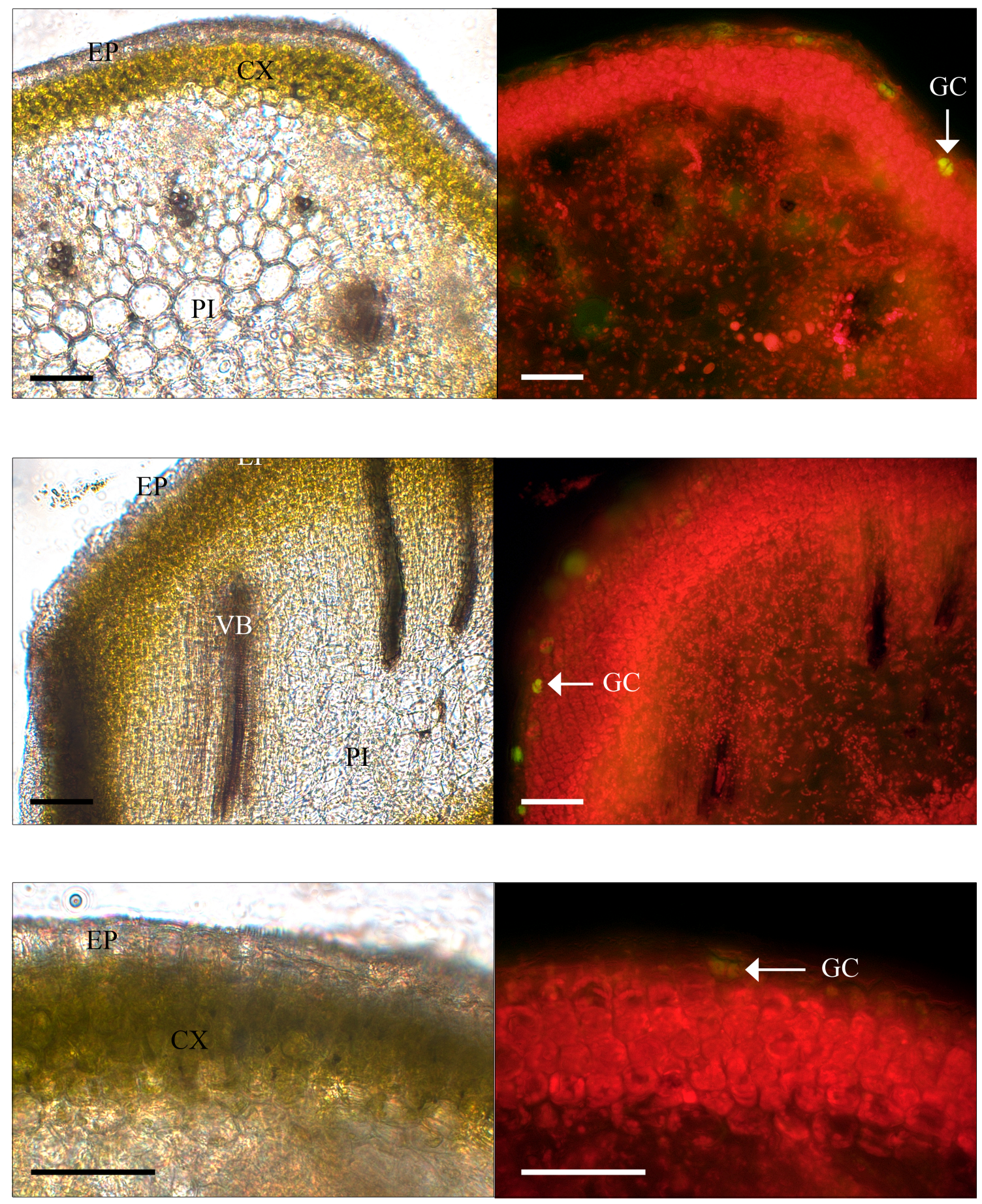

Figure 5.12 Reduced betaxanthin accumulation in L-DOPA fed DOD-6 x $t$ t 19 tissues. Transverse sections of inflorescence stalks under white and blue light. Key: CX cortex, EP epidermis, GC guard cell, PI pith, VB vascular bundle. Scale bars $=100 \mu \mathrm{m}$. 
DOD-6
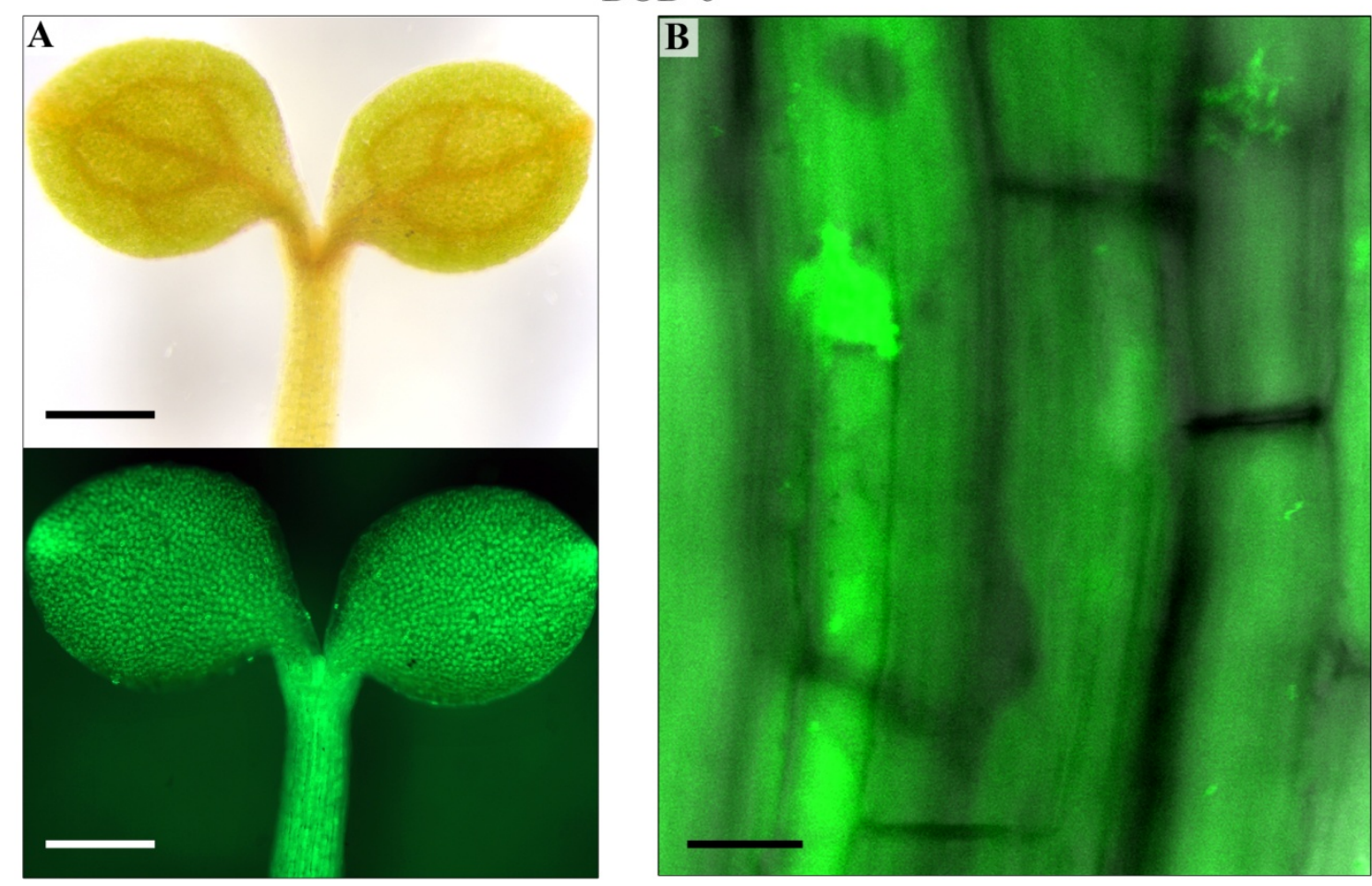

DOD-6 x tt19
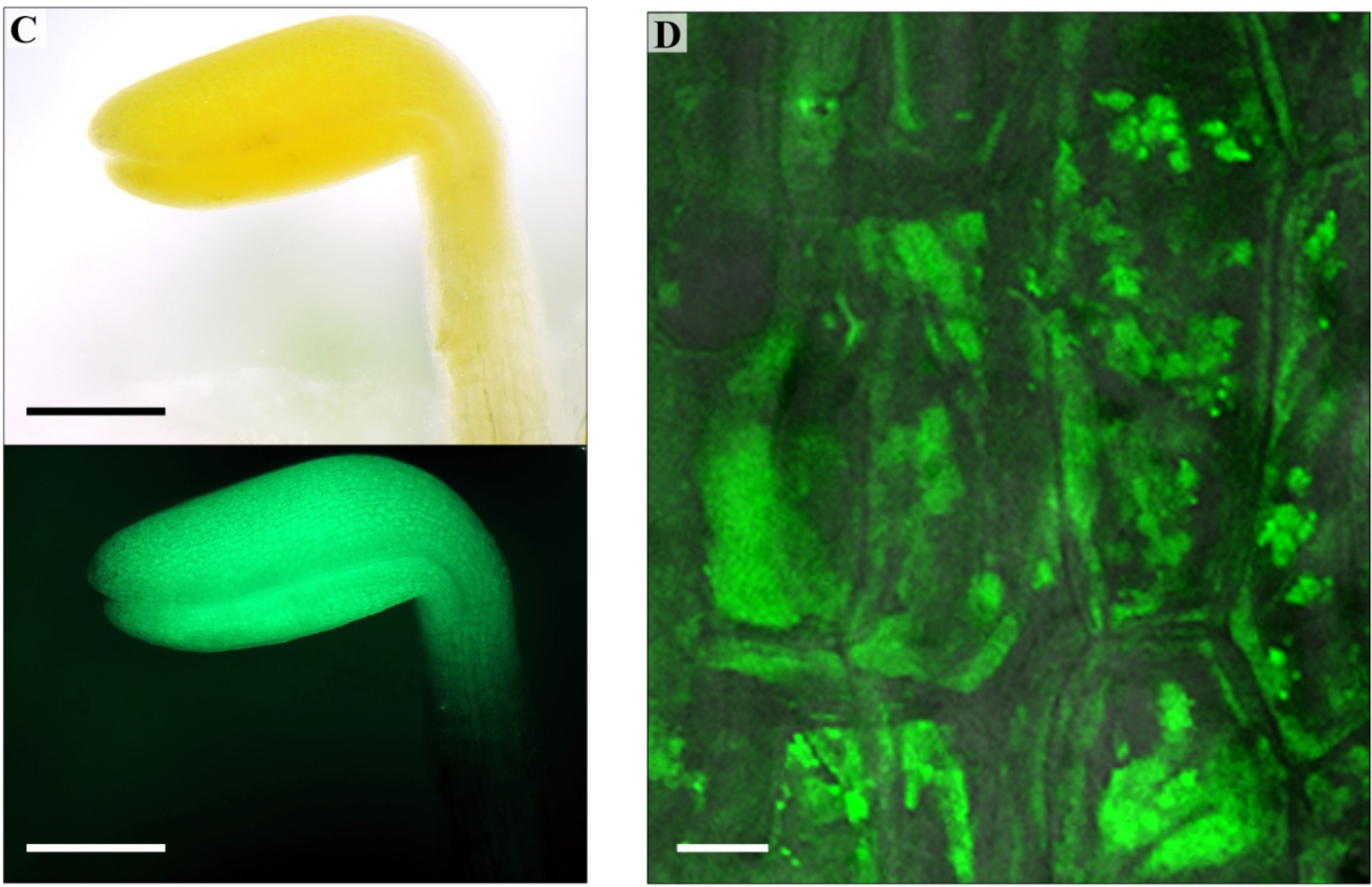

Figure 5.13 Comparison of betaxanthin accumulation in L-DOPA fed (A) DOD-6 with (C) DOD-6 x tt19 seedlings, under white and blue light. Longitudinal section of (B) DOD-6 and (D) DOD-6 x tt19 roots using confocal microscopy. Differential Interference Contrast (DIC) image overlaid with betaxanthin autofluorescence (green) at $488 \mathrm{~nm}$ excitation and $498-560 \mathrm{~nm}$ emission spectrum. Scale bars: $\mathbf{A}=200 \mu \mathrm{m}, \mathbf{C}=$ $300 \mu \mathrm{m}, \mathbf{B}$ and $\mathbf{D}=10 \mu \mathrm{m}$. 


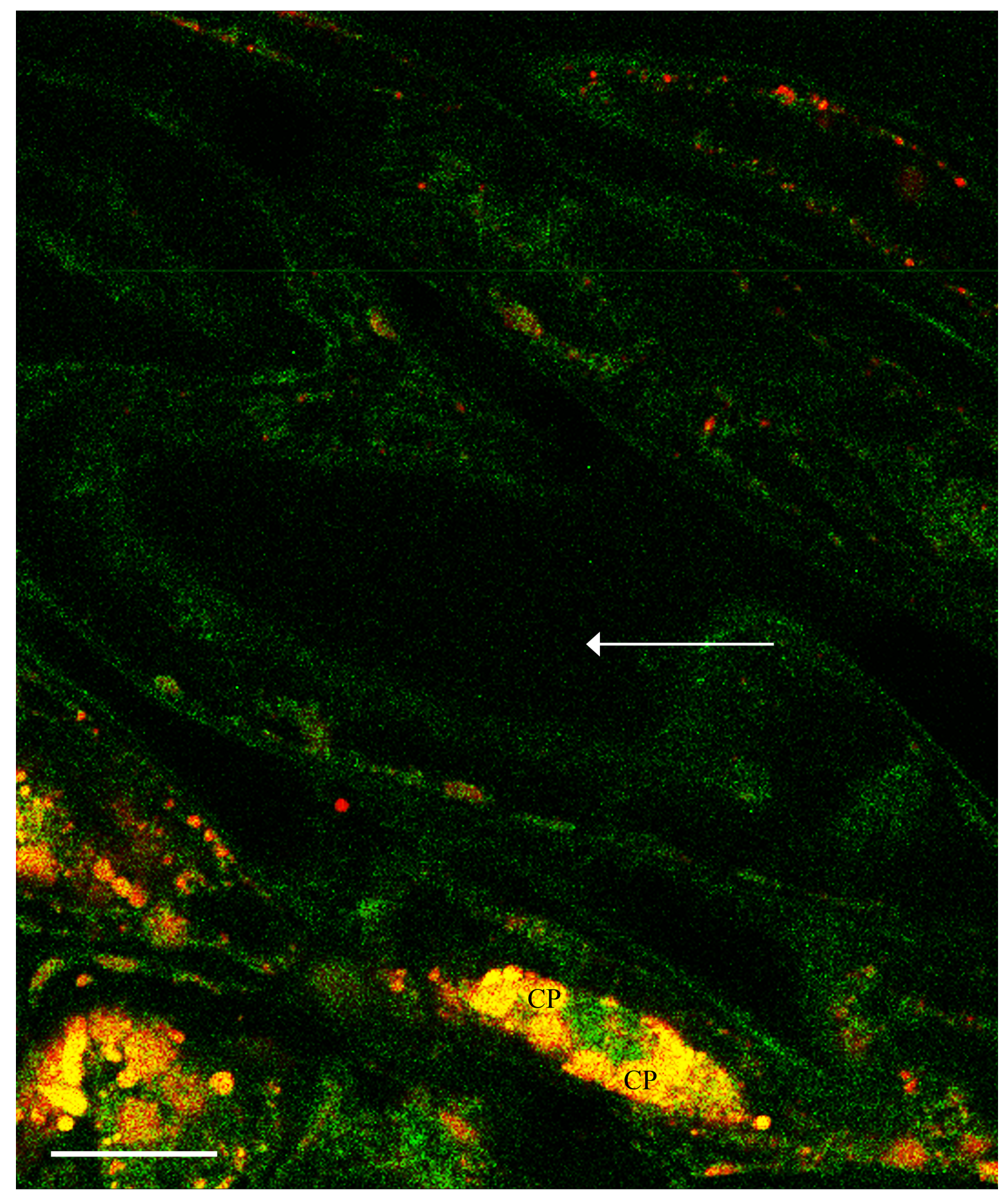

Figure 5.14 Another example of betaxanthin cytoplasmic accumulation in L-DOPA fed DOD-6 x tt19 seedlings. Hypocotyl longitudinal section. Autofluorescence at $488 \mathrm{~nm}$ excitation with 498 - $560 \mathrm{~nm}$ emission spectrum for betaxanthins (green) and 625 $732 \mathrm{~nm}$ emission spectrum for chloroplasts (red). Arrow indicates the vacuole of a single cell. Key: CP chloroplast. Scale bar $=10 \mu \mathrm{m}$. 

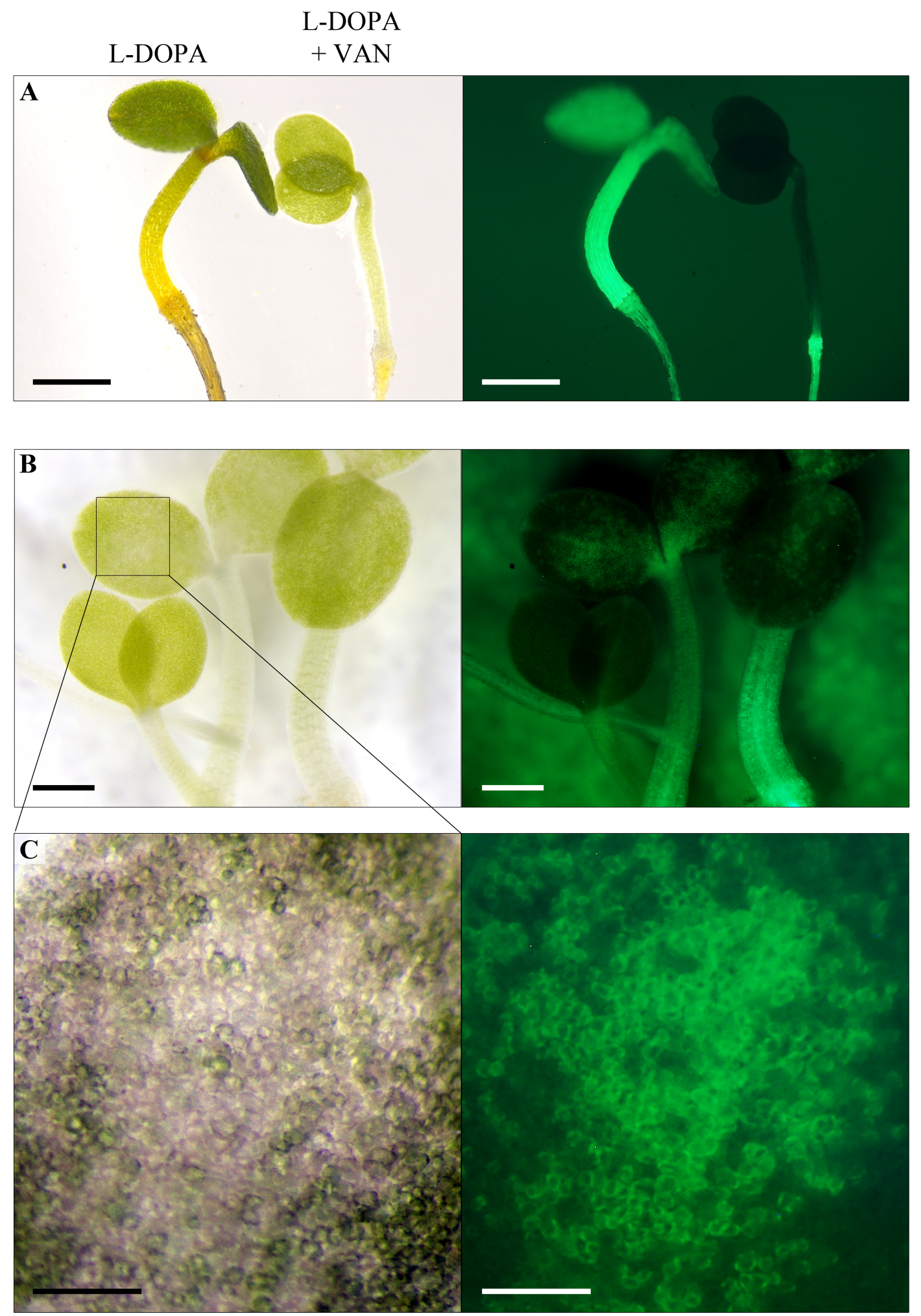

Figure 5.15 Betaxanthin accumulation in vanadate (VAN) treated DOD-6 seedlings under white and blue light. (A) L-DOPA fed DOD-6 (left) and L-DOPA + VAN fed DOD-6 (right). (B) L-DOPA + vanadate fed DOD-6 seedlings with $(\mathbf{C})$ close up of lamina tissue. Scale bars: $\mathbf{A}=800 \mu \mathrm{m}, \mathbf{B}=400 \mu \mathrm{m}$ and $\mathbf{C}=100 \mu \mathrm{m}$. 


\section{L-DOPA}

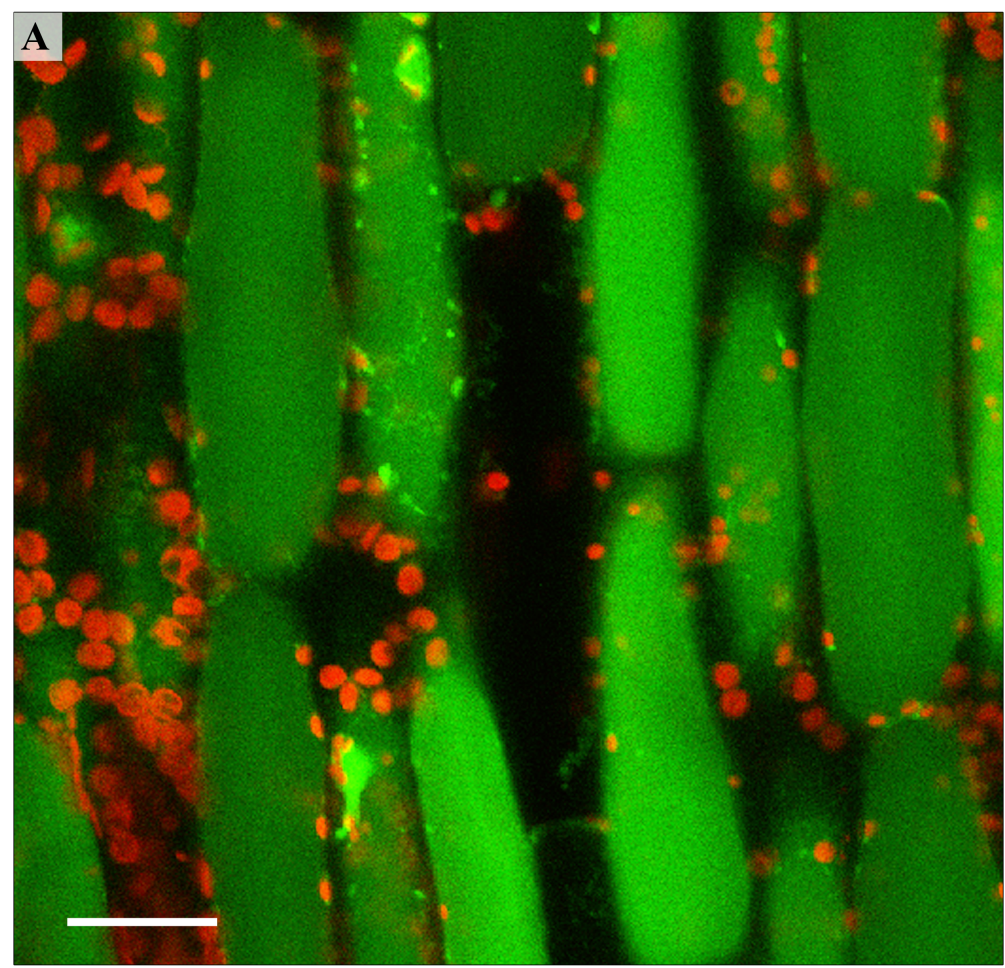

$$
\text { L-DOPA + VAN }
$$

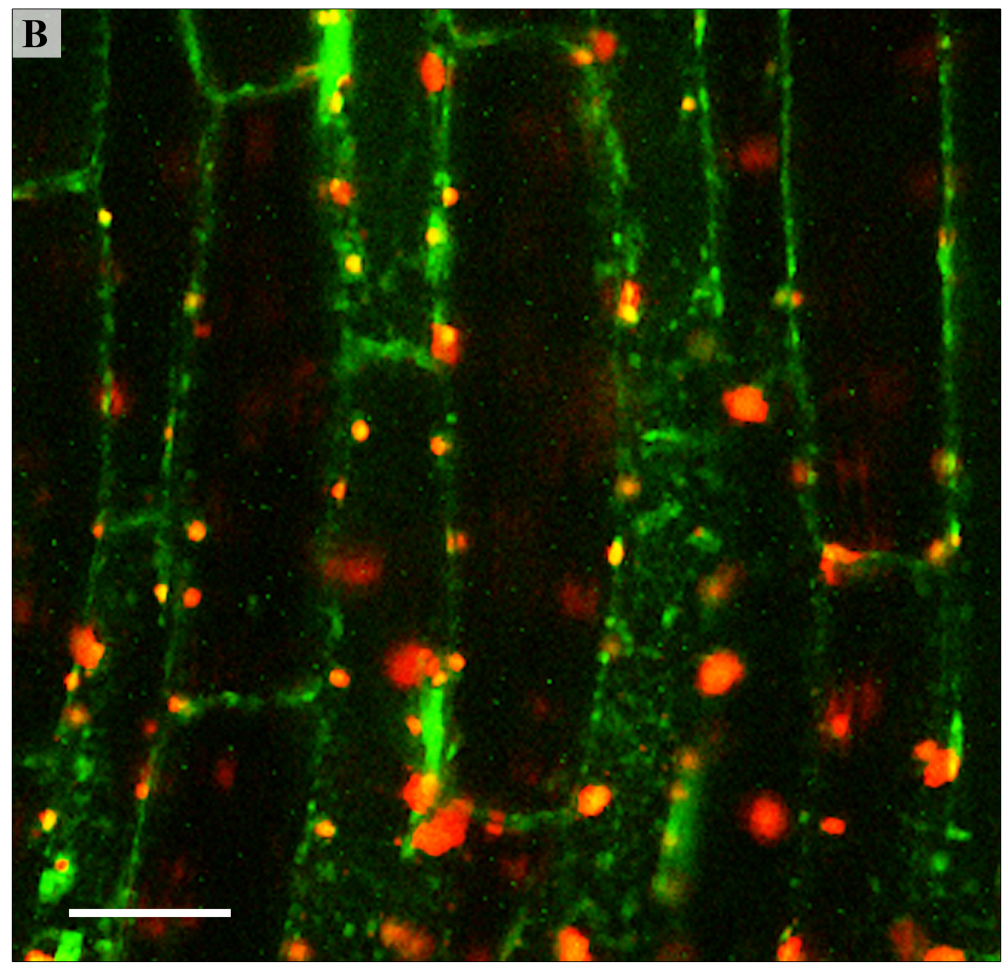

Figure 5.16 Vanadate (VAN) affected betaxanthin accumulation in the vacuoles of L-DOPA fed DOD-6 seedlings. Confocal micrographs of (A) control L-DOPA fed, and (B) L-DOPA + VAN. Autofluorescence at $488 \mathrm{~nm}$ excitation with $498-560 \mathrm{~nm}$ emission spectrum for betaxanthins (green) and $625-732 \mathrm{~nm}$ emission spectrum for chloroplasts (red). Scale bars $=20 \mu \mathrm{m}$. 

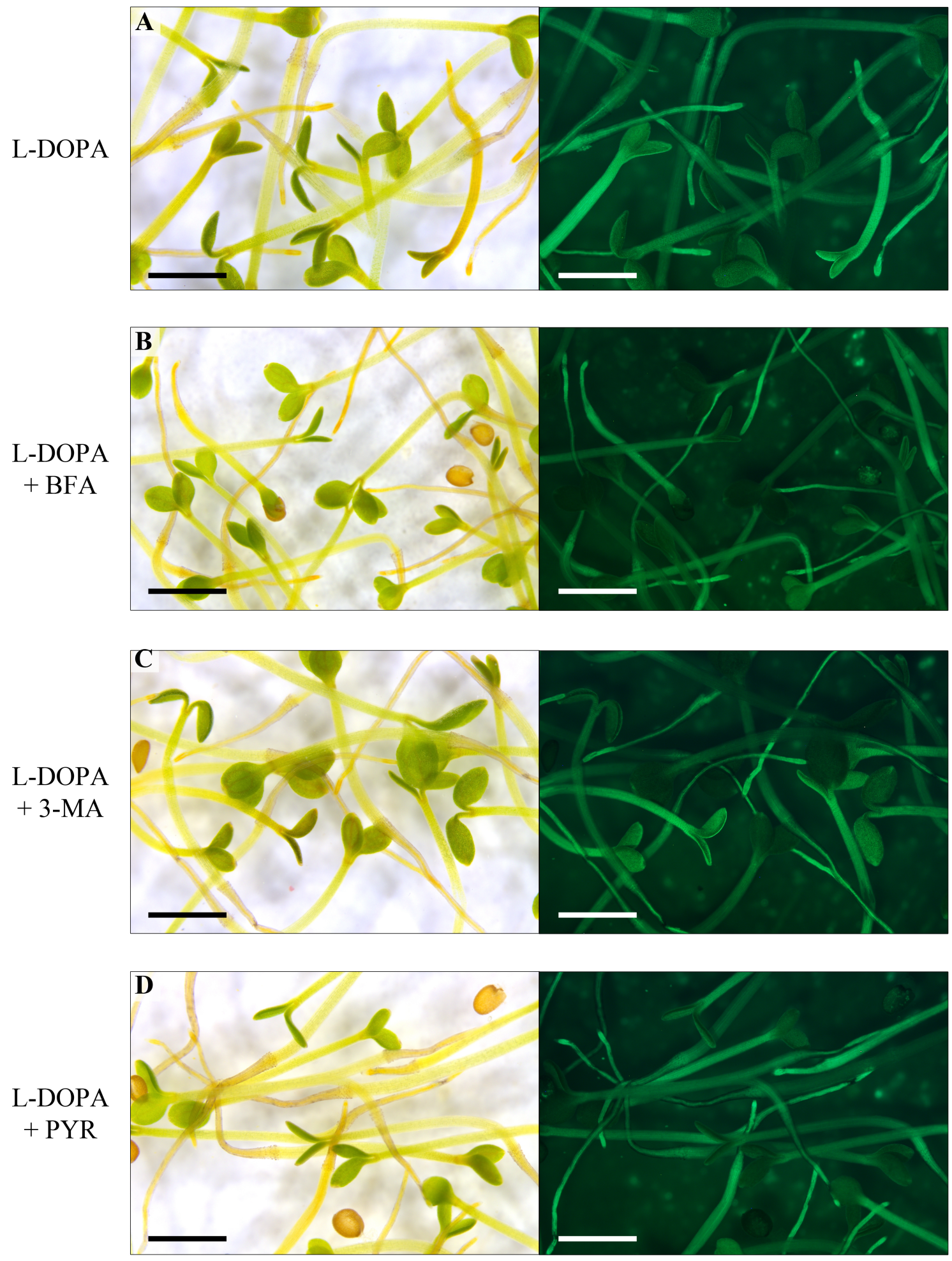

Figure 5.17 Betalain accumulation not affected in DOD-6 fed with L-DOPA and various transport inhibitors, under white and blue light. (A) L-DOPA fed control, (B) BFA treated, (C) 3-MA treated, (D) PYR treated. Scale bars $=1 \mathrm{~mm}$. 

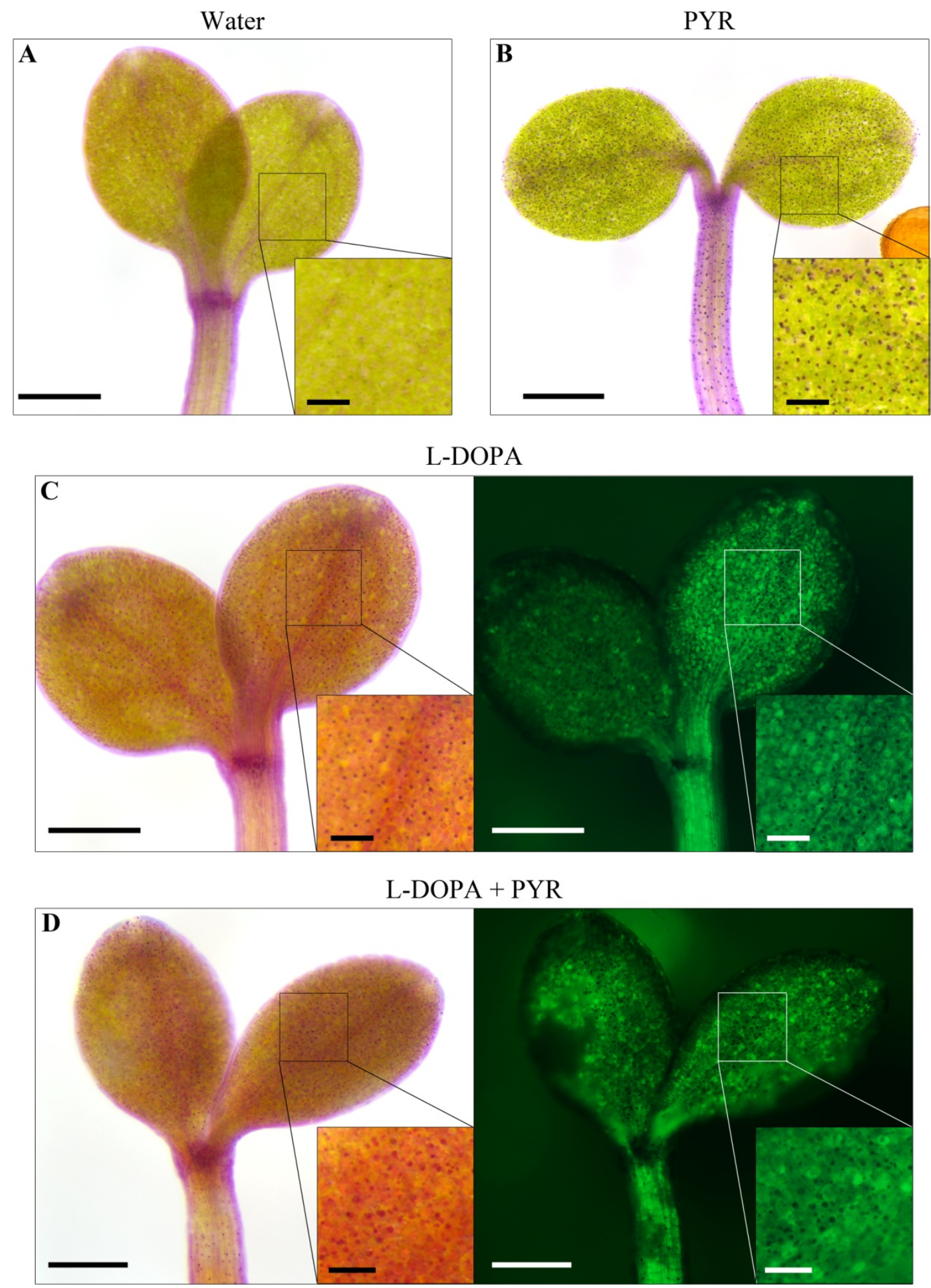

Figure 5.18 DOD-6 x PAP1-5 seedlings treated with pyrimethamine (PYR). White light images of: (A) DOD-6 x PAP1-5 control, and (B) DOD-6 x PAP1-5 treated with PYR. White and blue light images of: (C) DOD-6 x PAP1-5 fed with L-DOPA, and (D) DOD-6 x PAP1-5 fed with L-DOPA plus PYR treatment. Scale bars: A - D = $250 \mu \mathrm{m}$, inserts $=50 \mu \mathrm{m}$. 

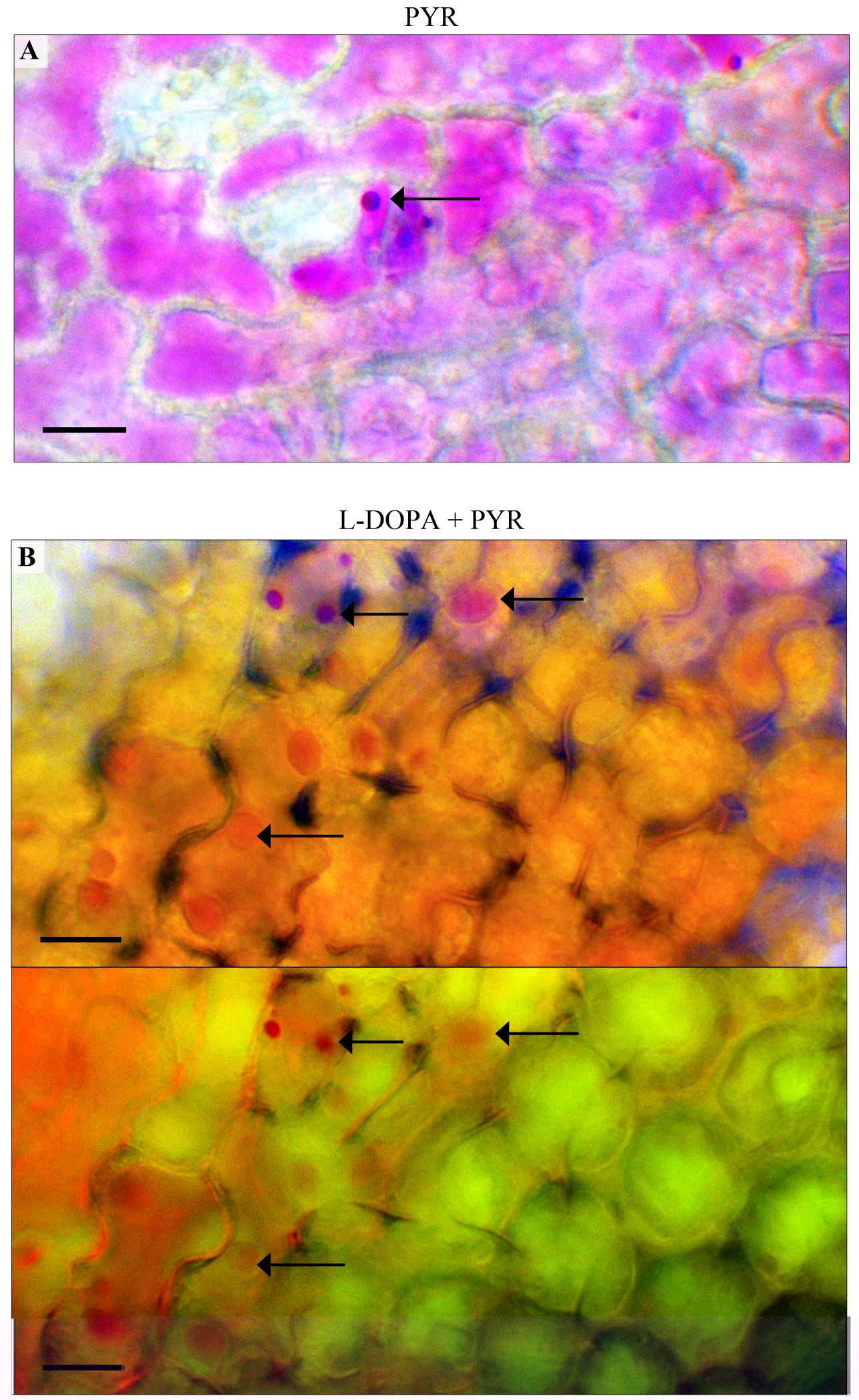

Figure 5.19 Epidermal sections of DOD-6 x PAP1-5 (A) treated with PYR and (B) fed L-DOPA with PYR treatment, under white and blue light. Arrows indicate AVI-like structures. Scale bars $=50 \mu \mathrm{m}$. 


\subsection{Discussion}

\subsubsection{Co-localisation of betalains and anthocyanins}

Creation of the DOD-6 betalain pigment system in Arabidopsis has enabled the first known co-production of anthocyanins and betalains in the same plant. Using this system, it is now possible to test the barriers of anthocyanin and betalain pigments co-existing. It is unknown if physiological barriers exist that prevent both pigments co-localising naturally, such as whether it is viable for plants to produce both pigment types. Using DOD-6 x PAP1-5 plants, the ability for an anthocyanic plant to produce both betalains and anthocyanins in the same tissue was examined. DOD-6 plants when fed with L-DOPA were able to produce pigments in most if not all tissue types throughout the plants (Figure 5.2). This reflects both the $D O D$ is on a $35 S$ promoter, a viral promoter which is constitutively active and maintains high levels of gene expression (Kay et al., 1987) and also a widespread ability of different organs to accumulate betalains. Betaxanthin accumulation was likely only limited by the amount of L-DOPA that reached DOD-6 tissues. In contrast, anthocyanin pigmentation in DOD-6 x PAP1-5, which contains 35S:PAP1 was largely localised to epidermal and vascular tissues (Figure 5.3). Similar tissue localisation was seen when 35S:PAPl was inserted into tomato plants (Zuluaga et al., 2008). It was suggested by Zuluaga et al. (2008) that these tissues contained specific co-factors, such as the bHLH proteins, needed to up-regulate anthocyanin biosynthesis. When L-DOPA was fed to the cross DOD-6 x PAP1-5, betaxanthins strongly accumulated despite the tissue already containing anthocyanins (Figure 5.4). Although betaxanthins and anthocyanins were seen to co-localise in confocal analyses, it is unknown what ratio of betaxanthins to anthocyanins these cells have in comparison to neighbouring cells. Betaxanthin fluorescence in these cells appeared weaker than neighbouring cells without anthocyanins. Through controlled mixing of betaxanthin and anthocyanin extracts, it was observed that betaxanthin autofluorescence can be quenched by the presence of anthocyanins (Figure 5.6). However, in L-DOPA fed DOD-6 x PAP1-5 hypocotyl tissues the anthocyanin-pigmented epidermal cells did not exhibit any green autofluorescence. Co-production of anthocyanins and betalains in DOD-6 suggested that storage limitations may not be the cause. DOD-6 seedlings appeared to produce similar amounts of anthocyanins and betaxanthins compared with production of these 
pigments individually (Figure 5.5). Therefore, signalling mechanisms might exist in certain DOD-6 x PAP1-5 cells which inhibit pigment accumulation in the vacuole when pigment is already present. This would explain why hypocotyl epidermal cells that contained anthocyanins prior to the feeding of L-DOPA appeared to lack betaxanthins. This is an area that requires further evaluation.

\subsubsection{Betalain transport}

No studies on possible betalain transport mechanisms have been published. In this chapter, known anthocyanin transport mechanisms were tested for their involvement in betalain accumulation. Experiments were carried out using DOD-6 Arabidopsis, as well as with known anthocyanin transport mutants, $t t 19$ and $t t 12$, and with transport inhibition assays. The findings from these analyses are illustrated in Figure 5.20. Betalain transport is likely to be direct from site of synthesis on the endoplasmic reticulum (ER) to storage in the vacuole. Brefeldin A (BFA), a Golgi-disturbing agent (Dinter \& Berger, 1998), was used to test whether betaxanthins pass through the trans Golgi network (TGN) before vacuolar accumulation. BFA treatment in L-DOPA fed DOD-6 appeared to have no affect on betaxanthin accumulation, based on visual assessment of autofluorescence and colour (Figure 5.17B). These results suggest a Golgi-independent transport route for betalains (Figure 5.20A), similar to that of anthocyanins (Poustka et al., 2007).

Vesicular transport seems involved in betaxanthin transport (Figure 5.20B). Confocal analyses of DOD-6 during the accumulation of betaxanthins after the feeding of L-DOPA revealed a large number of small vesicle-like structures clustering in the cytoplasm (Figure 5.7). In addition, these structures were in string-like formations across the cells (Figure 5.8), which may be vesicles carried by cytoplasmic streaming. Vesicles and other organelles can be carried on microfilaments by myosins. Cytoplasmic streaming of vesicles containing betalains would enable rapid movement of these pigments across the cell. An interesting experiment would be feeding the myosin inhibitor 2, 3-butanedione monoxime (BDM; Verchot-Lubicz and Goldstein (2010)), to see whether cytoplasmic streaming is essential for betaxanthin accumulation in the vacuole. In addition to these vesicular-like structures, larger circular compartments containing betaxanthin autofluorescence were observed. 


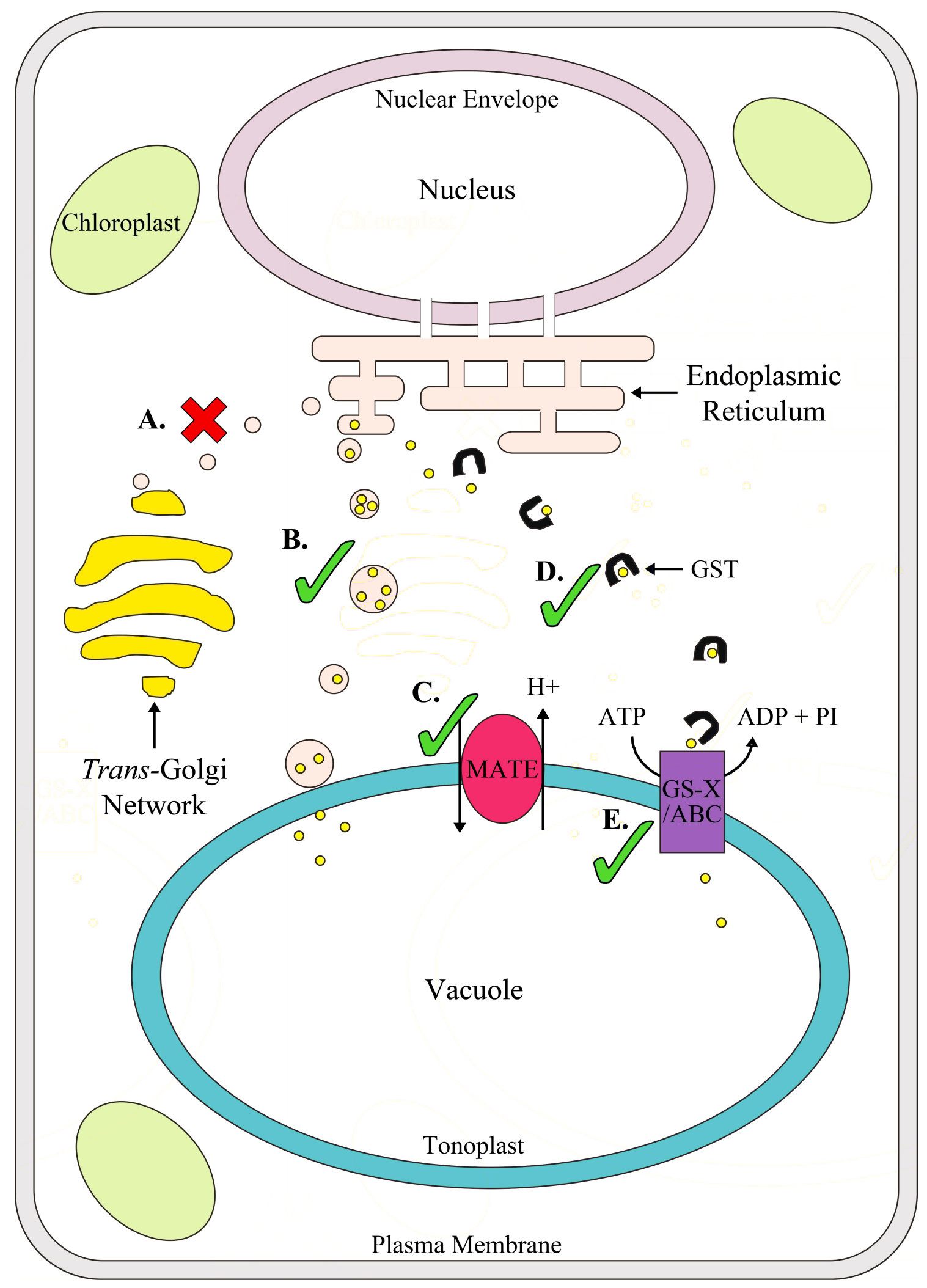

Figure 5.20 Illustration of the results from the betalain transport experiments. (A) trans-Golgi network-mediated transport route; (B) Vesicular transport; (C) Multidrug and toxic extrusion (MATE) transporter; (D) Glutathione $S$-transferase (GST) carrier proteins; (E) Glutathione/ATP-binding cassette (GS-X/ABC) transporter. The red cross indicates that the mechanism is unlikely and the green tick indicates that there is evidence to support this transport mechanism. 
These larger compartments appeared to either be budding or merging, and may be part of vacuole biogenesis. Vacuolar biogenesis begins at cell division in the shoot or root apical meristem (Marty, 1999; Taiz, 1992). Meristematic cells contain hundreds of small membrane-bound structures called provacuoles, which arise by the budding and fusion of vesicles from the TGN and the ER (Staehelin, 1997). During plant cell expansion, these provacuoles gradually fuse to form the central vacuole. The role of autophagy in vacuole biogenesis and betaxanthin accumulation was then tested. Autophagy is a mechanism used by compounds and proteins for vacuolar deposition (Kulich et al., 2013; Robinson et al., 1998). These compounds or proteins released by the ER are surrounded by ER-derived vesicles which then fuse before transporting the substrate to the vacuole. Autophagy inhibitor, 3-MA, did not visibly disrupt or modify betaxanthin accumulation in L-DOPA fed DOD-6 (Figure 5.17), which suggests that their transport does not require autophagy. Similar results were found when anthocyanin-producing Arabidopsis were treated with 3-MA (Poustka et al., 2007).

Cellular images of betaxanthin accumulation also gave us insight into betalain biosynthesis. Previously, the conjugation reaction of betalamic acid with amines/amino acids to form betaxanthins was said to occur in the vacuole. Whereas, the conjugation of betalamic acid and cyclo-DOPA to form betacyanin occurred in the cytoplasm (Trezzini \& Zrÿd, 1989). However, betalamic acid is a non-fluorescent compound (GandíaHerrero et al., 2010; Gandía-Herrero et al., 2005b), which means that the green autofluorescence seen in these sections must be betaxanthins. Therefore, the conjugation reactions to form betaxanthins must occur before deposition in the vacuole, at least in this artificial model system. It would be important to analyse these betalain compounds to confirm their identity.

The next transporter tested for involvement in betalain transport was the MATE transporter, using the Arabidopsis mutant $t t 12$. Results suggest that this transporter is involved in betaxanthin accumulation (Figure 5.20C). From visual assessments, L-DOPA fed DOD- 6 x $t$ t 12 crosses appeared to have reduced levels of betaxanthins accumulating in tissues (Figure 5.9). In sections of the flower stalks, TT12 appeared to have a strong effect on betaxanthin accumulation, as remaining autofluorescence was only seen in the guard cells (Figure 5.11). TT12 is part of a large MATE gene family in Arabidopsis with 56 members (Hvorup et al., 2003) and uses a $\mathrm{H}^{+}$gradient to transport 
substrates across membranes. Vacuolar transport is essential for the function of guard cells (Grabov \& Blatt, 1998; Schroeder et al., 2001) and recently, a gene for a specific flavonoid MATE transporter, FFT, was found to be expressed in guard cells of Arabidopsis (Thompson et al., 2010). Therefore, betaxanthin accumulation in guard cells may not have been affected by the $t t 12$ mutation due to the presence of this other MATE. Although not further analysed, the TT12 MATE transporter appears to be essential in most cell types for betaxanthin transport in Arabidopsis. AtTT12 is not essential for anthocyanin vacuolar accumulation, only that of proanthocyanidins (Debeaujon et al., 2001; Marinova et al., 2007). However, in the study by Poustka et al. (2007), anthocyanin accumulation in Arabidopsis was affected by treatment with pyrimethamine (PYR), a MATE inhibitor. These tissues maintained similar levels of anthocyanins to the control, but had an increase in anthocyanic vacuolar inclusions (AVIs). Given that $t t 12$ did not affect anthocyanin accumulation and PYR did, it is possible that Arabidopsis has a specific MATE transporter for proanthocyanidins and one for anthocyanins. In Vitis vinifera (grape) two MATE transporters are involved in anthocyanin transport (Gomez et al., 2011). In Arabidopsis, PYR might be acting upon a second, unidentified MATE. L-DOPA fed DOD-6 treated with PYR did not appear to have affected betalain accumulation (Figure 5.17). In DOD-6 x PAP1-5, however, PYR induced AVI-like structures to form. It was hypothesised by Poustka et al. (2007) that PYR inhibits the fusion of anthocyanin-containing vesicles with the central vacuole, thus promoting AVI formation. A similar structure to AVIs has been reported in betalain-producing plants (Iwashina et al., 1988). Although no evidence was seen of a similar occurrence in DOD-6 treated with PYR (data not shown), in DOD-6 x PAP1-5 the presence of betaxanthins in the AVI-like structures appeared possible (Figure 5.19). Chemical analysis of these putative betaxanthin- and anthocyanin-containing structures would be important to know what compounds they contain. Some types of AVI are extractable from the cell, allowing for chemical analysis (Conn et al., 2010).

GST-like transporters are another mechanism that betaxanthins appear to use for vacuolar accumulation (Figure 5.20D). Similar to the results for DOD-6 x $t$ t12 plants, L-DOPA fed DOD- 6 x $t$ t19 had visibly reduced autofluorescence when compared with the DOD-6 control (Figure 5.10). The only cells appearing to accumulate betaxanthins were guard cells (Figure 5.12). Like in L-DOPA fed DOD-6 x tt12 tissues, the betaxanthin autofluorescence in guard cells could have been due to the presence of 
redundant transport pumps. In L-DOPA fed DOD-6 x $t t 19$ tissues, confocal analyses revealed blotchy autofluorescence in cytoplasm with no vacuolar accumulation (Figures 5.13 and 5.14). These results suggest that GST-like transport is essential for betaxanthins to reach the vacuole in most tissues. GSTs are primary transporters that directly use ATP to energise uphill transport into the vacuole from the cytosol (Etxeberria et al., 2012). GSTs usually tag the substrate with a glutathione molecule for recognition at the associated glutathione pump (GS-X pump) on the tonoplast (Marrs, 1996; Martinoia et al., 1993). GST-like proteins have been linked anthocyanin transport in a number of species (Alfenito et al., 1998; Kitamura et al., 2012; Kitamura et al., 2004; Marrs et al., 1995). Anthocyanin accumulation could be restored to plant lines carrying mutations of those GSTs by introduction of GST from another species. This suggests that GSTs fulfil similar roles in anthocyanin transport for each species (Koes et al., 2005). However, proanthocyanidins were not restored in Arabidopsis tt19 when a GST from Petunia (AN9) was introduced (Kitamura et al., 2004). This may mean that anthocyanins and proanthocyanidins have overlapping yet distinct GST transport pathways. The precise function of GSTs in anthocyanin transport is still unknown. It is conceivable that these proteins tag anthocyanins with glutathione molecules. However, there is no evidence of anthocyanin-glutathione conjugates naturally occurring in vivo (Francisco et al., 2013; Mueller et al., 2000). Another suggested function for GSTs in flavonoid transport is that GST-like transporters directly bind to anthocyanin and proanthocyanidin molecules as carrier proteins or 'molecular chaperones' (Mueller et al., 2000). For Arabidopsis tt 19 mutants, proanthocyanidins accumulated in vesicle-like structures, suggesting that AtTT19 has a role in transport of the vesicles to the central vacuole (Kitamura et al., 2004). Although TT19 proteins do not possess vacuolar transport signals (Kitamura, 2006), TT19 proteins were found localised to the cytosol and surrounding small vacuolar-like structures (Kitamura et al., 2010). Blotchy betaxanthin autofluorescence seen in DOD- 6 x $t t 19$ cells suggest that, similar to proanthocyanidins in $t t 19$, vesicle-like structures containing betaxanthins cannot reach the central vacuole without functioning GST-like proteins. Staining of cellular compartments would help elucidate what these structures are, and how transport is affected.

An ATPase may be involved in betalain accumulation (Figure 5.20E). Betaxanthin accumulation was visibly reduced in vanadate (VAN) treated DOD-6 seedlings fed with 
L-DOPA (Figure 5.15). Confocal analyses of this tissue suggested that VAN reduced vacuolar accumulation of betaxanthins (Figure 5.16). VAN is a general ATPase inhibitor and is thought to be directly impairing ATP-binding cassettes (ABCs) on the tonoplast (Goodman et al., 2004; Theodoulou, 2000), in particular GS-X pumps. ABC/GS-X transporters have been implicated in flavonoid transport in a number of species (Francisco et al., 2013; Goodman et al., 2004; Lu et al., 1997; Rosado et al., 2011). The treatment of anthocyanin-producing plants with VAN, reduced anthocyanin vacuolar accumulation in Zea mays (maize; Marrs et al. (1995)), and enhanced AVI formation in Arabidopsis (Poustka et al., 2007). The different responses of maize and Arabidopsis to VAN suggest that the involvement of ATPases in anthocyanin transport may be compound or species dependent. The treatment of DOD-6 plants with vanadate had a dramatic effect upon betaxanthin accumulation, indicating that an ATPase may play a key role in betalain transport.

\subsubsection{Conclusions and future analyses}

During evolution of the production of the plant pigments, anthocyanins and betalains, it is unknown why plants evolved to produce one type without the other. This chapter demonstrated for the first time that betalains and anthocyanins can co-exist in the same tissues. Therefore contrary to my hypothesis, lethality does not appear to be the issue, and mutual exclusivity could have resulted from redundant functions as proposed in the replacement hypothesis. In support of this hypothesis, betaxanthins in the artificial betalain pigment model, DOD-6, were found to share many of the same transport mechanisms as anthocyanins. However, some aspects of the transport of betaxanthins differed from anthocyanins, given that the proanthocyanidin TT12 MATE transporter may be involved for betaxanthins. The effect upon anthocyanin accumulation by the MATE inhibitor PYR, does suggest that another MATE protein might be involved in anthocyanin transport. This study reveals that many details of betalain and anthocyanin transport are still to be discovered. Especially the role of each proposed mechanism and how they fit together. Many experiments can be pursued from this data and a few of these are outline below.

- Investigating the transport of betacyanins with the DOD-6/CYP76AD1 Arabidopsis line to see whether they use the same mechanisms as betaxanthins.

- Staining of cellular membranes in L-DOPA fed DOD-6 for the identification of 
the vesicle-like structures.

- Characterisation of TT12 and TT19 transport mechanisms in DOD-6 x $t t$ crosses.

- Chemical transport inhibition assays with betalain-producing plants to test whether the same transport mechanisms are used.

- Investigate where the actual conjugation reactions of betalains occur and how this may control betalain biosynthesis and distribution.

Transport appears to be a complex story for both betalains and anthocyanins. The research so far strongly supports a direct replacement of betalains and anthocyanins. Betalains accumulate in similar tissues, are induced by similar environmental factors and may use similar transport mechanisms. It indicates that only a few genetic changes were required to evolve betalain production in plants, with many of the previously existing anthocyanin pathway components (such as transport) able to be co-opted for the new pigments. The next chapter will explore biosynthetic regulation of betalain pigments, and whether betalains are controlled by the same regulatory mechanisms as flavonoids. 


\section{Chapter 6: Betalain biosynthetic control}

\subsection{Introduction}

Although many studies have investigated betalain biosynthesis, little is known about how the betalain biosynthetic pathway is regulated (Gandía-Herrero \& García-Carmona, 2013). Genes in the flavonoid biosynthetic pathway are regulated by a complex mix of transcription elements which include R2R3MYBs and basic helix-loop-helix (bHLH) transcription factors with WD40-type proteins and their interaction (reviewed by Davies and Schwinn (2003); Koes et al. (2005); Mol et al. (1998); Tanaka et al. (2008)). As discussed in Section 1.5, biosynthesis of betalain and anthocyanin pigments is induced by many of the same external factors, such as physical wounding (Gould et al., 2002; Sepúlveda-Jiménez et al., 2004). Betalains and anthocyanins also have similar pigmentation patterns in vegetative tissues (Chapter 3), and may use similar vacuolar transport mechanisms (Chapter 5). Therefore, it is possible that during the evolution of betalain pigments in plants, betalain biosynthesis incorporated the use of the anthocyanin regulatory system.

The betalain pathway is simple in comparison with the flavonoid pathway (Grotewold, 2006). Only three main enzymatic steps have been elucidated (Figure 6.1). These steps are, [1] the conversion of, L-tyrosine to DOPA by a tyrosine hydroxylating enzyme (Gandía-Herrero et al., 2005a; Gandía-Herrero et al., 2009; Steiner et al., 1999; Wang et al., 2007; Yamamoto et al., 2001), [2] production of betalamic acid from DOPA by 4,5-DOPA extradiol dioxygenase (DOD) (Christinet et al., 2004; Gandía-Herrero \& García-Carmona, 2012; Mueller et al., 1997a; Sasaki et al., 2009; Sekiguchi et al., 2010; Takahashi et al., 2009; Zhao et al., 2011), and [3] the formation of cyclo-DOPA by cytochrome P450 (CYP76AD1) (Hatlestad et al., 2012). Apart from the secondary modifications of glycosylation (Das et al., 2013; Heuer et al., 1996) and acylation (Bokern et al., 1992; Bokern \& Strack, 1988), the remaining steps are all spontaneous processes (Hempel \& Böhm, 1997; Schliemann et al., 1999; Strack et al., 2003). Given the few enzymatic steps, biosynthetic regulation of betacyanins may be through the control of cyclo-DOPA synthesis, while control of tyrosine or DOPA synthesis would control production of both types. However, as discussed in Section 3.4.2, adjacent tissues of some species including Swiss chard (Beta vulgaris subsp. cicla cv. 'Bright 


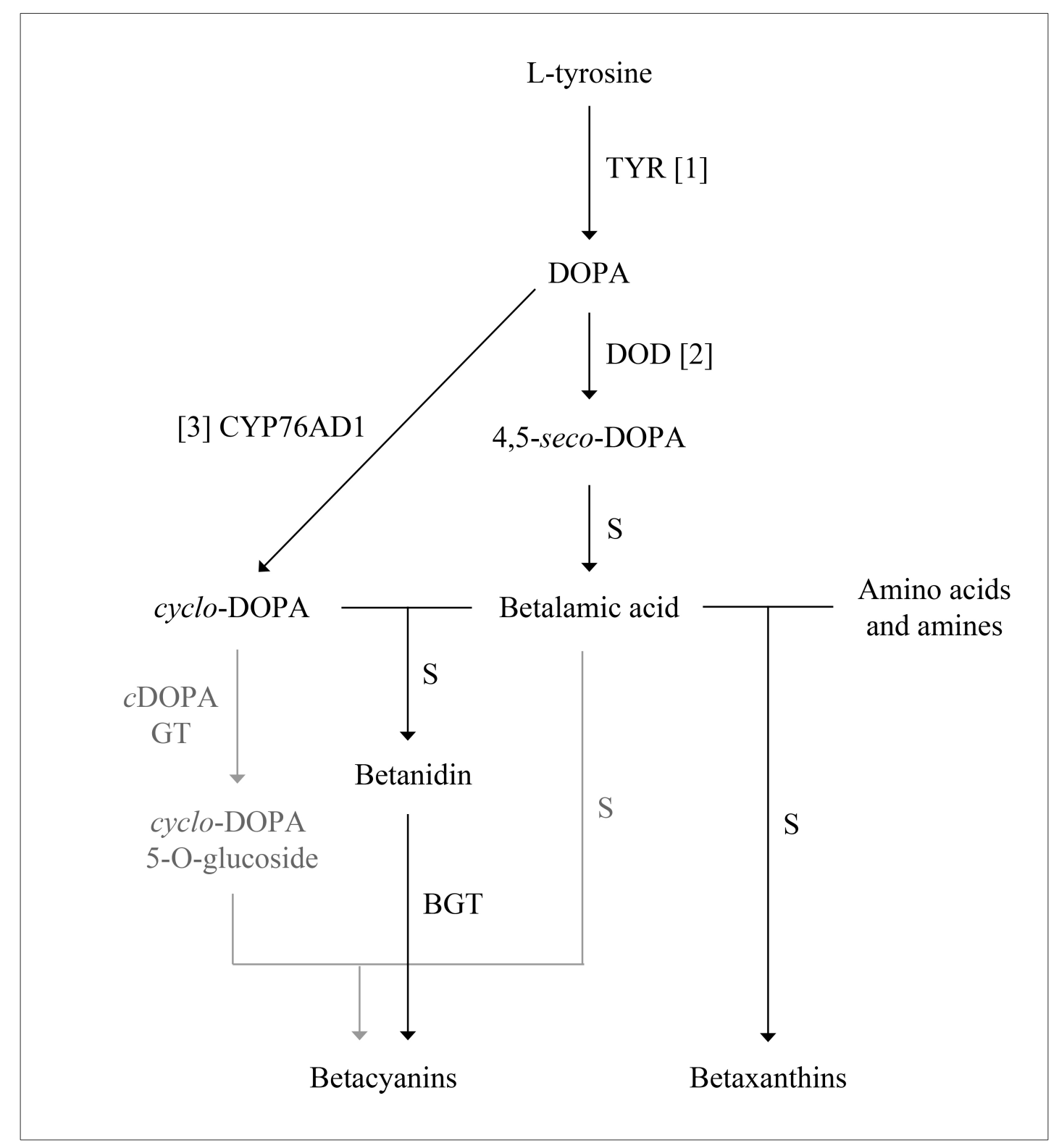

Figure 6.1 Betalain biosynthetic pathway. Enzyme code (numbers referred to in text): BGT betanin glycosyltransferase cDOPA GT cyclo-DOPA glycosyltransferase, CYP76AD1 cytochrome P450, DOD 4,5-DOPA extradiol dioxygenase, TYR tyrosinase. Grey indicates possible alternative glycosylation pathway. Steps labelled $\mathrm{S}$ are spontaneous reactions. 
Lights') have discrete control over the ratio of betaxanthins to betacyanins produced. Investigations into biosynthetic regulation in Beta vulgaris cell cultures (Girod \& Zrÿd, 1991; Leathers et al., 1992; Mukundan et al., 1999; Vincent, 1994; Zrÿd et al., 1982) suggest three possible points of control in the pathway: (1) substrate limitation, (2) enzyme synthesis and/or activity, (3) two separate tyrosine pools for cyclo-DOPA and betalamic acid synthesis, possibly through physical separation. This chapter examines an aspect of the second point, whether control of betalain biosynthesis occurs due to upregulation of $D O D$ and $C Y P 76 A D 1$ gene expression.

Using beet as a model for investigating betalain biosynthetic regulation is ideal. Swiss chard comes in a variety of colours, the beet genome is currently being sequenced (Lange et al., 2008; McGrath et al., 2004), and there is an Expressed Sequence Transcript (EST) database available for beet ("The Gene Index Databases"). In addition, two of the main biosynthetic genes, DODA1 and CYP76AD1, have been isolated and characterised for beet. A second $D O D$ gene was also identified in beet, $D O D A$ (Christinet et al., 2004). Recombinant DODA can do the 4,5-cleavage of DOPA in vitro (Gandía-Herrero \& García-Carmona, 2012), although it is unclear whether DODA is involved in the betalain pathway. Beet was also produces the following flavonoids: kaempferol (Pyo et al., 2004), flavanones and isoflavones (Kujala et al., 2002; Martin, 1977) in its leaves, and proanthocyanidins in its seeds (Bittrich \& Amaral, 1991), indicating that at least part of the flavonoid pathway is active.

The presence of active flavonoid genes in Swiss chard enables us to test whether betalains and flavonoids share regulatory mechanisms. This can be tested through the induction of betalains, and by monitoring the transcript levels of both betalain and flavonoid biosynthetic genes. The three flavonoid genes chosen to monitor in this study were: chalcone synthase ( $\mathrm{CHS}$ ) and two UDP-glucose:flavonoid-O-glucosyltransferases (UGT71F1 and UGT73A4). CHS was chosen as a marker for up-regulation of the flavonoid biosynthetic pathway as it up-regulates in response to wounding in other plants (Djordjevic et al., 1997; Richard et al., 2000). In addition, other biotic and abiotic factors induce production of flavanones and isoflavones in beet (Martin, 1989; Richardson, 1981), which require CHS for their synthesis. Beet flavonoid glucosyltransferases, UGT71F1 and UGT73A4, have been implicated as possible betanidin glucosyltransferases (Isayenkova et al., 2006). The activities of these two 
enzymes were regioselective to flavonoids in vitro, although, they both had a low affinity for glycosylating betanidin (Isayenkova et al., 2006). UGT71F1 and UGT73A4 were monitored in this study to test if these genes up-regulate in co-ordination with betalain biosynthetic genes.

\subsubsection{Aim}

The aim of this chapter was to compare the regulation of flavonoid and betalain genes in the betalain-producing plant Swiss chard. To test whether betalain and flavonoid biosynthetic genes are co-regulated in Swiss chard, a physical wounding experiment involving RT-qPCR was conducted using Swiss chard leaves. If betalain biosynthetic genes use flavonoid regulatory mechanisms, the assumption was made that these genes would have similar expression patterns in response to wounding. Transient gene expression assays were also used to investigate the functions of the two beet $D O D$ genes as well as examine betalain biosynthetic control in Swiss chard. White-petioled Swiss chard expression patterns were measured as a negative betalain production control.

\subsection{Methods}

\subsubsection{Proanthocyanidin staining in Swiss chard seeds}

Swiss chard seeds were tested for the presence of proanthocyanidins following methods by Li et al. (1996). Swiss chard seed capsules were lightly crushed and added to $0.3 \%$ (w/v) DMACA-HCl solution (Appendix 1.7.3). A separate reaction with Arabidopsis thaliana seeds was used as a positive control. After $20 \mathrm{~min}$, seeds were washed with $70 \%(\mathrm{v} / \mathrm{v})$ ethanol till clear. The blue staining of tissues indicated the presence of proanthocyanidins.

\subsubsection{Wounding RT-qPCR experiment}

RT-qPCR was carried out on wounded Swiss chard (Beta vulgaris subsp. cicla cv. 'Bright Lights') leaves to measure the regulatory response of betalain and flavonoid biosynthetic genes. Nine white and nine red-petioled Swiss chard plants were grown from seed as described in Section 2.3.1. When the plants had 4 - 5 fully expanded leaves the experiment began. Ten puncture wounds were made on the adaxial surface of each leaf using a key blade, avoiding the main veins. Tissue was sampled by cutting two 
millimetres around the wounding site using a clean scalpel blade. Two wounded sites per leaf and three leaves per plant were sampled for each time point to avoid leaf age effect. Tissue from three plants was pooled for each biological replicate to reduce genetic variability. All tissue excised was immediately snap frozen in liquid nitrogen. Time $0 \mathrm{~h}$ was collected within two minutes of wounding. Collection was carried out in the same way at $24 \mathrm{~h}, 48 \mathrm{~h}, 72 \mathrm{~h}$ and $7 \mathrm{~d}$. Tissue was stored at $-80^{\circ} \mathrm{C}$ until RNA was extracted.

RNA was extracted from frozen plant tissues and cDNA synthesised following methods in Section 2.6.2. cDNA was diluted 30 fold and frozen in aliquots at $-80{ }^{\circ} \mathrm{C}$ for each RT-qPCR run to prevent multiple freeze-thaw events. DNase treated RNA (-RT) controls with Actin (ACT) primers (Appendix 2) were run alongside the reactions with cDNA, to test for successful removal of contaminating genomic DNA with DNaseI.

Primers were designed and tested using software listed in Section 2.5 unless otherwise specified (sequences listed in Appendix 2 and begin with RTPCR). All primer sets had a $\mathrm{T}_{\mathrm{m}}$ of $60 \pm 1^{\circ} \mathrm{C}$, predicted minimal secondary structure and primer-dimering, and amplified a product between $60-200 \mathrm{bp}$. The primer sets were designed for the following genes sourced from Genbank or the Gene Index ("The Gene Index Databases"): ACT actin (Genbank accession \# HQ656028), CHS chalcone synthase (Gene Index \#TC12297), CYP76AD1 cytochrome P450 (Genbank accession \#HQ656024), DODA 4,5-DOPA extradiol dioxygenase (Genbank accession \#AJ583017), DODA1 4,5-DOPA extradiol dioxygenase (Genbank accession \# HQ656027), EF2 elongation factor 2 (Genbank accession \#Z97178), UGT73A4 UDP-glucose:flavonoid-O-glucosyltransferase (Genbank accession \#AY526080) and UGT71F1 UDP-glucose:flavonoid-O-glucosyltransferase (Genbank accession \#AY526081). Primer sequences for DODA1, CYP76AD1 and ACT were sourced from Hatlestad et al. (2012).

Actin and elongation factor 2 were chosen as housekeeping genes for the normalisation of the RT-qPCR data. The beet actin was successfully used by (Hatlestad et al., 2012) for RT-qPCR, so was selected for use in my study. Elongation factor $1 \alpha$, a common RT-qPCR housekeeping gene was originally tested for use in RT-qPCR. However, suitable primers could not be designed, so EF2 was used instead. 
RT-qPCR was performed using SsoAdvanced Sybr Green Supermix (BioRad) reagents and the Rotor Gene 3000 real-time PCR machine with Rotor Gene 6000 series software 1.7 (Corbett Research). Each cDNA sample was performed with three technical replicates. The RT-qPCR reactions were set up as a master mix for all reactions (excluding template). The reaction master mix together with cDNA or water was dispensed into a 72-well Gene Disk (Corbett Research) with the CAS-1200 liquid handling system (Corbett Robotics). Samples were run with an inter-rotor control on every Gene Disk. The inter-rotor control consisted of $1 \mu \mathrm{L}$ from each cDNA sample within a biological replicate combined and diluted 100 fold. Gene Disks were run 'sample rich', meaning that all time points for both red- and white-petioled samples were on the same Gene Disk, separating samples by gene of interest (GOI). Fluorescence measurements were performed at $72{ }^{\circ} \mathrm{C}$ each cycle and continuously during final melting. The following cycling parameters and reaction volumes were used for RT-qPCR:

Initial denaturation

Denaturation

Annealing

Final melt curve

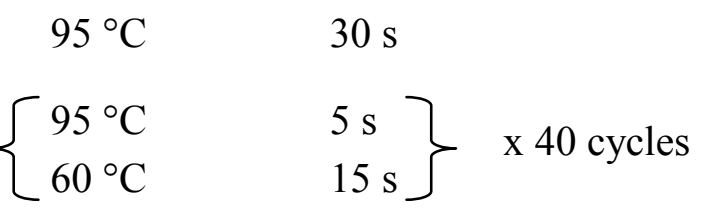

$65-95{ }^{\circ} \mathrm{C} \quad 0.5^{\circ} \mathrm{C}$ increments, 2 s each step

\section{$1.5 \mu \mathrm{L} \quad$ Sterile $\mathrm{H}_{2} \mathrm{O}$}

$5 \mu \mathrm{L} \quad 2 \mathrm{x}$ SsoAdvanced Sybr Green Supermix

$0.5 \mu \mathrm{L} \quad 10 \mu \mathrm{M}$ forward strand primer

$0.5 \mu \mathrm{L} \quad 10 \mu \mathrm{M}$ reverse strand primer

$\frac{2.5 \mu \mathrm{L}}{10 \mu \mathrm{L}}$ Diluted cDNA or water

All RT-qPCR reaction products were cloned (Section 2.7) and sent for sequencing (Section 2.6.9). Each gene had two individual clones sequenced to confirm their identity.

Threshold value $(C t)$ and amplification efficiencies (E) for each quantitative PCR reaction was calculated using the Comparative Quantitation Analysis in the Rotor Gene 6000 software. Results from RT-qPCR was then analysed in two ways, (1) fold change in gene expression relative to time $0 \mathrm{~h}$, and (2) normalised relative quantity (NRQ) for a 
measure of gene transcript levels relative to the housekeeping genes, ACT and EF2. Fold change from $0 \mathrm{~h}$ was calculated by comparing calibrated GOIs to the geometric mean of the housekeeping genes, $A C T$ and EF2, using BestKeeper software (Pfaffl et al., 2004), followed by the Pfaffl equation (Pfaffl, 2001):

$$
\text { Fold change }=\frac{\left(\mathrm{E}_{\mathrm{S}}\right)^{\Delta C t_{\mathrm{SC}}(\text { control-treated })}}{\left(\mathrm{E}_{\mathrm{R}}\right)^{\Delta C t_{\mathrm{RC}}(\text { control-treated })}}
$$

$E_{S}$ and $E_{R}$ are the amplification efficiency values of the sample and reference genes, respectively. $C t_{\mathrm{SC}}$ and $C t_{\mathrm{RC}}$ are the calibrated threshold cycles for the sample and reference genes, respectively. Calibration ensured that inter-rotor variation was minimised. $C t$ values were calibrated using the equations below, where $C t_{\mathrm{i}}$ is the threshold cycle of the inter-rotor control.

$$
\begin{aligned}
& C t_{S C}=C t_{S}-C t_{i} \\
& C t_{R C}=C t_{R}-C t_{i}
\end{aligned}
$$

NRQ was calculated for each GOI to compare the relative gene transcript abundance for red and white Swiss chard at each time point. Relative transcript levels were calculated from the ratio of calibrated GOIs to the geometric mean of housekeeping genes, with the following equation (Rieu \& Powers, 2009):

$$
\mathrm{NRQ}=\frac{R Q_{\mathrm{S}}}{R Q_{\mathrm{R}}}
$$

$R Q_{\mathrm{S}}$ and $R Q_{\mathrm{R}}$ are the relative quantity of the sample and reference genes, respectively. These were calculated with the following equations (where $C t_{\mathrm{C}}$ is the threshold cycle of the calibration control):

$$
\begin{aligned}
& R Q_{S}=\frac{1}{E_{S}{ }^{C t_{S}-C t_{C}}} \\
& R Q_{R}=\frac{1}{E_{S}{ }^{C t_{R}-C t_{C}}}
\end{aligned}
$$


Student $t$-tests were carried out on the RT-qPCR data using Excel. Statistical comparisons were made for fold change from $0 \mathrm{~h}$, and to compare the NRQ values of red and white tissues for each GOI. The significance cut off point was $P$-value $=0.1$.

\subsubsection{Methods for phylogenetic analyses}

\subsubsection{Sequence alignments of $C H S$}

The contig EST of CHS isolated from Beta vulgaris was pieced together by the Gene Index Databases (TC12297; "The Gene Index Databases"). This sequence was aligned against the equivalent regions of the following $C H S$ genes and other structurally similar polyketide synthase genes sourced from GenBank (accession numbers in brackets): CHS: Allium cepa (AAO63021), Anthurium andraeanum (ABE01413), Antirrhinum majus (P06515), Arabidopsis thaliana (NP_196897), Bromheadia finlaysoniana (O23729), Dendrobium hybrid (AAU93767), Iris x hollandica (BAF99252), Oncidium hibrid (ABS58499), Phalaenopsis hyprid (AAY83389), Secale cereale (P53414), Vitis vinifera (BAB84112), Zea mays (P24824); StCS1 (stilbenecarboxylate synthase 1): Marchantia polymorpha (AAW30009). BBS (bibenzyl synthase): Bromheadia finlaysoniana (CAA10514), Phalaenopsis sp. (P53416); STS (stilbene synthase): Vitis vinifera (ABD64685).

The amino acid sequences were aligned using ClustalW followed by maximum likelihood (ML) analysis using the software, MEGA 5.2 (Tamura et al., 2011). Analysis was run using the substitution model Jones-Taylor-Thornton (JTT) with default parameters, ML heuristic method set to nearest neighbour interchange (NNI) and with the branch swap filter set to very strong. Bootstrap analysis with 1000 replicates was used to estimate the confidence of each tree node.

\subsubsection{Sequence alignment of LigB homologues to compare $D O D A$ and $D O D A 1$}

The LigB homologues amino acid sequences were aligned using ClustalW. A phylogenetic tree was then produced using the PhyML programme (Guindon \& Gascuel, 2003) within the bioinformatics software Geneious (version 6.1.6; Biomatters, Auckland, New Zealand). The following default parameters were used for the LG substitution model: proportion of variable sites set to fixed 0 ; number of substitution rate categories set to 4; and gamma distribution parameter set to estimate. The tree was set to optimising topology/length/rate, and the topology search set to NNI (Default, 
fast). Bootstrap analysis with 1000 replicates was used to estimate the confidence of each tree node. LigB homologue from Arabidopsis was used as the root.

The following sequences, sourced from GenBank, were aligned (with accession numbers in brackets): Arabidopsis thaliana AtLigB (NP567456), Populus trichocarpa $\mathrm{x}$ Populus deltoids PxLigB (DT510620), Papaver bracteatum PbLigB (JG633131), Dianthus superbus DsLigB (JL390264), Parakeelya PspLigBI, Salicornia europaea SeLigBI (GAIA01075260), Beta vulgaris BvDODA1 (HQ656027), Amaranthus hypochondriacus AhDODA (HQ889614), Beta vulgaris BvDODA (AJ583017), Ptilotus PhybDOD-like (KF747354), Chenopodium quinoa CqLigBII (CN781996), Salicornia europaea SeLigBII (GAIA01011644), Suaeda salsa SsDODA (ACO59903), Phytolacca americana PaDOD1 (BAH66635), Phytolacca americana PaDOD2 (BAH66636), Mesembryanthemem crystallinum McDOD2 (BE131205), Parakeelya PspDOD-like (KF747353), Portulaca grandiflora PgDODA (AJ580598), Parakeelya PspDOD (KF747352), Mesembryanthemem crystallinum McDOD1 (BF480453), Bougainvillea glabra BgDOD (BAG80687), and Mirabilis jalapa MjDOD (BAG80686). The sequence for Silene vulgaris SvLigB was sourced from The Silene vulgaris Transcriptome (isotig 21529 (Sloan et al., 2012)).

\subsubsection{Transient gene expression analyses}

Transient gene expression experiments were carried out on the nivea Antirrhinum majus line (chs mutant AG110) and Swiss chard (Beta vulgaris subsp. cicla cv. 'Bright Lights') tissues to test the function of betalain biosynthetic enzymes, DODA and DODA1. The flowers on Antirrhinum are white because no flavonoids are produced. Tissues used for these transformations were dorsal petal lobes from Antirrhinum (Figure 6.2), harvested from fully expanded flowers with undehised anthers, and the petioles of yellow and white Swiss chard seedlings (Figure 6.3). All tissue was surface sterilised (Section 2.3.2) before transformation. Antirrhinum tissue was shot on the adaxial side of the petal, Swiss chard tissue on the abaxial side. Three biological replicates were carried out for each treatment.

The constructs used for these experiments were all pART7-based plasmids (Gleave, 1992) containing various genes under the control of a CaMV35S promoter and with a OCS terminator. The constructs contained cDNAs for the following genes, DODA1 (pHMN16), DODA (pHMN22), green fluorescent protein (GFP, pRt99-GFP), and 
CYP76AD1 (pKEC1, Appendix 4A). Constructs with DODA1 and DODA were made by Hanh Ngo of Plant \& Food Research, Palmerston North. Constructs were precipitated onto 1 micron gold prior to biolistic shooting. $5 \mu \mathrm{g}$ of plasmid $(2 \mu \mathrm{g}$ for the GFP-containing vector) along with Milli-Q water, to make the volume up to $10 \mu \mathrm{L}$, was pipetted into the gold. Tube was flicked and placed in a vortexing shaker on low. While vortexing, $20 \mu \mathrm{L} 100 \mathrm{mM}$ spermadine and $50 \mu \mathrm{L} 2.5 \mathrm{M} \mathrm{CaCl}_{2}$ were added to the tube. Tube was vortexed on high for $3 \mathrm{~min}$, centrifuged briefly to pellet the gold and $90 \mu \mathrm{L}$ of liquid was discarded. Gold was resuspended before removal of each aliquot used in shooting.

For the shooting, tissue was placed on media \#2 (Appendix 1.1.3) in culture tubs to soften the impact of the gold bombardment. Tissue was shot twice with $5 \mu \mathrm{L}$ of prepared gold suspension using $400 \mathrm{pK}$ helium pressure through a filter cartridge in a vacuum chamber at $-90 \mathrm{kPa}$. Tissue was then placed on fresh media in a temperature controlled room with $16 / 8 \mathrm{~h}$ light/dark cycle overnight.

For vacuum infiltration, $24 \mathrm{~h}$ after shooting tissue was placed in beaker containing $20 \mathrm{~mL}$ Milli-Q water or $10 \mathrm{mM}$ L-DOPA solution (Appendix 1.7.1). Vacuum of $-90 \mathrm{kPa}$ was maintained for $30 \mathrm{~s}$, tissue rinsed with fresh water and blotted dry before being placed back on the media overnight. 


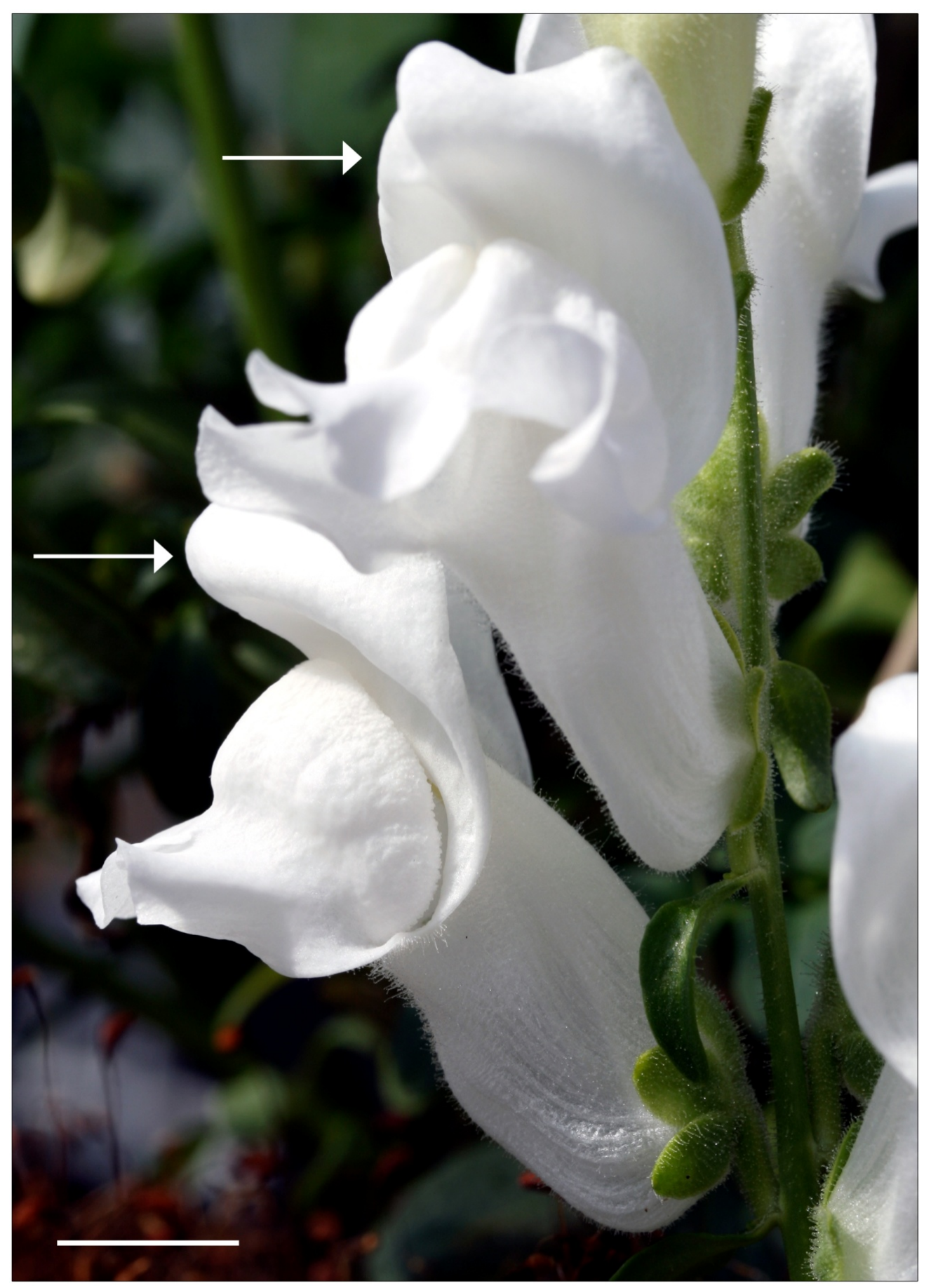

Figure 6.2 Flowers of nivea Antirrhinum majus (chs mutant). Arrows indicate the dorsal petal used in transient expression assays. Scale bar $=10 \mathrm{~mm}$ (approximately). Image supplied by Kevin Davies. 

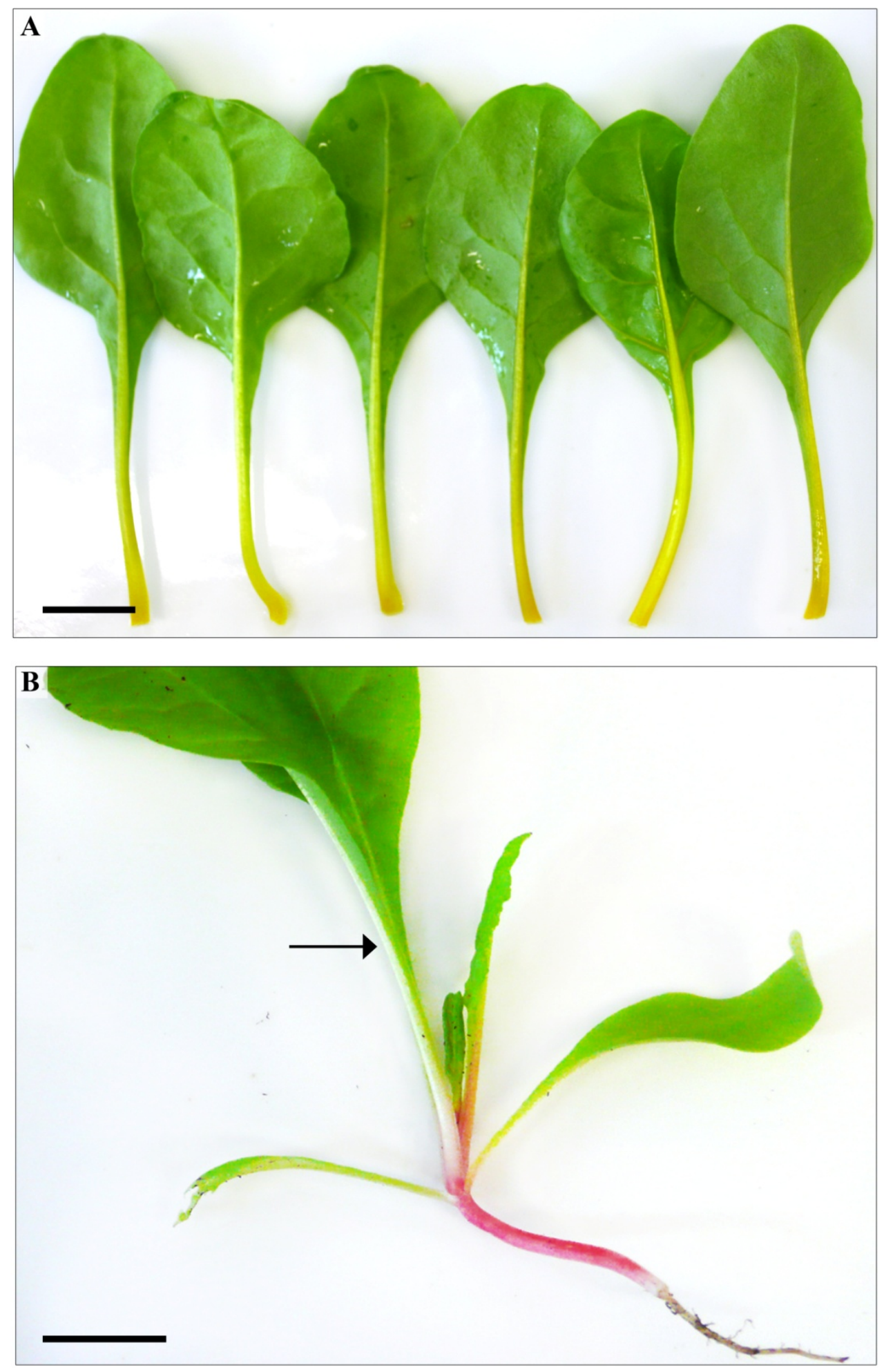

Figure 6.3 Swiss chard tissues used in transient gene expression assays. (A) Yellow petioles; (B) White petioles. Arrow in B indicates an example of petiole used. Scale bars $=10 \mathrm{~mm}$. 


\subsection{Results}

\subsubsection{Swiss chard seeds contained proanthocyanidins}

Beta vulgaris is known to produce various flavonoids in its leaves (Kujala et al., 2002; Martin, 1977; Pyo et al., 2004). This means there are active flavonoid genes in this taxon. Swiss chard seeds were tested for the presence of PAs to determine if the flavonoid pathway was active in this cultivar of beet. Cells of both Arabidopsis and Swiss chard seed testae turned blue to indicate that they both contained proanthocyanidins (Figure 6.4). The presence of proanthocyanins in Swiss chard indicates that the pathway up to DFR was indeed switched on in the seeds, and was functional.

\subsubsection{Phylogenetic analysis of $\mathrm{CHS}$}

Before the gene expression analysis could be done, a $C H S$ sequence from $B$. vulgaris needed to be identified because there were no $C H S$ sequences reported in the literature for this taxon. The putative CHS sequence was found in the Beet index ("The Gene Index Databases") by searching for 'CHS' within the EST annotations. This EST was partial length for the open reading frame, and was tentatively annotated $C H S$ as it had $62 \%$ sequence similarity to a chalcone synthase from Gypsophila paniculata.

Phylogenetic analysis was done to test this annotation.

Phylogenetic analysis of the $C H S$ sequence along with characterised $C H S$ sequences sourced from the NCBI database support this description (Figure 6.5, for alignment see Appendix 6). The beet $C H S$ grouped closely with sequences of confirmed $\mathrm{CHS}$ genes with moderately high bootstrap support. This group of $C H S$ sequences were also in a separate clade from the $B B S, S T S$ and $S t C S 1$ genes. Given the result of this analysis, sequence was assumed to be a $C H S$ and, therefore, included in this study.

\subsubsection{Flavonoid and betalain biosynthetic gene expression after wounding}

Over the seven day wounding experiment, foliar tissue on white-petioled leaves did not change colour, whereas wounded sites on red-petioled leaves developed a red halo (Figure 6.6). The developing halo was first visually detected at $48 \mathrm{~h}$ after wounding and continued to grow and become more deeply coloured through the course of the experiment. Betalain and flavonoid gene expression data was obtained by RT-qPCR. 
The flavonoid genes were $C H S, U G T 73 A 4$ and $U G T 71 F 1$ (Figure 6.7) and the betalain genes were DODA1, DODA and CYP76AD1 (Figure 6.8). Data for each GOI was normalised from the geometric mean of $A C T$ and $E F 2$ housekeeping genes using BestKeeper (Pfaffl et al., 2004). The variation in threshold cycle (Ct) values for $A C T$ and $E F 2$ across the time points was between 18.5 and 22 for $A C T$, and between 19.5 and 22.8 for $E F 2$. Sample variability was minimal in technical replicates, and the inter-rotor controls, which were used as calibration controls, only varied by a maximum $C t$ value of 1 .

In the red line, CHS, UGT73A4 and UGT71F1 were all up-regulated between $24 \mathrm{~h}$ and $48 \mathrm{~h}$. (Figure 6.9). The fold change for CHS was greatest of the three genes, with a $40+$ fold change for each time point. The normalised relative quantity (NRQ) values at each time point mirrored the trends seen in the fold change data (Figure 6.10). Increases in fold change resulted in increased relative transcript levels for all three genes. As betalains clearly accumulated around wounding sites in red-petioled leaves after $48 \mathrm{~h}$ (Figure 6.6), it was expected that the transcript levels of betalain biosynthetic genes, DODA1 and CYP76AD1 which were characterised by Hatlestad et al. (2012), would significantly increase during this period. DODAl, had a trend for up-regulation at $48 \mathrm{~h}$ in response to wounding, however, this was not statistically significant ( $P$-value $=0.11$, Figure 6.11). CYP76ADl also was not up-regulated in the red line $(24 \mathrm{~h}, P$-value $=0.70$, Figure 6.11). In addition, the other $D O D$ gene $D O D A$, which was demonstrated to have $D O D$ activity in vitro (Gandía-Herrero \& García-Carmona, 2012), also had a trend for up-regulation at $24 \mathrm{~h}$ after wounding but this was not statistically significant $(P$-value $=$ 0.13, Figure 6.11). Unlike what was observed for the flavonoid genes, the NRQs of $D O D A 1$ and $C Y P 76 A D 1$ did not follow the same trends as the fold change graphs (Figure 6.12). NRQ data revealed high relative transcript levels for DODA1 and CYP76AD1 at $0 \mathrm{~h}$, indicating that these genes were already highly expressed at the time of wounding. In addition, the fold change measured for DODA1 at $48 \mathrm{~h}$ (Figure 6.11) only related to minor increases in relative transcript abundance (Figure 6.12). In contrast, the NRQ trends for DODA (Figure 6.12) were similar to the fold change data (Figure 6.11). This suggests that $D O D A$ expression was not regulated in the same way as DODA1 and CYP76AD1. 
Expression of flavonoid and betalain biosynthetic genes was also monitored in the white line, which was used as a negative betalain biosynthesis control. The regulation patterns of the three flavonoid genes were similar to the red tissues, with the exception that $C H S$ expression in the white line was a quarter to a third of that measured in the red line (Figure 6.8). The NRQ patterns for these genes were also similar to the red-petioled leaf tissues (Figure 6.9). However, the relative abundance of $C H S$ was higher in the white than the red line at all time points, which would explain why the fold change was lower in the white line. The expression of betalain biosynthetic genes in the white-petioled leaf tissues differed from that in the red tissues. Similarly to what was found in the red line, $D O D A 1$ was not significantly up-regulated in the white line $(P$-value $=0.39$, Figure 6.11). However, both $D O D A$ and $C Y P 76 A D 1$ were up-regulated in the white tissues after $24 \mathrm{~h}(D O D A P$-value $=0.07, C Y P 76 A D 1 P$-value $=0.01$, Figure 6.11 $)$. Despite the significant fold change in these two genes, the changes in relative abundance were minor, especially for CYP76AD1 (Figure 6.12).

\subsubsection{Phylogenetic analyses of $D O D A$ and $D O D A 1$}

Phylogentic analyses of $D O D A$ and $D O D A 1$ were carried out to compare the similarities of these genes with other characterised $D O D$ genes in the betalain taxa. $D O D$ sequences from different betalain taxa representatives were sourced from the NCBI databases (for details on alignment, see Appendix 7). Beet DODA1 and DODA formed different subclades within the Amaranthaceae clade (Figure 6.13). The position of BvDODA1 on the outside of the Amaranthaceae clade suggests that it this $D O D$ gene differs from the others in Amaranthaceae. Therefore, the positioning on the tree and sequence differences between $D O D A 1$ and $D O D A$ may be due to different functional roles.

\subsubsection{Transient betalain biosynthetic gene expression in Antirrhinum}

Following the methods used in Harris et al. (2012), betalain biosynthetic genes were transiently expressed in A. majus (nivea) petal tissue. The nivea line of A. majus was used for these experiments as it is a chs mutant and, therefore, is unable to produce anthocyanins. Also, this plant does not naturally produce betalains. The transient expression was carried out to test whether the two beet $D O D$ genes identified to have DOD activity and thus may be involved in betalain biosynthesis. Transformed tissue was vacuum infiltrated $24 \mathrm{~h}$ after the biolistic bombardment with the betalain precursor L-DOPA or water as a control. In addition, expression of the green fluorescence protein 
$(G F P)$ was used as a visual marker for transformation efficiency. GFP autofluoresces green under blue light, making it easy to detect in plant tissue.

As expected for the transformed tissues vacuum infiltrated with water, there was no colour change under white light (Figure 6.14). Similarly, all treatments resulted in no autofluorescence under blue light except for the transformation control, $D O D A+G F P$ (Figure 6.14I). Tissue shot with DODA + GFP had a large number of blue/green autofluorescent cells under blue light, which indicated good transformation efficiency. Tissue vacuum infiltrated with L-DOPA and shot with the same constructs had different results. Tissue expressing $D O D A 1$ produced yellow and pink cells with strong green autofluorescence under blue light, indicating the presence of betaxanthins (Figure 6.14B). The tissue expressing constructs $D O D A 1+C Y P 76 A D 1$ had a greater number of red, orange and pink cells than tissue with DODAl alone, with a decreased number of yellow cells, indicating the production of both betaxanthins and betacyanins (Figure 6.14D). The autofluorescence of this tissue was also weaker than with $D O D A 1$ alone tissue, which was expected with co-localisation of betaxanthins and betacyanins, as betacyanins obscure betaxanthin autofluorescence (see Figure 5.6). DODA and DODA+ CYP76AD1 shot tissue had no colour change under white light, but had a few weakly green autofluorescent cells under blue light (Figure 6.14F and 6.14H). Given that there was no visible colour change, this fluorescent compound may not be a betalain. Alternatively, betaxanthins were produced but at a level too low to see under white light. L-DOPA fed DODA + GFP tissue had a similar number of autofluorescent cells to water fed $D O D A+G F P$ tissue (Figure 6.14J), which indicates similar transformation efficiency between treatments. The CYP76AD1 only tissue had no changes in colour or autofluorescence (Figure 6.14L).

These results indicated that betaxanthins were likely only produced from transient expression of $D O D A 1$ and not $D O D A$, when L-DOPA was present. The co-expression of $D O D A 1+C Y P 76 A D 1$ also appeared to induce the production of both betaxanthins and betacyanins, which again was not observed in the tissues transformed with DODA+ CYP76AD1. The lack of pigments in tissues transformed with $D O D A$ is unlikely to be due to a faulty construct. The $D O D A$ sequence in construct pHMN22 was commercially synthesised so contained no mutations. Therefore, these results indicate that only $D O D A 1$ has a role in betalain production. 


\subsubsection{Transient betalain biosynthetic gene expression in Swiss chard}

Transient betalain biosynthetic gene expression assays were carried out using Swiss chard petioles to further investigate biosynthetic regulation of betacyanins and betaxanthins in yellow and white lines. Betacyanin production in yellow Swiss chard petioles was complemented with the expression of CYP76AD1. Transient expression of CYP76AD1 in yellow tissues turned cells red (Figure 6.15). This colour change was not a stress-induced response, as there was no colour change for tissue shot with the GFP construct only.

In the white line, induction of betalain production was attempted by feeding L-DOPA solution through base of petioles. However, L-DOPA just oxidised around the base of the petioles and no betalains were visibly produced (data not shown). Betalain production in white Swiss chard petioles was also not complemented by the transient expression of either DODA1 or DODA. Transformation of these tissues resulted in no changes to colour or autofluorescence (Figure 6.16A and 6.16C). There was no visible colour change in white light when the shot tissue was also fed L-DOPA (Figure 6.16B and 6.16D). However, under blue light $D O D A 1$ shot tissue had a number of green autofluorescent cells and $D O D A$ tissue a few foci of weak autofluorescence, similar to the autofluorescence seen in Antirrhinum tissue. The transformation efficiency control, $D O D A+G F P$, had a number of blue/green cells for both treatments (Figure 6.16E and 6.16F). This indicated that the weak production of betaxanthins was not due to poor transformation rates. The fast oxidation of L-DOPA could possibly have caused reduced levels of betaxanthins being produced in DODA1 shot tissues. It was observed that within minutes of vacuum infiltration with L-DOPA, the tissues quickly oxidised this compound despite the immediate rinsing of tissues with water (data not shown). 

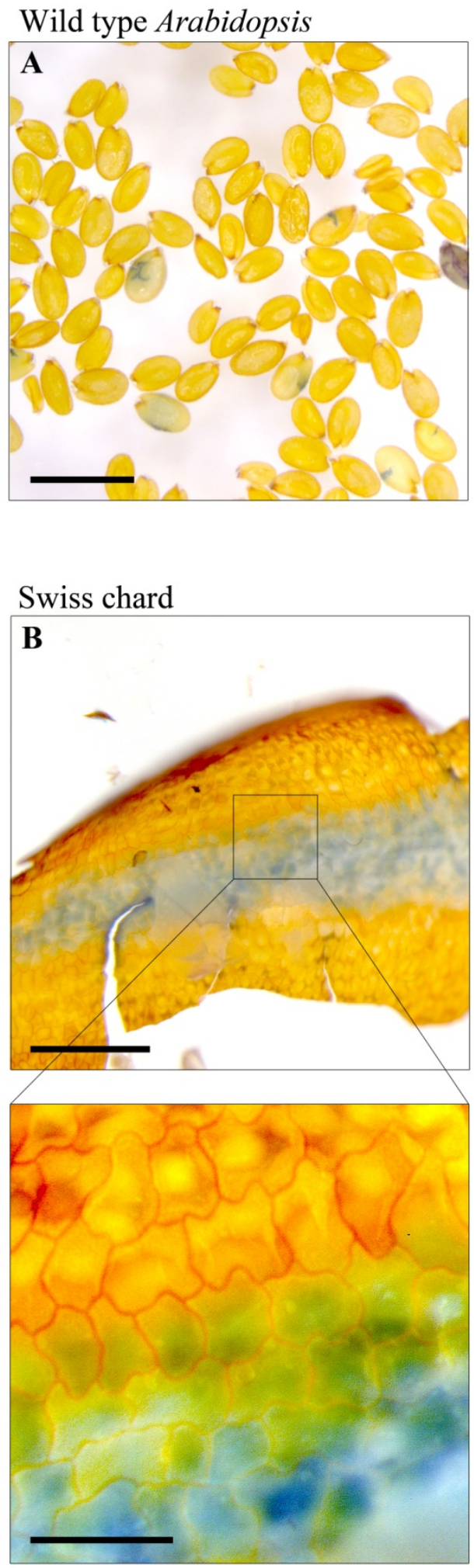

Figure 6.4 Seeds of (A) wild type Columbia Arabidopsis thaliana and (B) Swiss chard with $0.3 \% \quad(\mathrm{w} / \mathrm{v})$ DMACA-HCl staining (blue) indicating the presence of proanthocyanidins. Scale bars: $\mathbf{A}=1 \mathrm{~mm}, \mathbf{B}=400 \mu \mathrm{m}$ and insert $=50 \mu \mathrm{m}$ 


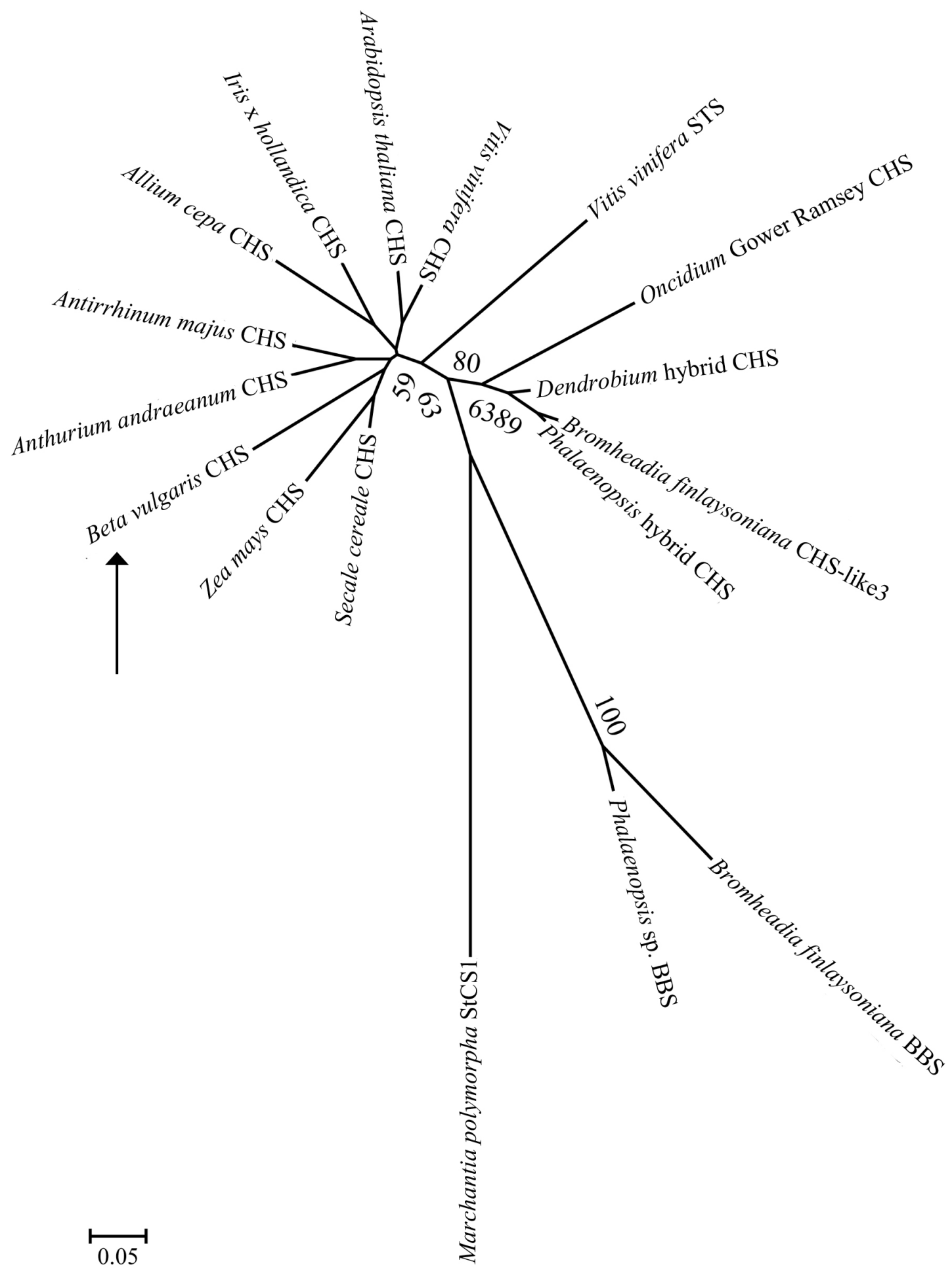

Figure 6.5 Unrooted phylogenetic analysis of a putative Beta vulgaris CHS. Sequences used include $C H S$ and other polyketide synthases (STS stilbene synthase; BBS bibenzyl synthase). For Genbank accession number see Section 6.2.3.1. Bootstrap analysis used 1000 datasets and results are shown for nodes that have at least $50 \%$ support. The arrow indicates the $B$. vulgaris $C H S$ sequence analysed in this study. The branch-lengths indicate the average number of amino acids substitutions per site, with the scale given on the scale bar at the bottom of the figure. See Appendix 6 for the sequence alignment used in the analysis. 
White-petioled leaves
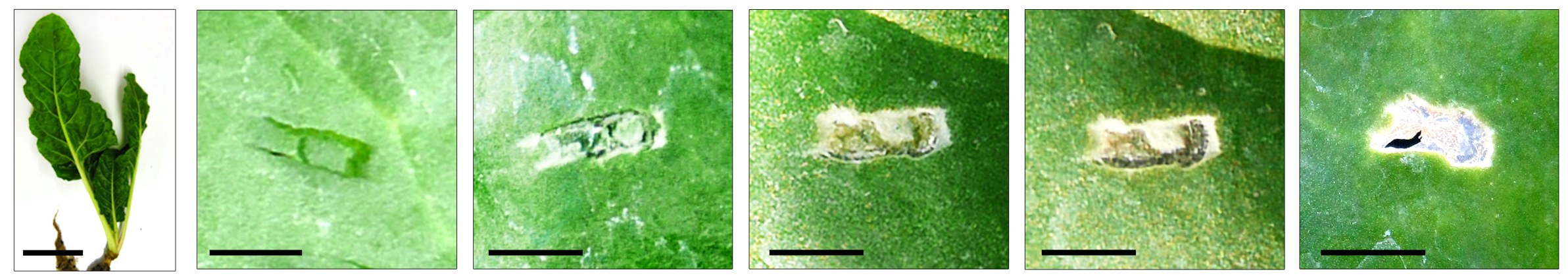

Red-petioled leaves

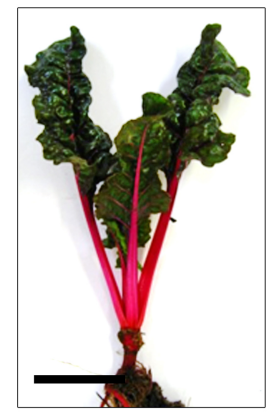

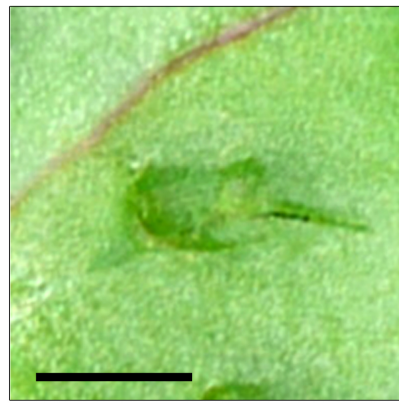

$0 \mathrm{~h}$

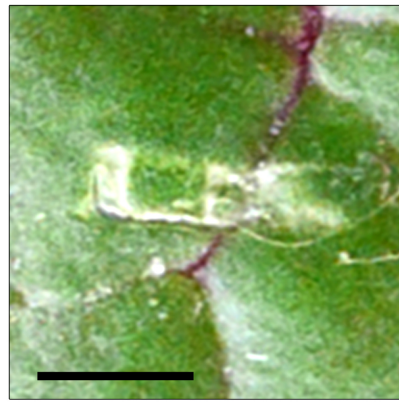

$24 \mathrm{~h}$

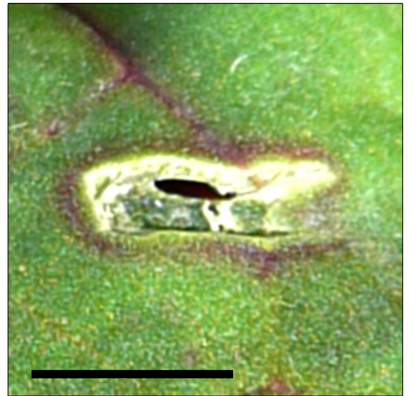

$48 \mathrm{~h}$

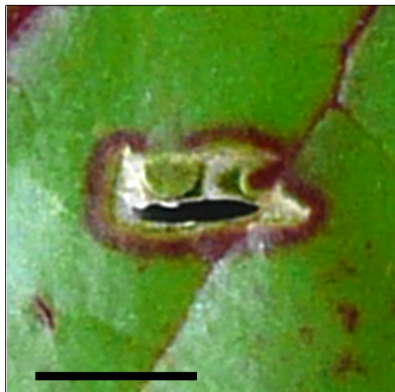

$72 \mathrm{~h}$

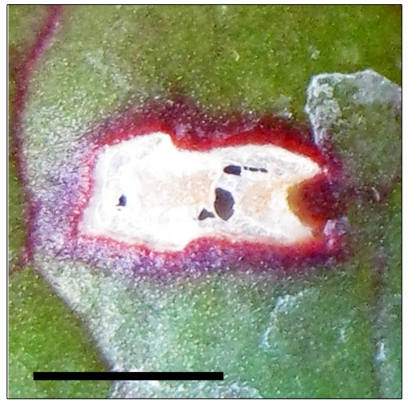

$7 \mathrm{~d}$

Figure 6.6 Pigment accumulation patterns in wounded red- and white-petioled Swiss chard leaves over $7 \mathrm{~d}$ for RT-qPCR experiment. Scale bars: whole plants $=50 \mathrm{~mm}$, leaf close ups $=5 \mathrm{~mm}$. 


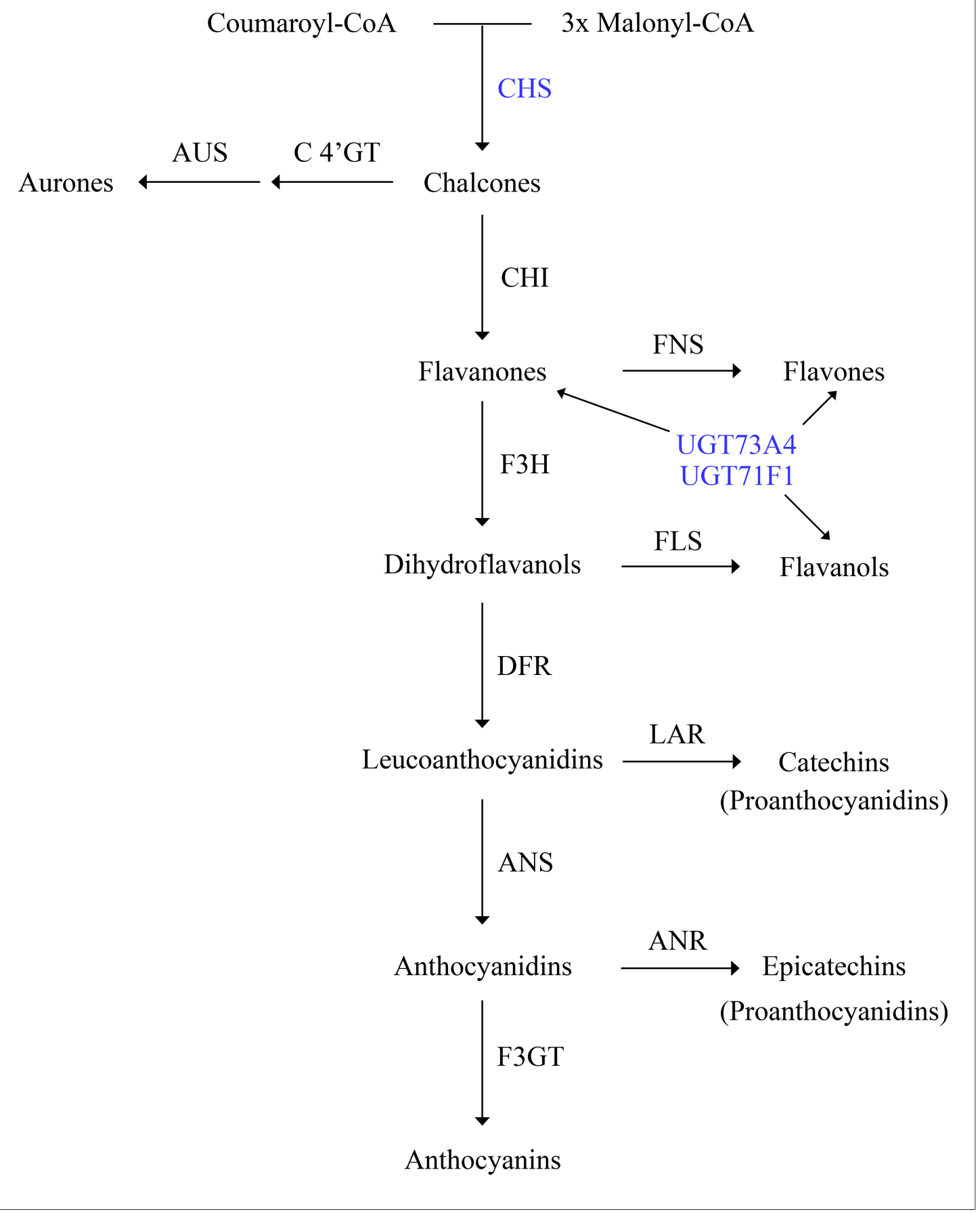

Figure 6.7 The flavonoid biosynthetic pathway. Enzyme code (gene transcript monitored with RT-qPCR in blue): ANR anthocyanidin reductase, ANS anthocyanidin synthase, AUS aureusidin synthase, C 4'GT chalcone 4'-O-glucosyltransferase, CHI chalcone isomerase, CHS chalcone synthase, DFR dihydroflavonol reductase, F3GT UDP-glucose:flavonoid 3-O-glucosyltransferase, F3H flavanone-3-hydroxylase, FLS flavonol synthase, FNS flavone synthase, LAR leucoanthocyanidin reductase, UGT73A4/UGT71F1 UDP-glucose: flavonoid 3-O-glucosyltransferase (glycosylates flavanones, flavones and flavanols). 


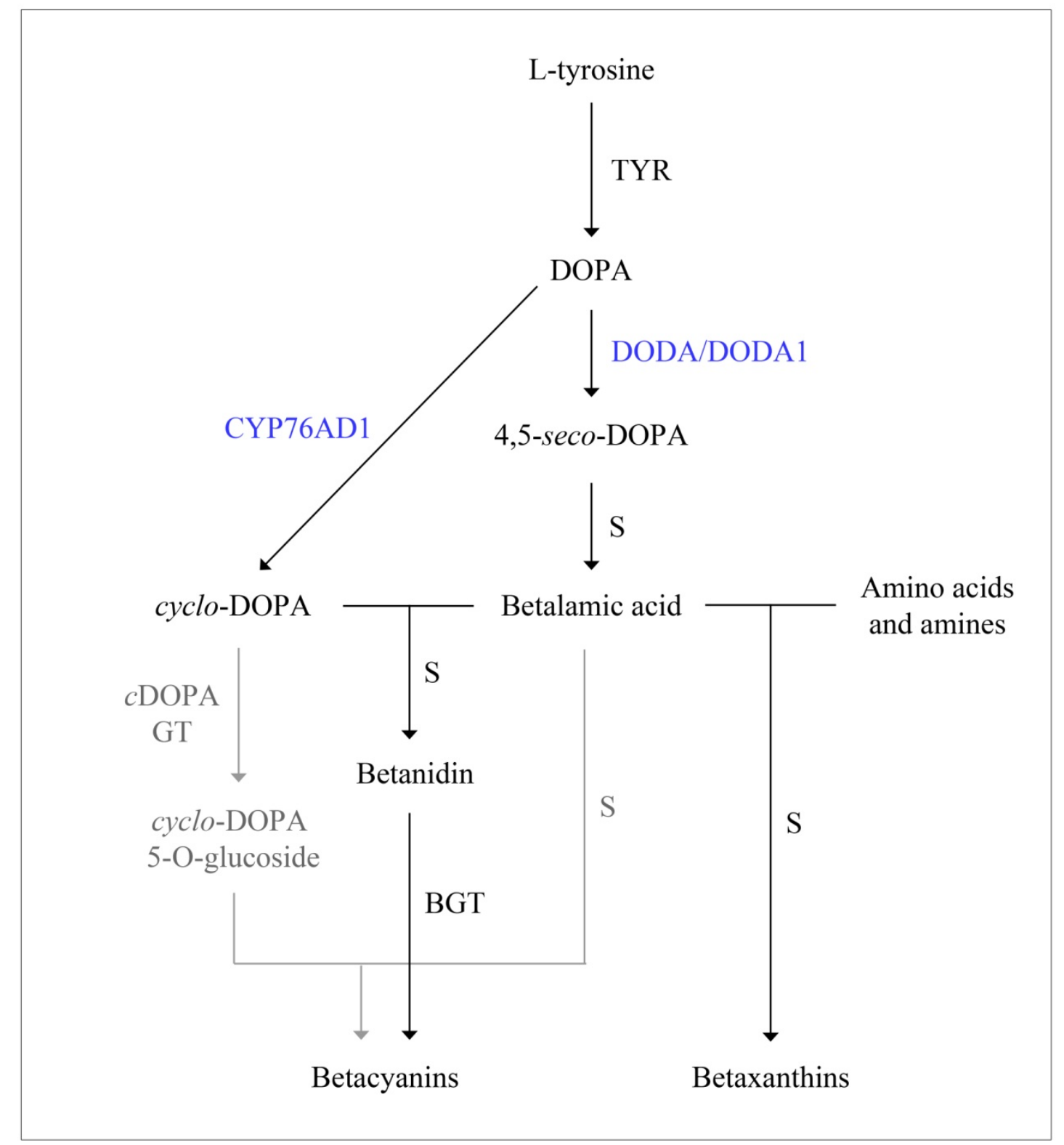

Figure 6.8 The betalain biosynthetic pathway. Enzyme code (gene transcript monitored with RT-qPCR in blue): BGT betanin glycosyltransferase cDOPA GT cyclo-DOPA glycosyltransferase, CYP76AD1 cytochrome P450, DODA/DODA1 4,5-DOPA extradiol dioxygenase, TYR tyrosinase. Grey indicates possible alternative glycosylation pathway. Steps labelled $\mathrm{S}$ are spontaneous reactions. 

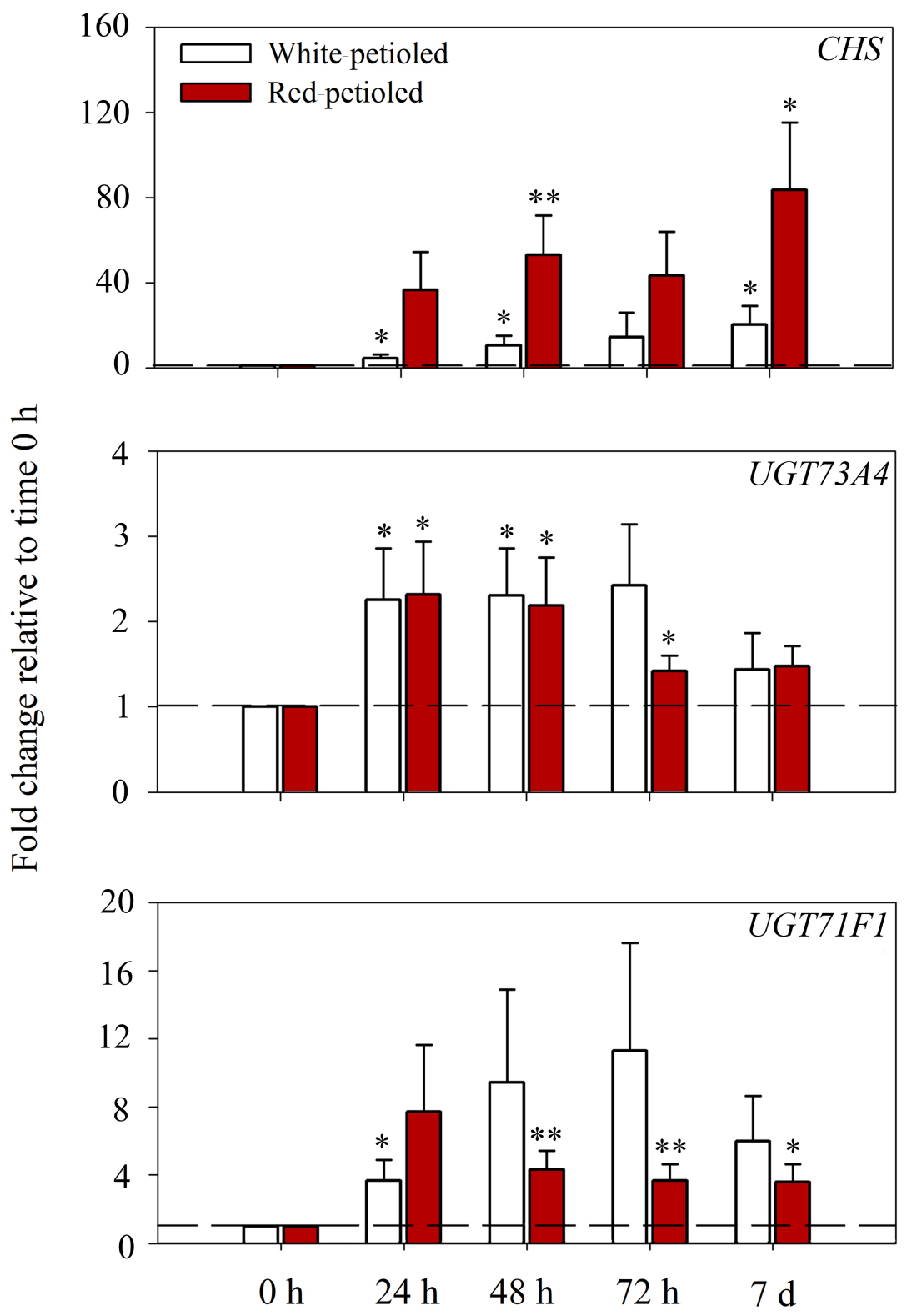

Time Sampled

Figure 6.9 Relative fold change (RT-qPCR) of flavonoid biosynthetic genes from $0 \mathrm{~h}$ (indicated by dashed line) in wounded red- and white-petioled Swiss chard leaves. $C H S$ chalcone synthase, UGT73A4/UGT71F1 UDP-glucose: flavonoid-Oglucosyltransferase. Gene transcripts measured by RT-qPCR, calibrated and normalised to the geometric mean of the housekeeping genes, $A C T$ and EF2. Means \pm S.E., $n=3$. Statistical significance of fold change from $0 \mathrm{~h}$ : ${ }^{*} P$-value $<0.1,{ }^{* *} P$-value $<0.05$, $* * *$ $P$-value $<0.01$. 

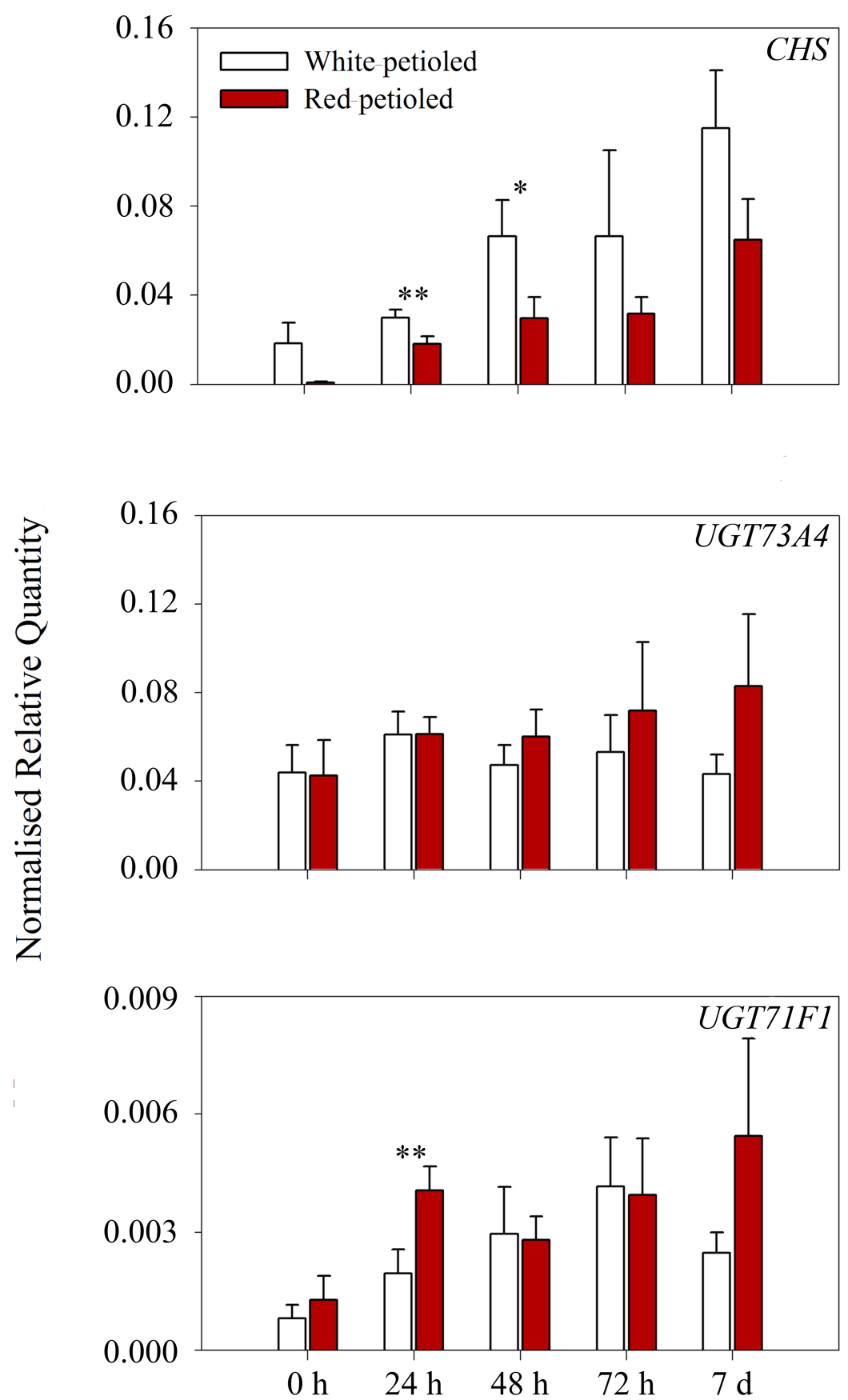

Time Sampled

Figure 6.10 Normalised relative quantity (NRQ) of flavonoid biosynthetic genes after wounding treatment on red- and white-petioled Swiss chard leaves. CHS chalcone synthase, UGT73A4/UGT71F1 UDP-glucose: flavonoid-O-glucosyltransferase. Gene transcripts measured by RT-qPCR, calibrated and normalised to the geometric mean of the housekeeping genes, $A C T$ and $E F 2$. Means \pm S.E., $n=3$. Statistical significance of red- versus white-petioled tissue: $* P$-value $<0.1, * * P$-value $<0.05, * * * P$-value $<$ 0.01 . 

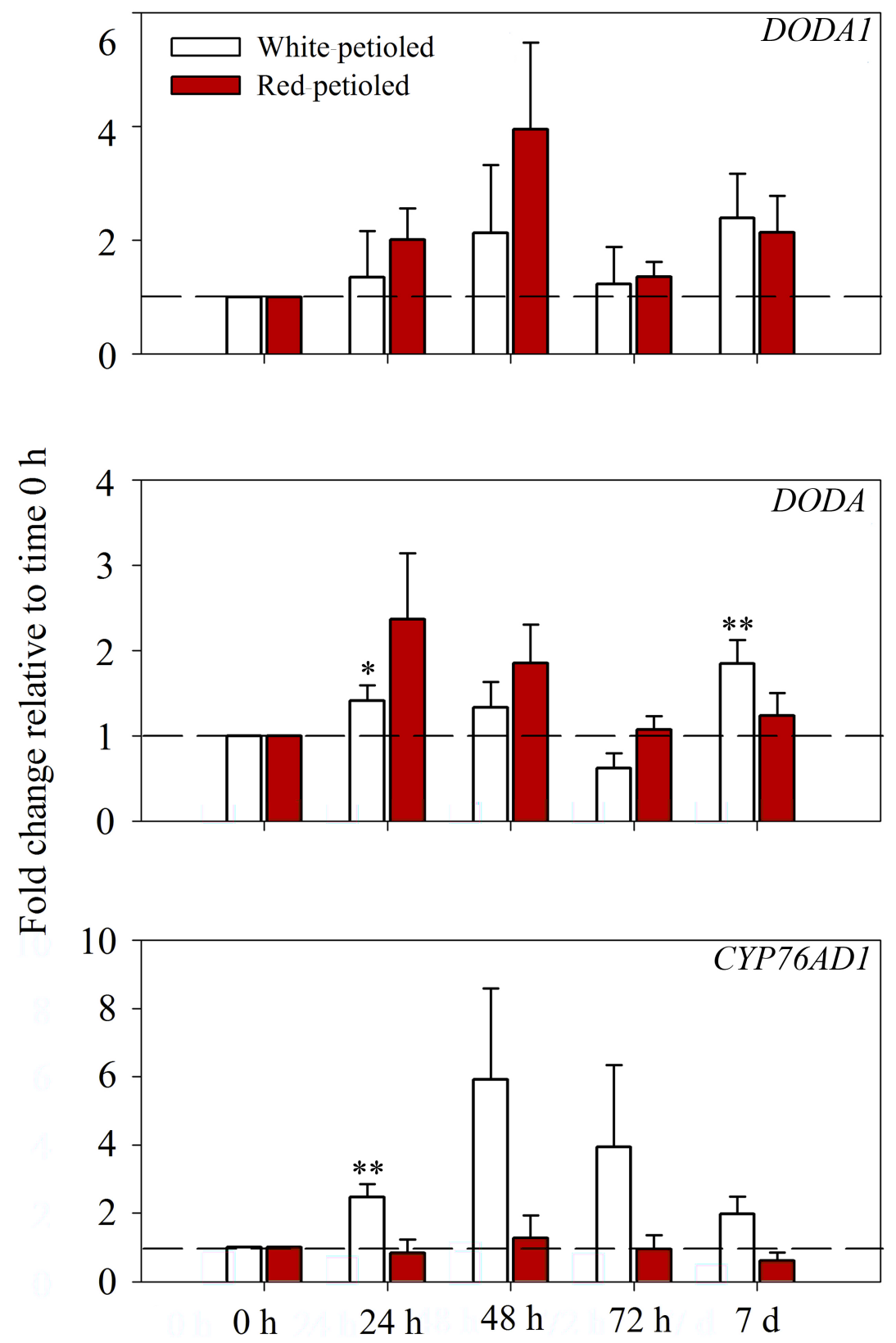

Time Sampled

Figure 6.11 Relative fold change (RT-qPCR) of betalain biosynthetic genes from $0 \mathrm{~h}$ (indicated by dashed line) in wounded red- and white-petioled Swiss chard leaves. CYP76AD1 cytochrome P450, DODA 4,5-DOPA extradiol dioxygenase, DODA1 4,5-DOPA extradiol dioxygenase. Gene transcripts measured by RT-qPCR, calibrated and normalised to the geometric mean of the housekeeping genes, $A C T$ and EF2. Means \pm S.E., $n=3$. Statistical significance of fold change from $0 \mathrm{~h}$ : $* P$-value $<0.1$, ** $P$-value $<0.05, * * * P$-value $<0.01$. 

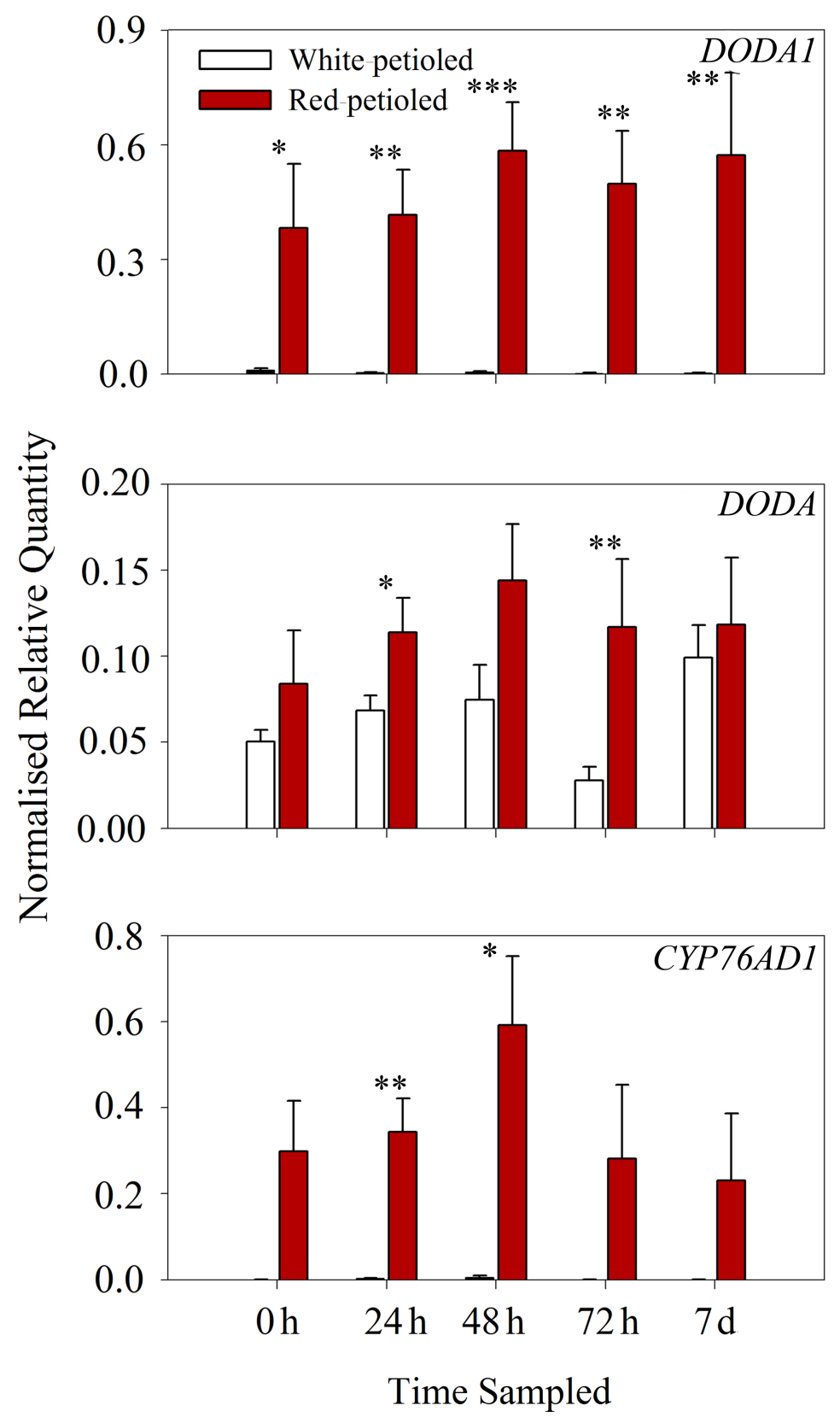

Figure 6.12 Normalised relative quantity (NRQ) of betalain biosynthetic genes after wounding treatment on red- and white-petioled Swiss chard leaves. CYP76AD1 cytochrome P450, DODA 4,5-DOPA extradiol dioxygenase, DODA1 4,5-DOPA extradiol dioxygenase. Gene transcripts measured by RT-qPCR, calibrated and normalised to the geometric mean of the housekeeping genes, $A C T$ and EF2. Means \pm S.E., $n=3$. Statistical significance of red- versus white-petioled tissue: $* P$-value $<0.1$, $* * P$-value $<0.05, * * * P$-value $<0.01$. 


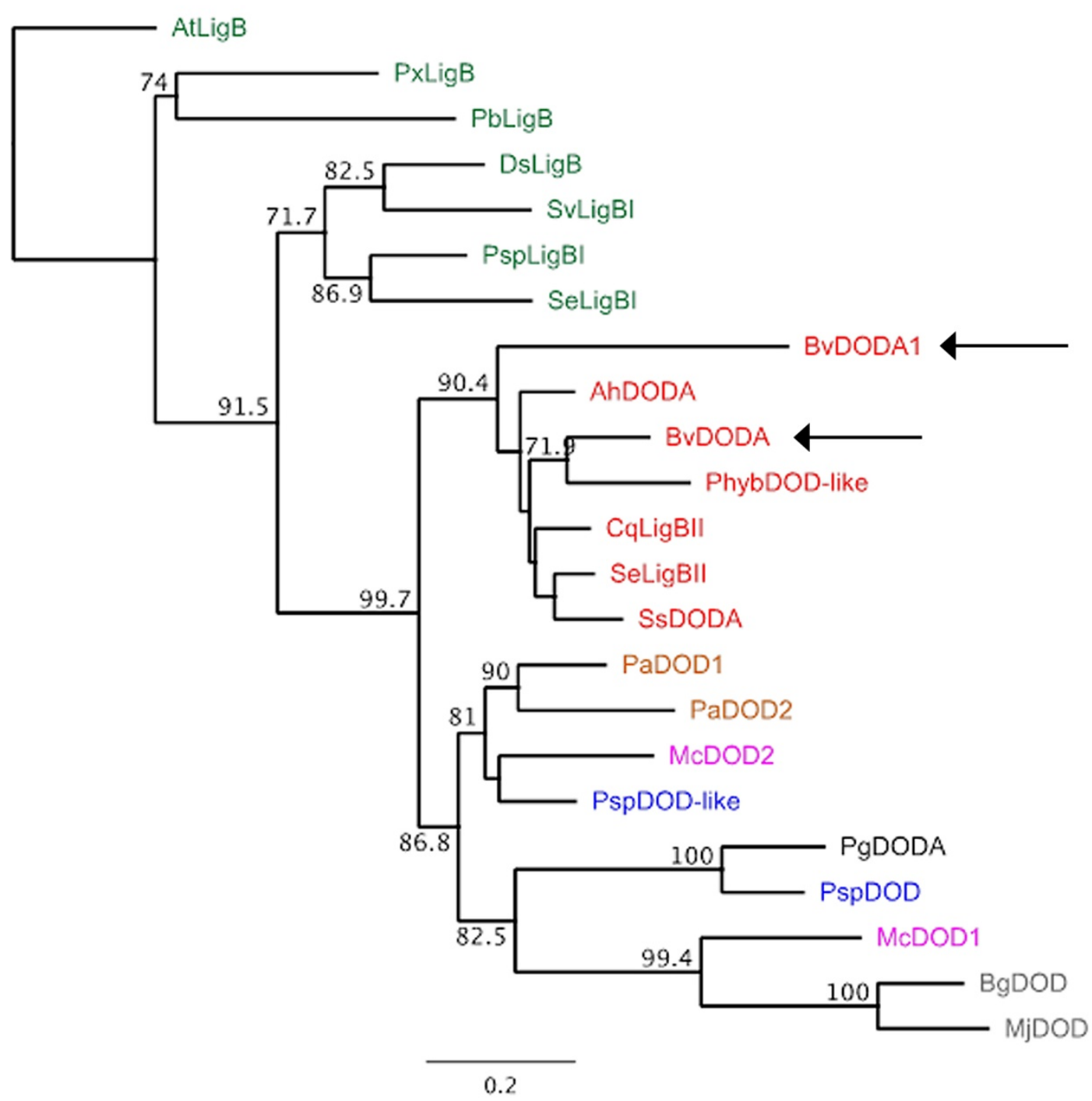

Figure 6.13 Phylogenetic analysis (Maximum Likelihood) comparing beet DODA and $D O D A 1$ with $D O D$ genes from other betalain-producing taxa. $\operatorname{Lig} B$ genes were used as an out-group. Sequences for $M c D O D 1$ and $M c D O D 2$, were not full-length, but spanned the catalytic domain. Bootstrap analysis used 1000 datasets and results are shown for nodes that have at least $70 \%$ support. See Section 6.2.3.2 for information on gene names, species name and accession numbers. Class I genes (green); Class II genes: Amaranthaceae (red); Phytolaccaceae (orange); Aizoaceae (pink); Montiaceae (blue); Portulacaceae (black); Nyctaginaceae (grey). The branch-lengths indicate the average number of amino acids substitutions per site, with the scale given on the scale bar at the bottom of the figure. Arrows indicate Beta vulgaris DOD genes further analysed in this study. See Appendix 7 for the sequence alignment used in the analysis. Phylogenetic alignment and tree supplied by Kathy Schwinn. 


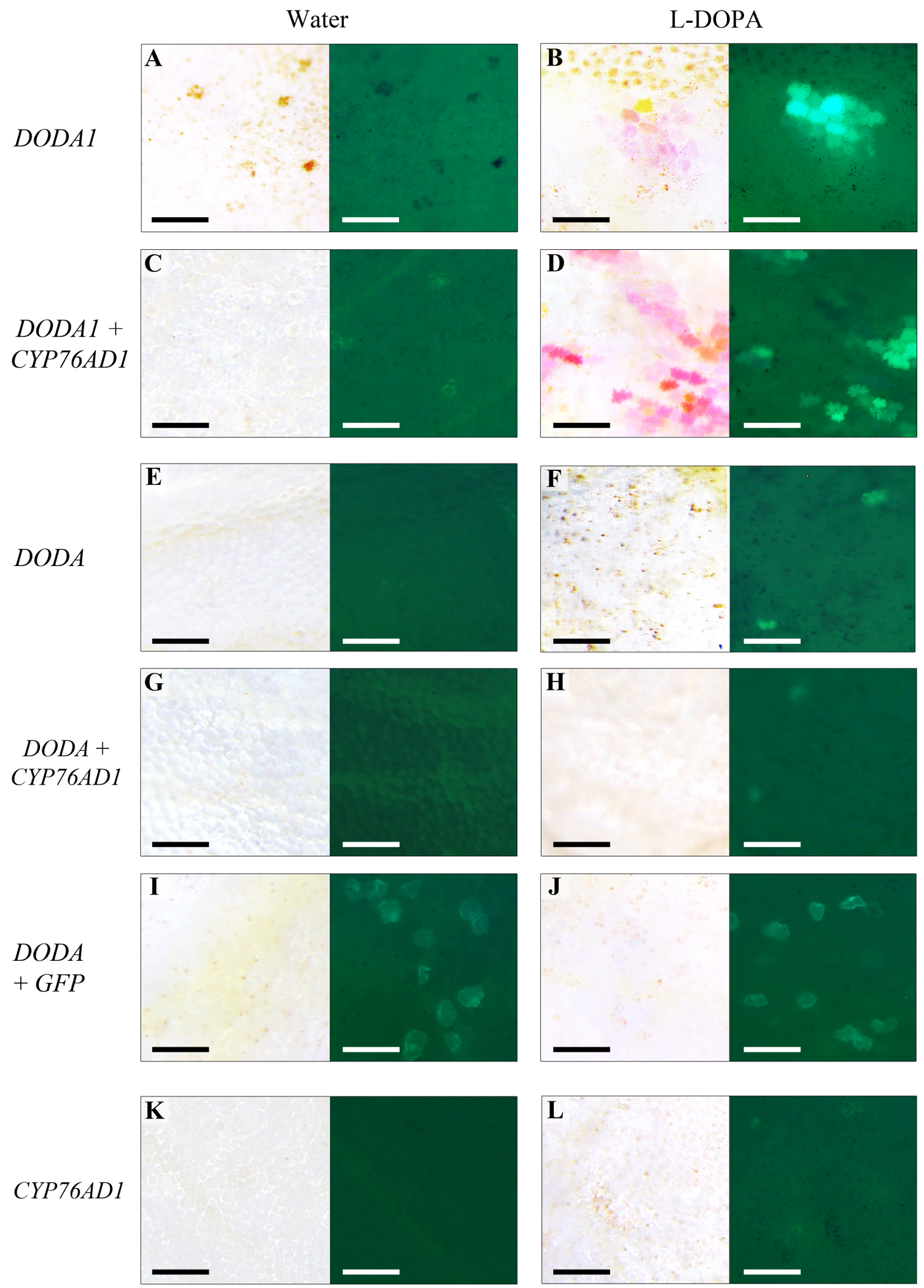

Figure 6.14 Transient betalain gene expression on Antirrhinum majus dorsal petals vacuum infiltrated with water (left) and $10 \mathrm{mM} \mathrm{L-DOPA} \mathrm{(right).} \mathrm{Constructs} \mathrm{examined}$ under white and blue light: (A and B) DODA1; (C and D) DODA1 + CYP76AD1; (E and F) DOD2; (G and H) DODA + CYP76AD1; (I and J) DODA + GFP; (K and L) $C Y P 76 A D 1$. Figure shows representative results for each construct from three replicates. Scale bars $=150 \mu \mathrm{m}$. 

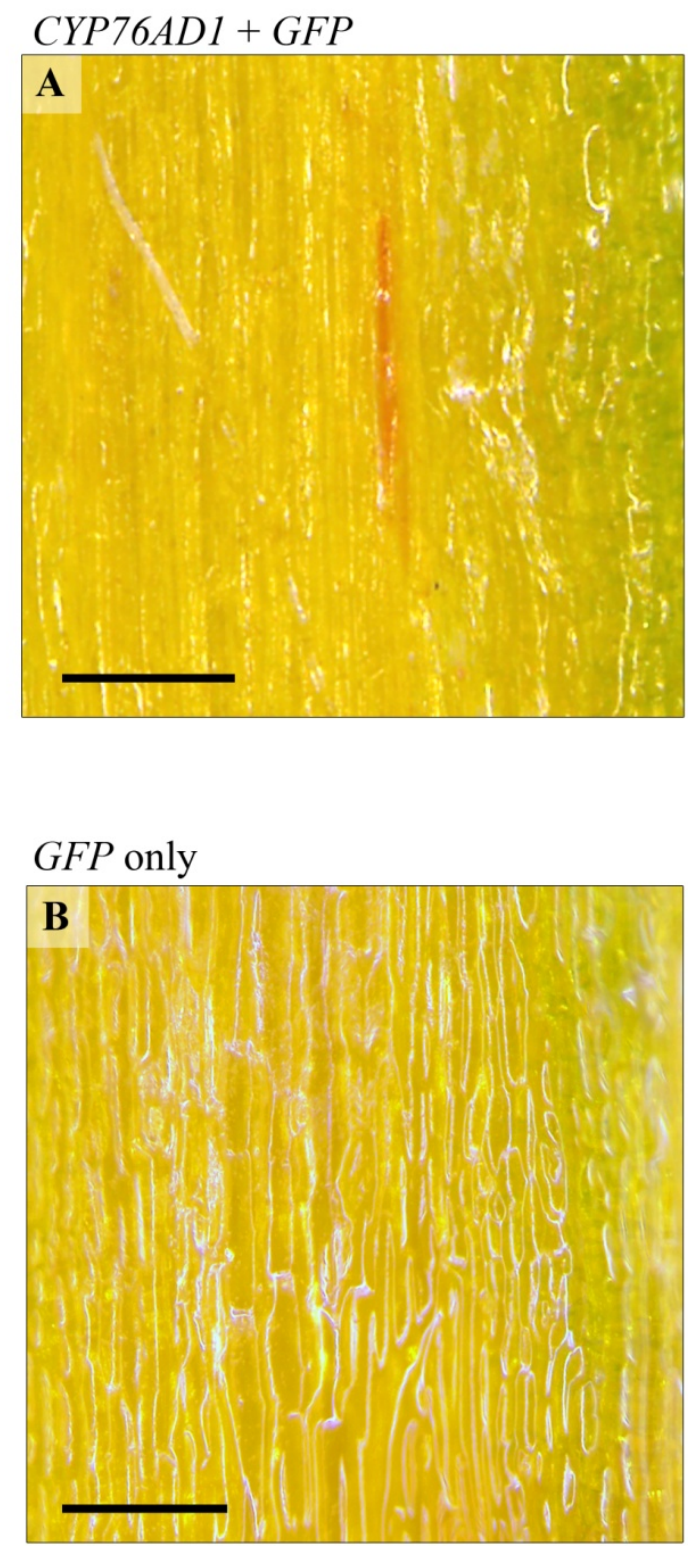

Figure 6.15 Transient gene expression of (A) CYP76AD1 + GFP and (B) GFP only constructs on petioles of yellow Swiss chard. Scale bars $=250 \mu \mathrm{m}$. 
Water
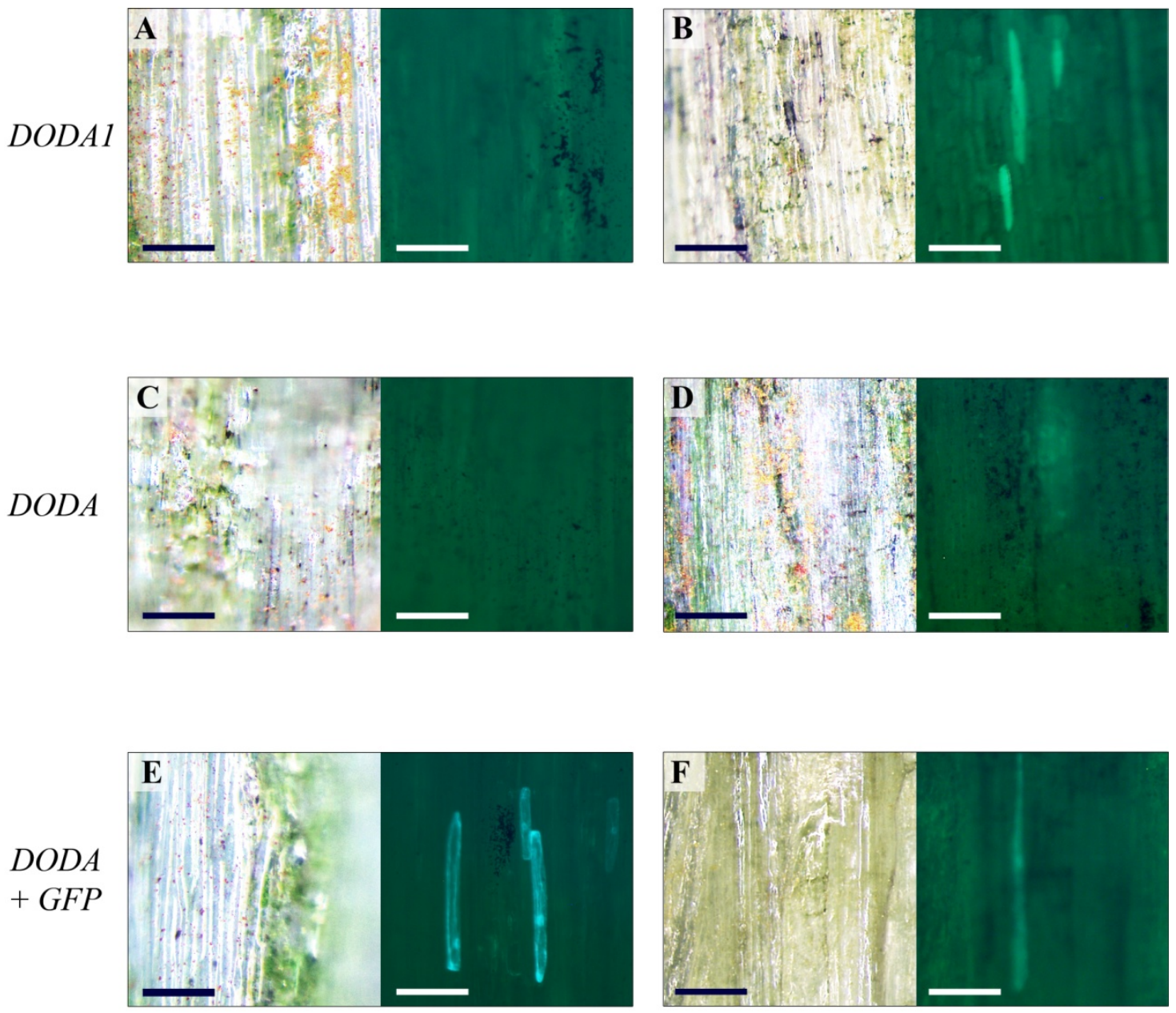

Figure 6.16 Transient expression of betalain biosynthetic genes in white Swiss chard petioles, vacuum infiltrated with water (left) and $10 \mathrm{mM}$ L-DOPA (right). Tissue was examined under white and blue light, (A and B) DODA1, (C and D) DODA, (E and F) $D O D A+G F P$. Scale bars $=200 \mu \mathrm{m}$. 


\subsection{Discussion}

\subsubsection{Regulation of betalain biosynthetic genes in response to wounding}

The main biosynthetic steps are known in the betalain pathway (Gandía-Herrero \& García-Carmona, 2013). Betalain biosynthetic enzymes, DODA1 and CYP76AD1, have been isolated and characterised (Hatlestad et al., 2012), although, there is debate over what enzyme controls the initial tyrosine hydroxylation reaction (Gandía-Herrero \& García-Carmona, 2013). Even though the betalain biosynthetic pathway appears quite simple, few studies have looked into how this pathway is regulated. There are only a few possible points for biosynthetic regulation in this pathway, given limited number of reactions controlled by enzymes compared to the number of spontaneous reactions. This study used the wounding response in Swiss chard leaves as a model for investigating betalain gene up-regulation, as physical wounding was previously demonstrated to trigger betalain production in beet leaves (Sepúlveda-Jiménez et al., 2004).

Betalain pigments visually accumulated around the wounding sites on the red-but not white-petioled leaf tissues (Figure 6.6), as expected. Gene expression of DODA1 and CYP76AD1, however, differed from expectations. In red line, both of these betalain biosynthetic genes were not up-regulated in response to wounding (Figure 6.11), even though these tissues visibly accumulated betalains. Further analysis of DODAl and CYP76AD1 revealed that these genes were already highly expressed at the time of wounding (Figure 6.12). In the negative betalain production control, the white line, $D O D A 1$ was also not up-regulated (Figure 6.11). However, CYP76AD1 was up-regulated after $24 \mathrm{~h}$ (Figure 6.11), although, relative transcript abundance changes were minor (Figure 6.12). The other putative $D O D$ gene, $D O D A$, was only up-regulated in response to wounding in white-petioled tissues (Figure 6.11). The DODA relative transcript abundance patterns differed from that of DODA1 and CYP76AD1 genes, with similar transcript levels in both red and white tissues (Figure 6.12). Given that gene expression patterns of known betalain biosynthetic genes did not match the production of betalains in the red line, betalain biosynthesis after wounding, at least in these tissues, is unlikely to be controlled by the up-regulation of $D O D$ or $C Y P 76 A D 1$ genes. In addition, differing expression patterns of DODA to DODA1 and CYP76AD1 suggest separate regulation of the $D O D A$ gene. 
Several different mechanisms are involved in the regulation of flavonoid biosynthesis, as discussed in Section 1.8. Flavonoid production is primarily through transcriptional control of pathway genes (Davies et al., 2012). Most commonly, regulation of these genes is from the binding of transcription factors, such as MYBs, to DNA elements upstream of the coding sequence. The regulation of flavonoid genes following physical wounding is a good example of this. Like betalains in beet, production of flavonoids can be induced by a physical wounding in a number of species (Djordjevic et al., 1997; Gould et al., 2002; Reyes \& Cisneros-Zevallos, 2003). In addition, wounding sites have been correlated with the up-regulation of flavonoid biosynthetic genes $C H S$ (Boava et al., 2011; Djordjevic et al., 1997; Mellway et al., 2009; Richard et al., 2000), F3H (Singh et al., 2008) and DFR (Peters \& Constabel, 2002), and may be regulated by wounding-specific MYB transcription factors (Cheong et al., 2002; Mellway et al., 2009).

Betalainic plants in the Caryophyllales, including beet, contain most of the major flavonoids, but lack anthocyanins (Bittrich \& Amaral, 1991; Georgiev et al., 2010; Giannasi, 1988; Iwashina, 2001; Kimler et al., 1970; Kujala et al., 2002; Martin, 1977; Pyo et al., 2004). To investigate whether betalain and flavonoid biosynthetic genes co-regulate in response to wounding, three flavonoid biosynthetic genes were monitored with RT-qPCR. In both the red- and white-petioled leaf tissue, CHS, UGT73A4 and $U G T 71 F 1$ genes were all up-regulated, with similar up-regulation patterns in the two lines (Figure 6.9). Given that betalain biosynthetic genes, DODA1 and CYP76AD1, were not up-regulated in response to wounding, but several flavonoid biosynthetic genes were, biosynthetic regulation of betalains may not use flavonoid regulatory control mechanisms. Rather, in this case, it appears that betalain biosynthesis is controlled either earlier in the pathway or post-transcriptionally.

In addition to monitoring expression of flavonoid glucosyltransferases for co-regulation with betalain genes, expression of $U G T 73 A 4$ and $U G T 71 F 1$ was monitored for the possible involvement in betacyanin biosynthesis. UGT73A4 and UGT71F1 are part of a large gene family (Vogt \& Jones, 2000). Phylogenetic alignments of plant glycosyltranferases $(G T \mathrm{~s})$ found that clades of $G T \mathrm{~s}$ conformed to whether plants produced anthocyanins or betalains (Isayenkova et al., 2006). This suggests betalain and anthocyanin biosynthesis uses separate and specific GTs. Betacyanin glycosylation has 
two possible routes, through the glycosylation of cyclo-DOPA (Heuer \& Strack, 1992; Heuer et al., 1996; Vogt et al., 1997) or betanidin glycosylation (Das et al., 2013; Sasaki et al., 2005a; Sasaki et al., 2004; Sasaki et al., 2005b; Sciuto et al., 1972; Sepulveda-Jimenez et al., 2005). UGT73A4 and UGT71F1 were both regioselective to flavonoids in vitro, but both enzymes had a low affinity for betanidin (Isayenkova et al., 2006).

Both GTs were up regulated in red Swiss chard tissues between $24 \mathrm{~h}$ and $48 \mathrm{~h}$ (Figure 6.8), coincident with the appearance of betalain pigments, which were visible by $48 \mathrm{~h}$ and continued to accumulate with time (Figure 6.6). Although these genes were up-regulated in response to wounding, they did not correlate with DODAl or CYP76AD1 expression in the red line (Figure 6.11). This indicates that separate biosynthetic control mechanisms are likely. In the biosynthesis of anthocyanins, up-regulation of GTs can be controlled independently of other flavonoid genes (Boss et al., 1996; Kobayashi et al., 2002). The separate regulatory control of GTs enables the plant to control the timing of coloured anthocyanin production, such as in developing fruit, separately from production of proanthocyanidins. However, betacyanins do not require glycosylation to become coloured compounds, although the majority of betalains found in Swiss chard are glycosylated.

Another possibility is that this gene is not involved in the glycosylation of betacyanins, and was instead up-regulated to glycosylate flavonoids in Swiss chard tissues. In the study by Sepulveda-Jimenez et al. (2005), a different putative betanidin GT was induced by wounding in beet. Also, Isayenkova et al. (2006) found that Beta vulgaris produced low levels of cyclo-DOPA glucoside, indicating the activity of a cyclo-DOPA GT. Both the putative betanidin and cyclo-DOPA GTs should be incorporated into future analyses.

\subsubsection{Comparison of DODA1 and DODA function}

$D O D$ genes in the Caryophyllales have homology to the LigB domain of bacterial extradiol 4,5-dioxygenases (Christinet et al., 2004). Two classes of LigB homologues have been found in plants, Class I and Class II. Class I genes are found both in the Caryophyllales and other taxa. Class II genes are presumed to be involved only in betalain biosynthesis, although recently, a possible Class II gene was found in Nicotiana benthamiana, an anthocyanin-producing species (Bahramnejad et al., 2010). In 
addition, several of these taxa, including Beta vulgaris, have two different $\operatorname{Lig} B$ genes annotated as DOD (Christinet et al., 2004; Hatlestad et al., 2012; Takahashi et al., 2009). Phylogenetic analyses of DOD place the two Beta vulgaris DOD genes into separate clades (Figure 6.13). To investigate the roles of DODA1 and DODA in betalain biosynthesis, transient gene expression analyses were carried out using Antirrhinum and Swiss chard tissues. From biolistic shooting into Antirrhinum, only DODAl was involved in production of betalains (Figure 6.14). DODA did not produce any visible pigmentation, only a non-coloured, autofluorescent compound present after L-DOPA feeding. Given that $D O D A$ was up-regulated in white Swiss chard tissues after wounding, this type of $D O D$ could be a stress response gene common to both anthocyanin- and betalain-producing taxa. In support of this, the $D O D A$-like gene in $N$. benthamiana, $N b D O D A L 1$, was up-regulated in response to wounding and bacterial infection (Bahramnejad et al., 2010).

\subsubsection{Biosynthetic control of betalains in yellow and white Swiss chard}

Betalain biosynthetic control in yellow and white Swiss chard tissue was then tested using transient gene expression. CYP76AD1 complemented yellow Swiss chard with the production of red cells at the transformation sites (Figure 6.15). This indicated that these plants either did not contain the active form of the CYP76AD1 allele or that the gene was switched off in this tissue. Given that yellow Swiss chard plants produced trace amounts of betacyanins in the lower organs (Table 3.4, Chapter 3), the latter is more likely.

The cause for lack of pigmentation in white Swiss chard tissues was also investigated. These plants produced purple pigmentation at the base of the hypocotyls and in the roots of seedlings (Figure 6.3), indicating that these tissues did not lack functional betalain biosynthetic genes. Also, both $D O D A 1$ and $C Y P 76 A D 1$ were expressed in white lamina tissues at low levels (Figure 6.12). Betalain biosynthesis in white tissues was not complemented with the feeding of L-DOPA alone (data not shown), but was complemented when supplied both L-DOPA and DODA1 (Figure 6.16). Given that fluorescent cells were only observed at the sites of gold bombardment in DODA1 transformed tissues fed with L-DOPA, it suggests that the lack of betalains in these tissues is caused by the absence of DODA1 activity and the precursor DOPA. Pigmentation in white Swiss chard could, therefore, be controlled through a 
combination of post-transcriptional regulation of betalain biosynthetic genes in addition to the control of DOPA levels.

DOPA levels could be controlled in Swiss chard tissues by the presence of a strong oxidiser such as tyrosinase. The review by Gandía-Herrero and García-Carmona (2013) discussed how without a reducing agent such as ascorbic acid, L-DOPA can be transformed by tyrosinase into $o$-DOPA-quinone, which then cyclises to form dopachrome. These tyrosine-mediated reactions could result in the reduced availability of L-DOPA for downstream betalain biosynthetic reactions in white Swiss chard, thus, effectively switching off the betalain biosynthetic pathway.

The regulation of betaxanthins versus betacyanins in Swiss chard could be a complex system. Pigmentation patterns in Swiss chard hypocotyls (Figure 3.12) demonstrated differing ratios of betaxanthins and betacyanins in neighbouring cells. The conjugation reactions of amines/amino acids or cyclo-DOPA with betalamic acid to form betaxanthins or betacyanins may just be simply 'spontaneous' in some tissues. Especially given that betalamic acid is only detected in tissues that also contain betaxanthins (Chapter 3 and Steglich and Strack (1990)). It is possible that there is physical separation of precursors through compartmentalisation in the cells, or that there is some type of genetic regulatory control.

Expression of different biosynthetic transcription factors control anthocyanin pigmentation of different tissues (as reviewed by Davies et al. (2012)). For example in flowers, complex pigmentation patterns result largely through the expression of different families of transcription factors. The interaction of R2R3MYB and bHLH transcription factors, and WD-Repeat proteins, form a 'MBW' complex which is central to the production of anthocyanins (Grotewold, 2006). Anthocyanin biosynthesis only occurs in tissues where the MBW complexes form. Differing activity of the individual transcription factors, especially of R2R3MYBs, can generate a variety of pigmentation patterns through tissue-specific expression. A similar set of tissue-specific regulators might operate in betalain-producing plants.

\subsubsection{Conclusions and future directions}

In the betalainic plant Swiss chard, flavonoid biosynthetic genes, $C H S$ and $G T$ s, did not co-regulate with betalain genes $D O D A 1$ and $C Y P 76 A D 1$ in response to wounding. 
Although flavonoid biosynthetic genes were up-regulated, betalain biosynthetic genes were already switched on before wounding and at higher levels in the red compared to white lines. Together with results from transient expression analyses, the data suggest that betalains and anthocyanins do not share the same regulatory control mechanisms. The control of betalain biosynthesis could possibly be due to the following factors, alone or in combination: (1) control of DOPA synthesis by the tyrosine-hydroxylating enzyme production or activity; (2) maintenance of DOPA levels through controlled degradation; (3) post-transcriptional regulation by DOD/CYP76AD1 activity. The following three experiments could used to test these hypotheses.

- Identify what tyrosine-hydroxylating enzyme gene is involved in betalain production in Swiss chard. One experiment could be to examine tyrosinase activity following pigment induction, and isolate the tyrosinase gene. Once the gene was isolated, regulatory patterns of this gene following wounding induction could be assessed.

- Test substrate availability in white Swiss chard by measuring DOPA and betalamic acid levels. Also, a comparison with neighbouring unpigmented tissues around wounding sites on red Swiss chard would be interesting to see if they contain these precursors.

- Test for post-translational control of the biosynthetic genes by measuring changes in betalainic proteins and associated compounds following betalain pigment induction.

In addition to those listed above, it would be essential to test whether the pattern of betalain biosynthetic regulation in Swiss chard is similar following other pigment induction methods, such as exposure to high light. A comparison could be made by monitoring of the same genes as used in this study, in addition to monitoring more flavonoid biosynthetic genes and betalain specific GTs. Although as found in this study, better house-keeping genes would be required. Identification of sequences in databases for other genes such as $F L S, D F R$ and $A N S$ were attempted in this study, but none were found in time to include them in RT-qPCR analyses. 


\section{Chapter 7: General discussion}

In this dissertation, I report a series of multidisciplinary studies that compare and contrast the production of betalain and anthocyanin pigments in plants. Betalain and anthocyanin plant pigments are similar in their appearance. It is due to these similarities that betalains were originally thought to be a class of anthocyanins. The discovery that betalains have an entirely separate biosynthetic pathway from anthocyanins (Kimler et al., 1970; Mabry \& Dreiding, 1967; Mabry et al., 1963) ignited interest in researching how betalain pigments evolved and why. Many studies in this area hypothesise that sometime in the evolutionary history of the Caryophyllales, betalains replaced anthocyanins (Brockington et al., 2011). Although widely assumed, the replacement hypothesis has not been thoroughly examined.

The localisation of betalain pigments to specific tissue and cell types support this replacement hypothesis. Histological analyses revealed that betalain-producing plants have similar pigmentation patterns to anthocyanin-producing plants. In vegetative organs, betalain pigments most frequently localised to specific dermal and ground tissues, which are similar to those reported for anthocyanins (Lee \& Collins, 2001). In addition, most of the betalain plants sectioned, as well as many anthocyanic species, have multiple pigmented organs on the same plant (Blank, 1947; Lee \& Collins, 2001; Thakur \& Nozzolillo, 1978). These similarities in pigment localisation of highly divergent species, those outside the Caryophyllales and within, suggest that anthocyanin and betalain pigment in particular tissues may have specific biological functions. However, there are only a limited number of studies which compare the physiological roles of betacyanins with anthocyanins. This area of research is important to future investigations into the replacement hypothesis.

The colour of betalains and anthocyanin pigments often appears similar, which is one of the main reasons why betalains are said to replace anthocyanins. However, the control over the colour of pigmentation is quite different. The cause of anthocyanin colouration tends to be from one major anthocyanin compound, with variation caused by co-pigmentation with other flavonoids such as flavonols, vacuolar $\mathrm{pH}$ and cell shape (Mol et al., 1998). Whereas for betalains, the colour is largely determined ratio of two discrete colour classes, betaxanthins and betacyanins. Histological analyses revealed 
that several betalain-producing plants have differential localisation of betacyanins and betaxanthins in neighbouring tissues. This type of pigmentation patterning has not been found in anthocyanic plants. The separation of betaxanthins from betacyanins in specific organs and tissues may also indicate that there are differing functional roles for these two classes of pigments. With the focus on comparing betacyanins with purple and red anthocyanins, no research has yet evaluated the potential functions of betaxanthins.

For future physiological experiments that compare the function of betalain and anthocyanin pigments, a model system was developed using an Arabidopsis thaliana transgenic line created by Harris et al. (2012). This transgenic Arabidopsis harbours the betalain biosynthetic gene DOPA extradiol dioxygenase (DOD) from Amanita muscaria, and is capable of producing betaxanthins when supplied L-DOPA. Methods for feeding L-DOPA were assessed. However, the ability for these plants to produce betaxanthins whilst remaining healthy was limited. It was determined that establishing betaxanthin-producing plants for physiological testing would only be successful using seedlings and for short term (i.e. less than a week) analyses. This betalain model system was also limited by the fact that it could only produce betaxanthins. The spectral properties of anthocyanins are different to those of betaxanthins. For direct physiological comparisons of anthocyanins to betalains, betacyanins would need to be produced in Arabidopsis. Production of betacyanin was found to be possible through the feeding of cyclo-DOPA in addition to L-DOPA. However, cyclo-DOPA feeding was inefficient and negatively impacted plant health.

To work around the issues with feeding cyclo-DOPA, a DOD Arabidopsis line was transformed with the betalain biosynthetic gene from Beta vulgaris, CYP76AD1. It is possible, therefore, that in future experiments the DOD/CYP76AD1 Arabidopsis plants could produce both classes of betalains. However, these analyses would again be limited by the liquid feeding of L-DOPA. Recently, the biosynthesis of betaxanthins in Arabidopsis cell cultures was achieved by transforming these cells with both a tyrosinase and a DOD (Nakatsuka et al., 2013). If a similar tyrosinase was inserted into DOD or DOD/CYP76AD1 Arabidopsis lines, there is potential to create a betalain-producing Arabidopsis line without needing to feed L-DOPA. However, betalain production may not be stable with constitutive expression of a tyrosinase given that the cell lines created by Nakatsuka et al. (2013) went brown with time. 
An alternative hypothesis to explain mutual exclusivity of anthocyanins and betalains is that co-existence of the two pigment types negatively affects plant health. To test co-compatibility, Arabidopsis thaliana lines were developed to produce betaxanthins alone when fed L-DOPA (DOD-6), strongly accumulate anthocyanins (PAP1-5), or both betaxanthins and anthocyanins (DOD-6 x PAP1-5). DOD-6 x PAP1-5 was the first known co-production of anthocyanins and betalains in the same plant. These plants strongly accumulated betaxanthins despite the tissue already containing anthocyanins. However, betaxanthins do not appear to be stable in Arabidopsis tissues. In DOD-6 plants there was a loss of betaxanthins with time, even with continued L-DOPA feeding. Also, there appeared to be an absence of epidermal betaxanthins in the DOD-6 x PAP-1 line after L-DOPA feeding. It is, therefore, possible that a physiological barrier still exists in anthocyanic plants, such as Arabidopsis, that prevents stable betalain accumulation. Further examination of betalain stability in Arabidopsis is essential to future experiments testing the replacement hypothesis.

The replacement hypothesis was supported by the transport analyses of betalains. Using the Arabidopsis model system described above, known anthocyanin transporters were tested for their involvement in betaxanthin accumulation. Both betalain and anthocyanin biosynthetic enzymes are thought to be localised on the cytoplasmic side of the endoplasmic reticulum (ER) (Gandía-Herrero \& García-Carmona, 2012; Hrazdina et al., 1978). Therefore, they both require intracellular transport from site of synthesis to storage in the vacuole. In the synthesis of betalains, the conjugation reactions between betalamic acid and amino acids or cyclo-DOPA to form betaxanthins or betacyanins, respectively, occur spontaneously. Given the spontaneous nature of these reactions, it was hypothesised by Trezzini and Zyrd (1990) that betalamic acid is transported to the vacuole for the formation of betaxanthins. This was proposed because Portulaca grandiflora has the locus $I$ (inhibitor) that prevents the condensation of amino acids with betalamic acid. In support of the conjugation reaction occurring in the vacuole, Schliemann et al. (1999) demonstrated that the vacuole contains similar distribution and concentration of amino acids to the cytoplasm. However, it is unknown whether there are single or multiple pools of betalamic acid (Grotewold, 2006). The separation of different betalamic acid pools may play a role in controlling betaxanthin biosynthesis. Examination of L-DOPA fed DOD-6 Arabidopsis tissues, however, provided evidence that betaxanthins can form outside the vacuole. As this is an artificial 
betalain-producing system, the formation of betaxanthins outside of the vacuole would need to be confirmed in natural betalain-producing plants.

Direct comparisons of transport mechanisms for betaxanthins and anthocyanins were possible by using known transport inhibitors and through the genetic crossing of DOD-6 with two flavonoid transport mutant lines: $t t 12$, a multidrug and toxic extrusion (MATE) transporter mutant (DOD-6 x tt12), and $t t 19$, a glutathione $S$-transferase (GST) mutant (DOD-6 x tt19). Betaxanthin transport mechanisms in Arabidopsis shared most of those used by anthocyanins. Both anthocyanins (Poustka et al., 2007) and betalains appear to use a direct transport route from the ER to storage in the vacuole. Betaxanthin accumulation was not affected by the Golgi-disturbing agent, Brefeldin A (BFA), indicating that these compounds do not pass through the trans-Golgi network. Also similar to anthocyanins, vesicles may be involved in betalain transport. Vesicular-like structures were observed in tissues accumulating betaxanthin. It is hypothesised that these vesicle-like structures containing betaxanthins may be involved with mass-transport of the pigments to the vacuole.

GST-like transporters are another transport mechanism that betaxanthins and anthocyanins may share. The $t t 19$ mutant in Arabidopsis has reduced ability to accumulate anthocyanins in vegetative tissues (Kitamura et al., 2004; Sun et al., 2012). The betalain-producing GST mutant, DOD-6 x tt19, had strong reductions in betaxanthin accumulation. These pigments appeared to be absent in the vacuoles of all cells except in guard cells, which may have redundant vacuolar transport mechanisms (Thompson et al., 2010). Confocal analyses revealed that betaxanthins were restricted to the cytoplasm in these tissues, indicating that TT19 is essential for betaxanthins to cross the tonoplast.

An ATP-binding cassette (ABC) transporter is also involved in Arabidopsis betaxanthin accumulation. Treatment with the general ATPase inhibitor vanadate (VAN), reduced vacuolar accumulation in L-DOPA fed DOD-6. These results are similar to those published for anthocyanin accumulation in Arabidopsis (Poustka et al., 2007). The ATP-activated transporter involved in betalain biosynthesis may be a glutathione (GS-X) pump, as vanadate treated cells had similar phenotypes to the DOD-6 x tt19 mutant. 
Another transporter that is involved in betaxanthin-accumulation in Arabidopsis is the proanthocyanidin TT12 MATE transporter. Pigmentation was reduced in L-DOPA fed DOD- 6 x $t t 12$ but anthocyanin pigmentation was not affected in the $t t 12$ line (Debeaujon et al., 2001). In contrast, the chemical MATE inhibitor, pyrimethamine (PYR), had no affect on betalain accumulation but induced anthocyanic vacuolar inclusions (AVIs) to form in DOD-6 x PAP1-5 tissues. These results suggest that two different MATE transporters may be involved in flavonoid transport in Arabidopsis: one specific to proanthocyanidin transport and the other for anthocyanin transport, with only the former able to transport betaxanthins.

Overall, it appears that betalains and anthocyanins share transport mechanisms, at least in this artificial pigment system. Further elucidation of how each transporter may function in the transport of these pigments is required, as well as whether similar mechanisms are involved in natural betalain-producing plants.

The biosynthetic regulation of betalain pigments is unknown. Given the similarities of betalain and anthocyanin induction, localisation and possible transport mechanisms, it was hypothesised that betalain biosynthesis uses aspects of the flavonoid regulatory pathway. However, the expression patterns of flavonoid and betalain biosynthetic genes differed following wounding induction in the betalain-producing plant, Swiss chard (Beta vulgaris subsp. cicla cv. 'Bright Lights').

Production of betalains was clearly induced around the wounding sites on red-petioled leaves (Figure 6.6). Previous reports also demonstrated wounding induced the production of anthocyanins and flavonoids in anthocyanic species (Gould et al., 2002; Reyes \& Cisneros-Zevallos, 2003). However, core betalain biosynthetic genes DODA1 and $C Y P 76 A D 1$ were not up-regulated in the red line, whereas, flavonoid biosynthetic genes were. Further analysis of $D O D A 1$ and $C Y P 76 A D 1$ revealed that they were both highly expressed prior to wounding. Since there are only three main biosynthetic steps controlled by enzymes in the betalain pathway, it is possible that regulation of betalain biosynthesis occurs earlier in the pathway, with the tyrosine hydroxylating enzyme. Alternatively, betalain biosynthesis may have evolved separate regulatory mechanisms to those in the flavonoid pathway, at least in response to wounding in Swiss chard. 
The differential localisation of betacyanins and betaxanthins observed in the histological analyses also gives us insight into betalain biosynthesis. Biosynthesis of betacyanins and betaxanthins are linked, as they both contain the chromophore betalamic acid (Girod \& Zrÿd, 1991). The uneven distribution if these pigments suggest independent regulation of betaxanthin and betacyanin compound production. Combined with the RT-qPCR data, these results indicate that there may be more than one point of biosynthetic control in the betalain pathway, at the tyrosine hydroxylating enzyme step, and after the production of betalamic acid.

Betacyanin production in yellow Swiss chard petioles was complemented by the transient gene expression of CYP76AD1. In the pigment analyses in Chapter 3, Yellow Swiss chard organs contained traces of betacyanins in yellow organs which indicates that these plants were not $C Y P 76 A D 1$ mutants. Therefore, the lack of betacyanin production in these tissues may be due to down-regulation of $C Y P 76 A D 1$.

The biosynthetic control in white tissues appears more complex than that of yellow tissues. Pigment analyses of white Swiss chard found that betacyanins were produced in low quantities in the stem and at the base of the petiole, which indicates that these tissues had functional betalain biosynthetic genes. In addition, the RT-qPCR analyses of white Swiss chard revealed that white tissues had low level expression of both DODAI and CYP76AD1. However, betaxanthin production in white petioles was not complemented with the feeding of the precursor L-DOPA, or the transient expression of DODA1, by themselves. Betaxanthins were only produced with the combined transient expression of DODA1 in the presence of L-DOPA. These results suggest two ways of biosynthetic control in white tissues. Firstly, expression of $D O D A 1$ may be down-regulated, with the endogenous level of expression too low to produce betalains when supplied DOPA. Secondly, these tissues may be precursor-limited given that betalains weren't produced with the transient expression of $D O D A 1$. Strong oxidation of L-DOPA was observed in white tissues following vacuum infiltration. It is, therefore, possible that the regulation of betalain biosynthesis in white Swiss chard is controlled, in part, by degradation of the precursor, DOPA. An over-active tyrosinase or other oxidising enzyme may be the cause of this degradation (Gandía-Herrero \& GarcíaCarmona, 2013). 
Together, the analyses of biosynthetic control in different coloured Swiss chard lines suggest that a variety of factors are involved in the regulation of betalain production. For future betalain biosynthetic control experiments, it would be important to test whether betalain gene expression patterns are the same following other induction methods such as high light. In addition, analyses should focus on post-transcriptional control of betalain biosynthesis to elucidate where in the pathway betalain biosynthesis is controlled.

This study has also provided insight into the evolution of betalain pigments. It is possible that during the evolution of betalain pigments, pre-existing transport and induction mechanisms involved in anthocyanin production were taken over to produce betalains. That way, only a few enzymatic steps were required for a new biosynthetic pathway to form. Betalain biosynthetic enzymes may also have evolved from enzymes already present in the ancestral species (Brockington et al., 2011). For example, DOPA extradiol dioxygenase (DOD), which is involved in the synthesis of betalamic acid, is part of a large family of LigB homologues. Christinet et al. (2004) found that there are homologues to the betalain DOD in taxa outside of the Caryophyllales. In addition, two DODs have been identified in a number of betalain-producing plants (Christinet et al., 2004; Hatlestad et al., 2012; Takahashi et al., 2009), including B. vulgaris. It has been assumed that the two LigB genes that have been reported in betalain species are both betalain related (Christinet et al., 2004; Hatlestad et al., 2012; Takahashi et al., 2009). In $B$. vulgaris, the $D O D$ gene $D O D A 1$ is known to be involved in betalain biosynthesis (Hatlestad et al., 2012) and recombinant DODA from E. coli synthesised betalamic acid from DOPA in in vitro assays (Gandía-Herrero \& García-Carmona, 2012).

The Swiss chard RT-qPCR experiment revealed that $D O D A$ was up-regulated in response to wounding but only in white Swiss chard tissues, and DODAl was not up-regulated at all. These two DODs may have different functions in vivo. In the white line, $D O D A 1$ and not $D O D A$ appeared to complement betaxanthin biosynthesis. In contrast, $D O D A$ expression in the presence of L-DOPA produced an unidentified fluorescent product. Given that DODA does not seem to be involved in betalain biosynthesis but yet was up-regulated in response to wounding, may indicate that this is a stress-response gene. A similar gene has been found in the anthocyanin-producing species Nicotiana benthamiana, and was also up-regulated in response to wounding 
(Bahramnejad et al., 2010). Given that the in vitro results by Gandía-Herrero and García-Carmona (2012) differed from the in vivo results in this study, the catalytic properties of these two enzymes may be different in vivo. Together, these results 


\section{References}

Adams, P., Nelson, D. E., Yamada, S., Chmara, W., Jensen, R. G., Bohnert, H. J., \& Griffiths, H. (1998). Growth and development of Mesembryanthemum crystallinum (Aizoaceae). New Phytologist, 138(2), 171-190.

Aharoni, A., De Vos, C., Wein, M., Sun, Z., Greco, R., Kroon, A., Mol, J. N., \& O'Connell, A. P. (2001). The strawberry FaMYB1 transcription factor suppresses anthocyanin and flavonol accumulation in transgenic tobacco. The Plant Journal, 28(3), 319-332.

Ahmad, M., Lin, C., \& Cashmore, A. R. (1995). Mutations throughout an Arabidopsis blue-light photoreceptor impair blue-light-responsive anthocyanin accumulation and inhibition of hypocotyl elongation. The Plant Journal, 8(5), 653-658.

Albert, N. W., Lewis, D. H., Zhang, H., Irving, L. J., Jameson, P. E., \& Davies, K. M. (2009). Light-induced vegetative anthocyanin pigmentation in Petunia. Journal of Experimental Botany, 60(7), 2191-2202.

Albert, N. W., Lewis, D. H., Zhang, H., Schwinn, K. E., Jameson, P. E., \& Davies, K. M. (2011). Members of an R2R3-MYB transcription factor family in Petunia are developmentally and environmentally regulated to control complex floral and vegetative pigmentation patterning. The Plant Journal, 65(5), 771-784.

Alfenito, M. R., Souer, E., Goodman, C. D., Buell, R., Mol, J., Koes, R., \& Walbot, V. (1998). Functional complementation of anthocyanin sequestration in the vacuole by widely divergent glutathione S-transferases. The Plant Cell 10(7), 11351149.

Alonso, J. M., Stepanova, A. N., Leisse, T. J., Kim, C. J., Chen, H., Shinn, P., Stevenson, D. K., Zimmerman, J., Barajas, P., \& Cheuk, R. (2003). Genomewide insertional mutagenesis of Arabidopsis thaliana. Science Signaling, 301(5633), 653.

Arvidsson, S., Kwasniewski, M., Riaño-Pachón, D. M., \& Mueller-Roeber, B. (2008). QuantPrime - a flexible tool for reliable high-throughput primer design for quantitative PCR. BMC Bioinformatics, 9(1), 465.

Azeredo, H. M. C. (2009). Betalains: properties, sources, applications, and stability - a review. International Journal of Food Science \& Technology, 44(12), 23652376.

Bahramnejad, B., Erickson, L. R., \& Goodwin, P. H. (2010). Induction of expression and increased susceptibility due to silencing a 4,5-DOPA dioxygenase extradiollike gene of Nicotiana benthamiana in the interaction with the hemibiotrophic pathogens, Colletotrichum destructivum, Colletotrichum orbiculare or Pseudomonas syringae pv. tabaci. Plant Science, 178(2), 147-157.

Bhuiyan, M. N. H., \& Adachi, T. (2003). Stimulation of betacyanin synthesis through exogenous methyl jasmonate and other elicitors in suspension-cultured cells of Portulaca. Journal of Plant Physiology, 160(9), 1117-1124.

Bittrich, V., \& Amaral, M. D. C. E. (1991). Proanthocyanidins in the testa of centrospermous seeds. Biochemical Systematics and Ecology, 19(4), 319-321.

Blank, F. (1947). The anthocyanin pigments of plants. Botanical Review, 13(5), 241317.

Boava, L. P., Cristofani-Yaly, M., Stuart, R. M., \& Machado, M. A. (2011). Expression of defense-related genes in response to mechanical wounding and Phytophthora parasitica infection in Poncirus trifoliata and Citrus sunki. Physiological and Molecular Plant Pathology, 76(2), 119-125. 
Bohm, H., \& Mack, G. (2004). Betaxanthin formation and free amino acids in hairy roots of Beta vulgaris var. lutea depending on nutrient medium and glutamate or glutamine feeding. Phytochemistry, 65(10), 1361-1368.

Bokern, M., Heuer, S., \& Strack, D. (1992). Hydroxycinnamic acid transferases in the biosynthesis of acylated betacyanins: purification and characterization from cell cultures of Chenopodium rubrum and occurrence in some other members of the Caryophyllales. Botanica Acta, 105(3), 146-151.

Bokern, M., \& Strack, D. (1988). Synthesis of hydroxycinnamic acid esters of betacyanins via 1-O-acylglucosides of hydroxycinnamic acids by protein preparations from cell suspension cultures of Chenopodium rubrum and petals of Lampranthus sociorum. Planta, 174(1), 101-105.

Boss, P. K., Davies, C., \& Robinson, S. P. (1996). Analysis of the expression of anthocyanin pathway genes in developing Vitis vinifera L. cv Shiraz grape berries and the implications for pathway regulation. Plant Physiology, 111(4), 1059-1066.

Bradley, J. M., Davies, K. M., Deroles, S. C., Bloor, S. J., \& Lewis, D. H. (1998). The maize $L c$ regulatory gene up-regulates the flavonoid biosynthetic pathway of Petunia. The Plant Journal, 13(3), 381-392.

Brockington, S. F., Walker, R. H., Glover, B. J., Soltis, P. S., \& Soltis, D. E. (2011). Complex pigment evolution in the Caryophyllales. New Phytologist, 190(4), 854-864.

Cai, Sun, M., \& Corke, H. (2001). Identification and distribution of simple and acylated betacyanins in the Amaranthaceae. Journal of Agricultural and Food Chemistry, 49(4), 1971-1978.

Cai, Y., \& Corke, H. (1999). Amaranthus betacyanin pigments applied in model food systems. Journal of Food Science, 64(5), 869-873.

Cai, Y. Z., Sun, M., \& Corke, H. (2003). Antioxidant activity of betalains from plants of the Amaranthaceae. Journal of Agricultural and Food Chemistry, 51(8), 22882294.

Cao, S., Liu, T., Jiang, Y., He, S., Harrison, D. K., \& Joyce, D. C. (2012). The effects of host defence elicitors on betacyanin accumulation in Amaranthus mangostanus seedlings. Food Chemistry, 134(4), 1715-1718.

Chalker-Scott, L. (1999). Environmental significance of anthocyanins in plant stress responses. Photochemistry and Photobiology, 70(1), 1-9.

Chalker-Scott, L. (2002). Do anthocyanins function as osmoregulators in leaf tissues? Advances in Botanical Research (Vol. 37, pp. 103-127): Academic Press.

Chang-Quan, W., \& Tao, L. (2006). Cryptochrome 2 is involved in betacyanin decomposition induced by blue light in Suaeda salsa. Functional Plant Biology, 33(7), 697.

Chen, X. W., Shao, L., Song, L. Y., Chen, Y. J., Peng, C. L., \& Zhang, Q. (2013). Amaranthine plays an important role in photoprotection for Alternanthera sessilis under photooxidative stress. Biotechnology \& Biotechnological Equipment, 27(3), 3791-3797.

Cheong, Y. H., Chang, H.-S., Gupta, R., Wang, X., Zhu, T., \& Luan, S. (2002). Transcriptional profiling reveals novel interactions between wounding, pathogen, abiotic stress, and hormonal responses in Arabidopsis. Plant Physiology, 129(2), 661-677.

Chinnusamy, V., Ohta, M., Kanrar, S., Lee, B. H., Hong, X., Agarwal, M., \& Zhu, J. K. (2003). ICE1: a regulator of cold-induced transcriptome and freezing tolerance in Arabidopsis. Genes \& Development, 17(8), 1043-1054. 
Christie, P. J., Alfenito, M. R., \& Walbot, V. (1994). Impact of low-temperature stress on general phenylpropanoid and anthocyanin pathways: Enhancement of transcript abundance and anthocyanin pigmentation in maize seedlings. Planta, 194(4), 541-549.

Christinet, L., Burdet, F. X., Zaiko, M., Hinz, U., \& Zrÿd, J.-P. (2004). Characterization and functional identification of a novel plant 4, 5-extradiol dioxygenase involved in betalain pigment biosynthesis in Portulaca grandiflora. Plant Physiology, 134(1), 265-274.

Chung, M.-H., Chen, M.-K., \& Pan, S.-M. (2000). Floral spray transformation can efficiently generate Arabidopsis. Transgenic Research, 9(6), 471-486.

Clement, J., \& Mabry, T. (1996). Pigment evolution in the Caryophyllales: a systematic overview. Botanica Acta, 109, 360-367.

Close, D., \& Beadle, C. (2003). The ecophysiology of foliar anthocyanin. The Botanical Review, 69(2), 149-161.

Clough, S. J., \& Bent, A. F. (1998). Floral dip: a simplified method for Agrobacterium-mediated transformation of Arabidopsis thaliana. The Plant Journal, 16(6), 735-743.

Conn, S., Franco, C., \& Zhang, W. (2010). Characterization of anthocyanic vacuolar inclusions in Vitis vinifera L. cell suspension cultures. Planta, 231(6), 13431360 .

Das, S. S., Gauri, S. S., Misra, B. B., Biswas, M., \& Dey, S. (2013). Purification and characterization of a betanidin glucosyltransferase from Amaranthus tricolor L catalyzing non-specific biotransformation of flavonoids. Plant Science, 211, 6169.

Davies, K. M. (2009). Modifying anthocyanin production in flowers. In K. Gould, K. M. Davies \& C. Winefield (Eds.), Anthocyanins: Biosynthesis, Functions, and Applications (pp. 49-80). New York: Springer.

Davies, K. M., Albert, N. W., \& Schwinn, K. E. (2012). From landing lights to mimicry: the molecular regulation of flower colouration and mechanisms for pigmentation patterning. Functional Plant Biology, 39(8), 619-638.

Davies, K. M., \& Schwinn, K. E. (2003). Transcriptional regulation of secondary metabolism. Functional Plant Biology, 30(9), 913-925.

de Nicola, M. G., Amico, V., Sciuto, S., \& Piattelli, M. (1975). Light control of amaranthin synthesis in isolated Amaranthus cotyledons. Phytochemistry, 14(2), 479-481.

Debeaujon, I., Peeters, A. J. M., Léon-Kloosterziel, K. M., \& Koornneef, M. (2001). The TRANSPARENT TESTA12 gene of Arabidopsis encodes a multidrug secondary transporter-like protein required for flavonoid sequestration in vacuoles of the seed coat endothelium. The Plant Cell, 13(4), 853-871.

Dinter, A., \& Berger, E. (1998). Golgi-disturbing agents. Histochemistry and Cell Biology, 109(5-6), 571-590.

Djordjevic, M. A., Mathesius, U., Arioli, T., Weinman, J. J., \& Gärtner, E. (1997). Chalcone synthase gene expression in transgenic subterranean clover correlates with localised accumulation of flavonoids. Functional Plant Biology, 24(2), 119-132.

Dominy, N. J., Lucas, P. W., Ramsden, L. W., Riba-Hernandez, P., Stoner, K. E., \& Turner, I. M. (2002). Why are young leaves red? Oikos, 98(1), 163-176.

Ehrendorfer, F. (1976). Closing remarks: Systematics and evolution of centrospermous families. Plant Systematics and Evolution, 126(1), 99-106. 
El-Kereamy, A., Chervin, C., Roustan, J.-P., Cheynier, V., Souquet, J.-M., Moutounet, M., Raynal, J., Ford, C., Latché, A., Pech, J.-C., \& Bouzayen, M. (2003). Exogenous ethylene stimulates the long-term expression of genes related to anthocyanin biosynthesis in grape berries. Physiologia Plantarum, 119(2), 175182.

Esau, K. (1965). Plant Anatomy (2nd ed.). United States of America: John Wiley \& Sons, Inc.

Escribano, J., Pedreno, M. A., Garcia-Carmona, F., \& Munoz, R. (1998). Characterization of the antiradical activity of betalains from Beta vulgaris L. roots. Phytochemical Analysis, 9(3), 124-127.

Etxeberria, E., Pozueta-Romero, J., \& Gonzalez, P. (2012). In and out of the plant storage vacuole. Plant Science, 190, 52-61.

Foyer, C. H., Lelandais, M., \& Kunert, K. J. (1994). Photooxidative stress in plants. Physiologia Plantarum, 92(4), 696-717.

Francisco, R. M., Regalado, A., Ageorges, A., Burla, B. J., Bassin, B., Eisenach, C., Zarrouk, O., Vialet, S., Marlin, T., \& Chaves, M. M. (2013). ABCC1, an ATP binding cassette protein from grape berry, transports anthocyanidin 3-Oglucosides. The Plant Cell 25(5), 1840-1854.

Gandía-Herrero, F., Escribano, J., \& García-Carmona, F. (2005a). Betaxanthins as substrates for tyrosinase. An approach to the role of tyrosinase in the biosynthetic pathway of betalains. Plant Physiology, 138(1), 421-432.

Gandía-Herrero, F., Escribano, J., \& García-Carmona, F. (2010). Structural implications on color, fluorescence, and antiradical activity in betalains. Planta, 232(2), 449460.

Gandía-Herrero, F., \& García-Carmona, F. (2012). Characterization of recombinant Beta vulgaris 4,5-DOPA-extradiol-dioxygenase active in the biosynthesis of betalains. Planta, 236(1), 91-100.

Gandía-Herrero, F., \& García-Carmona, F. (2013). Biosynthesis of betalains: yellow and violet plant pigments. Trends in Plant Science, 18(6), 334-343.

Gandía-Herrero, F., García-Carmona, F., \& Escribano, J. (2005b). Fluorescent pigments: New perspectives in betalain research and applications. Food Research International, 38(8-9), 879-884.

Gandía-Herrero, F., Jimenez-Atienzar, M., Cabanes, J., Escribano, J., \& GarcíaCarmona, F. (2009). Fluorescence detection of tyrosinase activity on dopaminebetaxanthin purified from Portulaca oleracea (common purslane) flowers. Journal of Agricultural and Food Chemistry, 57(6), 2523-2528.

Gandía-Herrero, F., Jimenez-Atienzar, M., Cabanes, J., García-Carmona, F., \& Escribano, J. (2005c). Differential activation of a latent polyphenol oxidase mediated by sodium dodecyl sulfate. Journal of Agricultural and Food Chemistry, 53(17), 6825-6830.

Gandía-Herrero, F., Jimenez-Atienzar, M., Cabanes, J., García-Carmona, F., \& Escribano, J. (2005d). Evidence for a common regulation in the activation of a polyphenol oxidase by trypsin and sodium dodecyl sulfate. Biological Chemistry, 386(6), 601-607.

The Gene Index Databases. Retrieved 17/09/2013, 2013, from http://compbio.dfci.harvard.edu/tgi/

Georgiev, V., Weber, J., Kneschke, E.-M., Denev, P., Bley, T., \& Pavlov, A. (2010). Antioxidant activity and phenolic content of betalain extracts from intact plants and hairy root cultures of the red beetroot Beta vulgaris cv. Detroit dark red. Plant Foods for Human Nutrition, 65(2), 105-111. 
Giannasi, D. (1988). Flavonoids and evolution in the dicotyledons. In J. B. Harborne (Ed.), The Flavonoids: Advances in Research since 1980 (pp. 479-504). USA: Springer

Gillbro, T., \& Cogdell, R. J. (1989). Carotenoid fluorescence. Chemical Physics Letters, $158(3-4), 312-316$.

Girod, P. A., \& Zrÿd, J. P. (1991). Secondary metabolism in cultured red beet (Beta vulgaris L.) cells - differential regulation of betaxanthin and betacyanin biosynthesis. Plant Cell Tissue and Organ Culture, 25(1), 1-12.

Gleave, A. P. (1992). A versatile binary vector system with a T-DNA organisational structure conducive to efficient integration of cloned DNA into the plant genome. Plant Molecular Biology, 20(6), 1203-1207.

Goldman, I. L., \& Austin, D. (2000). Linkage among the $R, Y$ and $B l$ loci in table beet. Theoretical and Applied Genetics, 100(3-4), 337-343.

Goldsbrough, A., Belzile, F., \& Yoder, J. I. (1994). Complementation of the tomato anthocyanin without (aw) mutant using the dihydroflavonol 4-reductase gene. Plant Physiology, 105(2), 491-496.

Gomez, C., Conejero, G., Torregrosa, L., Cheynier, V., Terrier, N., \& Ageorges, A. (2011). In vivo grapevine anthocyanin transport involves vesicle-mediated trafficking and the contribution of anthoMATE transporters and GST. The Plant Journal, 67(6), 960-970.

Gomez, C., Terrier, N., Torregrosa, L., Vialet, S., Fournier-Level, A., Verriès, C., Souquet, J.-M., Mazauric, J.-P., Klein, M., \& Cheynier, V. (2009). Grapevine MATE-type proteins act as vacuolar $\mathrm{H}^{+}$-dependent acylated anthocyanin transporters. Plant Physiology, 150(1), 402-415.

Goodman, C. D., Casati, P., \& Walbot, V. (2004). A multidrug resistance-associated protein involved in anthocyanin transport in Zea mays. The Plant Cell, 16(7), 1812-1826.

Gould, K. S., Dudle, D. A., \& Neufeld, H. S. (2010). Why some stems are red: cauline anthocyanins shield photosystem II against high light stress. Journal of Experimental Botany, 61(10), 2707-2717.

Gould, K. S., McKelvie, J., \& Markham, K. R. (2002). Do anthocyanins function as antioxidants in leaves? Imaging of $\mathrm{H}_{2} \mathrm{O}_{2}$ in red and green leaves after mechanical injury. Plant, Cell \& Environment, 25(10), 1261-1269.

Gould, K. S., \& Quinn, B. D. (1999). Do anthocyanins protect leaves of New Zealand native species from UV-B? New Zealand Journal of Botany, 37, 175-178.

Grabov, A., \& Blatt, M. R. (1998). Co-ordination of signalling elements in guard cell ion channel control. Journal of Experimental Botany, 49(Special Issue), 351360.

Grotewold, E. (2004). The challenges of moving chemicals within and out of cells: insights into the transport of plant natural products. Planta, 219(5), 906-909.

Grotewold, E. (2006). The genetics and biochemistry of floral pigments. Annual Review of Plant Biology, 57, 761-780.

Grotewold, E., Chamberlin, M., Snook, M., Siame, B., Butler, L., Swenson, J., Maddock, S., Clair, G. S., \& Bowen, B. (1998). Engineering secondary metabolism in maize cells by ectopic expression of transcription factors. The Plant Cell 10(5), 721-740.

Grotewold, E., \& Davies, K. (2008). Trafficking and sequestration of anthocyanins. Natural Product Communications, 3(8), 1251-1258.

Guindon, S., \& Gascuel, O. (2003). A simple, fast, and accurate algorithm to estimate large phylogenies by maximum likelihood. Systematic Biology, 52(5), 696-704. 
Harris, N. N., Javellana, J., Davies, K. M., Lewis, D. H., Jameson, P. E., Deroles, S. C., Calcott, K. E., Gould, K. S., \& Schwinn, K. E. (2012). Betalain production is possible in anthocyanin-producing plant species given the presence of DOPAdioxygenase and L-DOPA. BMC Plant Biology, 12, 34.

Hatier, J.-H. B., \& Gould, K. S. (2009). Anthocyanin function in vegetative organs. In C. Winefield, K. Davies \& K. Gould (Eds.), Anthocyanins: Biosynthesis, Functions, and Applications (pp. 1-19). New York: Springer.

Hatlestad, G. J. (2012). Identification of the classical beet pigment loci, $R$ and $Y$, using whole genome profiling. Paper presented at the Plant and Animal Genome XX Conference (January 14-18, 2012).

Hatlestad, G. J., Sunnadeniya, R. M., Akhavan, N. A., Gonzalez, A., Goldman, I. L., McGrath, J. M., \& Lloyd, A. M. (2012). The beet $R$ locus encodes a new cytochrome P450 required for red betalain production. Nature Genetics, 44(7), 816-820.

Hayakawa, K., \& Agarie, S. (2010). Physiological roles of betacyanin in a halophyte, Suaeda japonica Makino. Plant Production Science, 13(4), 351-359.

Hays, J. B. (2002). Arabidopsis thaliana, a versatile model system for study of eukaryotic genome-maintenance functions. DNA Repair, 1(8), 579-600.

Hempel, J., \& Böhm, H. (1997). Betaxanthin pattern of hairy roots from Beta vulgaris var. lutea and its alteration by feeding of amino acids. Phytochemistry, 44(5), 847-852.

Herbach, K. M., Stintzing, F. C., \& Carle, R. (2005). Identification of heat-induced degradation products from purified betanin, phyllocactin and hylocerenin by high-performance liquid chromatography/electrospray ionization mass spectrometry. Rapid Communications in Mass Spectrometry, 19(18), 2603-2616.

Herbach, K. M., Stintzing, F. C., \& Carle, R. (2006). Betalain stability and degradation Structural and chromatic aspects. Journal of Food Science, 71(4), R41-R50.

Heuer, S., \& Strack, D. (1992). Synthesis of betanin from betanidin and UDP-glucose by a protein preparation from cell suspension cultures of Dorotheanthus bellidiformis (Burm. f.) NE Br. Planta, 186(4), 626-628.

Heuer, S., Vogt, T., Böhm, H., \& Strack, D. (1996). Partial purification and characterization of UDP-glucose: betanidin 5-O-and 6-O-glucosyltransferases from cell suspension cultures of Dorotheanthus bellidiformis (Burm. f.) $\mathrm{NE} \mathrm{Br}$. Planta, 199(2), 244-250.

Hichri, I., Barrieu, F., Bogs, J., Kappel, C., Delrot, S., \& Lauvergeat, V. (2011). Recent advances in the transcriptional regulation of the flavonoid biosynthetic pathway. Journal of Experimental Botany, 62(8), 2465-2483.

Hrazdina, G., Wagner, G. J., \& Siegelman, H. W. (1978). Subcellular localization of enzymes of anthocyanin biosynthesis in protoplasts. Phytochemistry, 17(1), 5356.

Hsieh, K., \& Huang, A. H. (2005). Lipid-rich tapetosomes in Brassica tapetum are composed of oleosin-coated oil droplets and vesicles, both assembled in and then detached from the endoplasmic reticulum. The Plant Journal, 43(6), 889899.

Hsieh, K., \& Huang, A. H. (2007). Tapetosomes in Brassica tapetum accumulate endoplasmic reticulum-derived flavonoids and alkanes for delivery to the pollen surface. The Plant Cell, 19(2), 582-596.

Hughes, N. M., Neufeld, H. S., \& Burkey, K. O. (2005). Functional role of anthocyanins in high-light winter leaves of the evergreen herb Galax urceolata. New Phytologist, 168(3), 575-587. 
Hvorup, R. N., Winnen, B., Chang, A. B., Jiang, Y., Zhou, X. F., \& Saier, M. H. (2003). The multidrug/oligosaccharidyl-lipid/polysaccharide (MOP) exporter superfamily. European Journal of Biochemistry, 270(5), 799-813.

Ibdah, M., Krins, A., Seidlitz, H. K., Heller, W., Strack, D., \& Vogt, T. (2002). Spectral dependence of flavonol and betacyanin accumulation in Mesembryanthemum crystallinum under enhanced ultraviolet radiation. Plant Cell \& Environment, 25(9), 1145-1154.

Inoue, H., Nojima, H., \& Okayama, H. (1990). High efficiency transformation of Escherichia coli with plasmids. Gene, 96(1), 23-28.

Isayenkova, J., Wray, V., Nimtz, M., Strack, D., \& Vogt, T. (2006). Cloning and functional characterisation of two regioselective flavonoid glucosyltransferases from Beta vulgaris. Phytochemistry, 67(15), 1598-1612.

Iwashina, T. (2001). Flavonoids and their distribution in plant families containing the betalain pigments. Annals of the Tsukuba Botanical Garden, 20, 11-74.

Iwashina, T., Ootani, S., \& Hayashi, K. (1988). On the pigmented spherical bodies and crystals in tepals of cactaceous species in reference to the nature of betalains or flavonols. Journal of Plant Research, 101(2), 175-184.

Jeong, S. T., Goto-Yamamoto, N., Kobayashi, S., \& Esaka, M. (2004). Effects of plant hormones and shading on the accumulation of anthocyanins and the expression of anthocyanin biosynthetic genes in grape berry skins. Plant Science, 167(2), 247-252.

Jin, H., Cominelli, E., Bailey, P., Parr, A., Mehrtens, F., Jones, J., Tonelli, C., Weisshaar, B., \& Martin, C. (2000). Transcriptional repression by AtMYB4 controls production of UV-protecting sunscreens in Arabidopsis. The EMBO Journal, 19(22), 6150-6161.

Joy, R. W., Sugiyama, M., Fukuda, H., \& Komamine, A. (1995). Cloning and characterization of polyphenol oxidase cDNAs of Phytolacca americana. Plant Physiology, 107(4), 1083-1089.

Kapadia, G. J., Tokuda, H., Konoshima, T., \& Nishino, H. (1996). Chemoprevention of lung and skin cancer by Beta vulgaris (beet) root extract. Cancer Letters, 100(12), 211-214.

Kay, R., Chan, A., Daly, M., \& McPherson, J. (1987). Duplication of CaMV $35 S$ promoter sequences creates a strong enhancer for plant genes. Science, 236(4806), 1299-1302.

Kenyon, W. H., \& Black, C. C. (1986). Electrophoretic analysis of protoplast, vacuole, and tonoplast vesicle proteins in Crassulacean acid metabolism plants. Plant Physiology, 82(4), 916-924.

Kimler, L., Mears, J., Mabry, T. J., \& Rösler, H. (1970). On the question of the mutual exclusiveness of betalains and anthocyanins. Taxon, 19(6), 875-878.

Kishima, Y., Shimaya, A., \& Adachi, T. (1995). Evidence that blue light induces betalain pigmentation in Portulaca callus. Plant Cell, Tissue and Organ Culture, 43(1), 67-70.

Kishima, Y., Suiko, M., \& Adachi, T. (1991). Betalain pigmentation in petal of Portulaca is preceded by a dramatic tyrosine accumulation. Journal of Plant Physiology, 137(4), 505-506.

Kitamura, S. (2006). Transport of flavonoids: from cytosolic synthesis to vacuolar accumulation. In E. Grotewold (Ed.), The Science of Flavonoids (pp. 123-146). New York: Springer 
Kitamura, S., Akita, Y., Ishizaka, H., Narumi, I., \& Tanaka, A. (2012). Molecular characterization of an anthocyanin-related glutathione $S$-transferase gene in cyclamen. Journal of Plant Physiology, 169(6), 636-642.

Kitamura, S., Matsuda, F., Tohge, T., Yonekura-Sakakibara, K., Yamazaki, M., Saito, K., \& Narumi, I. (2010). Metabolic profiling and cytological analysis of proanthocyanidins in immature seeds of Arabidopsis thaliana flavonoid accumulation mutants. The Plant Journal, 62(4), 549-559.

Kitamura, S., Shikazono, N., \& Tanaka, A. (2004). TRANSPARENT TESTA 19 is involved in the accumulation of both anthocyanins and proanthocyanidins in Arabidopsis. The Plant Journal, 37(1), 104-114.

Kleinboelting, N., Huep, G., Kloetgen, A., Viehoever, P., \& Weisshaar, B. (2012). GABI-Kat SimpleSearch: new features of the Arabidopsis thaliana T-DNA mutant database. Nucleic acids research, 40(D1), D1211-D1215.

Kobayashi, S., Ishimaru, M., Hiraoka, K., \& Honda, C. (2002). Myb-related genes of the Kyoho grape (Vitis labruscana) regulate anthocyanin biosynthesis. Planta, 215(6), 924-933.

Kochhar, V. K., Kochhar, S., \& Mohr, H. (1981). An analysis of the action of light on betalain synthesis in the seedling of Amaranthus caudatus, var. viridis. Planta, 151(1), 81-87.

Koes, R., Verweij, W., \& Quattrocchio, F. (2005). Flavonoids: a colorful model for the regulation and evolution of biochemical pathways. Trends in Plant Science, 10(5), 236-242.

Koiwa, H., Bressan, R. A., \& Hasegawa, P. M. (2006). Identification of plant stressresponsive determinants in Arabidopsis by large-scale forward genetic screens. Journal of Experimental Botany, 57(5), 1119-1128.

Koornneef, M. (1990). Mutations affecting the testa colour in Arabidopsis. Arabidopsis Information Service, 27, 1-4.

Kubasek, W. L., Shirley, B. W., McKillop, A., Goodman, H. M., Briggs, W., \& Ausubel, F. M. (1992). Regulation of flavonoid biosynthetic genes in germinating Arabidopsis seedlings. The Plant Cell, 4(10), 1229-1236.

Kugler, F., Stintzing, F. C., \& Carle, R. (2004). Identification of betalains from petioles of differently colored Swiss chard (Beta vulgaris L. ssp cicla [L.] Alef. Cv. Bright lights) by high-performance liquid chromatography-electrospray ionization mass spectrometry. Journal of Agricultural and Food Chemistry, 52(10), 2975-2981.

Kugler, F., Stintzing, F. C., \& Carle, R. (2007). Characterisation of betalain patterns of differently coloured inflorescences from Gomphrena globosa L. and Bougainvillea sp. by HPLC-DAD-ESI-MSn. Analytical and Bioanalytical Chemistry, 387(2), 637-648.

Kujala, T. S., Vienola, M. S., Klika, K. D., Loponen, J. M., \& Pihlaja, K. (2002). Betalain and phenolic compositions of four beetroot (Beta vulgaris) cultivars. European Food Research and Technology, 214(6), 505-510.

Kulich, I., Pecenkova, T., Sekeres, J., Smetana, O., Fendrych, M., Foissner, I., Hoftberger, M., \& Zarsky, V. (2013). Arabidopsis exocyst subcomplex containing subunit EXO70B1 is involved in autophagy-related transport to the vacuole. Traffic, 14(11), 1155-1165.

Kytridis, V. P., \& Manetas, Y. (2006). Mesophyll versus epidermal anthocyanins as potential in vivo antioxidants: evidence linking the putative antioxidant role to the proximity of oxy-radical source. Journal of Experimental Botany, 57(10), 2203-2210. 
Lange, C., Holtgräwe, D., Schulz, B., Weisshaar, B., \& Himmelbauer, H. (2008). Construction and characterization of a sugar beet (Beta vulgaris) fosmid library. Genome, 51(11), 948-951.

Leathers, R. R., Davin, C., \& Zrÿd, J. P. (1992). Betalain producing cell cultures of Beta vulgaris L. var. bikores monogerm (red beet). In Vitro - Plant, 28(2), 39-45.

Lee, D. W., \& Collins, T. M. (2001). Phylogenetic and ontogenetic influences on the distribution of anthocyanins and betacyanins in leaves of tropical plants. International Journal of Plant Sciences, 162(5), 1141-1153.

Leicht, B. G., \& Cheng, C. L. (2009). Reverse genetics: Identification of Arabidopsis lines with T-DNA insertions in a gene of interest (Vol. 30). Proceedings of the 30th Workshop/Conference of the Association for Biology Laboratory Education (ABLE).

Li, Y. G., Tanner, G., \& Larkin, P. (1996). The DMACA-HCl protocol and the threshold proanthocyanidin content for bloat safety in forage legumes. Journal of the Science of Food and Agriculture, 70(1), 89-101.

Li, Y. M., Qu, X. L., Li, X. L., Guo, L., \& Shen, X. (2008). Pigment distribution in fresh shoot tissues of four colored leaf Prunus taxa. ISHS Acta Horticulturae, 769, 399-405.

Lodge, D. J., Padamsee, M., Matheny, P. B., Aime, M. C., Cantrell, S. A., Boertmann, D., Kovalenko, A., Vizzini, A., Dentinger, B. T., \& Kirk, P. M. (2013). Molecular phylogeny, morphology, pigment chemistry and ecology in Hygrophoraceae (Agaricales). Fungal Diversity, 1-99.

Lu, Y.-P., Li, Z.-S., \& Rea, P. A. (1997). AtMRPl gene of Arabidopsis encodes a glutathione $S$-conjugate pump: isolation and functional definition of a plant ATP-binding cassette transporter gene. Proceedings of the National Academy of Sciences, 94(15), 8243-8248.

Luthje, S., \& Bottger, M. (1989). Hexabromoiridate IV as an electron acceptor: comparison with hexachloroiridate IV and hexacyanoferrate III. Biochimica et Biophysica Acta (BBA) - Bioenergetics, 977(3), 335-340.

Mabry, T. J., \& Dreiding, A. S. (1967). Chapter 4. The Betalains. In T. J. Mabry (Ed.), Recent Advances in Phytochemistry (pp. 145-160). New York: AppletonCentury-Crofts.

Mabry, T. J., Taylor, A., \& Turner, B. L. (1963). The betacyanins and their distribution. Phytochemistry, 2(1), 61-64.

Manetas, Y. (2006). Why some leaves are anthocyanic and why most anthocyanic leaves are red? Flora - Morphology, Distribution, Functional Ecology of Plants, 201(3), 163-177.

Marinova, K., Pourcel, L., Weder, B., Schwarz, M., Barron, D., Routaboul, J. M., Debeaujon, I., \& Klein, M. (2007). The Arabidopsis MATE transporter TT12 acts as a vacuolar flavonoid/ $\mathrm{H}^{+}$-antiporter active in proanthocyanidinaccumulating cells of the seed coat. The Plant Cell, 19(6), 2023-2038.

Marrs, K. A. (1996). The functions and regulation of glutathione $S$-transferases in plants. Annual Review of Plant Biology, 47(1), 127-158.

Marrs, K. A., Alfenito, M. R., Lloyd, A. M., \& Walbot, V. (1995). A glutathione Stransferase involved in vacuolar transfer encoded by the maize gene Bronze-2. Nature, 375(6530), 397-400.

Martin, C., Prescott, A., Mackay, S., Bartlett, J., \& Vrijlandt, E. (1991). Control of anthocyanin biosynthesis in flowers of Antirrhinum majus. The Plant Journal, 1(1), 37-49. 
Martin, S. S. (1977). Accumulation of the flavonoids betagarin and betavulgarin in Beta vulgaris infected by the fungus Cercospora beticola. Physiological Plant Pathology, 11(3), 297-303.

Martin, S. S. (1989). Analysis of constitutive and induced phenolics of Beta vulgaris by High Performance Liquid Chromatography. Journal of Sugar Beet Research, 26(2), 33-39.

Martinoia, E., Grill, E., Tommasini, R., Kreuz, K., \& Amrhein, N. (1993). ATPdependent glutathione $S$-conjugate export pump in the vacuolar membrane of plants. Nature, 364(6434), 247-249.

Marty, F. (1999). Plant Vacuoles. The Plant Cell, 11(4), 587-600.

Matile, P. (1978). Biochemistry and function of vacuoles. Annual Review of Plant Physiology, 29(1), 193-213.

Mayer, K., Schüller, C., Wambutt, R., Murphy, G., Volckaert, G., Pohl, T., Düsterhöft, A., Stiekema, W., Entian, K.-D., \& Terryn, N. (1999). Sequence and analysis of chromosome 4 of the plant Arabidopsis thaliana. Nature, 402(6763), 769-777.

McCormac, A., Wu, H., Bao, M., Wang, Y., Xu, R., Elliott, M., \& Chen, D.-F. (1998). The use of visual marker genes as cell-specific reporters of Agrobacteriummediated T-DNA delivery to wheat (Triticum aestivum L.) and barley (Hordeum vulgare L.). Euphytica, 99(1), 17-25.

McGrath, J. M., Shaw, R. S., Reyes, B., \& Weiland, J. (2004). Construction of a sugar beet BAC library from a hybrid with diverse traits. Plant Molecular Biology Reporter, 22(1), 23-28.

Mellway, R. D., Tran, L. T., Prouse, M. B., Campbell, M. M., \& Constabel, C. P. (2009). The wound-, pathogen-, and ultraviolet B-responsive MYB134 gene encodes an R2R3 MYB transcription factor that regulates proanthocyanidin synthesis in Poplar. Plant Physiology, 150(2), 924-941.

Merzlyak, M. N., \& Chivkunova, O. B. (2000). Light-stress-induced pigment changes and evidence for anthocyanin photoprotection in apples. Journal of Photochemistry and Photobiology, 55(2-3), 155-163.

Mol, J., Grotewold, E., \& Koes, R. (1998). How genes paint flowers and seeds. Trends in Plant Science, 3(6), 212-217.

Moriyasu, Y., \& Hillmer, S. (2000). Autophagy and vacuole formation. Vacuolar Compartments, 5,71 .

Mosco, A. (2012). Tissue localization of betacyanins in cactus stems. Revista Mexicana De Biodiversidad, 83(2), 413-420.

Mueller, L. A., Goodman, C. D., Silady, R. A., \& Walbot, V. (2000). AN9, a petunia glutathione $S$-transferase required for anthocyanin sequestration, is a flavonoidbinding protein. Plant Physiology, 123(4), 1561-1570.

Mueller, L. A., Hinz, U., Uze, M., Sautter, C., \& Zrÿd, J. P. (1997a). Biochemical complementation of the betalain biosynthetic pathway in Portulaca grandiflora by a fungal 3,4-dihydroxyphenylalanine dioxygenase. Planta, 203(2), 260-263.

Mueller, L. A., Hinz, U., \& Zrÿd, J.-P. (1996). Characterization of a tyrosinase from Amanita muscaria involved in betalain biosynthesis. Phytochemistry, 42(6), $1511-1515$

Mueller, L. A., Hinz, U., \& Zrÿd, J. P. (1997b). The formation of betalamic acid and muscaflavin by recombinant dopa-dioxygenase from Amanita. Phytochemistry, 44(4), 567-569.

Mukundan, U., Bhide, V., \& Dawda, H. (1999). Production of betalains by hairy root cultures of Beta Vulgaris L. In T.-J. Fu, G. Singh \& W. Curtis (Eds.), Plant Cell 
and Tissue Culture for the Production of Food Ingredients (pp. 121-127): Springer US.

Munns, R. (2002). Comparative physiology of salt and water stress. Plant Cell \& Environment, 25(2), 239-250.

Murashige, T., \& Skoog, F. (1962). A Revised Medium for Rapid Growth and Bio Assays with Tobacco Tissue Cultures. Physiologia Plantarum, 15(3), 473-497.

Musso, H. (1979). The pigments of fly agaric, Amanita muscaria. Tetrahedron, 35(24), 2843-2853.

Nakashima, T., Araki, T., \& Ueno, O. (2011). Photoprotective function of betacyanin in leaves of Amaranthus cruentus L. under water stress. Photosynthetica, 49(4), 497-506.

Nakatsuka, T., Yamada, E., Takahashi, H., Imamura, T., Suzuki, M., Ozeki, Y., Tsujimura, I., Saito, M., Sakamoto, Y., Sasaki, N., \& Nishihara, M. (2013). Genetic engineering of yellow betalain pigments beyond the species barrier. Scientific Reports, 3, 1970.

Neill, S., \& Gould, K. S. (2000). Optical properties of leaves in relation to anthocyanin concentration and distribution. Canadian Journal of Botany, 77(12), 1777-1782.

Neill, S. O., Gould, K. S., Kilmartin, P. A., Mitchell, K. A., \& Markham, K. R. (2002). Antioxidant activities of red versus green leaves in Elatostema rugosum. Plant Cell \& Environment, 25(4), 539-547.

Nemzer, B., Pietrzkowski, Z., Sporna, A., Stalica, P., Thresher, W., Michalowski, T., \& Wybraniec, S. (2011). Betalainic and nutritional profiles of pigment-enriched red beet root (Beta vulgaris L.) dried extracts. Food Chemistry, 127(1), 42-53.

Nielson, L. R., \& Harley, S. M. (1996). Chemotaxonomy: simple tests for distinguishing between anthocyanins and betacyanins. Journal of Biological Education, 30(2), 88-90.

Nozzolillo, C. (1973). A survey of anthocyanin pigments in seedling legumes. Canadian Journal of Botany, 51(5), 911-915.

Nozzolillo, C., \& McNeill, J. (1985). Anthocyanin pigmentation in seedlings of selected species of Phaseolus and Vigna (Fabaceae). Canadian Journal of Botany, 63(6), 1066-1071.

O'Malley, R. C., Alonso, J. M., Kim, C. J., Leisse, T. J., \& Ecker, J. R. (2007). An adapter ligation-mediated PCR method for high-throughput mapping of T-DNA inserts in the Arabidopsis genome. Nature protocols, 2(11), 2910-2917.

Page, J. E., \& Towers, G. H. (2002). Anthocyanins protect light-sensitive thiarubrine phototoxins. Planta, 215(3), 478-484.

Peters, D. J., \& Constabel, C. P. (2002). Molecular analysis of herbivore-induced condensed tannin synthesis: cloning and expression of dihydroflavonol reductase from trembling aspen (Populus tremuloides). The Plant Journal, 32(5), 701-712.

Petroni, K., \& Tonelli, C. (2011). Recent advances on the regulation of anthocyanin synthesis in reproductive organs. Plant Science, 181(3), 219-229.

Petrus-Vancea, A., Cachita-Cosma, D., Pop, L., \& Purcarea, C. (2010). Histoanatomical specific features of red beet and sugar beet vitroplantlets. Analele Universităţii din Oradea, Fascicula: Protecţia Mediului, 15, 740-749.

Pfaffl, M. W. (2001). A new mathematical model for relative quantification in real-time RT-PCR. Nucleic Acids Research, 29(9), e45-e45.

Pfaffl, M. W., Tichopad, A., Prgomet, C., \& Neuvians, T. P. (2004). Determination of stable housekeeping genes, differentially regulated target genes and sample 
integrity: BestKeeper - Excel-based tool using pair-wise correlations. Biotechnology Letters, 26(6), 509-515.

Piattelli, M., Giudici De Nicola, M., \& Castrogiovanni, V. (1969). Photocontrol of amaranthin synthesis in Amaranthus tricolor. Phytochemistry, 8(4), 731-736.

Pourcel, L., Bohorquez-Restrepo, A., Irani, N. G., \& Grotewold, E. (2012). Anthocyanin biosynthesis, regulation, and transport: new insights from model species. Recent Advances in Polyphenol Research, 3, 143-161.

Poustka, F., Irani, N. G., Feller, A., Lu, Y., Pourcel, L., Frame, K., \& Grotewold, E. (2007). A trafficking pathway for anthocyanins overlaps with the endoplasmic reticulum-to-vacuole protein-sorting route in Arabidopsis and contributes to the formation of vacuolar inclusions. Plant Physiology, 145(4), 1323-1335.

Powell, A. M., \& Weedin, J. F. (2004). Cacti of the Trans-Pecos \& Adjacent Areas: Texas Tech University Press.

Pyo, Y.-H., Lee, T.-C., Logendra, L., \& Rosen, R. T. (2004). Antioxidant activity and phenolic compounds of Swiss chard (Beta vulgaris subspecies cycla) extracts. Food Chemistry, 85(1), 19-26.

Reyes, L. F., \& Cisneros-Zevallos, L. (2003). Wounding stress increases the phenolic content and antioxidant capacity of purple-flesh potatoes (Solanum tuberosum L.). Journal of Agricultural and Food Chemistry, 51(18), 5296-5300.

Richard, S., Lapointe, G., Rutledge, R. G., \& Séguin, A. (2000). Induction of chalcone synthase expression in white spruce by wounding and jasmonate. Plant and Cell Physiology, 41(8), 982-987.

Richardson, P. M. (1981). Phytoalexin induction in Beta and Spinacia. Biochemical Systematics and Ecology, 9(2-3), 105-107.

Rieu, I., \& Powers, S. J. (2009). Real-time quantitative RT-PCR: design, calculations, and statistics. The Plant Cell, 21(4), 1031-1033.

Robinson, D. G., Galili, G., Herman, E., \& Hillmer, S. (1998). Topical aspects of vacuolar protein transport: autophagy and prevacuolar compartments. Journal of Experimental Botany, 49(325), 1263-1270.

Rosado, A., Hicks, Glenn R., Norambuena, L., Rogachev, I., Meir, S., Pourcel, L., Zouhar, J., Brown, Michelle Q., Boirsdore, Marietta P., Puckrin, Rachel S., Cutler, Sean R., Rojo, E., Aharoni, A., \& Raikhel, Natasha V. (2011). Sortin1Hypersensitive mutants link vacuolar-trafficking defects and flavonoid metabolism in Arabidopsis vegetative tissues. Chemistry \& Biology, 18(2), 187197.

Rowan, D. D., Cao, M., Lin-Wang, K., Cooney, J. M., Jensen, D. J., Austin, P. T., Hunt, M. B., Norling, C., Hellens, R. P., Schaffer, R. J., \& Allan, A. C. (2009). Environmental regulation of leaf colour in red 35S:PAP1 Arabidopsis thaliana. New Phytologist, 182(1), 102-115.

Sasaki, N., Abe, Y., Goda, Y., Adachi, T., Kasahara, K., \& Ozeki, Y. (2009). Detection of DOPA 4,5-dioxygenase (DOD) activity using recombinant protein prepared from Escherichia coli cells harboring cDNA encoding DOD from Mirabilis jalapa. Plant \& Cell Physiology, 50(5), 1012-1016.

Sasaki, N., Abe, Y., Wada, K., Koda, T., Goda, Y., Adachi, T., \& Ozeki, Y. (2005a). Amaranthin in feather cockscombs is synthesized via glucuronylation at the cyclo-DOPA glucoside step in the betacyanin biosynthetic pathway. Journal of Plant Research, 118(6), 439-442.

Sasaki, N., Adachi, T., Koda, T., \& Ozeki, Y. (2004). Detection of UDP-glucose: cycloDOPA 5-O-glucosyltransferase activity in four o'clocks (Mirabilis jalapa L.). FEBS letters, 568(1), 159-162. 
Sasaki, N., Wada, K., Koda, T., Kasahara, K., Adachi, T., \& Ozeki, Y. (2005b). Isolation and characterization of cDNAs encoding an enzyme with glucosyltransferase activity for cyclo-DOPA from four o'clocks and feather cockscombs. Plant \& Cell Physiology, 46(4), 666-670.

Schliemann, W., Kobayashi, N., \& Strack, D. (1999). The decisive step in betaxanthin biosynthesis is a spontaneous reaction. Plant Physiology, 119(4), 1217-1232.

Schliemann, W., \& Strack, D. (1998). Intramolecular stabilization of acylated betacyanins. Phytochemistry, 49(2), 585-588.

Schroeder, J. I., Allen, G. J., Hugouvieux, V., Kwak, J. M., \& Waner, D. (2001). Guard cell signal transduction. Annual Review of Plant Physiology and Plant Molecular Biology, 52(1), 627-658.

Sciuto, S., Oriente, G., \& Piattelli, M. (1972). Betanidin glucosylation in Opuntia dillenii. Phytochemistry, 11(7), 2259-2262.

Sekiguchi, H., Ozeki, Y., \& Sasaki, N. (2010). In vitro synthesis of betaxanthins using recombinant DOPA 4,5-dioxygenase and evaluation of their radical-scavenging activities. Journal of Agricultural and Food Chemistry.

Sepulveda-Jimenez, G., Rueda-Benitez, P., Porta, H., \& Rocha-Sosa, M. (2005). A red beet (Beta vulgaris) UDP-glucosyltransferase gene induced by wounding, bacterial infiltration and oxidative stress. Journal of Experimental Botany, 56(412), 605-611.

Sepúlveda-Jiménez, G., Rueda-Benítez, P., Porta, H., \& Rocha-Sosa, M. (2004). Betacyanin synthesis in red beet (Beta vulgaris) leaves induced by wounding and bacterial infiltration is preceded by an oxidative burst. Physiological \& Molecular Plant Pathology, 64(3), 125-133.

Shang, Y., Schwinn, K. E., Bennett, M. J., Hunter, D. A., Waugh, T. L., Pathirana, N. N., Brummell, D. A., Jameson, P. E., \& Davies, K. M. (2007). Methods for transient assay of gene function in floral tissues. Plant Methods, 3(1), 1.

Shang, Y., Venail, J., Mackay, S., Bailey, P. C., Schwinn, K. E., Jameson, P. E., Martin, C. R., \& Davies, K. M. (2011). The molecular basis for venation patterning of pigmentation and its effect on pollinator attraction in flowers of Antirrhinum. New Phytologist, 189(2), 602-615.

Shi, H., Ishitani, M., Kim, C., \& Zhu, J. K. (2000). The Arabidopsis thaliana salt tolerance gene $S O S 1$ encodes a putative $\mathrm{Na}^{+} / \mathrm{H}^{+}$antiporter. Prococeedings of the National Academy of Sciences, 97(12), 6896-6901.

Shi, H., Lee, B.-h., Wu, S.-J., \& Zhu, J.-K. (2002). Overexpression of a plasma membrane $\mathrm{Na}^{+} / \mathrm{H}^{+}$antiporter gene improves salt tolerance in Arabidopsis thaliana. Nature Biotechnology, 21(1), 81-85.

Shimada, S., Otsuki, H., \& Sakuta, M. (2007). Transcriptional control of anthocyanin biosynthetic genes in the Caryophyllales. Journal of Experimental Botany, 58(5), 957-967.

Shin, D. H., Choi, M., Kim, K., Bang, G., Cho, M., Choi, S.-B., Choi, G., \& Park, Y.-I. (2013). HY5 regulates anthocyanin biosynthesis by inducing the transcriptional activation of the MYB75/PAP1 transcription factor in Arabidopsis. FEBS Letters, 587(10), 1543-1547.

Singh, K., Rani, A., Kumar, S., Sood, P., Mahajan, M., Yadav, S. K., Singh, B., \& Ahuja, P. S. (2008). An early gene of the flavonoid pathway, flavanone 3hydroxylase, exhibits a positive relationship with the concentration of catechins in tea (Camellia sinensis). Tree Physiology, 28(9), 1349-1356.

Sloan, D. B., Keller, S. R., Berardi, A. E., Sanderson, B. J., Karpovich, J. F., \& Taylor, D. R. (2012). De novo transcriptome assembly and polymorphism detection in 
the flowering plant Silene vulgaris (Caryophyllaceae). Molecular Ecology Resources, 12(2), 333-343.

Small, C. J., \& Pecket, R. C. (1982). The ultrastructure of anthocyanoplasts in redcabbage. Planta, 154(2), 97-99.

Solangaarachchi, S. M., \& Gould, K. S. (2001). Anthocyanin pigmentation in the adventitious roots of Metrosideros excelsa (Myrtaceae). New Zealand Journal of Botany, 39(1), 161-166.

Solovchenko, A. E., \& Merzlyak, M. N. (2008). Screening of visible and UV radiation as a photoprotective mechanism in plants. Russian Journal of Plant Physiology, 55(6), 719-737.

Staehelin, L. A. (1997). The plant ER: a dynamic organelle composed of a large number of discrete functional domains. The Plant Journal, 11(6), 1151-1165.

Stafford, H. A. (1994). Anthocyanins and betalains - evolution of the mutually exclusive pathways. Plant Science, 101(2), 91-98.

Steglich, W., \& Strack, D. (1990). Betalains. In B. Arnold (Ed.), The Alkaloids: Chemistry and Pharmacology (Vol. Volume 39, pp. 1-62). London: Academic Press.

Steiner, U., Schliemann, W., Bohm, H., \& Strack, D. (1999). Tyrosinase involved in betalain biosynthesis of higher plants. Planta, 208(1), 114-124.

Steyn, W. J., Wand, S. J. E., Holcroft, D. M., \& Jacobs, G. (2002). Anthocyanins in vegetative tissues: a proposed unified function in photoprotection. New Phytologist, 155(3), 349-361.

Stiles, E. A., Cech, N. B., Dee, S. M., \& Lacey, E. P. (2007). Temperature-sensitive anthocyanin production in flowers of Plantago lanceolata. Physiologia Plantarum, 129(4), 756-765.

Stintzing, F. C., \& Carle, R. (2004). Functional properties of anthocyanins and betalains in plants, food, and in human nutrition. Trends in Food Science \& Technology, 15(1), 19-38.

Stintzing, F. C., \& Carle, R. (2007). Betalains - emerging prospects for food scientists. Trends in Food Science \& Technology, 18(10), 514-525.

Stintzing, F. C., Herbach, K. M., Mosshammer, M. R., Carle, R., Yi, W., Sellappan, S., Akoh, C. C., Bunch, R., \& Felker, P. (2005). Color, betalain pattern, and antioxidant properties of cactus pear (Opuntia spp.) clones. Journal of Agricultural and Food Chemistry, 53(2), 442-451.

Strack, D., Bokern, M., Marxen, N., \& Wray, V. (1988). Feruloylbetanin from petals of Lampranthus and feruloylamaranthin from cell suspension cultures of Chenopodium rubrum. Phytochemistry, 27(11), 3529-3531.

Strack, D., Vogt, T., \& Schliemann, W. (2003). Recent advances in betalain research. Phytochemistry, 62(3), 247-269.

Sun, Y., Li, H., \& Huang, J. R. (2012). Arabidopsis TT19 functions as a carrier to transport anthocyanin from the cytosol to tonoplasts. Molecular Plant, 5(2), 387400.

Suresh, B., Thimmaraju, R., Bhagyalakshmi, N., \& Ravishankar, G. (2004). Polyamine and methyl jasmonate-influenced enhancement of betalaine production in hairy root cultures of Beta vulgaris grown in a bubble column reactor and studies on efflux of pigments. Process Biochemistry, 39(12), 2091-2096.

Svenson, J., Smallfield, B. M., Joyce, N. I., Sanson, C. E., \& Perry, N. B. (2008). Betalains in red and yellow varieties of the Andean tuber crop ulluco (Ullucus tuberosus). Journal of Agricultural and Food Chemistry, 56(17), 7730-7737. 
Taiz, L. (1992). The Plant Vacuole. The Journal of Experimental Biology, 172(Pt 1), 113-122.

Takahashi, K., Takamura, E., \& Sakuta, M. (2009). Isolation and expression analysis of two DOPA dioxygenases in Phytolacca americana. Zeitschrift für Naturforschung. C, a Journal of Biosciences, 64(7), 564.

Tamagnone, L., Merida, A., Parr, A., Mackay, S., Culianez-Macia, F. A., Roberts, K., \& Martin, C. (1998). The AmMYB308 and AmMYB330 transcription factors from Antirrhinum regulate phenylpropanoid and lignin biosynthesis in transgenic tobacco. The Plant Cell, 10(2), 135-154.

Tamura, K., Peterson, D., Peterson, N., Stecher, G., Nei, M., \& Kumar, S. (2011). MEGA5: molecular evolutionary genetics analysis using maximum likelihood, evolutionary distance, and maximum parsimony methods. Molecular Biology and Evolution, 28(10), 2731-2739.

Tanaka, Y., Sasaki, N., \& Ohmiya, A. (2008). Biosynthesis of plant pigments: anthocyanins, betalains and carotenoids. The Plant Journal, 54(4), 733-749.

Terradas, F., \& Wyler, H. (1991). The secodopas, natural pigments in Hygrocybe conica and Amanita muscaria. Phytochemistry, 30(10), 3251-3253.

Tesoriere, L., Allegra, M., Butera, D., \& Livrea, M. A. (2004). Absorption, excretion, and distribution of dietary antioxidant betalains in LDLs: potential health effects of betalains in humans. The American Journal of Clinical Nutrition, 80(4), 941945.

Thakur, M., \& Nozzolillo, C. (1978). Anthocyanin pigmentation in roots of Impatiens species. Canadian Journal of Botany, 56(22), 2898-2903.

Theodoulou, F. L. (2000). Plant ABC transporters. Biochimica et Biophysica Acta, 1465(1-2), 79-103.

Thompson, E. P., Davies, J. M., \& Glover, B. J. (2010). Identifying the transporters of different flavonoids in plants. Plant Signaling \& Behavior, 5(7), 860-863.

Trezzini, G., \& Zrÿd, J.-P. (1989). Portulaca grandiflora: a model system for the study of the biochemistry and genetics of betalain synthesis. Paper presented at the International Symposium on In Vitro Culture and Horticultural Breeding 280.

Trezzini, G. F., \& Zyrd, J. P. (1990). Portulaca grandiflora: a model system for the study of the biochemistry and genetics of betalain synthesis. Acta Horticulturae, 280, 581-585.

Troyer, J. R. (1964). Anthocyanin formation in excised segments of buckwheat-seedling hypocotyls. Plant Physiology, 39(6), 907.

Vain, P., Keen, N., Murillo, J., Rathus, C., Nemes, C., \& Finer, J. J. (1993). Development of the particle inflow gun. Plant Cell, Tissue and Organ Culture, 33(3), 237-246.

Verchot-Lubicz, J., \& Goldstein, R. E. (2010). Cytoplasmic streaming enables the distribution of molecules and vesicles in large plant cells. Protoplasma, 240(14), 99-107.

Vincent, M. (1994). Development and characterisation of an inducible system of betalain synthesis in cell cultures of beetroot (Beta vulgaris). Dublin City University.

Vogt, T., Ibdah, M., Schmidt, J., Wray, V., Nimtz, M., \& Strack, D. (1999). Lightinduced betacyanin and flavonol accumulation in bladder cells of Mesembryanthemum crystallinum. Phytochemistry, 52(4), 583-592.

Vogt, T., \& Jones, P. (2000). Glycosyltransferases in plant natural product synthesis: characterization of a supergene family. Trends in Plant Science, 5(9), 380-386. 
Vogt, T., Zimmermann, E., Grimm, R., Meyer, M., \& Strack, D. (1997). Are the characteristics of betanidin glucosyltransferases from cell-suspension cultures of Dorotheanthus bellidiformis indicative of their phylogenetic relationship with flavonoid glucosyltransferases? Planta, 203(3), 349-361.

Wang, C.-Q., Song, H., Gong, X.-Z., Hu, Q.-G., Liu, F., \& Wang, B.-S. (2007). Correlation of tyrosinase activity and betacyanin biosynthesis induced by dark in C3 halophyte Suaeda salsa seedlings. Plant Science, 173(5), 487-494.

Wang, C.-Q., Xu, C., Wei, J.-G., Wang, H.-B., \& Wang, S.-H. (2008). Enhanced tonoplast $\mathrm{H}^{+}$-ATPase activity and superoxide dismutase activity in the halophyte Suaeda salsa containing high level of betacyanin. Journal of Plant Growth Regulation, 27(1), 58-67.

Wang, H., Cao, G., \& Prior, R. L. (1997). Oxygen radical absorbing capacity of anthocyanins. Journal of Agricultural and Food Chemistry, 45(2), 304-309.

Wang, Y., Zhou, B., Sun, M., Li, Y., \& Kawabata, S. (2012). UV-A light induces anthocyanin biosynthesis in a manner distinct from synergistic blue + UV-B light and UV-A/Blue light responses in different parts of the hypocotyls in turnip seedlings. Plant \& Cell Physiology, 53(8), 1470-1480.

Winder, A. J., \& Harris, H. (1991). New assays for the tyrosine hydroxylase and DOPA oxidase activities of tyrosinase. European Journal of Biochemistry, 198(2), 317326.

Winkel-Shirley, B. (2001). Flavonoid biosynthesis. A colorful model for genetics, biochemistry, cell biology, and biotechnology. Plant Physiology, 126(2), 485493.

Winkel-Shirley, B. (1999). Evidence for enzyme complexes in the phenylpropanoid and flavonoid pathways. Physiologia Plantarum, 107(1), 142-149.

Wolyn, D., \& Gabelman, W. (1989). Inheritance of root and petiole pigmentation in red table beet. Journal of Heredity, 80(1), 33-38.

Wybraniec, S. (2005). Formation of decarboxylated betacyanins in heated purified betacyanin fractions from red beet root (Beta vulgaris L.) monitored by LC-MS/MS. Journal of Agricultural and Food Chemistry, 53(9), 3483-3487.

Wybraniec, S., Nowak-Wydra, B., \& Mizrahi, Y. (2006). ${ }^{1} \mathrm{H}$ and ${ }^{13} \mathrm{C}$ NMR spectroscopic structural elucidation of new decarboxylated betacyanins. Tetrahedron Letters, 47(11), 1725-1728.

Wyler, H., Meuer, U., Bauer, J., \& Stravs-Mombelli, L. (1984). Cyclodopa glucoside $(=(2 S)-5-(\beta$-D-glocopyranosyloxy)-6-hydroxyindoline-2-carboxylic acid) and its occurrence in red beet (Beta vulgaris var. rubra L.). Helvetica Chimica Acta, 67(5), 1348-1355.

Xie, D.-Y., \& Dixon, R. A. (2005). Proanthocyanidin biosynthesis - still more questions than answers? Phytochemistry, 66(18), 2127-2144.

Yamagishi, M., Shimoyamada, Y., Nakatsuka, T., \& Masuda, K. (2010). Two R2R3$M Y B$ genes, homologs of petunia AN2, regulate anthocyanin biosyntheses in flower tepals, tepal spots and leaves of asiatic hybrid lily. Plant \& Cell Physiology, 51(3), 463-474.

Yamamoto, K., Kobayashi, N., Yoshitama, K., Teramoto, S., \& Komamine, A. (2001). Isolation and purification of tyrosine hydroxylase from callus cultures of Portulaca grandiflora. Plant \& Cell Physiology, 42(9), 969-975.

Yamazaki, M., Nakajima, J.-I., Yamanashi, M., Sugiyama, M., Makita, Y., Springob, K., Awazuhara, M., \& Saito, K. (2003). Metabolomics and differential gene expression in anthocyanin chemo-varietal forms of Perilla frutescens. Phytochemistry, 62(6), 987-995. 
Yazaki, K. (2005). Transporters of secondary metabolites. Current Opinion in Plant Biology, 8(3), 301-307.

Yokoi, S., Quintero, F. J., Cubero, B., Ruiz, M. T., Bressan, R. A., Hasegawa, P. M., \& Pardo, J. M. (2002). Differential expression and function of Arabidopsis thaliana $\mathrm{NHX} \mathrm{Na} / \mathrm{H}^{+}$antiporters in the salt stress response. The Plant Journal, 30(5), 529-539.

Young, T. E., Griswold, J. R., \& Hulbert, M. H. (1974). Melanin. I. Kinetics of the oxidative cyclization of dopa to dopaquinone. The Journal of Organic Chemistry, 39(13), 1980-1982.

Zhang, H., Wang, L., Deroles, S., Bennett, R., \& Davies, K. (2006). New insight into the structures and formation of anthocyanic vacuolar inclusions in flower petals. BMC Plant Biology, 6(1), 29.

Zhao, J., \& Dixon, R. A. (2010). The 'ins' and 'outs' of flavonoid transport. Trends in Plant Science, 15(2), 72-80.

Zhao, S.-Z., Sun, H.-Z., Gao, Y., Sui, N., \& Wang, B.-S. (2011). Growth regulatorinduced betacyanin accumulation and dopa-4,5-dioxygenase (DODA) gene expression in euhalophyte Suaeda salsa calli. In Vitro Cellular \& Developmental Biology - Plant, 47(3), 391-398.

Zrÿd, J., Bauer, J., Wyler, H., \& Lavanchy, P. (1982). Pigment biosynthesis and precursor metabolism in red beet semi-continuous cell suspension cultures. Paper presented at the Plant tissue culture 1982: proceedings, 5th International Congress of Plant Tissue and Cell Culture held at Tokyo and Lake Yamanake, Japan, July 11-16, 1982/edited by Akio Fujiwara.

Zrÿd, J. P., \& Christinet, L. (2004). Betalains. In K. M. Davies (Ed.), Plant pigments and their manipulation (pp. 185-213). Victoria, Australia: Blackwell Publishing LTD.

Zuluaga, D. L., Gonzali, S., Loreti, E., Pucciariello, C., Degl'Innocenti, E. D., Guidi, L., Alpi, A., \& Perata, P. (2008). Arabidopsis thaliana MYB75/PAP1 transcription factor induces anthocyanin production in transgenic tomato plants. Functional Plant Biology, 35(7), 606-618. 


\section{Appendix 1: Media, buffers and solutions}

\subsection{Culture media and solutions}

\subsubsection{Luria-Bertani (LB) broth and media}

Dissolve $10 \mathrm{~g} \mathrm{~L}^{-1}$ bactotryptone, $5 \mathrm{~g} \mathrm{~L}^{-1}$ sodium chloride dissolved in $1 \mathrm{~L}$ water and autoclave. For LB-agar, add $1.5 \%$ (w/v) agar.

\subsubsection{Hydroponic solution}

Hydroponic solution contains $2.25 \mathrm{~g} \mathrm{~L}^{-1} \mathrm{MS}$ salts (Murashige \& Skoog, 1962) and $10 \mathrm{~g} \mathrm{~L}^{-1}$ sucrose dissolved in water. Adjust solution to $\mathrm{pH} 6$ with $\mathrm{KOH}$ and autoclave.

\subsubsection{Media \#2}

Add $7 \mathrm{~g} \mathrm{~L}^{-1}$ agar to the hydroponic solution (Appendix 1.1.2) before autoclaving.

\subsection{Agrobacterium transfection solution}

To $250 \mathrm{~mL}$ of autoclaved $5 \%(\mathrm{w} / \mathrm{v})$ sucrose, add $0.02 \%(\mathrm{v} / \mathrm{v})$ Pulse detergent.

\subsection{Antibiotics}

Dissolve antibiotic salts Spectinomycin (spectinomycin dihydrochloride), Ampicillin (sodium salt of ampicillin) and Streptomycin (streptomycin sulphate) in water to a concentration of $100 \mathrm{mg} \mathrm{mL}^{-1}$ stocks. Filter sterilise antibiotic solutions through $0.2 \mu \mathrm{m}$ membranes and store at $4{ }^{\circ} \mathrm{C}$, except for Ampicillin which is stored at $-20{ }^{\circ} \mathrm{C}$.

\subsection{Electrophoresis gels and reagents}

\subsubsection{TBE buffer and gel}

$10 \times$ TBE buffer contains $0.89 \mathrm{M}$ tris-base, $0.89 \mathrm{M}$ boric acid and $20 \mathrm{mM}$ ethylenediaminetetraacetic acid (EDTA), made up with water and is autoclaved. For DNA electrophoresis, dilute $10 \times$ TBE buffer tenfold with water. To make TBE gels, add $1-2 \%(\mathrm{w} / \mathrm{v})$ agarose to $10 \mathrm{x}$ TBE buffer before autoclaving. Before making gel for electrophoresis, add $2.5 \mu \mathrm{L}$ of ethidium bromide per $100 \mathrm{~mL}$. 


\subsubsection{MOPS Buffer and MOPS gel with formaldehyde}

$10 \times$ MOPS buffer contains 0.2 M 3-(N-morpholino)propanesulfonic acid (MOPS), $50 \mathrm{mM} \mathrm{NaOAc}, 10 \mathrm{mM}$ EDTA and made up with water and is adjusted to $\mathrm{pH} 7.0$ with $\mathrm{NaOH}$. Autoclave solution and store at room temperature. For RNA electrophoresis, dilute $10 \times$ MOPS buffer tenfold with water. To make a $50 \mathrm{~mL}$ MOPS gel with formaldehyde, dissolve $0.6 \mathrm{~g}$ agarose into $42.5 \mathrm{~mL}$ water, and then add $5 \mathrm{~mL}$ of $10 \mathrm{x}$ MOPS buffer and $2.5 \mathrm{~mL}$ formaldehyde.

\subsubsection{0 x DNA loading dye}

10 x DNA loading dye contains $20 \%$ (w/v) Ficoll 400, $1 \%(w / v)$ SDS, 0.1 M EDTA ( $\mathrm{pH} 8.0), 0.25 \%(\mathrm{w} / \mathrm{v})$ xylene cyanol and $0.25 \%(\mathrm{w} / \mathrm{v})$ bromophenol blue, in water. Dye is stored at room temperature.

\subsubsection{0 x RNA loading dye}

To make $10 \mathrm{~mL}$ of $10 \times$ RNA loading dye, add $0.2 \mathrm{~mL}$ of 0.5 M EDTA (pH 8.0), $9.8 \mathrm{~mL}$ of formamide, $2.5 \mathrm{mg}$ of xylene cyanol and $2.5 \mathrm{mg}$ of bromophenol blue. Alliquot dye into $1.5 \mathrm{~mL}$ microcentrifuge tubes, store at $-20^{\circ} \mathrm{C}$.

For loading RNA into MOPS gel (Appendix 1.4.2), to $2 \mu \mathrm{L}$ of each sample add $10 \mu \mathrm{L}$ of sodium diformamide, $3.5 \mu \mathrm{L}$ of formaldehyde, $2 \mu \mathrm{L}$ of $10 \times$ RNA loading dye, $2 \mu \mathrm{L}$ of $10 \mathrm{x}$ MOPS buffer (Appendix 1.4.2) and $0.5 \mu \mathrm{L}$ of ethidium bromide.

\subsection{Nucleic acid extraction buffers and reagents}

\subsection{1 gDNA extraction buffer}

gDNA extraction buffer contains $200 \mathrm{mM}$ Tris $\mathrm{HCl}(\mathrm{pH}$ 7.5), $250 \mathrm{mM} \mathrm{NaCl}, 25 \mathrm{mM}$ EDTA, $0.5 \%(\mathrm{w} / \mathrm{v}) \mathrm{SDS}$, then store buffer at room temperature. Before use, add $10 \mathrm{mM}$ DTT.

\subsubsection{TE buffer}

TE buffer contains $10 \mathrm{mM}$ Tris $\mathrm{HCl}(\mathrm{pH} 7.5)$ and $1 \mathrm{mM}$ EDTA. Store the TE buffer at room temperature. 


\subsection{Pigment analyses}

\subsubsection{Betalain XT buffer}

Betalain XT buffer contains $100 \%$ Methanol: $0.1 \mathrm{M}$ ascorbic acid (80:20). Buffer is to be made fresh before pigment extraction.

\subsubsection{McIlvaine buffer system}

The McIlvaine buffer system is adjusted with citrate-phosphate to $\mathrm{pH} 6.0$ for betalain stability. Dissolve $0.2 \mathrm{M}$ disodium hydrogen $\left(\mathrm{Na}_{2} \mathrm{PH}\right)$, and $0.1 \mathrm{M}$ citric acid in water. Spectrophotometric measurements use $900-980 \mu \mathrm{L}$ of McIlvaine with $20-100 \mu \mathrm{L}$ of pigment extract. The buffer is to be made fresh before measurements.

\subsection{Miscellaneous solutions}

\subsubsection{L-DOPA and cyclo-DOPA solutions}

Powdered forms of L-3,4-dihydroxyphenylalanine (L-DOPA) and cyclo-dihydroxyphenylalanine (cyclo-DOPA) are dissolved into $10 \mathrm{mM}$ stock solutions with water. These stocks are used fresh or stored frozen in $-20{ }^{\circ} \mathrm{C}$ until needed. As both L-DOPA and cyclo-DOPA compounds are not stable in liquid form, the antioxidant ascorbic acid can be added to the solution at $20 \mathrm{mM}$ concentration.

Stocks of cyclo-DOPA was synthesised by Lesley Larsen (Plant \& Food Research, Lincoln) to approximately $90 \%$ purity. The other $10 \%$ was made up of inorganic salts (potassium hexacyanoferrate). These inorganic salts are unable permeate the plasma membrane of root cells (Luthje \& Bottger, 1989). The concentration of cyclo-DOPA in these stocks was taken into account when making up $10 \mathrm{mM}$ stocks with water.

\subsubsection{IPTG and X-gal}

Dissolve chemicals Isopropyl $\beta$-D-1-thiogalactopyranoside (IPTG) and 5-bromo-4-chloro-3-indolyl- $\beta$-D-galactopyranoside (X-gal) in water to $200 \mathrm{mg} \mathrm{mL}^{-1}$ and $20 \mathrm{mg} \mathrm{mL}^{-1}$ stocks, respectively. Store in $-20{ }^{\circ} \mathrm{C}$. 


\subsection{3 $0.3 \%(w / v)$ DMACA-HCl solution}

To make 100 mL DMACA solution, dissolve 300 mg DMACA in $6 \mathrm{M} \mathrm{HCl:MeOH}$ (1:1). Mix and chilled till cold. Make DMACA solution fresh each time. 


\section{Appendix 2: Primer sequences}

\begin{tabular}{|c|c|c|}
\hline Primer name & Sequence $5^{\prime} \rightarrow 3^{\prime}$ (restriction site) & Organism \\
\hline ACT2 AS & AACGATTCCTGGACCTGCCTCATC & Arabidopsis thaliana \\
\hline ACT2 S & TCCCTCAGCACATTCCAGCAGAT & Arabidopsis thaliana \\
\hline $\mathrm{BvCYP}$ & $\begin{array}{c}\text { ccatcgatATGGATCATGCAACATTAG } \\
(\text { Cla })\end{array}$ & Beta vulgaris \\
\hline $\mathrm{BvCYP} 2$ & $\begin{array}{c}\text { cgggatccTCAATACCTAGGTATTGGA } \\
(\text { Bam } \mathrm{HI})\end{array}$ & Beta vulgaris \\
\hline DOD F & CATACTACCATGTCCACCAAG & Arabidopsis thaliana \\
\hline DOD R & AGCACTGCTTCTATATCGTC & Arabidopsis thaliana \\
\hline LBb1 & GCGTGGACCGCTTGCTGCAACT & Arabidopsis thaliana \\
\hline RTPCR ACT F & TCTATCCTTGCATCTCTCAG & Beta vulgaris \\
\hline RTPCR ACT R & ATCATACTCGCCCTTGGAGA & Beta vulgaris \\
\hline RTPCR CHS F & TGGTACCGTCCTTCGTTTAGCG & Beta vulgaris \\
\hline RTPCR CHS R & TCTCAGCACACACAACCAGCAC & Beta vulgaris \\
\hline RTPCR CYP F & CTTTTCAGTGGAATTAGCCCACC & Beta vulgaris \\
\hline RTPCR CYP R & CCCAATATCTTCCATAATGTTCCA & Beta vulgaris \\
\hline RTPCR DFR F & AGGCGCGAGCCTAAGGACTTCAA & Beta vulgaris \\
\hline RTPCR DFR R & TAGCCTGAAGCACCAGTCACGCA & Beta vulgaris \\
\hline RTPCR DOD1 F & CATTGGTTCAGGAAGTGCAA & Beta vulgaris \\
\hline RTPCR DOD1 R & ACGAAGCCATGAATCAAAGG & Beta vulgaris \\
\hline RTPCR DOD2 F & TGCATGGGTGCCTCTAATGCTG & Beta vulgaris \\
\hline RTPCR DOD2 R & TGCGATTGGACAGAGAGCTGAC & Beta vulgaris \\
\hline RTPCR EF2 F & AGTGGGCTTCGAAGGAAGGTGCT & Beta vulgaris \\
\hline RTPCR EF2 R & ACGGGCTCCAACAATCTGGGCT & Beta vulgaris \\
\hline RTPCR FLS F & TTTCTGTGTCCGGGCTTGGAAT & Beta vulgaris \\
\hline RTPCR FLS R & TGAAGCCCTCACCATCCTTGTT & Beta vulgaris \\
\hline RTPCR GT1 F & CGACAATAGAAGGGATCGCAGCAG & Beta vulgaris \\
\hline RTPCR GT1 R & AGGAATTGCTCGGCGAACACAG & Beta vulgaris \\
\hline RTPCR GT2 F & TTGGGCACCACAAGTCTCCATC & Beta vulgaris \\
\hline RTPCR GT2 R & ACCCACAATGCGATACGAACCC & Beta vulgaris \\
\hline SALK insert $F$ & CCGAGATAGGGTTGAGTGTTG & Arabidopsis thaliana \\
\hline SALK insert $\mathrm{R}$ & AAACTTGATTTGGGTGATGGTTC & Arabidopsis thaliana \\
\hline tt12 LP & TGCTTCCAAATGGATCATTTC & Arabidopsis thaliana \\
\hline $\mathrm{tt} 12 \mathrm{RP}$ & CAGTGTGGGAGTCAAAGCTTC & Arabidopsis thaliana \\
\hline
\end{tabular}




\title{
Appendix 3: DOD paper - co-author
}

\section{Betalain production is possible in anthocyanin- producing plant species given the presence of DOPA-dioxygenase and L-DOPA}

Nilangani N Harris ${ }^{1,4}$, John Javellana', Kevin M Davies', David H Lewis', Paula E Jameson², Simon C Deroles', Kate E Calcott ${ }^{1,3}$, Kevin S Gould ${ }^{3}$ and Kathy E Schwinn ${ }^{*}$

\begin{abstract}
Background: Carotenoids and anthocyanins are the predominant non-chlorophyll pigments in plants. However, certain families within the order Caryophyllales produce another class of pigments, the betalains, instead of anthocyanins. The occurrence of betalains and anthocyanins is mutually exclusive. Betalains are divided into two classes, the betaxanthins and betacyanins, which produce yellow to orange or violet colours, respectively. In this article we show betalain production in species that normally produce anthocyanins, through a combination of genetic modification and substrate feeding.

Results: The biolistic introduction of DNA constructs for transient overexpression of two different dihydroxyphenylalanine (DOPA) dioxygenases (DODs), and feeding of DOD substrate (L-DOPA), was sufficient to induce betalain production in cell cultures of Solanum tuberosum (potato) and petals of Antirhinum majus. HPLC analysis showed both betaxanthins and betacyanins were produced. Multi-cell foci with yellow, orange and/or red colours occurred, with either a fungal DOD (from Amanita muscaria) or a plant DOD (from Portulaca grandiffora), and the yellow/orange foci showed green autofluorescence characteristic of betaxanthins. Stably transformed Arabidopsis thaliana (arabidopsis) lines containing 35S: AmDOD produced yellow colouration in flowers and orangered colouration in seedlings when fed L-DOPA. These tissues also showed green autofluorescence. HPLC analysis of the transgenic seedlings fed L-DOPA confirmed betaxanthin production.

Conclusions: The fact that the introduction of DOD along with a supply of its substrate (L-DOPA) was sufficient to induce betacyanin production reveals the presence of a background enzyme, possibly a tyrosinase, that can convert L-DOPA to cyclo-DOPA (or dopaxanthin to betacyanin) in at least some anthocyanin-producing plants. The plants also demonstrate that betalains can accumulate in anthocyanin-producing species. Thus, introduction of a DOD and an enzyme capable of converting tyrosine to L-DOPA should be sufficient to confer both betaxanthin and betacyanin production to anthocyanin-producing species. The requirement for few novel biosynthetic steps may have assisted in the evolution of the betalain biosynthetic pathway in the Caryophyllales, and facilitated multiple origins of the pathway in this order and in fungi. The stably transformed 35S: AmDOD arabidopsis plants provide material to study, for the first time, the physiological effects of having both betalains and anthocyanins in the same plant tissues.
\end{abstract}

\footnotetext{
* Correspondence: Kathy.Schwinn@plantandfood.co.nz

'New Zealand Institute for Plant \& Food Research Limited, Private Bag 11-

600 , Palmerston North, New Zealand

Full list of author information is available at the end of the article
}

• 2012 Harris et al; licensee BioMed Central Ltd. This is an Open Access article distributed under the terms of the Creative Commons Attribution License (http://creativecommons.org/licenses/by/2.0), which permits unrestricted use, distribution, and reproduction in any medium, provided the original work is properly cited. 


\section{Background}

The variety of colours observed in flowers, fruits and vegetative tissues in plants are due to the presence of chromogenic plant secondary metabolites $[1,2]$. These pigments serve diverse functions including photosynthesis and the protection of the photosynthetic machinery, attraction of pollinators and seed dispersers, and protection against biotic and abiotic stresses $[1,3]$. In addition to their biological functions, plant pigments are also of much interest regarding their possible beneficial effects on human health, their use as natural colorants and their aesthetic value in ornamental and food crops [4]. Non-chlorophyll plant pigments predominantly belong to two groups: flavonoids and carotenoids. Within the flavonoids, anthocyanins are the most significant type, providing a range of colours including orange, red, pink, mauve, purple and blue. However, in certain families within the order Caryophyllales, another class of pigments, the betalains, replaces the anthocyanins $[2,5,6]$. Betalains are only present in the order Caryophyllales and some fungi. They occur in most families of the Caryophyllales, but species of at least two families accumulate anthocyanin pigments instead [7]. The basis of this differentiation is unknown, but may represent an initial evolution of betalain biosynthesis in an ancestor of the core Caryophyllales and then its subsequent loss on different occasions [7].

No plant has yet been found that produces both betalain and anthocyanin pigments [5-8]. This mutually exclusive nature of the betalain and anthocyanin production in the plant kingdom is a curious phenomenon and the evolutionary and biochemical mechanisms for this restriction are unknown [5-7].

There are two major types of betalains, the red-purple betacyanins and the yellow/orange betaxanthins, both of which accumulate in the vacuole. The betaxanthins also emit green autofluorescence, which is not seen with the betacyanins [9-11]. While the production of flavonoids and carotenoids has been extensively studied and metabolically engineered in a variety of species, betalain biosynthesis has yet to be fully characterised $[1,2]$. The betalain biosynthetic pathway is relatively simple with putatively only a few reactions that are enzyme catalysed (Figure 1). The initial biosynthetic step is the hydroxylation of tyrosine to L-3,4-dihydroxyphenylalanine (DOPA), attributed to the activity of a tyrosinase, although the exact role (if any) of tyrosinase in betalain synthesis has yet to be resolved $[5,6,12,13]$ Cleavage of the cyclic ring of L-DOPA by DOPA-4,5-dioxygenase (DOD) forms an unstable seco-DOPA intermediate, which is thought to spontaneously convert to betalamic acid. The formation of betaxanthins occurs spontaneously from the condensation of betalamic acid with

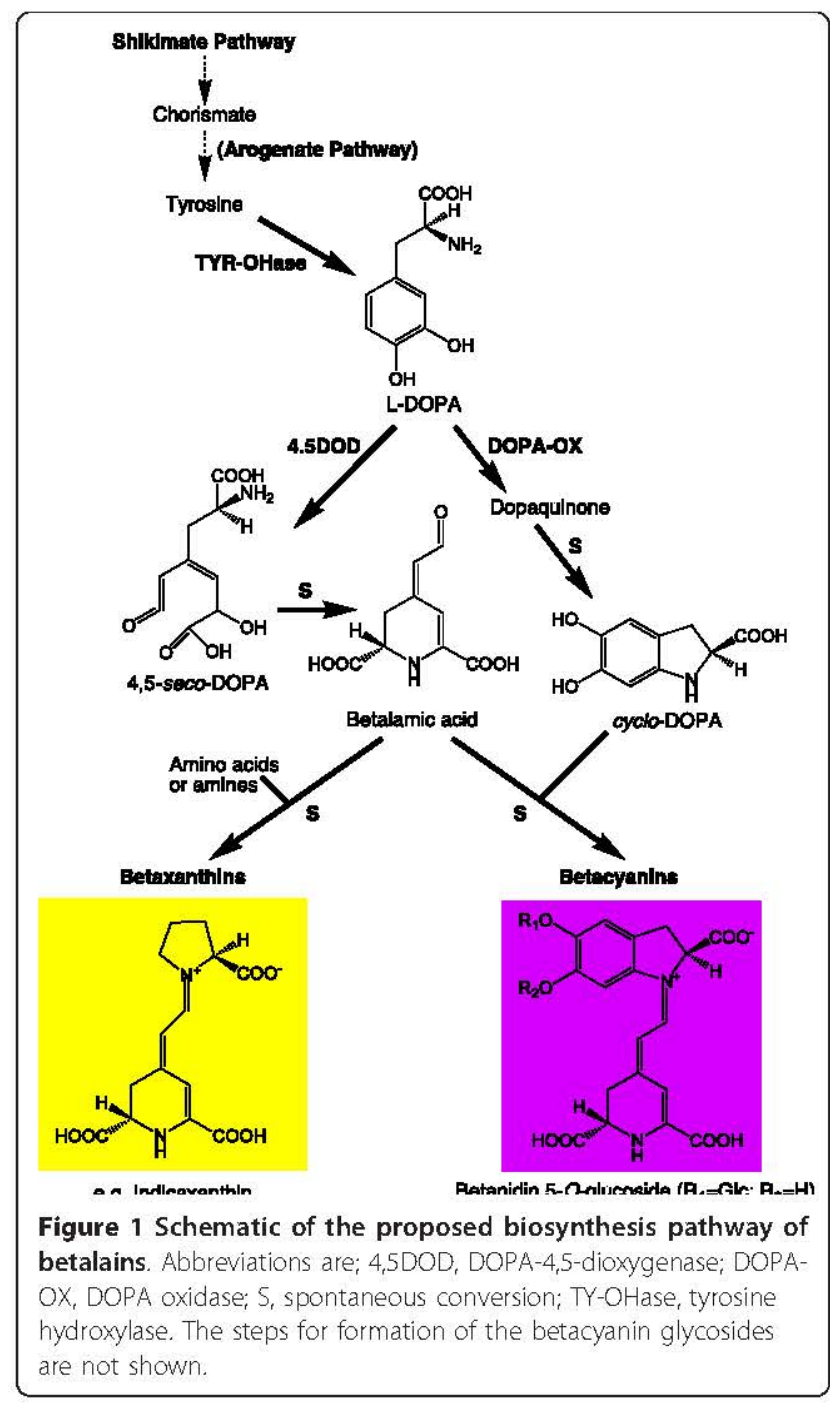

amines/amino acids [14], probably in the vacuole. The classic model of betacyanin biosynthesis involves the condensation of betalamic acid with (most commonly) cyclo-DOPA, which again is a likely spontaneous step. In this model, cyclo-DOPA is formed from L-DOPA through an oxidation reaction that also has been attributed to tyrosinase activity $[5,6,13]$ The conversion proceeds via an unstable dopaquinone intermediate, which spontaneously cyclizes to form cyclo-DOPA. Betacyanins are generally $\mathrm{O}$-glycosylated (at the $\mathrm{C}-5$ or $\mathrm{C}-6$ ) and frequently subsequently acylated. The aglycone product of betalamic acid and cyclo-DOPA condensation is termed betanidin and the 5-O-glucosylated form betanin, as with the anthocyanidin/anthocyanin convention. The timing of the glycosylation, regarding whether it occurs on cyclo-DOPA or betanidin, has been debated $[15,16]$.

The only betalain biosynthetic enzymes characterised at the molecular level to date are for DOD and some of 
the glycosyltransferase activities. The first plant $D O D$ gene characterised was from Portulaca grandiflora, and it defined a novel plant gene family of non-haem dioxygenases $[2,11]$. The plant dioxygenase is phylogenetically unrelated to DOD from the fungi Amanita muscaria, although the fungal sequence can complement betalain production in flowers of a $P$. grandiflora dod mutant [17]. The plant DOD carries out only the 4,5-cleavage of DOPA to yield betalamic acid, whereas the fungal enzyme can also conduct 2,3-extradiol cleavage of DOPA, yielding the yellow pigment muscaflavin [18]. Three cDNAs encoding proteins with relevant $O$-glycosylation activity have been identified, two that use both betanidin and flavonoids as substrates $[19,20]$ and one that uses cyclo-DOPA [16].

Studies seeking to understand the genetic basis of the mutual exclusion of betalains and anthocyanins have focused on establishing the extent of the retention of the anthocyanin biosynthetic pathway in betalain producing species [21-23]. Flavonoids are present in betalain producing species, including flavonols and proanthocyanidins, and functional genes have been identified for the flavonoid biosynthetic enzymes chalcone synthase, dihydroflavonol 4-reductase and anthocyanidin synthase [21-23]. This suggests that the lack of anthocyanin production in betalain-producing species may be due to a lack of transcriptional activation of all the necessary biosynthetic genes $[7,23]$, although a hypothesis based on repressive interaction between anthocyanin and betalain metabolites and the biosynthetic enzymes has also been suggested [24].
Our aim in this study was to determine whether betalain production is possible in anthocyanin-producing species. Using genetic transformation and feeding of pathway intermediates, we have examined what the minimum number is of biosynthetic steps that must be introduced into an anthocyanin-producing species to allow betalain production, and whether betalains can accumulate to significant levels in such species. Stable or transient transgene expression was used with DOD cDNAs from the fungus $A$. muscaria and the plant P. grandiflora, introduced into Arabidopsis thaliana (arabidopsis) plants, Solanum tuberosum (potato) cell cultures and Antirrhinum majus (antirrhinum) petals. These are representatives of the asterids and rosids, the two major clades of eudicots.

\section{Results and discussion}

Betalain biosynthesis in potato cell cultures by transient expression of $D O D$

Potato cell suspension cultures were transformed using particle bombardment with 35S: green fluorescent protein $(G F P)$ or constructs having either the $P$. grandiflora DOD cDNA (35S: PgDOD) or the A. muscaria DOD (35S: $A m D O D)$ driven by the CaMV35S promoter, and examined for betalain production following feeding with L-DOPA. In cells transformed with 35S: GFP, GFP was detected in the cells $24 \mathrm{~h}$ after biolistic transformation but no pigmentation was apparent (data not shown). In the cells transformed with either 35S: PgDOD or 35S: $A m D O D$ and fed L-DOPA, pigmented multi-celled clusters were apparent within $24 \mathrm{~h}$ post-bombardment (Figure 2). Both 35S: AmDOD and 35S: PgDOD resulted

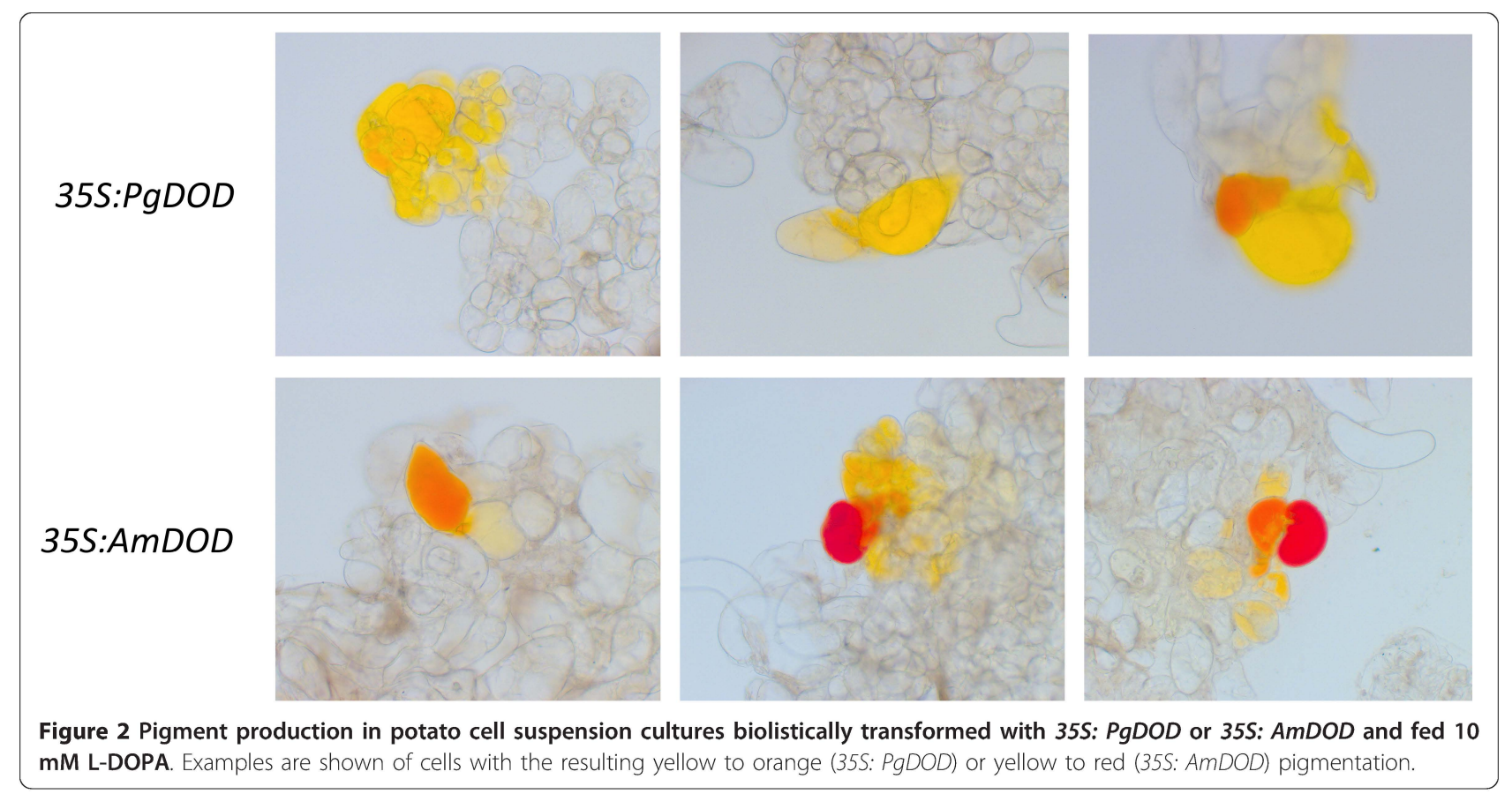


in yellow and orange cell clusters. In addition, 35S: $A m D O D$ also produced cells with red pigmentation. The yellow and red pigmentation suggests that betaxanthin and betacyanin production, respectively, had been conferred to the cells. Indeed, observation of the yellow pigmented areas under blue light showed the autofluorescence characteristic of betaxanthin pigments (data not shown).

\section{Betalain biosynthesis in antirrhinum petals by transient expression of $D O D$}

The adaxial surface of dorsal petals of antirrhinum was transformed using particle bombardment with $35 \mathrm{~S}$ : $P g D O D$ and examined for betalain production with or without infiltration of the petals with L-DOPA. Petals biolistically transformed with 35S: GFP vector and fed with L-DOPA were used as an additional control. Antirrhinum lines having mutations in flavonoid production were used to provide anthocyanin-free petal backgrounds upon which to observe any pigment production. No pigments were visible in 35S: GFP shot tissue or in petals transformed with 355 : PgDOD but not infiltrated with L-DOPA, although positive GFP foci were apparent (Figure 3). The 35S: PgDOD petals that were infiltrated with L-DOPA had numerous, multi-celled yellow foci $24 \mathrm{~h}$ after infiltration ( $48 \mathrm{~h}$ after bombardment) (Figure 3), indicating betaxanthin production. The yellow foci did indeed have the strong green autofluorescence typical of betaxanthins when observed under blue light (Figure 3). When using the 35S: $A m D O D$ construct, both yellow/orange and pink/red multi-celled foci occurred (Figure 4), although the pink/ red foci were present only inconsistently (data not shown). Similarly, pink foci were sometimes present in some replicate experiments using 35S: PgDOD (data not shown). The pink colouration indicates betacyanin production. The pink regions did not show strong autofluorescence while the central cells did, indicating possible betacyanin accumulation around a central region containing both betacyanins and betaxanthins.

HPLC analysis (LC-DAD) was used to examine the nature of the yellow pigments produced following bombardment with 35S: PgDOD. The ridge region of the petals was chosen for HPLC analysis, to ensure a similar region was sampled in each case. Betaxanthins and betacyanins have absorbance maxima at around $470 \mathrm{~nm}$ and $538 \mathrm{~nm}$, respectively [25], and peak profiles were examined at these two wavelengths (Figure 5). No betalain-

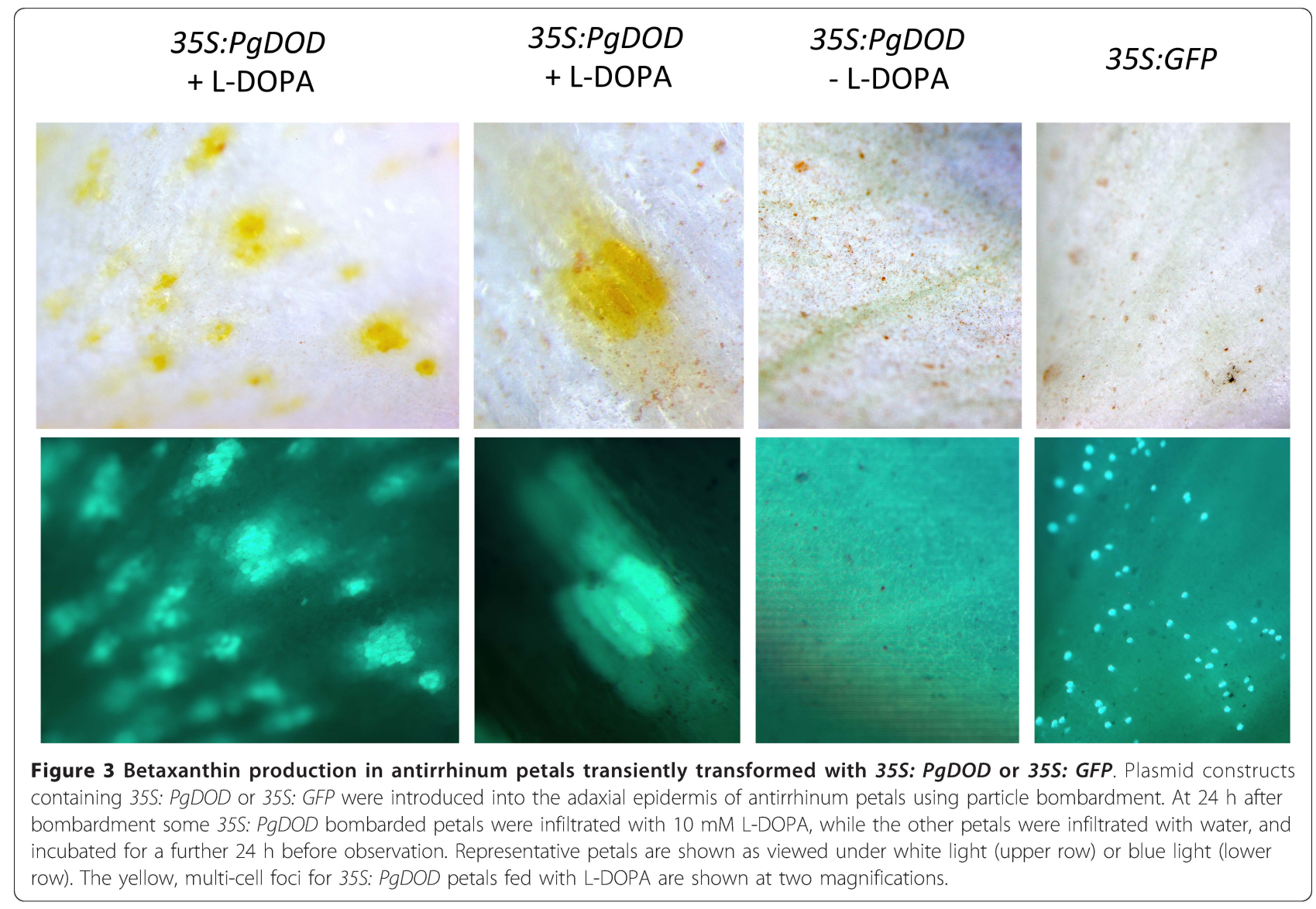




\section{S:AmDOD}

\section{White light}

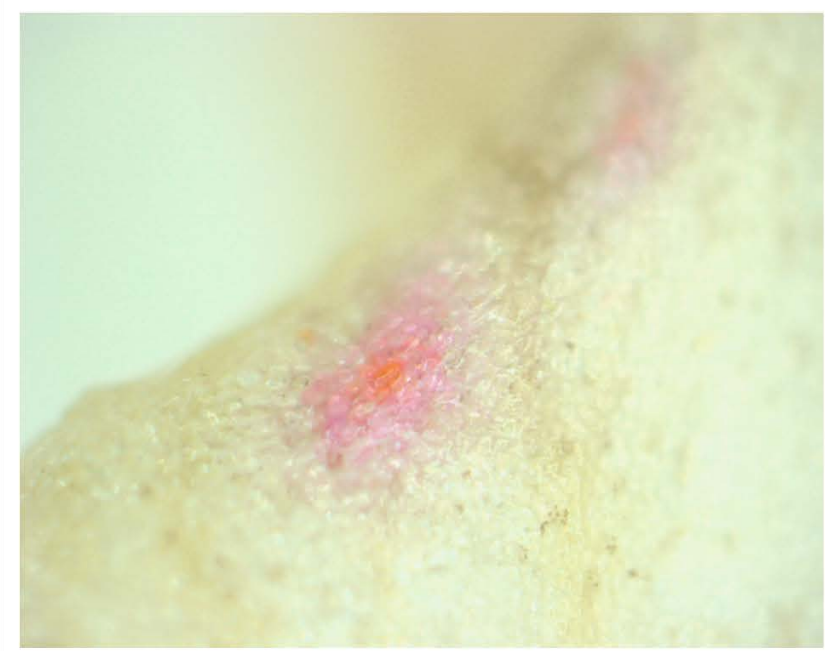

Blue light

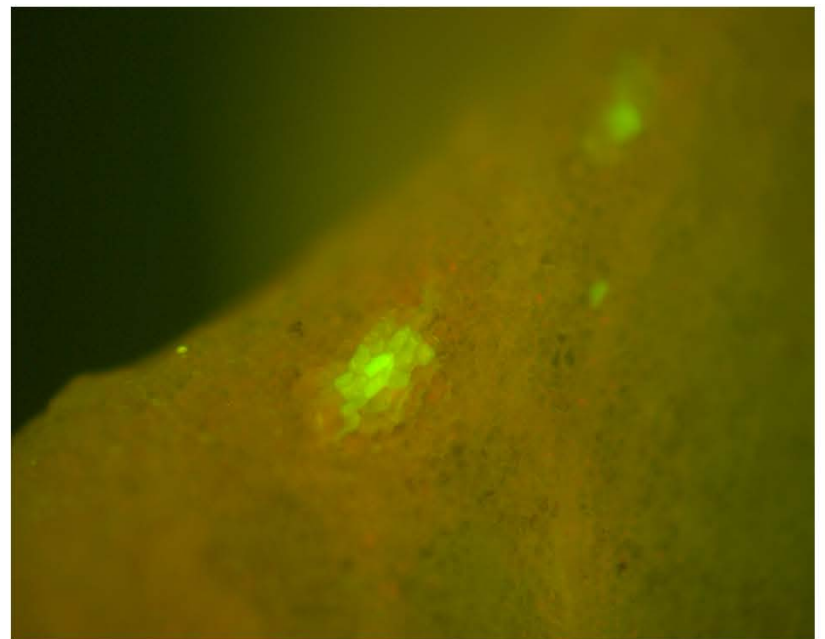

Figure 4 Pigment production in antirrhinum petals transiently transformed with 355 : AmDOD. A plasmid construct containing 35S: AmDOD was introduced into the adaxial epidermis of antirrhinum petals using particle bombardment. At 24 h after bombardment the bombarded petals were infiltrated with $10 \mathrm{mM} \mathrm{L-DOPA}$ and incubated for a further $24 \mathrm{~h}$ before observation. A representative petal is shown as viewed under white light (left) or blue light (right).

related peaks were detected with HPLC analysis of the 35S: GFP petal tissue or with 35S: $P g D O D$ petals that were not infiltrated with L-DOPA. Petals transformed with 35S: $P g D O D$ and fed L-DOPA showed distinct peaks at both $470 \mathrm{~nm}$ and $538 \mathrm{~nm}$. The compounds represented by the peaks were putatively identified by comparison of HPLC retention times and spectral data against those of a standard extract (from beetroot, Figure 5A and 5E) and reported spectral data [14,25,26]. The beetroot extract showed the expected peaks for the betaxanthin vulgaxanthin I (Peak 1 ) and the betacyanins betanin (betanidin 5-O-glucoside, Peak 2) and isobetanin (Peak 3) (Table 1). The 35S: PgDOD L-DOPA fed samples showed a small amount of betanin (Peak 2; Figure 5F) and an unknown peak (Peak 5, $21.02 \mathrm{~min}$; Figure $5 \mathrm{~B})$ that was possibly dopaxanthin (Table 1). Generally, the chromatograms at $538 \mathrm{~nm}$ showed the same patterns as at $470 \mathrm{~nm}$ except that vulgaxanthin I (Peak 1) and the putative dopaxanthin peak (Peak 5) were no longer detected, providing further evidence that Peak 5 is indeed a betaxanthin.

Antirrhinum can produce yellow pigments in the petal face and throat naturally. These pigments are aurones and a product of the flavonoid pathway. In addition to the spectral data (aurones have spectra maxima in the range $390-430 \mathrm{~nm}$ ), there is other evidence that the yellow pigments observed following 35S: $P g D O D$ bombardment and L-DOPA feeding are unlikely to be aurones; aurones are not present normally in the dorsal petals of the antirrhinum line used (J119), and no yellow pigments were observed without infiltration with L-DOPA.

Despite a lack of the red colouration seen following bomardment, the $35 S$ : $P g D O D$ antirrhinum samples analysed by HPLC did indeed contain small amounts of betacyanin (betanin; Figure 5F). Also, the presence of betacyanin is inferred in the 35S: $A m D O D$ expressing potato cells, given the red colouration of some foci. How betacyanin production can occur in non-betalain species through the introduced DOD acting on the supplied L-DOPA is not clear. The product of DOD action on L-DOPA is betalamic acid (Figure 1). Betaxanthins can be produced by spontaneous reactions of the DOD reaction end-product, betalamic acid, with amino acids or amines. However, betacyanin formation requires cycloDOPA, the formation of which from L-DOPA by oxidation has been attributed to the activity of a tyrosinase [6]. As tyrosinases are frequently present in plant cells it is possible that an endogenous enzyme with activity on $\mathrm{L}$ DOPA occurs in most non-betalain producing species. Alternatively, betacyanin could be formed from endogenous tyrosinase activity on dopaxanthin [12]. The betacyanin detected in antirrhinum petals was likely betanin, an $O$-glycosylated betacyanin. This indicates that endogenous glycosyltransferases can act on the novel betacyanin substrates. Two O-glucosyltransferases have been characterized with activity on betanidin $[19,20]$. Both show sequence similarity to the $O$-glycosyltransferases involved in anthocyanin biosynthesis, and indeed, the betanidin 

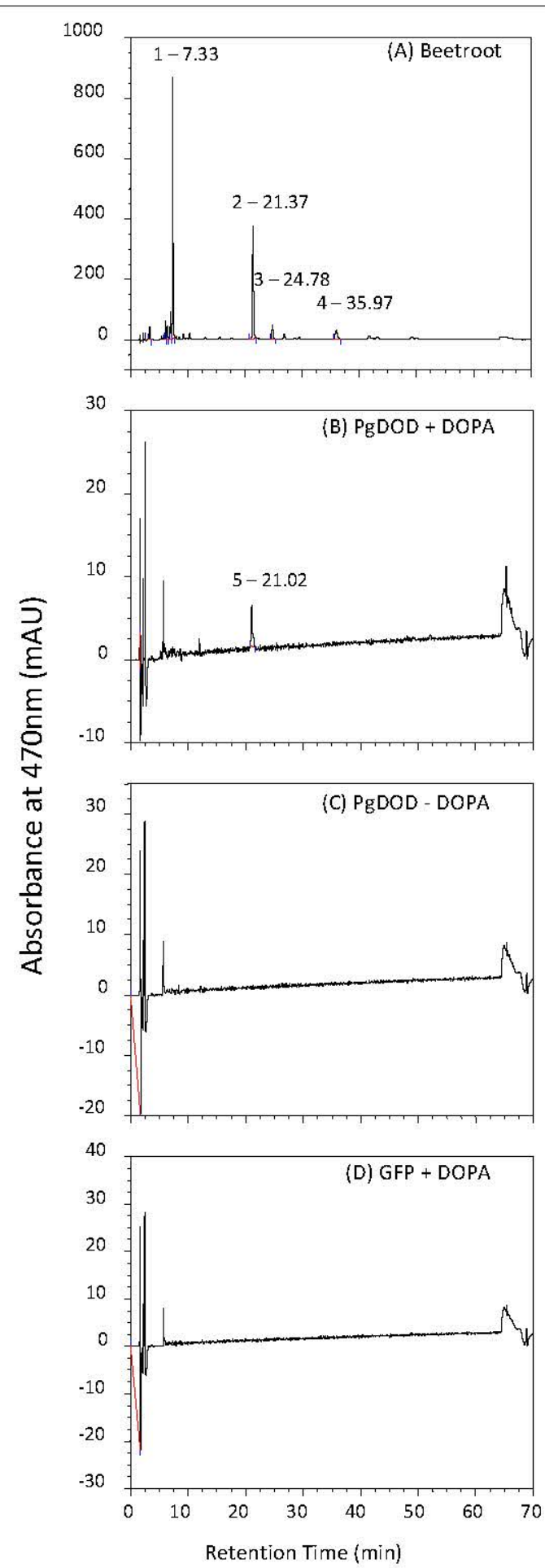
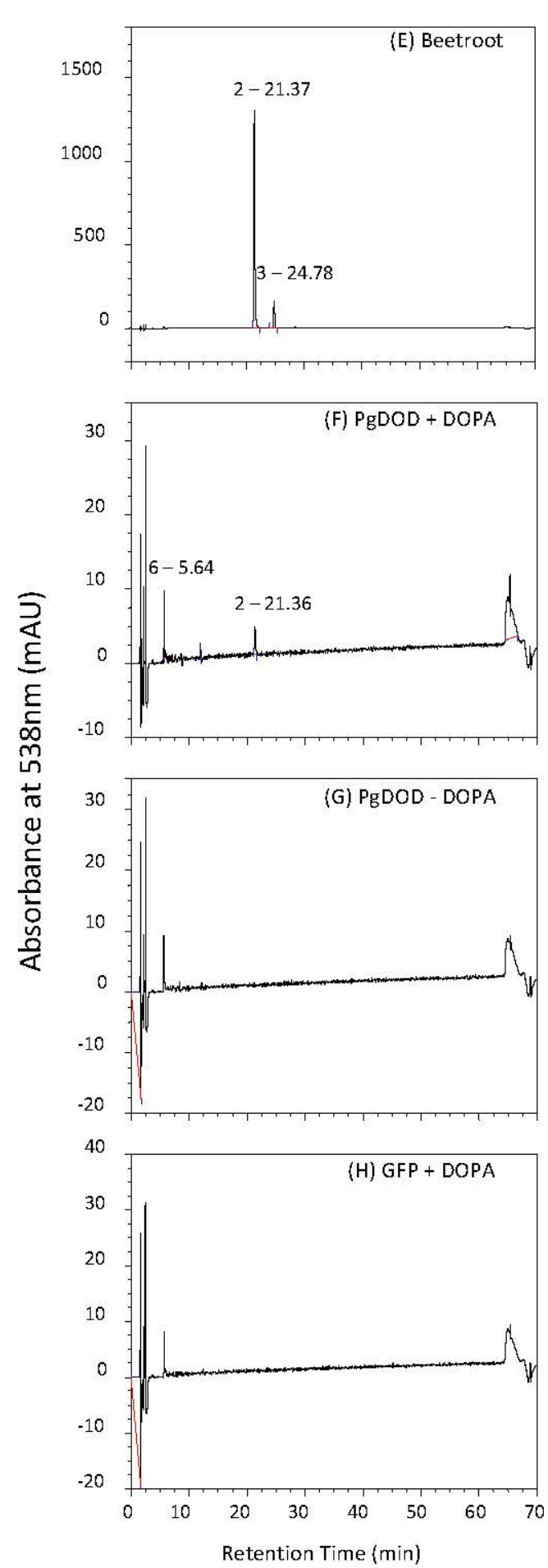

Figure $5 \mathrm{HPLC}$ analysis of pigments produced in antirrhinum petals transiently transformed with 35S: PgDOD or 35S: GFP. HPLC analysis was conducted on the 355: PgDOD and 355: GFP antirrhinum petal material shown in Figure 3. Absorbance was monitored at $470 \mathrm{~nm}$ for betaxanthins or $538 \mathrm{~nm}$ for betacyanins. Chromatograms are for extracts from beetroot root ( $\mathrm{A}$ and E), 355: PgDOD antirrhinum infiltrated with L-DOPA (B and F), 355: PQDOD antirrhinum without L-DOPA infiltration ( $C$ and G), 355: GFP antirrhinum infiltrated with L-DOPA (D and H). The retention times of the major peaks are shown, and these were tentatively identified as per Table 1. 
Table 1 Retention times and spectral maxima of the major betalain pigments detected in beetroot tissue (Peaks 1 to 3) or antirrhinum petal tissue bombarded with 35S: PgDOD and infiltrated with L-DOPA (Peaks 4 and 5).

\begin{tabular}{llll}
\hline Peak $^{\text {a }}$ & Betalain & Retention Time $(\mathbf{m i n})$ & $\boldsymbol{\lambda}_{\max }(\mathbf{n m})$ \\
\hline 1 & Vulgaxanthin I & 7.33 & 259,469 \\
\hline 2 & Betanin & 21.37 & 269,534 \\
\hline 3 & Isobetanin & 24.78 & 269,534 \\
\hline 4 & Unknown betaxanthin $^{\prime}$ & 35.97 & 265,464 \\
\hline 5 & Unknown betaxanthin $^{\text {b }}$ & 21.02 & 241,470 \\
\hline
\end{tabular}

HPLC absorbance traces are shown in Figure 4

The peak present at $5.64 \mathrm{~min}$ in antirrhinum tissue was present in all samples, both control and transgenic; its spectral data is not consistent with that of betalain pigments therefore it was excluded from the analysis.

${ }^{b}$ Possibly dopaxanthin

5-O-glucosyltransferase has activity with both betacyanins and flavonoids [20]. Thus, it may be the case that the endogenous flavonoid $O$-glucosyltransferases of antirrhinum are also able to act on betanidin (and/or cycloDOPA).

Biolistic transformation of both potato and antirrhinum with either $35 S: P g D O D$ or $35 S: A m D O D$ resulted in muli-celled foci producing betalains. It is unlikely that this would be due to movement of the DOD enzyme between cells as, similar to GFP (Figure 3), it is too large for passive intercellular movement. As the final betalain pigments accumulate within the vacuole, this suggests that some of the precursors migrate between cells. This is similar to the results of Mueller et al. [17] when they biolistically introduced $A m D O D$ into different $P$. grandiflora mutant backgrounds. Single coloured cells seen within $18 \mathrm{~h}$ after bombardment developed into multi-cell foci by $48 \mathrm{~h}$ after bombardment [17]. It was suggested that betalains could have diffused through plasmodesmata to neighbouring cells. However, in contrast to these results, when complementation of the $P$. grandiflora dod mutant was conducted with PgDOD, only single cell foci occurred [11]. Furthermore, cell-specific betalain production is commonly observed in plants, such as in the epidermal cells of petals. In the case of our results, it is possible that high L-DOPA levels produced from tissue feeding allowed the movement of betalamic acid not just to the vacuole but also to neighbouring cells.

Betalain pigment production by stable transformation of Arabidopsis

Stably transformed arabidopsis plants were produced through Agrobacterium-mediated transformation with 35S: $A m D O D$. T2 generation seedlings were checked for expression of the $35 S$ : $A m D O D$ transgene (Additional file 1 ) and selected lines examined for their potential to produce betalains when fed with L-DOPA. When whole seedlings were fed L-DOPA, novel pigment production was visible within $12 \mathrm{~h}$ after feeding (Figure 6A-C), including in the etiolated root tissues and the hypocotyls. The colour ranged from pale yellow through to orange and dark orange-red. Under blue light, the pigments showed the green autofluorescence characteristic of betaxanthins (Figure 6D, E). When detached inflorescences from mature plants were fed L-DOPA pale yellow pigmentation was seen in all tissues $24 \mathrm{~h}$ after feeding, including the stem, petals and siliques, and this was accompanied by strong autofluorescence (Figure 7). 35S: $A m D O D$ seedlings or inflorescences not fed $\mathrm{L}$ DOPA did not produce visible pigments after treatment and did not show significant autofluorescence under blue light (Figure 6F, G and Figure 7).

HPLC analysis was used to examine the nature of the pigments produced following L-DOPA feeding of the 35S: $A m D O D$ arabidopsis. Seedlings were fed L-DOPA and the entire seedling sampled for chemical analysis. HPLC analysis revealed several peaks present in the $\mathrm{L}$ DOPA fed 35S: AmDOD tissue but not in the control tissue (Figure 8). Four of these peaks were present in sufficient quantity to confirm that their spectral data and retention times were those characteristic of betaxanthins (Table 2).

The presence of betaxanthins matched the strong autofluorescence observed in the seedlings under blue light. The failure to detect the presence of betacyanin was a surprise given the orange/red colouration of the seedlings and the ability of $D O D$ to confer both betaxanthin and betacyanin production when transiently expressed in antirrhinum petals. In accordance with the HPLC results, while the seedlings did have an orange-red colour that would suggest betacyanin production, the autofluorescence indicated strong betaxanthin presence. Furthermore, the fed inflorescences off the mature plants did not have a red colouration. Thus it appears that a high level of betaxanthin production in the particular background colouration of the seedlings resulted in the orange-red colour. It is unlikely that anthocyanins are contributing to the orange-red colouration of seedlings, as L-DOPA treatment of non-transgenic seedlings did not induce visible pigment production.

\section{Conclusions}

We report here methods to enable production of highlevels of betalains in the non-betalain, anthocyanin-producing, species antirrhinum, arabidopsis and potato, which represent both of the major clades of the eudicots. Betalains are not normally observed in anthocyanin-producing species, for unknown reasons.

The introduction of a single enzyme (DOD) and feeding of that enzyme's substrate (L-DOPA) were 


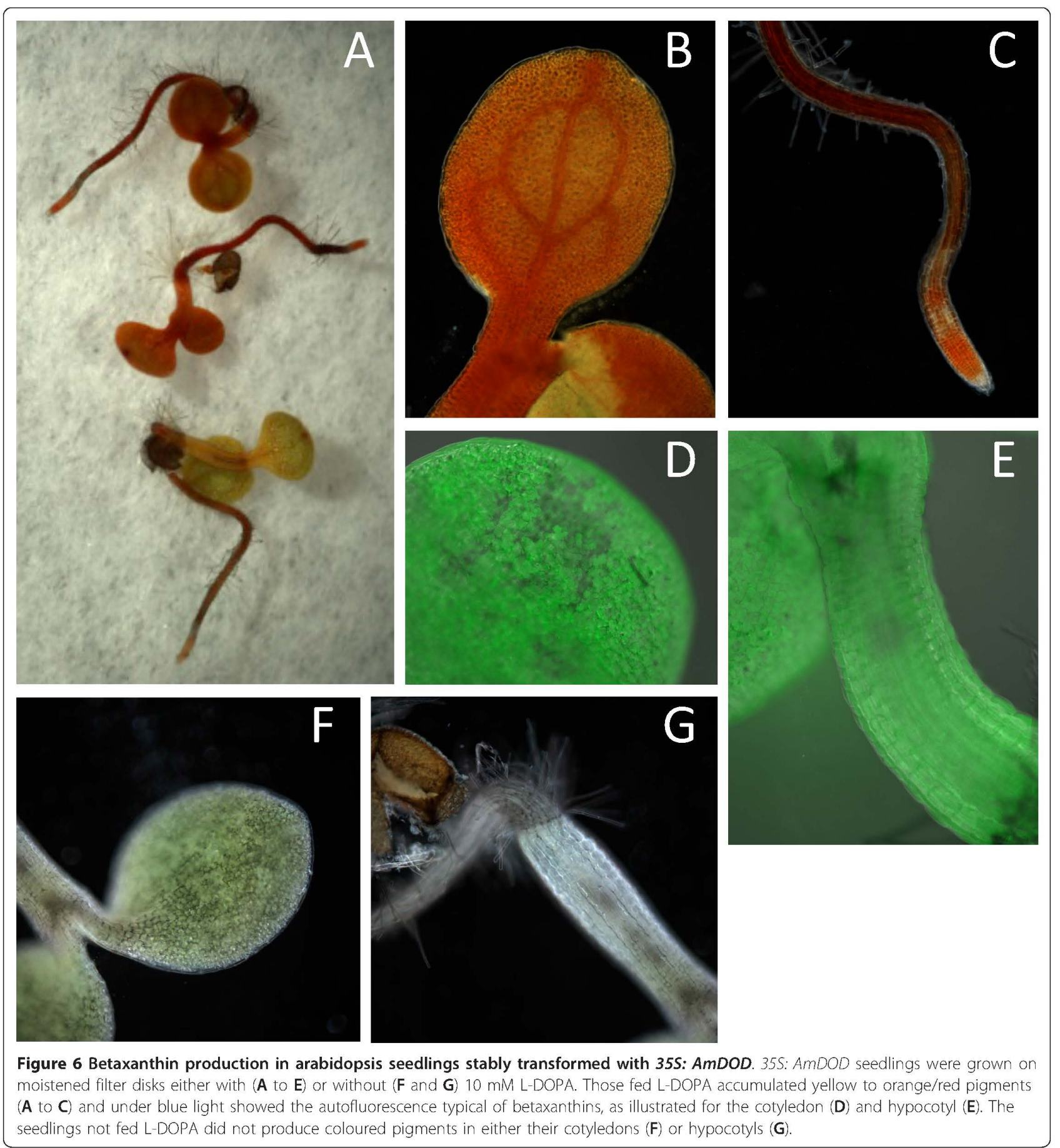

sufficient to induce both betaxanthin and betacyanin production. Although many steps in betalain production are suggested to be spontaneous, it is somewhat surprising that betacyanin production occurred. It indicates the background presence of an enzyme able to convert L-DOPA to cyclo-DOPA, or dopaxanthin to betacyanin/betanidin, and $O$-glycosyltransferases with activity against betanidin. Thus, it is likely that introduction of just two enzymes, DOD and the (currently uncharacterised) enzyme for conversion of tyrosine to L-DOPA, should be sufficient to confer betaxanthin and betacyanin production to anthocyanin-producing species. The requirement for few novel biosynthetic steps may have assisted in the initial evolution of this pathway in the Caryophyllales. A relatively simple mechanism for betalain biosynthesis 

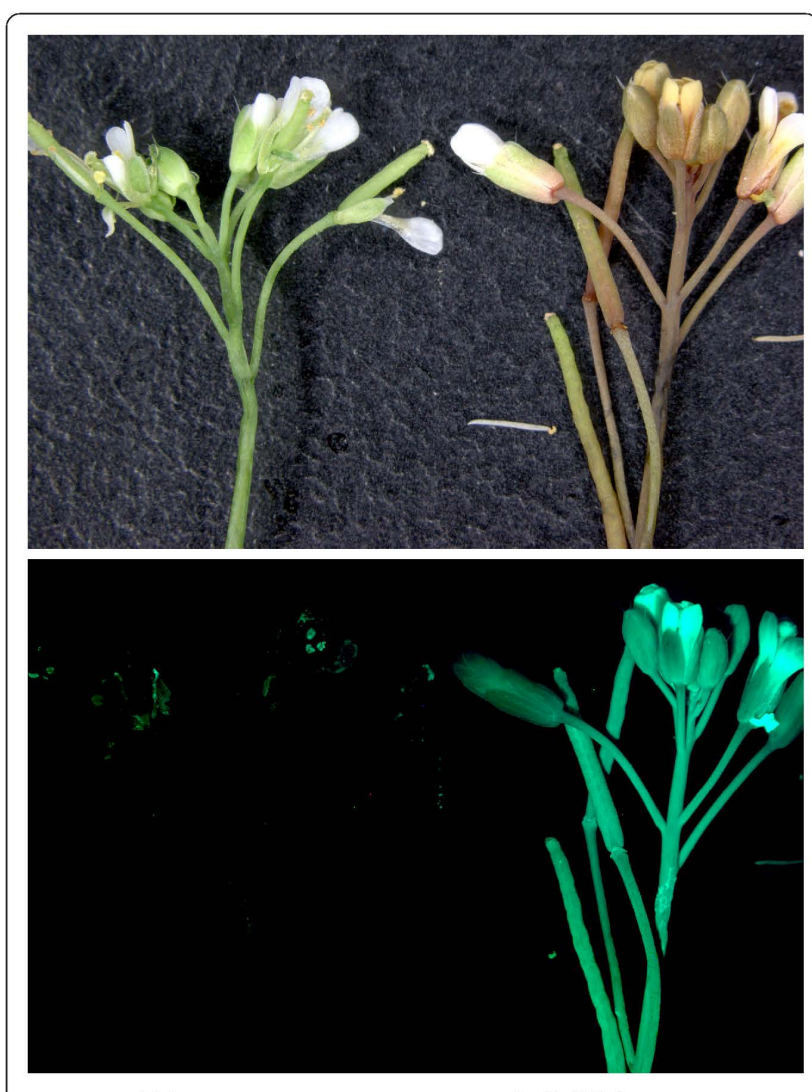

Water

L-DOPA

Figure 7 Pigment production in inflorescences of arabidopsis plants stably transformed with 355 : AmDOD. Inflorescences from 35S: AmDOD arabidopsis line 6 were infiltrated with either water or $10 \mathrm{mM}$ L-DOPA for $24 \mathrm{~h}$ and examined under white light (upper panel) or blue light (lower panel). Those fed L-DOPA accumulated yellow to orange pigments and under blue light showed the autofluorescence typical of betaxanthins. The inflorescences not fed L-DOPA did not produce visible coloured pigments and did not show autofluorescence.

would also support the multiple origins of the pathway in the Caryophyllales and in some fungi [7].

Given the extensive knowledge available on anthocyanin production and function in arabidopsis, the stably transformed 35S: AmDOD arabidopsis plants should provide excellent material to study, for the first time, the physiological effects of having both betalains and anthocyanins in the same plant tissues. The large collection of mutant lines in arabidopsis should also allow aspects of betalain biosynthesis, such as vacuolar transport, to be examined.

\section{Methods}

Plant and cell culture material

Antirrhinum majus plants were grown under standard glasshouse conditions in Palmerston North, New Zealand. The glasshouse was heated at $15^{\circ} \mathrm{C}$ and vented at

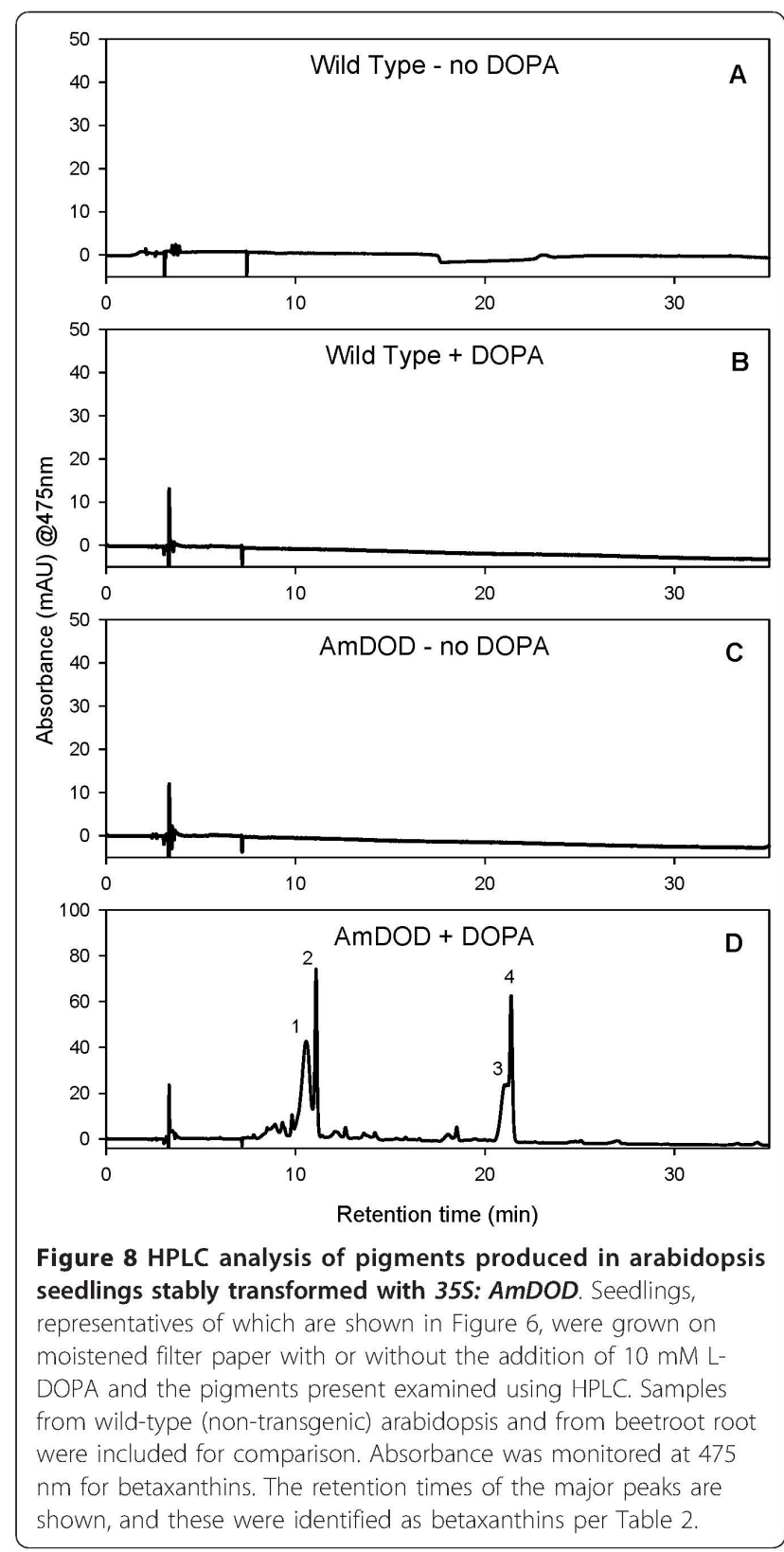

Table 2 Retention times and spectral maxima of the major peaks detected by HPLC using samples from 35S: AmDOD plants fed $10 \mathrm{mM}$ L-DOPA

\begin{tabular}{llll}
\hline Peak & Betalain $^{\text {a }}$ & Retention Time $(\mathbf{m i n})$ & $\boldsymbol{\lambda}_{\max }(\mathbf{n m})$ \\
\hline 1 & Vulgaxanthin $^{b}$ & 10.4 & 268,468 \\
\hline 2 & Unknown betaxanthin & 11.6 & 262,470 \\
\hline 3 & Unknown betaxanthin & 20.4 & 264,472 \\
\hline 4 & Unknown betaxanthin & 21.7 & 262,468 \\
\hline
\end{tabular}

${ }^{a}$ Spectral data for all four peaks was generally consistent with data reported previously for betaxanthins, but no specific betaxanthin assignments were made.

${ }^{\mathrm{b}}$ Peak one has a retention time and spectrum consistent with that of

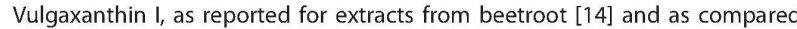
against a beetroot extract run in the same experimental set. 
$25^{\circ} \mathrm{C}$, without supplementary lighting. Two antirrhinum lines were used. Transformation with 35S: PgDOD used line II19, which produces aurones and flavones but not anthocyanins in the petals, as it is lacking in flavanone 3-hydroxylase activity (homozygous incolorataII). 35S: AmDOD transformation used either JI19 or rosea ${ }^{\text {dorsea, }}$, which carries a mutation in the R2R3MYB gene Rosea1 and lacks anthocyanin production in the adaxial epidermis of the petals [27]. Arabidopsis thaliana transformation used the Colombia ecotype. Potato cell cultures were obtained by placing $2 \mathrm{~cm}^{2}$ pieces of potato callus in $50 \mathrm{~mL}$ of modified liquid MS media [28] per cottonplugged $250 \mathrm{~mL}$ flask. These cultures were incubated at room temperature $\left(23-27^{\circ} \mathrm{C}\right)$ with shaking on an orbital shaker at $100 \mathrm{rpm}$ in the dark. Subculturing was carried out every two weeks with $3 \mathrm{~mL}$ of the cell culture used to inoculate a fresh $50 \mathrm{~mL}$ of liquid media.

\section{PCR cloning of P. Grandiflora DOD CDNA and vector construction}

A CDNA for the ORF of DOD was RT-PCR amplified from mRNA prepared from $P$. grandiflora betalain pigmented petals using primers designed to the published sequence [11]. The forward primer was 5-AGTCAGAATCCATGGGTGTTGGGAAGGAA-3', with the first ATG matching the initiation ATG for DOD, and the reverse primer was $5^{\circ}$-AGTCATCTAGAATCATATGGAAGTGAACT-3', which incorporated an XbaI site. Standard PCR conditions were used with Taq Polymerase (New England Biolabs, Massachusetts, USA) and cycling parameters of $94^{\circ} \mathrm{C}$ for $4 \mathrm{~min}$, then 30 cycles of $94^{\circ} \mathrm{C}$ for $30 \mathrm{~s}, 60^{\circ} \mathrm{C}$ for $30 \mathrm{~s}$ and $72^{\circ} \mathrm{C}$ for $1 \mathrm{~min}$, with a final extension phase of $72^{\circ} \mathrm{C}$ for $7 \mathrm{~min}$. The products were cloned into pGEM-T-Easy (Promega, Wisconsin, USA) and confirmed as $P g D O D$ by DNA sequencing. The cDNA was then excised as an EcoRI/XbaI fragment and ligated into EcoRI/XbaI digested $p A R T 7$ [29] to from the vector pPN314. The 35S: $A m D O D$ construct for particle bombardment, containing the $A$. muscaria 4,5-DOPA dioxygenase cDNA driven by the CaMV35S promoter, was $p N c o D o d$ [17] (courtesy of Dr. Willibald Schliemann, Leibniz-Institute of Plant Biochemistry, Germany). The binary vector $p P N 166$ for transformation into arabidopsis was constructed by taking the CaMV35S-cDNA region from $p N c o D o d$ as a PvulI fragment and cloning it into the NotI site (after flushing by end-filling) in the binary vector $p M L B A R T$ (a gift from Dr Bart Janssen, Plant \& Food Research). The 35S: GFP construct was pPN93. Vectors were verified by restriction fragment analysis and/or DNA sequencing.

\section{Biolistic transformation}

Antirrhinum particle bombardment experiments were performed as described in Shang et al. [30], with the following variations; the pressure setting was 300 or 400 $\mathrm{kPa}$, the shooting distance $11-12 \mathrm{~cm}$, and petals were bombarded twice. The final DNA concentration for DOD constructs was 1 or $2 \mu \mathrm{g}$ DNA per mg of $1.0 \mu \mathrm{m}$ gold particles. Controls included gold particles alone (no DNA) and 35S: GFP (added at $0.4 \mu \mathrm{g}$ DNA per mg of $1.0 \mu \mathrm{M}$ gold particles). Prior to bombardment the petals were surface sterilised by immersion for $15 \mathrm{~min}$ in $10 \%$ $(\mathrm{v} / \mathrm{v})$ bleach containing a few drops of Tween-20, followed by three rinses in sterile water. The adaxial surface of the dorsal petals was bombarded. After bombardment, the plant materials were then cultured on $1 / 2 \mathrm{MS}$ medium under $20-50 \mu \mathrm{mol} \mathrm{m} \mathrm{m}^{-2} \mathrm{~s}^{-1}$ light from Osram $36 \mathrm{~W}$ grolux fluorescent tubes $(16 \mathrm{~h}$ photoperiod) at $25^{\circ} \mathrm{C}$. At least two flowers were used for each construct per experiment, and each experiment was repeated at least twice.

Potato cell suspension cultures for particle bombardment-mediated transformation were prepared by filtering $3 \mathrm{~mL}$ of culture onto sterile filter paper and subculturing them on a solid media in tubs for $48 \mathrm{~h}$ prior to transformation. The biolistic parameters were the same as those used for the antirrhinum petals with exception that they were only bombarded once. A steri-

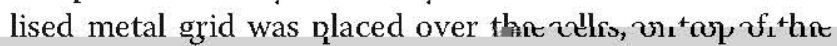
culture tub, to prevent displacement of the cells from the helium in-flow.

\section{Arabidopsis transformation}

The floral spray method developed by Clough and Bent [31] was used for the transformation of arabidopsis. Seeds were harvested from the Agrobacterium-inoculated plants and transgenics selected by spraying germinating seedlings with glufosinate herbicide (Basta, Bayer Crop Sciences). Positive transformants were grown through to production of $\mathrm{T} 2$ seeds following selfpollination.

\section{L-DOPA feeding}

Infiltration of antirrhinum petals with L-DOPA was carried out $24 \mathrm{~h}$ after biolistic transformation. The petals were placed in $10 \mathrm{mM}$ L-DOPA solution and a brief vacuum (30-60 s) applied, until the solution boiled vigorously and the petals became translucent. Following infiltration excess solution was blotted off and the petals incubated on $1 / 2$ MS medium under the same conditions as post-bombardment. Control petals were handled in the same manner but with sterile water substituted for L-DOPA. Petals were observed for betalain production after a further $24 \mathrm{~h}$. Transformed potato cell cultures were fed with $10 \mathrm{mM}$ L-DOPA solution by dispensing $1 \mathrm{~mL}$ of the solution on to the filter disks supporting the cells. The L-DOPA solution was applied to half of the samples immediately after transformation. 
Germinated arabidopsis seedlings were fed with 10 mM L-DOPA by transfer of the filter paper containing the seedlings on to a plate containing L-DOPA solution. Seedlings were observed for betalain pigment production after a further $12 \mathrm{~h}$. For feeding of inflorescences, six inflorescences were collected from 35S: AmDOD line 6 and cut to $1 \mathrm{~cm}$ long. Three inflorescences were immersed in $10 \mathrm{mM}$ L-DOPA solution and the other three immersed in water, and both sets were left for 2 min. The inflorescences were then placed into $1.5 \mathrm{ml}$ Eppendorf tubes with either $0.5 \mathrm{ml}$ of $10 \mathrm{mM} \mathrm{L-DOPA}$ solution or water, and the tubes left open on the bench for $24 \mathrm{~h}$.

\section{PCR analysis}

RT- PCR analysis for $D O D$ transgene expression in $35 S$ : $A m D O D$ arabidopsis plants used total RNA extracted from leaf tissue following the RNeasy protocol of the Qiagen RNeasy mini kit. Five rosette leaves were sampled from each of six individual T2 lines, as well as a non-transgenic wild type line. Following DNAse I treatment $500 \mathrm{ng}$ of RNA from each sample was used to generate cDNA using the Roche Transcriptor First Strand cDNA Synthesis Kit. PCR used Taq polymerase (Roche, New Zealand) and the following primers: DODF1 5'-CATACTACCATGTCCACCAAG-3', DODF 2 5'-AGCACTGCTTCTATATCGTC-3', Act2S 5'-TCC CTCAGCACATTCCAGCAGAT-3', Act2AS 5'-AACGATT CCTGGACCTGCCTCATC-3'. The Actin primers correspond to the arabidopsis Actin2 gene (AT3G18780). The thermocycling conditions were $94^{\circ} \mathrm{C}$ for $2 \mathrm{~min}$ and 25 cycles of $94^{\circ} \mathrm{C}$ for $20 \mathrm{~s}, 55^{\circ} \mathrm{C}$ for $30 \mathrm{~s}$ and $72^{\circ} \mathrm{C}$ for $30 \mathrm{~s}$. The PCR products were separated on a $1 \%(\mathrm{w} / \mathrm{v})$ TBE agarose gel containing ethidium bromide and visualised using UV-illumination. PCR products were also cloned and sequenced to confirm that the target PCR product was being amplified.

\section{HPLC analysis}

For arabidopsis analysis whole seedlings were extracted. For antirrhinum analysis, to ensure that similar petal regions were being compared, the ridge region of the biolistically transformed petals was excised away from the remainder of the petals for chemical analysis. The ridge is a distinct raised area that divides the lobes and throat of the dorsal petals. Ridge tissue from between three and six petals was pooled to give total sample fresh weights of 60 to $300 \mathrm{mg}$. Absorbance at $470 \mathrm{~nm}$ and $538 \mathrm{~nm}$ was used for detection of betaxanthins or betacyanins, respectively. Beetroot $(20 \mathrm{mg}$ freeze dried sample of red root) was used as a standard source of betalains, and strong peaks with the expected retention times and spectra data were observed.
Each sample was extracted three times in $1 \mathrm{ml}$ of $80 \%$ (v/v) methanol containing $50 \mathrm{mM}$ sodium ascorbate, as described in Schliemann et al. [14], with one overnight extraction at $4{ }^{\circ} \mathrm{C}$. The samples were centrifuged for 4 min at $10,000 \mathrm{rpm}$, the supernatant removed and the pellet re-extracted in the next $1 \mathrm{ml}$ of $80 \%$ methanol with ascorbate. The supernatants once removed, were combined to give the crude extract. The extract was dried in vacuo on a Savant SC210 Speedvac to near dryness, then freeze dried overnight to complete dryness. Extracts were resuspended in MilliQ water and made up to a final volume of $500 \mu \mathrm{l}$. The extracts were syringe filtered through a $0.45 \mathrm{um}$ nylon filter as per Svenson $e t$ al. [26] and the pigments analysed by high performance liquid chromatography (HPLC). The analysis of the antirrhinum samples used a Dionex 3000 Ultimate solvent delivery system with a Phenomenex Luna $(5 \mu \mathrm{m}, 150 \times$ $4.6 \mathrm{~mm}$ ) RP-18 endcapped column (column temperature $30^{\circ} \mathrm{C}$ ) and a Dionex 3000 Diode Array Detector (DAD). Elution $\left(1 \mathrm{ml} \mathrm{min}^{-1}\right)$ was performed using a solvent system comprising solvent A [1.0\% formic acid in water] and solvent $\mathrm{B}$ [ $80 \%$ acetonitrile in water] and a linear gradient starting with $100 \% \mathrm{~A}$, decreasing to $80 \% \mathrm{~A}$ at 62 mins, and then a linear gradient to $100 \% \mathrm{~B}$ at 67 mins, remaining at $100 \% \mathrm{~B}$ for a further $3 \mathrm{~min}$, then returning to initial conditions. Betaxanthins were detected at $470 \mathrm{~nm}$ and betacyanins at $538 \mathrm{~nm}$ [25]. The analysis of the arabidopsis samples used the solvent system of Schliemann et al. [14]. It was conducted on a Hewlett Packard HP 1100 with two Merck Chromolith analytical columns and a C-18 guard column.

\section{Additional material}

Additional file 1: PCR analysis for $D O D$ transgene expression in 35S: AmDOD arabidopsis plants. Total RNA was extracted from six lines of 355: AmDOD arabidopsis, as well as a non-transgenic wild type line, and analysed for DOD transcript levels using RT-PCR. PCR primers for an endogenous actin gene were used as a positive control for RNA/CDNA integrity. PCR products were separated on a $1 \%(\mathrm{~W} / \mathrm{v})$ agarose gel containing ethidium bromide and visualised using UV-illumination. (PPT $356 \mathrm{~kb}$ ).

\section{Acknowledgements \\ We thank lan King and Julie Ryan for care of plants, Steve Arathoon and John Harris for technical assistance, Willibald Schliemann for the A. muscario 4,5-DOPA dioxygenase cDNA vector, Andrew Gleave for pART7, Bart Janssen for $P M L B A R T$, Simon Coupe for pPN93, and the New Zealand Foundation for Research, Science, and Technology contract C02X0805 for supporting KES, SCD and KMD. NNH thanks The Todd Foundation, The Freemasons, NZ Federation of Graduate Women and the J. Skipworth Scholarship for their financial support. KEC thanks Victoria University for her PhD scholarship grant.}

\section{Author details}

'New Zealand Institute for Plant \& Food Research Limited, Private Bag 11600 , Palmerston North, New Zealand. ${ }^{2}$ School of Biological Sciences, 
University of Canterbury, Private Bag 4-800, Christchurch, New Zealand. Victoria University of Wellington, PO Box 600 , Wellington 6140, New Zealand. ${ }^{4}$ Commonwealth Scientific and Industrial Research Organization, Ecosystem Sciences, Urrbrea, South Australia 5064, Australia.

\section{Authors' contributions}

$\mathrm{NNH}$ conducted the AmDOD biolistic experiments in antirrhinum, produced and contributed to the analysis of the arabidopsis plants, and contributed to writing of the manuscript; JJ made pPN314 and conducted the biolistic experiments in potato; KMD conducted the PgDOD biolistic experiments in antirrhinum and wrote the main manuscript draft; DHL conducted the HPLC analysis of biolistically transformed material and contributed to the manuscript draft; PEJ contributed to supervision of $\mathrm{JJ}$ and $\mathrm{NNH}$ and project design; SCD assisted with cell culture experiments and supervision of Jj; KEC conducted $P C R$ analysis and inflorescence feeding for the arabidopsis plants KSG contributed to supervision of KEC. KES conceived and coordinated the study, supervised NNH and JJ, and contributed to analysis of the results and writing of the manuscript. All authors read and approved the final manuscript.

Received: 5 December 2011 Accepted: 12 March 2012

Published: 12 March 2012

\section{References}

1. Grotewold $\mathrm{E}$ : The genetics and biochemistry of floral pigments. Ann Rev Plant Biol 2006, 57:761-780.

2. Tanaka $Y$, Sasaki $N$, Ohmiya A: Biosynthesis of plant pigments: anthocyanins, betalains and carotenoids. Plant J 2008, 54:733-749.

3. Gould KS, Lister C: Flavonoid functions in plants. In Flavonoids: Chemistry, Biochemistry and Applications. Edited by: Andersen $\oslash \mathrm{M}$, Markham KR. CRC Press, Boca Raton; 2006:397-441.

4. Davies KM: An introduction to plant pigments in biology and commerce In Plant Piaments and their Manipulation. Edited by: Davies KM. Blackwell Publishing, Oxford; 2004:1-22

5. Strack D, Vogt T, Schliemann W: Recent advances in betalain research. Phytochemistry 2003, 62:247-269.

6. Zn̈d J-P, Christinet L: Betalains. In Plant Pigments and their Manipulation. Edited by: Davies KM. Oxford: Blackwell Publishing; 2004:185-213.

7. Brackington SF, Walker RH, Glover BJ, Soltis PS, Saltis DE: Complex pigment evolution in the Caryophyllales. New Phytol 2011, 190:854-864.

8. Mabry T: The betacyanins, a new class of red violet pigments, and their phylogenetic significance. New Yark: Roland Press; 1964

9. Gandia-Herrero F, García-Carmona F, Escribano J: Fluorescent pigments: new perspectives in betalain research and applications. Food Res int 2005, 38:879-884.

10. Gandía-Herrera F, Jiménez-Atiénzar M, Cabanes J, Escribana J, GarcíaCarmona F: Fluorescence detection of tyrosinase activity on dopaminebetaxanthin purified from Portulaca oleracea (Common Purslane) flowers. J Agric Food Chem 2009, 57:2523-2528

11. Christinet L, Burdet F, Zaiko M, Hinz U, Zn̈y JP: Characterization and functional identification of a novel plant 4,5-extradiol dioxygenase involved in betalain pigment biosynthesis in Portulaca grandifiora. Plant Physiol 2004, 134:265-274.

12. Gandía-Herrero F, Escribano J, García-Carmona F: Betaxanthins as substrates for tyrosinase An approach to the role of tyrosinase in the biosynthetic pathway of betalains. Plant Physiol 2005, 138:421-432.

13. Steiner U, Schliemann $W$, Böhm $H$, Strack D: Tyrosinase involved in betalain biosynthesis of higher plants. Planta 1999, 208:114-124.

14. Schliemann W, Kobayashi $N$, Strack D: The decisive step in betaxanthin biosynthesis is a spontaneous reaction. Plant Physiol 1999, 119:1217-1232

15. Sasaki $N$, Adachi $T$, Koda T, Ozeki Y: Detection of UDP-glucose cyclo-DOPA 5-O-glucosyltransferase activity in four o'clocks (Mirabilis jalapa L.). FEBS Lett 2004, 568:159-162

16. Sasaki N, Wada K, Koda T, Kasahara K, Adachi T: Isolation and characterization of cDNAs encoding an enzyme with glucosyltransferase activity for cyclo-DOPA from Four O'clocks and Feather Cockscombs. Plant Cell Physiol 2005, 46:666-670.

17. Mueller LA, Hinz U, Uze M, Sautter C, Zrÿd JP: Biochemical complementation of the betalain biosynthetic pathway in Portulaco grandiffora by a fungal 3,4-dihydroxyphenylalanine dioxygenase. Planta $1997,203: 260-263$
18. Mueller $L A, H i n z ~ U$, Zrÿd JP: The formation of betalamic acid and muscaflavin by recombinant DOPA-dioxygenase from Amanita. Phytochemistry 1997, 44:567-569.

19. Vogt $T$, Grimm R, Strack D: Cloning and expression of a cDNA encoding betanidin 5-O-glucosyltransferase, a betanidin- and flavonoid-specific enzyme with high homology to inducible glucosyltransferases from the Solanaceae. Plant / 1999, 19:509-519.

20. Vogt $T$, Zimmemann $E$, Grimm $R$, Meyer $M$, Strack D: Are the characteristics of betanidin glucosyltransferases from cell-suspension cultures of Dorotheanthus bellidiformis indicative of their phylogenetic relationship with flavonoid glucosyltransferases? Planta 1997, 203:349-361.

21. Shimada $S$, Inoue $Y T$, Sakuta M: Anthocyanidin synthase in non anthocyanin-producing Caryophyllales species. Plant J 2005, 58:950-959

22. Shimada S, Otsuki H, Sakuta M: Transcriptional control of anthocyanin biosynthetic genes in the Caryophyllales. J Exp Bot 2007, 58:957-967.

23. Shimada S, Takahashi $K$, Sato $Y$, Sakuta M: Dihydroflavonol 4-reductase cDNA from non-anthocyanin producing species in the Caryophyllales. Plant Cell Physiol 2004, 45:1290-1298.

24. Staffard HA: Anthocyanins and betalains: evolution of the mutually exclusive pathways. Plant Sci 1994, 101:91-98.

25. Kugler $F$, Stintzing FC, Carle R: Identification of betalains from petioles of differently colored Swiss Chand (Beta vuigaris L. ssp. cicla [L.] Alef. CV. Bright Lights) by High-Performance Liquid Chromatography-Electrospray Ionization Mass Spectrometry. J Agr Food Chem 2004, 52:2975-2981.

26. Svenson J, Smallfield BM, Joyce NI, Sansom CE, Perry NB: Betalains in red and yellow varieties of the andean tuber crop ulluco (Ullucus tuberosus) J Agr Food Chem 2008, 56:7730-7737.

27. Schwinn K, Venail J, Shang Y, Mackay S, Alm V, Butelli E, Oyama R, Bailey P, Davies K, Martin C: A small family of $M Y B$-regulatory genes controls floral pigmentation intensity and patterning in the genus Antirrhinum. Plant Cell 2006, 18:831-851.

28. Murashige T, Skoog F: A revised medium for rapid growth and bioassays with tobacco tissue cultures. Physiol Plantarum 1962, 15:743-747.

29. Gleave AP: A versatile binary vector system with a T-DNA organizationalstructure conducive to efficient integration of cloned DNA into the plan genome. Plant Mol Biol 1992, 20:1203-1207.

30. Shang $Y$, Schwinn KE, Hunter DA, Waugh TL, Bennett MJ, Pathirana NN, Brummell DA, Jameson PE, Davies KM: Methods for transient assay of gene function in floral tissues. Plant Methods 2007, 3:1.

31. Clough SJ, Bent AF: Floral dip: a simplified method for Agrobacteriummediated transformation of Arabidopsis thaliana. Plant J 1998, 16:735-743.

doi:10.1186/1471-2229-12-34

Cite this article as: Harris et al: Betalain production is possible in anthocyanin-producing plant species given the presence of DOPA dioxygenase and L-DOPA. BMC Plant Biology 2012 12:34.

\section{Submit your next manuscript to BioMed Central and take full advantage of:}

- Convenient online submission

- Thorough peer review

- No space constraints or color figure charges

- Immediate publication on acceptance

- Inclusion in PubMed, CAS, Scopus and Google Scholar

- Research which is freely available for redistribution

Submit your manuscript at

www. biomedcentral.com/submit

C) lorund Central 


\section{Appendix 4: Plasmid details}

\begin{tabular}{|l|c|c|c|}
\hline Plasmid name & Insert source & Construct information & Base vector \\
\hline pKEC1 & Beta vulgaris & $35 S_{\text {pro }}:$ CYP76AD1:OCS & pART7 \\
\hline pKEC2 & Beta vulgaris & $35 S_{\text {pro }}:$ CYP76AD1:OCS & pART27 \\
\hline
\end{tabular}

A.

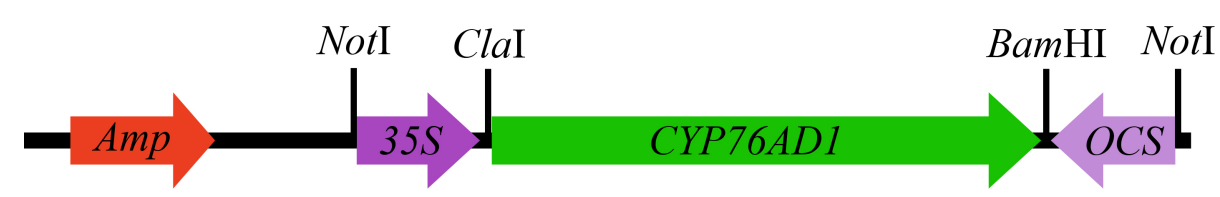

B.

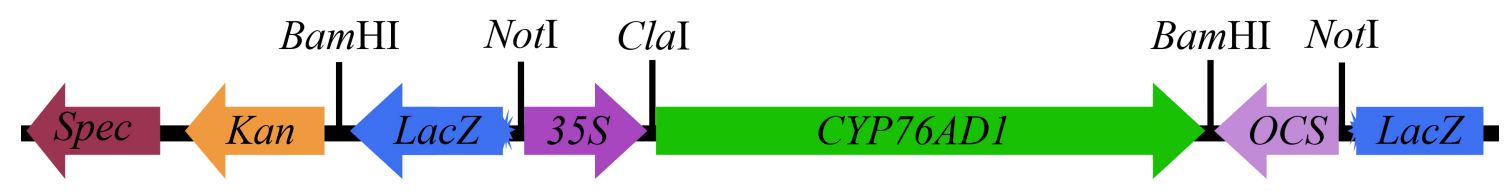

Appendix 4 Schematic illustration of CYP76AD1-containing plasmids: (A) pKEC1 and (B) pKEC2 (not drawn to scale). pKEC1 contains the ampicillin-resistance gene (Amp) with CYP76AD1 on a CaMV $35 S$ promoter and $A$. tumefaciens OCS terminator. pKEC2 contains the spectinomycin-resistance (Spec) and the kanamycin-resistance (Kan) genes with CYP76AD1 on a CaMV $35 S$ promoter and A. tumefaciens OCS terminator. The insertion point of CYP76AD1 in pKEC2 also interrupted the LacZ gene, enabling blue/white selection of this plasmid. Positions of restriction sites, ClaI, BamHI and NotI, used in the creation of these plasmids are marked on the diagrams. The total sizes of the plasmids are as follows: pKEC1 $=6534 \mathrm{bp}$ and $\mathrm{pKEC} 2=15338 \mathrm{bp}$. 


\section{Appendix 5: Gel electrophoresis images}
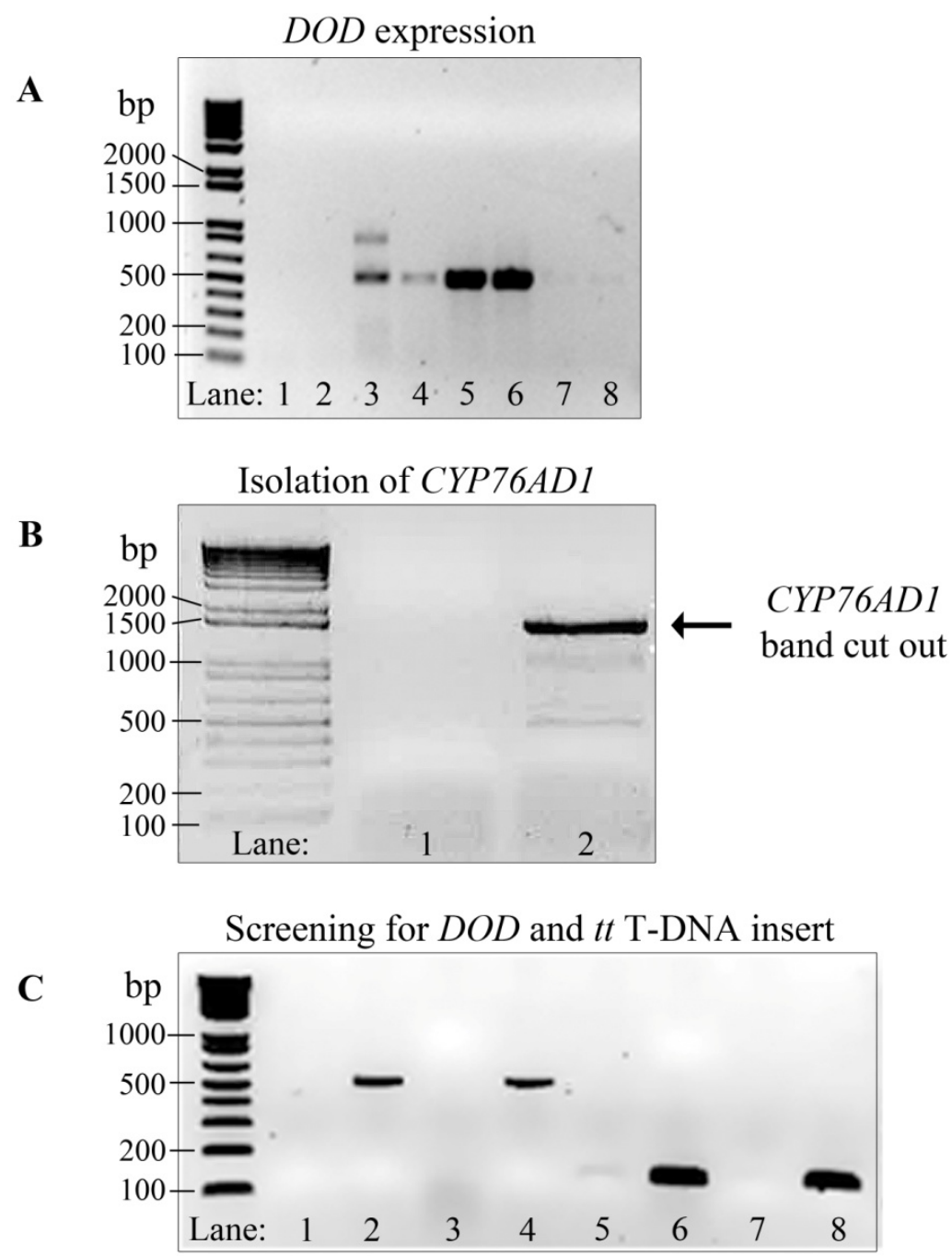

Salk screening for

D

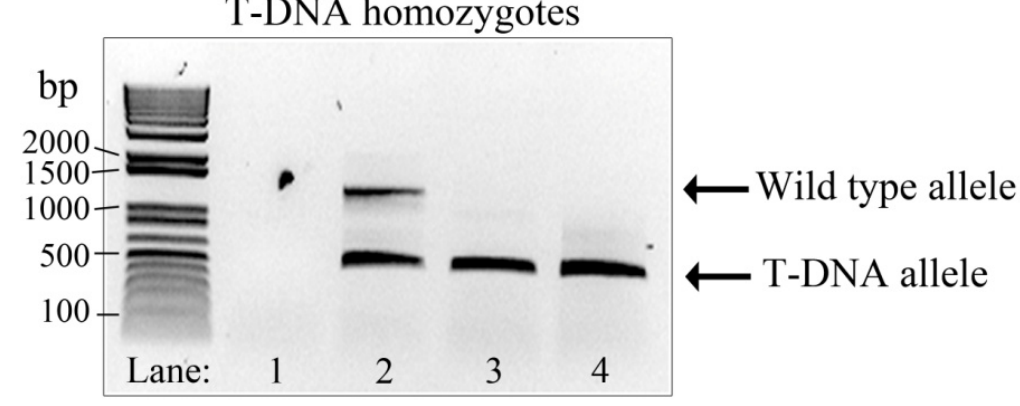

Appendix 5 PCR reactions on 1 - $2 \%$ (w/v) TBE gels. (A) DOD mRNA expression in different lines; Lanes: 1 - $\mathrm{H}_{2} \mathrm{O}, 2$ - negative biological control, 3 - 8 - DOD lines 1, 2, 6, 7, 8, 9. (B) Isolation of $C Y P 76 A D 1$ cDNA from Swiss chard; Lanes: $1-\mathrm{H}_{2} \mathrm{O}, 2-$ cDNA. (C) gDNA screening for DOD (lanes 1 - 4) and $t t$ T-DNA insert (lanes 5 - 8) in DOD x tt 12 plants; Lanes: $1 \& 5-\mathrm{H}_{2} \mathrm{O}, 2$ and 6 - positive control (DOD-6/tt12), 3 \& 7 negative control (tt12/DOD-6), $4 \& 8$ - DOD-6 x tt12. (D) gDNA Salk screening for T-DNA homozygotes; Lanes: $1-\mathrm{H}_{2} \mathrm{O}, 2$ - known heterozygote, 3 - known T-DNA homozygote, 4 - DOD-6 x tt12. All ladders are $1 \mathrm{~kb}$ Plus (Invitrogen). 


\section{Appendix 6: Phylogenetic alignment of $\mathrm{CHS}$}
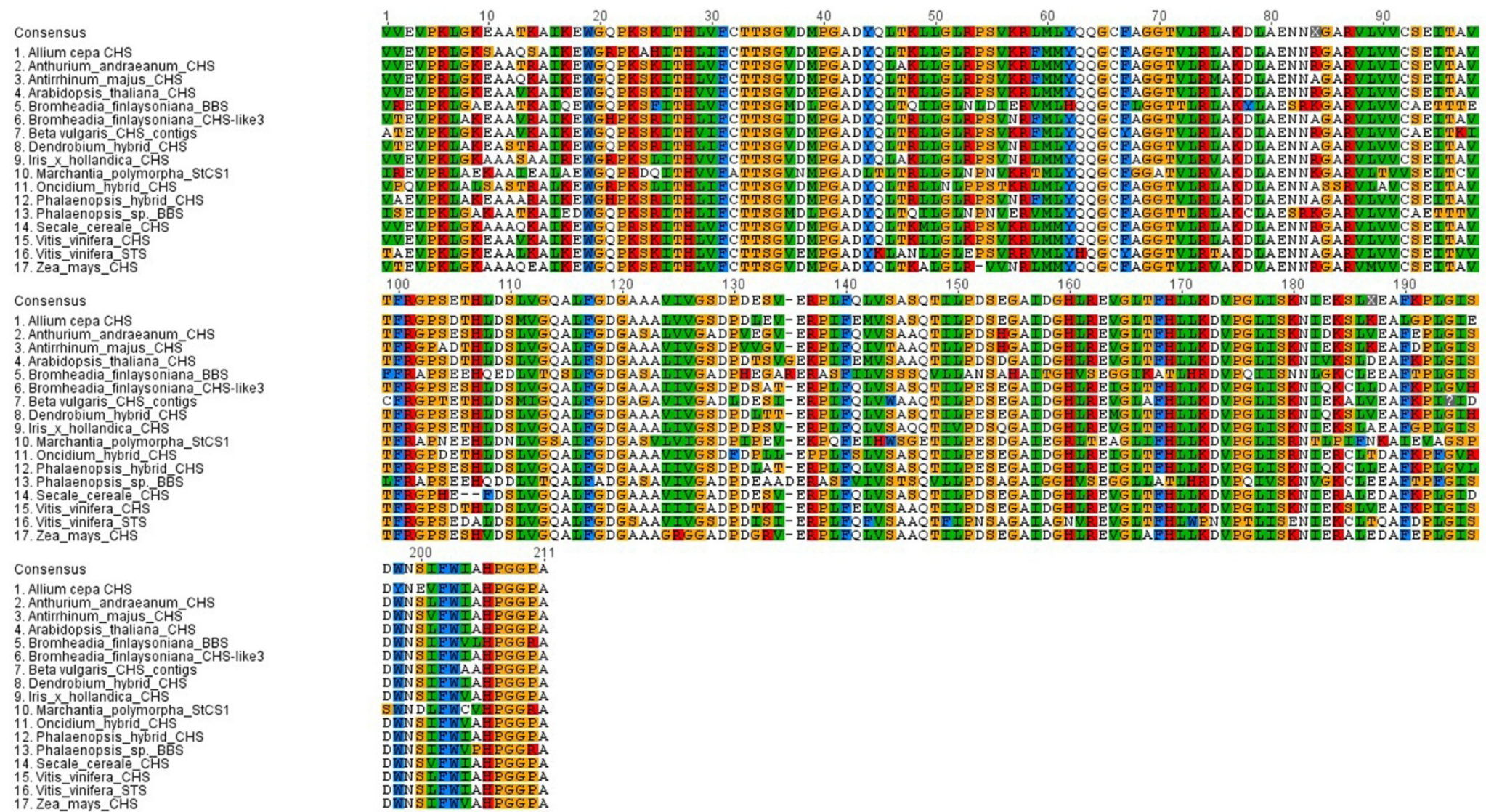

Appendix 6 Amino acid phylogenetic alignment (ClustalW, MEGA 5.2 (Tamura et al., 2011)) of the Beta vulgaris EST of CHS with equivalent regions other $C H S$ genes and structurally similar polyketide synthase genes. See Section 6.2.3.1 for species names and Genbank accession numbers. Colour-coding highlights sequence similarity. 


\section{Appendix 7: Phylogenetic alignment of DOD}

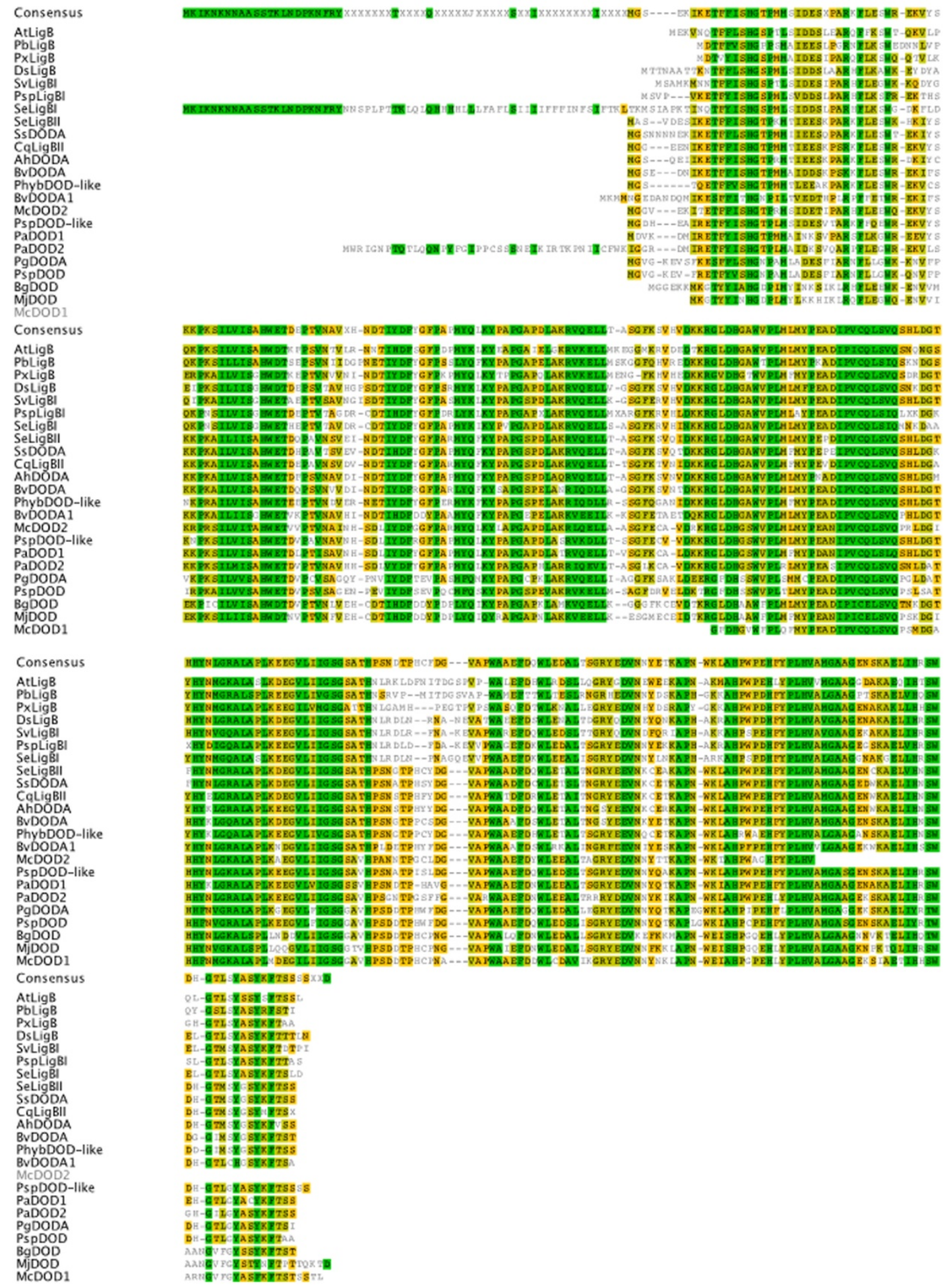

Appendix 7 Amino acid phylogenetic alignment (ClustalW, Geneious Pro 5.5.6, BioMatters Ltd.) of DODA and DODAl genes with other LigB homologues. See Section 6.2.3.2 for species names and Genbank accession numbers. Colour-coding highlights sequence similarity. Phylogenetic alignment supplied by Kathy Schwinn. 